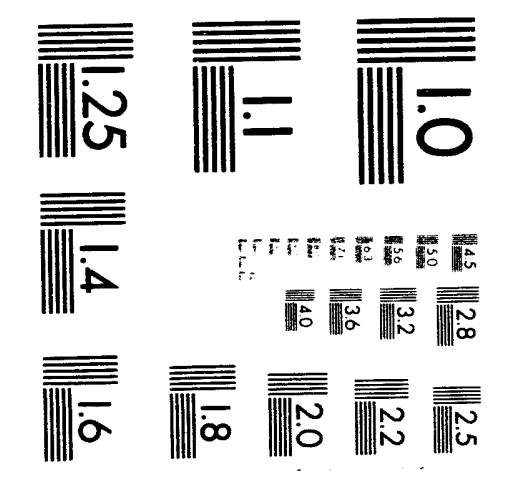



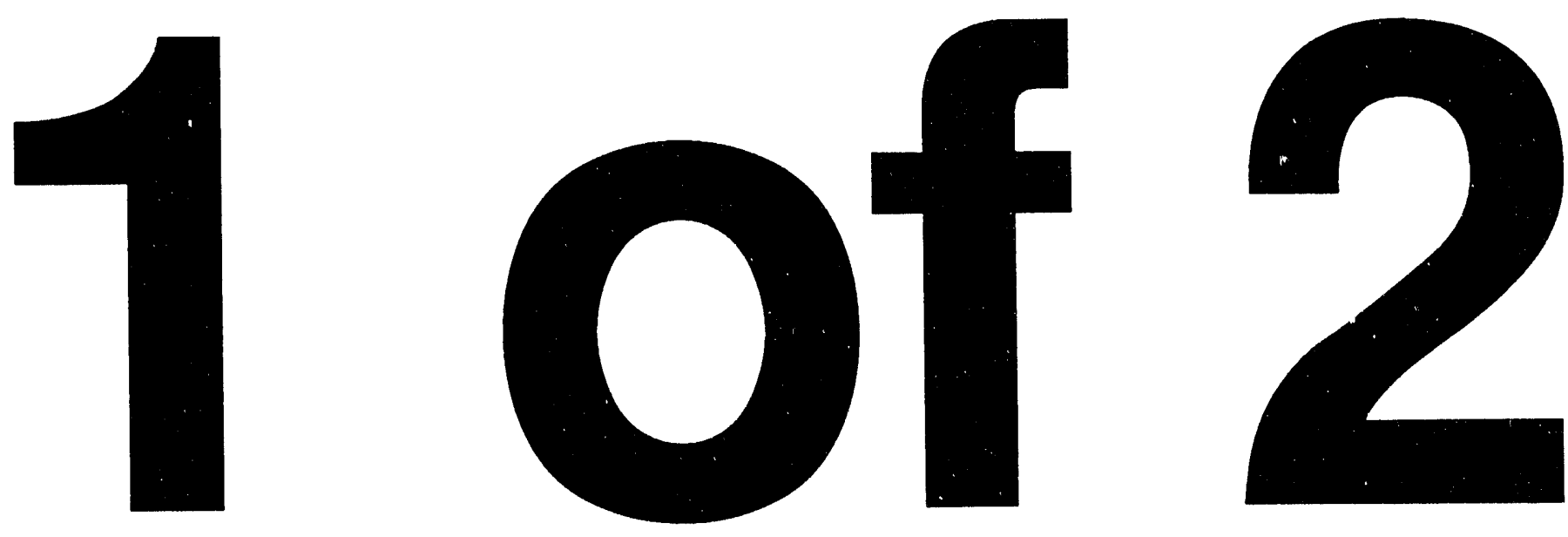


\section{LB Lawrence Berkeley Laboratory UNIVERSITY OF CALIFORNIA}

\section{CHEMICAL SCIENCES DIVISION}

\section{Application of Semiclassical Methods} to Reaction Rate Theory

R. Hernandez

(Ph.D. Thesis)

November 1993

FEO 221904

OSTI

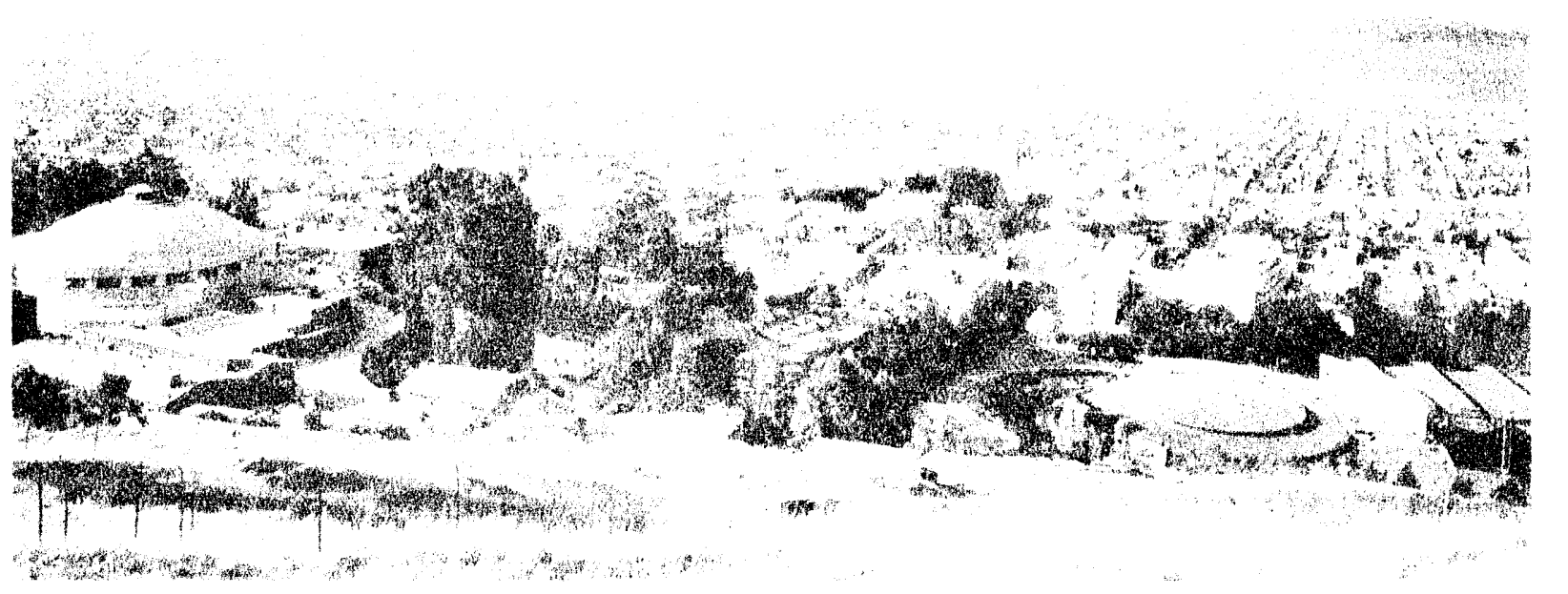

Prepared for the U.S. Department of Energy under Contract Number DE-AC03-76SF00098 


\section{DISCLAIMER}

This document was prepared as an account of work sponsored by the United States Government. Neither the United States Government nor any agency thereof, nor The Regents of the University of California, nor any of their employets, makes any warranty, express or implied, or assumes any legal liability or responsibility for the accuracy, completeness, or usefulness of any information, apparatus, product, or process disclosed, or represents that its use would not infringe privately owned rights. Reference herein to any specific commercial product, process, or service by its trade name, trademark, manufacturer, or otherwise, does not necessarily constitute or imply its endorsement, recommendation, or favoring by the United States Government or any agency thereof, or The Regents of the University of California. The views and opinions of authors expressed herein do not necessarily state or reflect those of the United States Government or any agency thereof or The Regents of the University of California and shall not be used for advertising or product endorsement purposes.

Lawrence Berkeley Laboratory is an equal opportunity employer. 
LBL-34925

\title{
Application of Semiclassical Methods \\ to Reaction Rate Theory
}

\author{
Rigoberto Hernandez \\ Ph.D. Thesis \\ Department of Chemistry \\ University of California
}

and

Chemical Sciences Division

Lawrence Berkeley Laboratory

University of California

Berkeley, California 94720

November 1993

This work was supported in part by the Director, Office of Energy Research, Office of Basic Energy Sciences,

Chemical Sciences Division of the U.S. Department of Energy under Contract No. DE-AC03-76SF00098, and in part by the National Science Foundation. 


\section{Application of Semiclassical Methods \\ to Reaction Rate Theory}

Copyright $(1991$

by Rigoberto Hernandez

The U.S. Department of Energy has to right to use this thesis for any purpose whatsoever including the right to reproduce all or any part thereof 


\author{
Abstract \\ Application of Semiclassical Methods \\ to Reaction Rate Theory \\ by \\ Rigoberto Hernandez \\ Doctor of Philosophy in Chemistry \\ University of California at Berkeley \\ Professor William H. Miller, Chair
}

This work is concerned with the development of approximate methods to describe relatively large chemical systems. This effort has been divided into two primary directions: First, we have extended and applied a semiclassical transition state theory (SCTST) originally proposed by Miller* to obtain microcanonical and canonical (thermal) rates for chemical reactions described by a nonseparable Hamiltonian, i.e. most reactions. Second, we have developed a method to describe the fluctuations of decay rates of individual energy states from the average RRKM rate in systems where the direct calculation of individual rates would be impossible. Combined with the semiclassical theory this latter effort has provided a direct comparison to the experimental results of Moore and coworkers. ${ }^{\dagger}$

In SCTST, the Hamiltonian is expanded about the barrier and the "good" action-angle variables are obtained perturbatively; a WKB analysis of the effectively one-dimensional reactive direction then provides the transmission probabilities. ${ }^{\ddagger}$ The advantages of this local approximate treatment are that it includes tunneling effects

\footnotetext{
*W. H. Miller, Faraday Discussions Chem. Soc, 62, 140 (1977).

'W. F. Polik, D. R. Guyer and C. B. Moore, J. Chem. Phys. 92, 3453 (1990).

tW. H. Miller, R. Hernandez, N. C. Handy, D. Jayatilaka and A. Willetts, Chem. Phys. Lett. 172, 62 (1990).
} 
and anharmonicity, and it systematically provides a multi-dimensional dividing surface in phase space. The SCTST thermal rate expression has been reformulated providing increased numerical efficiency (as compared to a naive Boltzmann average), an appealing link to conventional transition state theory (involving a "pre-reactive" partition function depending on the action of the reactive mode), and the ability to go beyond the perturbative approximation. ${ }^{\S}$

In addition, the distribution of unimolecular decay rates at threshold energies to dissociation has been modeled by describing the quasi-bound states as strongly-mixed. The possible existence of globally conserved symmetries - which would break this ansatz - is included by treating each symmetry block of the Hamiltonian separably and assuming the ansatz for each symmetry manifold. Use of SCTST to describe the reaction dynamics converts the model into a predictive theory depending only on the assumed dynamical symmetries. Comparison to the experimental decay rate distributions for $S_{0}$ formaldehyde dissociation" has revealed the presence of strong-mixing between the quasi-bound states and has further suggested that an electric field can break the $C_{s}$ symmetry of the dissociation."

${ }^{\S}$ R. Hernandez and W. H. Miller, Chem. Phys. Let. 214, 129 (1993).

IW. F. Polik, D. R. Guyer and C. B. Moore, J. Chem. Phys. 92, 3453 (1990).

"R. Hernandez, W. .H. Miller, C. B. Moore and W. F. Polik, J. Chem. Phys. 99, 950 (1993). 


\section{Contents}

Table of Contents $\quad$ v

List of Figures $\quad$ ix

List of Tables $\quad$ xi

Acknowledgments $\quad$ xiii

1 Statistics and Mode-Specificity 1

1.1 Semiclassical Transition State Theory (SCTST) . . . . . . . . . 3

1.1.1 Semiclassical Mechanics ............... 3

1.1.2 Transition State Theory ............... 6

$1.1 .3 \mathrm{SCTST} \ldots \ldots \ldots \ldots \ldots$

1.2 Random Matrix / Transition State Theory (RM/TST) . . . . . . . 9

1.2.1 The $\chi^{2}$ Distributions . . . . . . . . . . . . . . 10

1.2.2 Maximum-Likelihood Method . . . . . . . . . . . . 10

1.2.3 Random Matrix Theory . . . . . . . . . . . . . . . . . 12

1.2.4 The RM/TST Decay Rate Distributions . . . . . . . . . 16

1.3 Summary ........................ 17

2 SCTST - The "Standard" Model 18

2.1 Introduction . . . . . . . . . . . . . . . . 18

2.2 SCTST Transmission Probabilities . . . . . . . . . . . 20

2.2.1 Miller's Good Action-Angle Variables . . . . . . . . . . 20

2.2 .2 Methodology ................... 21

2.3 Perturbation Theory for the Activated Complex . . . . . . . . . . . . 23

2.3.1 General Theory . . . . . . . . . . . . . . . . . 23

2.3.2 Nondegenerate Vibrational Modes . . . . . . . . . . . 27

2.3.3 Degenerate Vibrational Modes . . . . . . . . . . . . . 28

2.3.4 Rotations . . . . . . . . . . . . . . . . 31

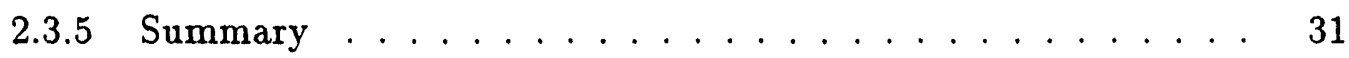

2.4 Illustrative Examples ..................... 33 
2.4 .1 Eckart Barrier . . . . . . . . . . . . . . . 34

2.4.2 Collinear $\mathrm{H}+\mathrm{H}_{2} \rightarrow \mathrm{H}_{2}+\mathrm{H} \ldots \ldots 36$

2.4.3 Full-dimensional $\mathrm{H}+\mathrm{H}_{2} \rightarrow \mathrm{H}_{2}+\mathrm{H} \ldots \ldots 37$

$2.4 .4 \mathrm{D}_{2} \mathrm{CO} \rightarrow \mathrm{D}_{2}+\mathrm{CO} \ldots \ldots 38$

2.5 Concluding Remarks . . . . . . . . . . . . . . . . . 42

3 SCTST - A New Perspective 43

3.1 Introduction . . . . . . . . . . . . . . . . 43

3.2 The "New" Semiclassical Rate Expression . . . . . . . . . . . . . 44

3.2.1 A Heuristic Derivation . . . . . . . . . . . . . . . . . 44

3.2.2 A Rigorous Derivation ................. 46

3.2.3 Construction of the Hamiltonian, $\mathcal{H}(\theta) \ldots \ldots$. . . . . . . 48

3.3 Illustrative Examples . . . . . . . . . . . . . . . . . 51

3.3.1 Eckart barrier . . . . . . . . . . . . . . . 51

3.3.2 Full-dimensional $\mathrm{H}+\mathrm{H}_{2} \rightarrow \mathrm{H}_{2}+\mathrm{H} \ldots \ldots 54$

3.3.3 $\mathrm{D}_{2} \mathrm{CO} \rightarrow \mathrm{D}_{2}+\mathrm{CO} \ldots \ldots \ldots 57$

3.4 Concluding Remarks .................... 59

4 SCTST - Perturbation Theory Revisited 60

4.1 Introduction . . . . . . . . . . . . . . . . . 60

4.2 CVPT for Vibrational Hamiltonians . . . . . . . . . . . . . . . 61

4.3 Mixed-Diagonalization . . . . . . . . . . . . . . . . 63

4.3 .1 Notation . . . . . . . . . . . . . . . . . 63

4.3.2 Obtaining the Generator ............... 65

4.3.3 Commutators Involving the Generating Function . . . . . . 65

4.3.4 Evaluation of $\left\langle n_{F}\left|\mathcal{H}_{0}^{(2)}\right| n_{F}\right\rangle \ldots \ldots 75$

4.3.5 The Second Order Hamiltonian Operator . . . . . . . . . . 76

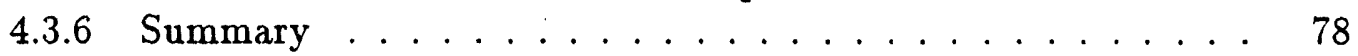

4.4 Application to Collinear $\mathrm{H}+\mathrm{H}_{2} \rightarrow \mathrm{H}_{2}+\mathrm{H} \ldots \ldots . \ldots 79$

4.5 Concluding Remarks ................... 81

5 RM/TST - Theory $\quad 83$

5.1 Introduction . . . . . . . . . . . . . . . 83

5.2 The RM/TST Model . . . . . . . . . . . . . . . 85

5.2.1 The Construction of the Model ............ 85

5.2.2 The Decay Rate Probability Distribution . . . . . . . . . . . 88

5.2.3 The Microcanonical Quantum Survival Probability . . . . . 89

5.2.4 The Effective Number of Channels: $\nu_{\text {eff }}$. . . . . . . . . . . 90

5.3 Model Calculations . . . . . . . . . . . . . . . . 95

5.3.1 The Porter-Thomas Limit . . . . . . . . . . . . . . . 95

5.3.2 Smooth Step Function Model ............... 96

5.3.3 Bi-Step Function Model . . . . . . . . . . . . . . 98 
5.4 A Predictive RM/TST Theory . . . . . . . . . . . . . . . . 101

5.4.1 The Eigenvalues of the Decay Rate Matrix . . . . . . . . . . 101

5.4.2 Relation Between $f$ and $\epsilon \ldots \ldots \ldots \ldots \ldots$

5.5 Symmetry Considerations . . . . . . . . . . . . . . . . . 104

5.5.1 The RM/TST Probability Distributions . . . . . . . 104

$5.5 .2 \nu_{\text {eff }}$ Using Moments . . . . . . . . . . . . 106

5.5.3 $\nu_{\text {eff }}$ Using the Maximum-Likelihood Method . . . . . . . . 107

5.5 .4 Summary . . . . . . . . . . . . . . . . . . . . . . 108

5.6 Concluding Remarks . . . . . . . . . . . . . . . . . . 108

6 RM/TST - Application to Formaldehyde 110

6.1 Introduction . . . . . . . . . . . . . . . . . . . . . 110

6.2 The Reduced Probability Distributions . . . . . . . . . . . . 111

$6.3 \mathrm{D}_{2} \mathrm{CO}$ Barrier Height . . . . . . . . . . . . . . . . . . . . 118

6.4 Comparison to Experiment . . . . . . . . . . . . . . . . . . . 122

6.4.1 The $J$-Resolved Distributions . . . . . . . . . . . . . . . . 123

6.4 .2 The Combined Distributions . . . . . . . . . . . . . . 126

6.4.3 The Effect of The Electric Field . . . . . . . . . . . . . . 127

6.5 Concluding Remarks . . . . . . . . . . . . . . . . 130

7 Discussion 132

$7.1 \quad$ SCTST . . . . . . . . . . . . . . . . . . . 132

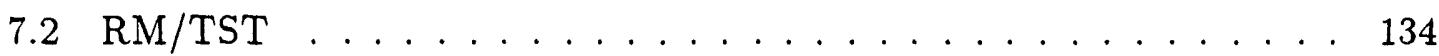

7.3 Final Observations . . . . . . . . . . . . . . 136

$\begin{array}{ll}\text { References } & 137\end{array}$ 


\section{List of Figures}

2.1 Eckart potential and its transmission probability. . . . . . . . 35

2.2 Cumulative reaction probability for collinear $\mathrm{H}+\mathrm{H}_{2}$. . . . . . . . 37

2.3 Cumulative reaction probability for full-dimensional $\mathrm{H}+\mathrm{H}_{2} \ldots \ldots$. . . 38

2.4 Boltzmann weighted cumulative reaction probability for $\mathrm{H}+\mathrm{H}_{2}$. . . . $\quad 39$

2.5 Transmission Probabilities for $\mathrm{D}_{2} \mathrm{CO}$ "fundamentals". . . . . . . . . 40

2.6 Transmission Probabilities for $\mathrm{D}_{2} \mathrm{CO}$ "overtones". . . . . . . . . 41

$3.1 \Gamma^{*}$ for Eckart potential. . . . . . . . . . . . . . . . 53

$3.2 \mathrm{H}+\mathrm{H}_{2}$ thermal rates. ...................... 56

$3.3 \mathrm{H}+\mathrm{H}_{2}$ pre-reactive partition function. . . . . . . . . 56

$3.4 \mathrm{D}_{2} \mathrm{CO}$ reactive partition function vs. temperature. . . . . . . . 57

$3.5 \mathrm{D}_{2} \mathrm{CO}$ pre-reactive partition function. . .......... 58

4.1 CRP for collinear $\mathrm{H}+\mathrm{H}_{2} \ldots \ldots \ldots$. . . . . . . . 80

5.1 Unimolecular decay rate vs. energy. . . . . . . . . . . . . . 84

5.2 Characteristic functional forms for $\left\{\gamma_{j}\right\}$ used in model distributions. . 96

5.3 Model distributions using smooth step form for $\left\{\gamma_{j}\right\}$ with $\nu_{\text {eff }}=6 . \quad$. 97

5.4 Model distributions using smooth step form for $\left\{\gamma_{j}\right\}$ with $\nu_{\text {eff }}=2$. . 98

5.5 Model distributions using bi-step form for $\left\{\gamma_{j}\right\}$ with $\nu_{\text {eff }}=6$. . . . 100

5.6 Model distributions using bi-step form for $\left\{\gamma_{j}\right\}$ with $\nu_{\text {eff }}=2$. . . . . 101

$6.1 J=0 \mathrm{RM} / \mathrm{TST}$ probability distributions. . . . . . . . . . . . 115

$6.2 J=2 \mathrm{RM} / \mathrm{TST}$ probability distributions. . . . . . . . . . . 116

$6.3 J=4 \mathrm{RM} / \mathrm{TST}$ probability distributions. . . . . . . . . . . . 117

$6.4 \chi^{2}$-distributions compared to experiment. . . . . . . . . . . . 118

$6.5 J$-resolved RM/TST distributions compared to experiment. . . . . . 124

6.6 Combined RM/TST distributions compared to experiment. . . . . . . 126

6.7 Combined RM/TST distributions compared to experiment distinguished by the electric field. . . . . . . . . . . . . . . . . 128

6.8 J-resolved RM/TST distributions compared to experiment distinguished by the electric field. . . . . . . . . . . . . . . . . . . 129 


\section{List of Tables}

2.1 The "spectroscopic constants" of the $\mathrm{H}+\mathrm{H}_{2}$ activated complex. . . . 33

$3.1 J=0$ thermal rate constants for $\mathrm{H}+\mathrm{H}_{2} \ldots \ldots \ldots 55$

6.1 The "spectroscopic constants" of the $\mathrm{D}_{2} \mathrm{CO}$ activated complex. . . . . 112

$6.2 \mathrm{RM} / \mathrm{TST}$ cumulative reaction probabilities vs. experiment. . . . . . 121

6.3 $A b$ initio and empirical bare barrier heights for the $\mathrm{D}_{2} \mathrm{CO}$ dissociation. 122

$6.4 \mathrm{RM} / \mathrm{TST}$ values of $\nu_{\text {eff }}$ vs. experiment. . . . . . . . . . . 125 


\section{Acknowledgments}

In the summer after my freshman year at Princeton, I had the opportunity to work in Prof. Kevin Lehmann's newly founded laboratory. I can not place a value on the many evenings in which Kevin would enter the laboratory and initiate discussion on whatever problem happened to be on his mind. It was this exposure to science which captured my interests and led me to pursue a doctoral degree in chemical physics.

The road to the completion of my degree has been long and there are several individuals who need be acknowledged. My doctoral advisor, Prof. William H. Miller, has always had his door open and it has been a pleasure to work with him. Dr. John Tully at AT\&T Bell Laboratories has gone beyond the call of duty in providing scientific and moral support. Many thanks also go to the members of the Miller group with whom I have interacted personally and scientifically: Dr. Scott M. Auerbach, Dr. YanTyng "Sherry" Chang, Dr. Daniel T. Colbert, Dr. Lionel F. X. Gaucher, J. Daniel Gezelter, Dr. Gerrit C. Grönenboom, Srihari Keshavamurthy, Prof. Claude J. Leforestier, Dr. Uwe Manthe, Dr. Camilla Minichino, Pamela Ohara, Dr. Beverly A. Ruf, Dr. Peter Saalfrank, Dr. Tamar Seideman, Prof. Moshe Shapiro, Bruce W. Spath, Dr. Gerhard Stock, Ward H. Thompson, Dr. Agathe Untch, Prof. David M. Wardlaw, Prof. Danny L. Yeager, and Prof. John Z. H. Zhang. It was an adventure, and I learned much from this group along the way. Of special note I should mention the untiring efforts of Cheryn Gliebe who made all of the administrative headaches go away. Profs. Bob Harris, David Chandler, Richard Saykallay and Robert Littlejohn have taught me a great deal through invaluable hours of lectures and private discussions.

It was the stimulating collaboration with Profs. Will Polik and C. Bradley Moore which resulted in the work presented in Chapters 5 and 6 . I also collaborated with Prof. Nicholas C. Handy, Dr. Andrew Willetts, Dr. Dylan Jayatilaka and Dr. Michael J. Cohen on the semiclassical transition state theory in Chapter 2. I am grateful to Dr. David W. Schwenke and Dr. David C. Chatfield for providing us with information regarding their calculations. ${ }^{1}$ Prof. J. N. L. Connor was kind enough to 
xiv

provide us with the exact $\mathrm{H}+\mathrm{H}_{2}$ collinear transmission probabilities on the LSTH surface. $^{2}$

But the road started a long time ago in Güinez, Havana, Cuba, and so I wish to thank my parents in a special way:

Gracias padres y madres míos por enseñar me que mi vos no es silente durante todos los momentos en cuales ustedes respondieron mis preguntas. Es posible que sin esa seguridad que ustedes me inculcaron, yo no pudiera haber terminado éste programa. Lo único que lamento es que nunca podre expresar en palabras mi gratitud por todo de lo que han hecho por mí.

I also wish to thank my friends who have supported me along the way: AA, JB, VC, CG, DG, ML, LR, RR, DS, DT, and TW.

Finally I would like to acknowledge the organizations which have provided the financial support necessary for this work and for the completion of my doctoral degree. In particular I wish to thank the National Science Foundation and the AT\&T Cooperative Research Fellowship Program (CRFP) for predoctoral fellowships. Much of the work has been supported by the Director, Office of Energy Research, Office of Basic Energy Sciences, Chemical Sciences Division of the U.S. Department of Energy under Contract No. DE-AC03-76SF00098. 


\section{Chapter 1}

\section{Statistics and Mode-Specificity}

In the treatment of unimolecular dissociation there has been a long standing debate with regards to the mode-specific - state-specific - chemistry of highly excited molecules. The two competing standard views have been the Slater theory ${ }^{3}$ and the RRKM (Rice, Ramsberger, Kassel and Marcus) theory. ${ }^{4-11}$ In the Slater theory, the vibrational modes are assumed to be harmonic, and the rate is the frequency that a combination of these vibrations achieves a critical extension. ${ }^{3,12}$ In the RRKM theory, one assumes that intramolecular vibrational redistribution (IVR) ${ }^{13}$ is fast compared to the dissociation rate and consequently all energy-allowed vibrational states are effectively involved in the dynamics. The rate is obtained as the ratio of the partition functions at the barrier - i.e. that of the activated complex - to that at the quasi-bound reactant well. The primary advantage of the RRKM theory is that it can readily include anharmonicity and this has been seen to be important in chemical reaction dynamics. ${ }^{14}$ Its disadvantage is that it treats the dynamics statistically, and can be in error if there is mode-specific behavior.

The natural questions to ask are whether or not mode-specific behavior occurs, and if so, what is the proper theory with which to treat it? The answer to the first question is that mode-specificity does occur, but it can be seen only in experiments with very detailed state preparation and/or observation. At the most rigorous level of detail the answer to the second question is "simple": write down the Schrödinger equation - within the Born-Openheimer approximation, for example - 
and solve the scattering problem; the rate is obtained from the $S$-Matrix by averaging over all the reactant and product quantum numbers which are not selected by the experimental apparatus and Boltzmann averaging over the energy. ${ }^{15}$ Even if one reduces the cost of the calculation by demanding that the dynamics is solved only to the extent that one directly obtains averaged quantities, the largest exact calculation that has been performed to date is a bimolecular reaction of a four atom system. ${ }^{16}$ What is needed, then, are approximate theories which readily provide dynamical quantities with reasonable accuracy for large systems.

In this work, we have developed and extended a semiclassical theory which provides averaged microcanonical and canonical rates in good agreement with exact theories. Although no explicit applications to truly large systems - e.g. 5 to 50 atom systems - are presented, the method is certainly applicable to these systems. The most detailed quantities that this semiclassical transition state theory (SCTST) provides are the transmission probabilities through the states of the activated complex. While these quantities are "mode-specific," they refer to ihe quantum states of the activated complex and not to the quantum states of the reactants or products probed in the experiment. In order to use this method to consider the mode-specificity of reactant states, one could use a vibrationally adiabatic approximation ${ }^{17-20}$ to connect the quantum states; however this approximation can be quite poor in high energy regimes where IVR between the quantum states is rapid.

An alternative approach is to treat the dynamics in the reactant region statistically while including the internal dynamics of the activated complex through the use of the SCTST. The random matrix / transition state theory (RM/TST) that we have developed builds upon this philosophy in order to describe the probability distributions of decay rates at energies near threshold. Comparison to the experimentally obtained state-specific decay rates $^{21}$ of formaldehyde suggests that this theory can indeed account for the fluctuations of the decay rates away from the average transition state rate. The fact that a theory which includes statistical and mode-specific components provides a good description of this process suggests that these concepts are not entirely incompatible. 


\subsection{Semiclassical Transition State Theory}

This section provides a brief review of the fundamental concepts of semiclassical mechanics and transition state theory which will be assumed in this work. In addition, a brief review of the semiclassical transition state theory to be developed in Chapters 2 to 4 is provided at the end. No attempt is made to make the citation list complete; the cited works are simply pointers to more thorough accounts.

\subsubsection{Semiclassical Mechanics}

Several excellent references exist describing the semiclassical treatment for bound state problems ${ }^{22-26}$ and that for systems with arbitrary boundary conditions which includes scattering events of relevance to the discussion of chemical reactions. ${ }^{27-32}$ In this short review, we restrict attention to scattering systems in which the dynamics is dominated by a single barrier and at energies where tunneling is important; the remainder of this work will almost exclusively be concerned with describing this regime.

\section{WKB}

By far the most common semiclassical method is the WKB method developed by Wentzel, Kramers and Brillouin. ${ }^{33-36}$ A rigorous derivation of the timeindependent form of this approximation begins by rewriting the wavefunction in the WKB form,

$$
\psi(\vec{r})=\exp [i S(\vec{r}) / \hbar]
$$

This expression diverges at classical turning points in the limit as $\hbar \rightarrow 0$, and so predicts an infinite density at these points whereas classically the density is zero at these points. ${ }^{28}$ The difficulty has emerged because Eq. (1.1) is nonanalytic in the region of $\hbar=0$, and Berry and Mount resolve this issue by recognizing that this limit is not quite the same as the classical limit, and hence define the $\hbar \rightarrow 0$ of Eq. (1.1) as the semiclassical limit. ${ }^{28}$ Substituting Eq. (1.1) into the time-independent Schrödinger 
equation leads to the following nonlinear equation in the complex quantity, $S(\vec{r})$ :

$$
-\frac{i \hbar}{2 m} \nabla^{2} S(\vec{r})+\frac{1}{2 m}(\nabla S(\vec{r}))^{2}+V(\vec{r})=E \text {. }
$$

where $E$ is the energy at which the stationary solution is obtained. At this stage $S(\vec{r})$ is expanded in powers of $\hbar$,

$$
S(\vec{r})=S_{0}(\vec{r})+\left(\frac{\hbar}{i}\right) S_{1}(\vec{r})+O\left(\hbar^{2}\right)
$$

where $S_{0}$ and $S_{1}$ are independent of $\hbar$. Using the above expansion one can easily see that the $O\left(\hbar^{0}\right)$ equation is the Hamilton-Jacobi equation ${ }^{37}$ for $S_{0}(\vec{r})$,

$$
\frac{1}{2 m}\left(\nabla S_{0}(\vec{r})\right)^{2}+V(\vec{r})=E
$$

and at $O\left(\hbar^{1}\right)$ one obtains the amplitude-transport equation:

$$
\frac{1}{2 m} \nabla^{2} S_{0}(\vec{r}, t)+\frac{1}{m}\left(\nabla S_{0}(\vec{r}, t) \cdot \nabla S_{1}(\vec{r}, t)\right)=0,
$$

which has the classical interpretation of a continuity equation in the density, $\left|S_{1}\right|^{2}$.

In one dimension, the independent WKB solutions of the Schrödinger equation are thus

$$
\Psi^{ \pm}(x)=[p(x)]^{-1 / 2} \mathrm{e}^{ \pm \frac{i}{\hbar} \int^{x} p\left(x^{\prime}\right) d x^{\prime}}
$$

These solutions diverge at caustics which, for this one-dimensional case in the $x$ representation, reduce to the classical turning points: $x_{\mathrm{tp}}$ such that $p\left(x_{\mathrm{tp}}\right)=0$. In order to write down the general solution for a one dimensional barrier problem, for example, one needs to obtain a connection formula between the coefficients of the solution on the left to those on the right of each such caustic. A particularly elegant approach to this is to switch representations, e.g. $x$ to $p$, transform the solutions on either side using the stationary phase approximation of the Fourrier transform $\left[=(-2 \pi i \hbar)^{-1 / 2} \int d x \mathrm{e}^{-i x p / \hbar}\right]$, and match these to the WKB solution in the $p$-representation. ${ }^{38}$ The result of such a calculation for the transmission probability is

$$
P(E)=\mathrm{e}^{-2 \theta}
$$


where the barrier penetration integral $\theta$ is defined by

$$
\theta \equiv \frac{1}{\hbar}\left|\int_{x_{1}}^{x_{r}} p\left(x^{\prime}\right) d x^{\prime}\right|
$$

where $x_{1}$ and $x_{\mathrm{r}}$ are the left and right caustics, respectively. This result is valid only for energies well below the barrier height in which the barrier is wide enough to support a WKB wave equation between the turning points.

\section{Uniform Approximations}

In order to extend the result of the WKB approximation, the method of comparison equations is used. ${ }^{39}$ One first constructs a model or comparison equation which contains the essential physics of the Schrödinger equation for the problem of interest, and which is also mathematically tractable. If one can then obtain a transformation which exactly or approximately transforms the correct Schrödinger equation into the the comparison equation, then the solution that is desired is obtained by using the corresponding transformation of the solutions of the comparison equation. This method is ideally suited for the treatment of tunneling because the solution of an inverted parabolic barrier is known, and hence one can compare the exact potential to the parabolic barrier in order to obtain the connection formula between the solutions across the barrier. ${ }^{39}$ The result is, (see, e.g., Ref. 28)

$$
P(E)=\left(1+e^{2 \theta}\right)^{-1}
$$

where $\theta$ is defined as in Eq. (1.8). Because this solution is correct for all energies, and not just below the barrier, it is often referred to as the uniform semiclassical approximation. In particular, an expansion of the uniform approximation in the tunneling regime results in the WKB result, Eq. (1.8), which suggests that this is the correct extension of the WKB theory.

Although the semiclassical treatment of one-dimensional tunneling is evidently well advanced, an open problem is to find equally good theories to describe multidimensional tunneling for nonseparable systems. (If the tunneling direction is separable from the rest, then the problem can be treated as one-dimensional with no 
loss of generality, and hence is well understood.) If the Hamiltonian is chaotic then the dynamics may be solved through the use of Gutzwiller's trace formula for the Green's function. ${ }^{40}$ The situation in which the Hamiltonian is not fully chaotic and is also non-separable is much less understood. One attempt at constructing a semiclassical theory for non-separable integrable Hamiltonians was proposed by Miller ${ }^{41}$ in which he takes advantage of the integrability condition which requires the existence of good action-angle variables, and obtains a solution by quantizing the good action variables. This method will be described more fully in Sec. 2.2.1, and it forms the basis for the semiclassical methodology developed in this work.

\subsubsection{Transition State Theory}

The foundations of transition state theory (TST) were developed as early as $1938 .{ }^{42}$ Remarkably, Wigner ${ }^{43}$ understood from the outset the three primary assumptions of transition state theory:

1. The electronic and nuclear motions are treated through the use of the BornOppenheimer approximation and the nuclear motion proceeds on a single adiabatic potential energy surface.

2. The nuclear motion is treated classically, or through the use of an approximate quantum mechanics.

3. No dynamical recrossings through the transition state (surface) are permitted.

The first of these assumptions is ubiquitous in chemical physics and will not be explored further in this work. (However, see Ref. 44 for non-adiabatic treatments of collision dynamics.) The remaining two assumptions are deeply connected and have been the subject of the advances that have occurred since the inception of transition state theory; several excellent reviews of these advances are available. ${ }^{45-49,15}$

In order to explore the stronger - classical - version of Wigner's second condition, we first recall the exact classical canonical - or thermal - rate from 
reactants $a$ to products $b$,

$$
k_{a \rightarrow b}^{c l}(T)=Q_{a}^{-1} h^{-1} \int d E \mathrm{e}^{-\beta E} N^{c l}(E),
$$

where $Q_{a}$ is the reactant partition function, and the microcanonical cumulative reaction probability is

$$
N^{c l}(E)=h^{1-\diamond} \int d \underset{\sim}{p} \int d q \delta[E-\mathcal{H}(\underset{\sim}{p}, \underset{\sim}{q})] \delta[f(q)] \times \frac{\partial f}{\partial \underset{\sim}{q}} \cdot \underset{\sim}{p} \mathcal{X}_{a \rightarrow b}(\underset{\sim}{p}, \underset{\sim}{q})
$$

where $f(q)$ is a field over the coordinate space and it takes the value zero on the dividing surface between reactants and products, i.e. the transition state surface, and $\mathcal{X}_{a \rightarrow b}(\underset{\sim}{p}, q)$ contains all of the dynamical information:

$$
\mathcal{X}_{a \rightarrow b}(\underset{\sim}{p}, \underset{\sim}{q}) \equiv \begin{cases}1, & \text { if } \underset{\sim}{q}(t \rightarrow-\infty) \text { is in reactant region } a \\ 0, & \text { otherwise. }\end{cases}
$$

To obtain the transition state theory result, one need only adopt Wigner's third condition, and the infinite-time dynamics needed to obtain Eq. (1.12) is reduced to the short-time - i.e., zero-time - result: ${ }^{45}$

$$
\mathcal{X}_{a \rightarrow b}(\underset{\sim}{p}, q) \approx h\left(-p_{s}\right)
$$

where $h$ is the Heaviside function which is one for positive arguments and zero otherwise. Thus TST will be exact if trajectories do not return to the transition state; Lafferty and Pechukas ${ }^{50}$ made this condition more precise for collinear reactions by presenting the geometric condition on the transition state surface which ensures that TST is exact below a given energy.

While the rate, Eq. (1.10), is invariant with respect to the choice of the transition state, the TST approximation is not. Here use is made of a variational principle which ensures that the optimal dividing surface is that for which the rate is minimized. ${ }^{51,52,48}$ The use of this principle is tantamount to minimization of the number of recrossings and thus improves the accuracy of Wigner's third assumption. The result is called a variational transition state theory (VTST), and can be further classified depending on whether the optimization of the thermal rate is performed using the same transition state surface for each energy - canonical VTST (CVT) - 
or whether transition states are optimized at each energy - microcanonical VTST $(\mu \mathrm{VT}) .{ }^{48}$ The latter is always smaller than the former and hence more accurate in accord with the variational principle, but it involves more computation time.

Pechukas and Pollak have further shown that TST is exact if there exists a single periodic orbit dividing surface (PODS). ${ }^{45,53-55}$ The PODS is defined as a periodic orbit which fills a dividing surface separating the reactant and product regions. If there are several such PODS, then the optimal choice of the variational transition state is still a PODS, and in this case TST is not exact. ${ }^{55}$

A natural extension of this TST or VTST is to extend $f$ to be a field over phase space - i.e. the space of coordinates and momenta. ${ }^{52}$ This generalization is necessary if the expression is to be invariant with respect to transformations of the phase space coordinates, and it is this generalized TST which will be referred to as TST throughout this work. (Since the fundamental objects in semiclassical mechanics live in phase space, this generalization is also a necessary prerequisite for the use of semiclassical quantization.)

The unresolved issues in TST primarily involve its application to quantum processes - i.e. the relaxation of Wigner's second assumption - such as reactions involving light atoms, e.g., hydrogen.$^{56,38,45,57}$ Given that a quantum particle cannot be confined to a single phase space point, it is not immediately obvious how to implement the zero-time assumption within a quantum framework. ${ }^{45,15}$ Even in the most ingenious quantum applications in which one uses optical potentials - or absorbing boundary conditions $(A B C)$ - in order to restrict the size of the potential energy surface needed for the rate calculation, one is still required to solve the dynamics exactly. ${ }^{15}$ Thus, in order to maintain the spirit of Wigner's third assumption, one needs to treat the mechanics at best semiclassically. ${ }^{38}$ In standard practice, the classical variational principle is used to optimize the transition state and quantum effects are included through the semiclassical treatment of an effective one-dimensional path projected from the multidimensional coordinate space. ${ }^{58,59}$ The optimal choice of this one-dimensional path is, unfortunately, not clear and many ad hoc choices of the path have been tried. ${ }^{48}$ 


\subsubsection{SCTST}

In this work, we will further develop a semiclassical transition state theory (SCTST) develped by Miller ${ }^{41}$ which incorporates the non-separability of the Hamiltonian in the region of the transition state. ${ }^{46}$ Following the spirit of Keck's generalized TST, ${ }^{52}$ the transition state is obtained not by variational optimization but by calculating perturbatively the approximate good action-angle variables of the local Hamiltonian. (Note that this surface need not be constructed explicitly in this formalism in order to calculate transmission probabilities or rates.) The SCTST avoids the indeterminacy of an effective path to be treated semiclassically but it introduces errors due to the perturbation theory.

This theory has further suggested a new semiclassical thermal rate expression which focuses attention on the calculation of a reduced dimensional Hamiltonian for the activated complex. The possibility that the vibrational modes may be coupled strongly and hence not be in a perturbative regime can be treated within this formalism by the construction of a mixed-diagonalization procedure to be developed in Chapter 4.

\subsection{Random Matrix / Transition State Theory}

Rather than focus on intrinsically non-RRKM behavior, here we are interested in the situation in which the chemical system agrees with the RRKM rate on the average, but that each state-selected decay rate does not. Stopping short of directly computing these values, the question which we will explore is how to describe the energy dependence of the fluctuations of these rates about the RRKM average. As in the previous section, the established theories are first described with a focus on the presentation of terminology and results which will be useful in the later discussion of the RM/TST theory in Chapters 5 and 6. A prelude of the RM/TST is also provided in Sec. 1.2.4. 


\subsubsection{The $\chi^{2}$ Distributions}

The established theory has used the $\chi^{2}$-family of distributions to describe the experimentally observed decay rate distributions. The members of this family as parametrized by $\nu$ are:

$$
P_{\nu}(\Gamma)=\frac{\nu}{2 G(\nu / 2)}\left(\frac{\nu \Gamma}{2 \Gamma}\right)^{\frac{\nu}{2}-1} \mathrm{e}^{-\frac{\nu \Gamma}{2 \Gamma}}
$$

where $G$ is the gamma function, and $\bar{\Gamma}$ is the average rate. ${ }^{60}$ In the literature, $\nu$ has alternately been called the number of degrees of freedom or the effective number of channels, depending on whether the emphasis is on statistics or physics, respectively. Here, we adopt the latter convention since $\nu$ is allowed to be non-integral and it will be seen that it can be interpreted as the number of channels effectively available to reaction. The moments of this distribution can be readily obtained:

$$
\begin{aligned}
\left\langle\Gamma^{n}\right\rangle & \equiv \int_{0}^{\infty} d \Gamma \Gamma^{n} P_{\nu}(\Gamma) \\
& =\frac{\bar{\Gamma} G\left(n+\frac{\nu}{2}\right)}{\left(\frac{\nu}{2}\right)^{n} G\left(\frac{\nu}{2}\right)} .
\end{aligned}
$$

From this result it is evident that $\nu$ can be expressed directly in terms of the moments:

$$
\nu=\frac{2 \bar{\Gamma}^{2}}{\left\langle\Gamma^{2}\right\rangle-\bar{\Gamma}^{2}} .
$$

and this expression has often been used to parametrize observed distributions. ${ }^{61,62}$ This procedure is an approximate way to choose an optimum member of the $\chi^{2}$ family, avoiding the direct computation in which one minimizes the $\chi^{2}$ error.

\subsubsection{Maximum-Likelihood Method}

In their study of nuclear energy level widths or decay rates, Porter and Thomas ${ }^{63}$ motivate the choice of the $\chi^{2}$ family of distributions by the use of a maximum-likelihood analysis. ${ }^{64}$ To obtain an optimum estimate, $P_{e}(\Gamma)$, of a distribution one maximizes the log likelihood,

$$
l\left(P_{e} \mid \mathrm{I}_{1}, \ldots, \Gamma_{N}\right) \equiv-\sum_{i=1}^{N} \ln P_{e}\left(\Gamma_{i}\right),
$$


where $\left\{\Gamma_{i}\right\}$ are a set of independent identically distributed observed rates, subject to smoothing constraints which guarantee the existence of a non-trivial maximum. ${ }^{65}$ In the analysis of Porter and Thomas, ${ }^{63}$ they consider the continuous case within the assumption that the frequency of a given observable, $\Gamma$, is correctly given by the estimate, $P_{e}(\Gamma)$, i.e., one maximizes the functional,

$$
S\left[P_{e}(\cdot)\right]=-\int d \Gamma P_{e}(\Gamma) \ln P_{e}(\Gamma),
$$

subject to constraints which depend on the observed data. The $\chi^{2}$ family of distributions results from the following choice of constraints:

$$
\begin{aligned}
\int d \Gamma \Gamma P_{e}\left(\Gamma_{i}\right) & =\bar{\Gamma}, \\
\int d \Gamma \ln (\Gamma) P_{e}\left(\Gamma_{i}\right) & =\langle\ln (\Gamma)\rangle .
\end{aligned}
$$

Since a $\chi^{2}$ distribution satisfies

$$
\int d \Gamma \Gamma P_{\nu}\left(\Gamma_{i}\right)=F\left(\frac{\nu}{2}\right),
$$

where

$$
F(z) \equiv G(z) \frac{\mathrm{d} G(z)}{\mathrm{d} z}-\ln z,
$$

it is apparent that the procedure to obtain $\nu$ for a given set of independent identically distributed observables is to solve the transcendental equation:

$$
F\left(\frac{\nu}{2}\right)=\frac{1}{N} \sum_{i=1}^{N} \ln \left(\Gamma_{i} / \bar{\Gamma}\right) .
$$

Note that if one were to require the second moment instead of $\langle\ln x\rangle$ as a constraint in a maximum-likelihood analysis, the resulting distribution would not be a $\chi^{2}$ distribution. Thus the use of the second moment to obtain the best fit $\chi^{2}$ distribution for the data is incompatible with the maximum-likelihood method.

A particularly useful result ${ }^{66}$ of the maximum likelihood analysis results from the observation that $\nu$ is related to the Lagrange multiplier for the constraint $(1.19 \mathrm{~b})$, and that this can be turned around to obtain an error estimate on $\nu$. The uncertainty in $\nu$ is: ${ }^{67,68}$

$$
\delta(\nu) \leq \frac{2 s}{\Delta[\ln (\Gamma / \bar{\Gamma})]},
$$


where

$$
\begin{aligned}
s^{2} & \equiv \sum_{j} f_{j}\left(\delta f_{j} / f_{j}\right)^{2} \\
\Delta^{2}[\ln (\Gamma / \bar{\Gamma})] & \left.\equiv\left\langle\left[\ln (\Gamma / \bar{\Gamma})-\left\langle\ln \left(\Gamma_{i} / \bar{\Gamma}\right)\right\rangle\right]^{2}\right\rangle \approx \frac{\mathrm{d}}{\mathrm{d} z}\left[G(z) \frac{\mathrm{d} G(z)}{\mathrm{d} z}\right]\right|_{z=\nu / 2},
\end{aligned}
$$

$f_{j}$ is the frequency of a given discretized observable $j$, and $\delta f_{j}$ is the difference between $f_{j}$ and the probability of seeing that observable given a $\chi^{2}$ distribution with $\nu$ effective channels. The principal disadvantage of this formula is that in order to calculate $s$, one needs to use a density estimate to obtain $f_{j}$ from the independent identically distributed observables $\left\{\Gamma_{i}\right\}$, and so the result depends strongly on the parametrization of the density estimate. Although there exist many sophisticated density estimates, ${ }^{65}$ in practice ${ }^{68}$ one estimates the density by binning the data into a histogram with each $j$ in Eq. (1.23) referring to a given bin. Note that from the comments above the error estimate in Eq. (1.23) is only appropriate if one obtains $\nu$ using Eq. (1.22), and consequently the $\nu$ obtained from the moments, Eq. (1.15), does not have a prescribed error estimate.

The $\chi^{2}$ family of distributions have been used to model nuclear energy level statistics successfully by several authors ${ }^{63,69,70,61}$ and its application to atomic transitions dates back to $1962 .{ }^{71}$ In applications to chemical physics, Levine and coworkers ${ }^{72,68,73}$ have actively pursued the interpretation and analysis of reaction rates using a similar analysis. Because of parallels with the information theoretic entropy, Levine ${ }^{72,68}$ calls the functional in Eq. (1.18) an entropy, and uses the term maximum entropy instead of maximum likelihood. Different constraints on the maximization of the entropy functional provide different dynamical results, and with subsequent comparison to experimental observations, these have been used to obtain information regarding the symmetries in the observed dynamics. ${ }^{72,68}$

\subsubsection{Random Matrix Theory}

An alternative approach for obtaining a statistical description of highly excited decaying states was proposed by Wigner. ${ }^{74}$ Several excellent reviews ${ }^{75-78,61,79}$ of this random matrix theory exist and here we concentrate only on those results which 
are pertinent to the discussion of the decay rate distributions. The philosophy behind this approach was eloquently proposed by Dyson: ${ }^{75}$

[...] It is improbable that level assignments based on shell structure and collective or individual-particle quantum numbers can ever be pushed as far as the millionth level. It is therefore reasonable to inquire whether the highly excited states may be understood from the diametrically opposite point of view, assuming as a working hypothesis that all shell structure is washed out and that no quantum numbers other than spin and parity remain good. The result of such an inquiry will be a statistical theory. The statistical theory will not predict the detailed sequence of levels in any one nucleus, but it will describe the general appearance and the degree of irregularity of the level structure that is expected to occur in any nucleus which is too complicated to be understood in detail.

$[\ldots]$ What is here required is a new kind of statistical mechanics, in which we renounce exact knowledge not of the state of a system but of the nature of the system itself. We picture a complex nucleus as a "black box" in which a large number of particles are interacting according to unknown laws. The problem then is to define in a mathematically precise way an ensemble of systems in which all possible laws of interaction are equally probable.

A natural question to ask then is how one chooses the ensemble ${ }^{77}$ and this will be reviewed below. Of more immediate relevance for the application to chemical systems is the question of whether this statistical view of the nuclear energy levels is appropriate for molecular energy levels.

On first inspection, one might argue that, in principle, the molecular Hamiltonian is known, and consequently there is no need to represent it by some ad hoc ensemble of Hamiltonians. While this may be true, it is also the case that for reasonably large molecules the cost of computing all of the vibrational states at high energies is prohibitive. Moreover, in a standard Born-Oppenheimer treatment, the potential energy surface would be sufficiently inaccurate in this high energy regime that the computed energy levels would be incorrect even if one were to attempt such a calculation. Thus, the need for a statistical theory is as relevant to molecular problems as it is to nuclear problems. But what is the analogue of "the large number of interacting particles in the complex nucleus" in the molecular system? In practice this is interpreted as the set of zeroth order states which are determined by diagonalizing 
an approximate Hamiltonian whose exact nature is unimportant, as the statistical results should not depend on it. The coupling between these zeroth order states as determined by the true Hamiltonian provides the unknown interaction between the zeroth order states. Given that the results of this formalism for various spectral measures - e.g., the nearest neighbor distribution of the energy spacings, $P(s)$, or the variance of the number of levels in a given energy interval, $\Sigma^{2}(E)$ - have provided good agreement with observed results for various chemical systems, ${ }^{80-84,21}$ it does therefore seem that random matrix theory is an appropriate model to use to describe molecular systems in high energy regimes; the interpretation of this agreement with regards to quantum chaos and IVR is still very much an open question. ${ }^{85-89,79,80,90}$

\section{Ensembles}

The three standard ensembles used in Random Matrix Theory are the Gaussian orthogonal ensemble (GOE), Gaussian unitary ensemble (GUE), and Gaussian symplectic ensemble (GSE). ${ }^{74,75,61}$ The simplest classification of these ensembles was shown in Dyson's threefold way in which their algebra is related to real, complex and quaternion numbers, respectively. ${ }^{91}$ The GOE is the ensemble of $d$-dimensional orthogonal matrices and it necessarily includes all bound state finite dimensional Hamiltonians, i.e., their matrix representation in a given finite dimensional basis. The probability that a given Hamiltonian is a member of the GOE is the joint probability distribution of its independent matrix elements where the diagonal elements are Gaussian distributed with variance $\sqrt{2 / d}$, and the off-diagonal elements are Gaussian distributed with variance $\sqrt{1 / d}$. The GUE is an ensemble with complex unitary matrices and corresponds to a system with no time reversal; for example, it includes the effective Hamiltonians of metastable states whose imaginary part corresponds to decay. The GSE is the ensemble of $d$-dimensional symplectic matrices - i.e., those which commute with the symplectic matrix,

$$
\mathbf{J}=\left(\begin{array}{cc}
\mathbf{0} & \mathbf{I} \\
-\mathbf{I} & \mathbf{0}
\end{array}\right),
$$

where $\mathbf{I}$ is the $d / 2$-dimensional identity matrix - and physically corresponds to systems which have time-reversal but not rotation invariance, e.g., relativistic Hamil- 
tonians. In the present work, only quasibound real orthogonal Hamiltonians will be treated statistically, and as such the GOE is the one which will receive the most attention. (Note, though, that the full Hamiltonian for the decay process is complex and if it were to be treated entirely statistically, the GUE would be a more appropriate choice for the ensemble.)

The primary advantage of the standard ensembles is their mathematical tractability; their disadvantage is that the symmetry requirements are minimal and consequently there is little specification of the particular symmetries of a chosen system. ${ }^{92}$ It is surprising that given this lack of specification of the system in the standard ensembles, it nonetheless provides spectral measures in excellent agreement with those of a large majority of physical systems. ${ }^{61}$ Motivated by the goal to attain more physical insight from spectral measures, however, much of the work in this field has been focused on the development of new ensembles whose restrictions correspond to further symmetries of a system; the review in Ref. 61 and several references therein describe various choices of these ensembles. In particular, Leitner and coworkers ${ }^{93,94}$ have defined several ensembles in which coupling between different states is treated at different orders and so can account for partial breakdown of dynamical quantum numbers. While the RM/TST to be described in Chapter 5 only accounts for the possibility that quantum numbers are either entirely conserved or strongly mixed, the possibility of relaxing this assumption in the calculation of decay rate distributions will be discussed in Chapter 7 , within the context of Leitner's ensembles.

\section{Decay Rate Distributions}

The decay rate from a given quasi-bound state $i$ to a final continuum state $f$ is

$$
\Gamma=|\langle i|T| f\rangle|^{2}
$$

where $T$ is the transition operator representing the dynamics of the decay. If $T$ is a projection operator onto a single quantum state - i.e., the decay channel, ${ }^{95}$ - then the probability distribution of $\Gamma$ after averaging over $i$ is a $\chi^{2}$-distribution with 1,2 or 4 degrees of freedom depending on whether the statistics of the quasi-bound states 
$i$ are treated as those of the GOE, GUE or GSE, respectively. ${ }^{77,61}$ Arbitrary integer values of $\nu$ can be obtained using the GOE to describe the quasi-bound states, but now considering a decay process in which several channels $n$ are equally open. How exactly one considers deviations from the $\chi^{2}$-distributions through the use of a nonstatistical and a priori treatment of the transition strengths of these decay channels is the subject of the RM/TST distributions to be developed in this work.

\subsubsection{The RM/TST Decay Rate Distributions}

The question which was suggested by the results of Polik et al. ${ }^{96,21}$ is: Can one find a theoretical basis for describing decay rate distributions which differ from the $\chi^{2}$ distributions but whose underlying spectral energies are in agreement with the GOE? In a study of decay rate distributions for systems with two coupled surfaces, Cederbaum and coworkers ${ }^{97}$ use a less simplistic model of the decay than that suggested by Eq. (1.25), but the energy spectrum also differs from the GOE. Thus Polik et al. ${ }^{98,99}$ suggest a model in which the quasi-bound (or metastable) states obey GOE statistics, the decay rate for each of these metastable states is calculated within a first order treatment (appropriate when the widths are resolved), and the probability distribution obtains by averaging over the rate of each metastable state weighted by its GOE density. As will be shown in Chapter 5, this model includes the $\chi^{2}$ distributions as a limiting case but, in general, can be quite different from the $\chi^{2}$ family.

Encouraged by the fact that this model can be fit to the experimental histograms with excellent agreement, ${ }^{100}$ in Chapter 5 we proceed to use a semiclassical model to describe the decay through the barrier in order to obtain a predictive theory for the distributions. Furthermore this model readily allows for the possibility that there may exist dynamical symmetries which are obeyed throughout the dissociation. In order to satisfy the GOE requirement that the states in the ensemble are labeled by all known symmetries, one simply separates the Hamiltonian into its block diagonal form (labeled by the appropriate symmetry quantum numbers) and uses the single symmetry analysis on each block. The resulting distributions are no longer necessarily unimodal and this further extends the family of allowed distributions. 
Chapter 6 presents an analysis of the $\mathrm{D}_{2} \mathrm{CO}$ dissociation (decay) rate distribution observed by Polik et al. ${ }^{96,21}$ including a treatment of angular momentum. Use of semiclassical transition state theory provides an estimate of the barrier height to dissociation. The only adjustable "parameter" in the RM/TST distributions is the choice of dynamical symmetries which are assumed to be conserved throughout the dissociation; comparison of the theory, given each of these choices, to the experimental results then determines the dynamical symmetries of the physical system.

\subsection{Summary}

The semiclassical transition state theory (SCTST) is developed in this work affords the possibility to include quantum effects in large systems, albeit only approximately. In particular, the SCTST plays a crucial role in the use of RM/TST theory to determine a priori probability distributions of the $\mathrm{D}_{2} \mathrm{CO}$ decay rates at energies near the dissociation threshold. The remarkable agreement between the RM/TST distributions and the experimental results suggests that in at least some molecules, state-specific behavior can be described within quasi-statistical theories. 


\section{Chapter 2}

\section{Semiclassical Transition State Theory - The "Standard" Model}

\subsection{Introduction}

The thermal rate constant for a chemical reaction can be conveniently expressed as a Boltzmann average (or Laplace transform) of the cumulative reaction probability (CRP) $N(E),{ }^{101,102,38}$

$$
k(T)=\left(2 \pi \hbar Q_{r}\right)^{-1} \int_{\mathcal{E}_{0}}^{\infty} d E \mathrm{e}^{-\beta E} N(E),
$$

where $Q_{r}$ is the reactant partition function (per unit volume), $\mathcal{E}_{0}$ is the quantum mechanical reaction threshold below which $N(E)$ is zero, and $\beta \equiv(k T)^{-1}$ is proportional to the inverse temperature as usually defined. The microcanonical rate, typically of most interest for unimolecular reactions, is also given in terms of the CRP as

$$
k(E)=\left[2 \pi \hbar \rho_{r}(E)\right]^{-1} N(E),
$$

where $\rho_{r}$ is the reactant density of states (per unit energy).

Considerable effort has been focused recently on developing numerically efficient methods to evaluate the CRP exactly, ${ }^{15}$ but at a much simpler level it can be evaluated through the implementation of a semiclassical transition state theory 
(SCTST) approximation ${ }^{41}$ for the CRP. ${ }^{103,104}$ Because SCTST is much easier to implement than more rigorous numerical approaches, it can be more readily applied to complex reactions. Though this SCTST clearly contains the basic transition state approximation of "direct dynamics", it nevertheless includes the effect of non-separable couplings between all degrees of freedom - including the reaction coordinate - in the transition state region. We also note that SCTST is similar to the most general form of variational TST ${ }^{52}$ in that it corresponds ${ }^{41}$ to computing flux through a dividing surface in the full phase space of the system; the commonly used version ${ }^{48}$ of variational TST only considers dividing surfaces in coordinate space.

The use of $a b$ initio methods ${ }^{105,106}$ to obtain up to quartic derivatives of the potential energy surface has recently enabled the use of the semiclassical formulae (to be presented in Sec. 2.2) to include anharmonicity in the calculation of the CRP $\mathrm{P}^{103}$ and thermal rates ${ }^{104}$ for systems of chemical interest. In analogy to the assignment of rovibrational energy levels performed with canonical Van Vleck perturbation theory (CVPT), ${ }^{107-109}$ the (not-necessarily) separable Hamiltonian is obtained by expanding the Hamiltonian in a Taylor expansion about the saddle point (or transition state) and performing the perturbation to an order consistent with the expansion. For example, as will be shown in Sec. 2.3, to obtain the second order vibrational Hamiltonian one need only include the cubic and a limited set of the quartic terms in the anharmonic part of the original expansion. The formulae presented in these sections also explicitly include angular momentum $J$. This affords the possibility of computing experimental thermal rate constants which is particularly relevant in the application to probability distributions which is the subject of Chapters 5 and 6 .

Results using this method with $J=0$ are presented in Sec. 2.4. In particular, the CRP for the reaction

$$
\mathrm{H}+\mathrm{H}_{2} \rightarrow \mathrm{H}_{2}+\mathrm{H},
$$

is obtained for various cases of dimensionality of the reactive space: (a) a onedimensional (Eckart) barrier; $(b)$ a collinear reaction; and $(c)$ in full dimensionality. The transmission probabilities for the $\mathrm{D}_{2} \mathrm{CO}$ dissociation are also presented in this section. As manifested by this list of systems, the SCTST is clearly applicable both 
to unimolecular and bimolecular reactions.

\subsection{SCTST Transmission Probabilities}

\subsubsection{Miller's Good Action-Angle Variables}

The formally exact quantum mechanical expression for the cumulative reaction probability ${ }^{38}$ in Eq. (2.1) is

$$
N(E)=2 \pi \hbar \operatorname{tr}[\delta(E-\mathcal{H}) \delta(f) \dot{f} \mathcal{P}],
$$

where $f$ is an operator that defines the transition state surface - e.g. $\langle x|f| x\rangle<0$ for $x$ in the reactant region - and $\mathcal{P}$ is a projection operator which projects onto all states which have evolved from reactants in the intinite past. Mille ${ }^{41}$ has shown how this expression may be evaluated semiclassically to obtain a transition state CRP for non-separable Hamiltonians. This theory relies on the existence of good (global) action-angle variables for the Hamiltonian, and it is briefly described in this section.

Following Ref. 41, if the Hamiltonian is integrable, but not necessarily separable in the $F$ good actions, $\left\{n_{i}\right\}$, then the quantum system can be described by energy eigenstates, $|\underline{\sim}\rangle$, i.e.

$$
\mathcal{H}|\underline{n}\rangle=E(\underline{n})|\underline{\sim}\rangle \text {. }
$$

The CRP is then obtained by replacing the quantum wavefunctions with the corresponding semiclassical wavefunctions and performing the trace in Eq. (2.4). Since all but one of these integrals can be performed by the stationary phase approximation, ${ }^{101}$ it is natural to choose the last remaining integral to be over the angle variable corresponding to the reactive direction. The result is ${ }^{41}$

$$
N(E)=\int d n_{F} \sum_{n^{\ddagger}} \delta\left[E-E\left(\underline{n}^{\ddagger}, n_{F}\right)\right] \frac{\partial E\left(\underline{n}^{\ddagger}, n_{F}\right)}{\partial n_{F}}\left(1+\mathrm{e}^{2 \pi \operatorname{lm} n_{F}}\right)^{-1},
$$

where the action corresponding to the reaction coordinate, $n_{F}$, has been distinguished from the remaining actions, $n^{\ddagger}$. (The notation is suggested by the separable case in which $n_{\sim}^{\ddagger}$ are the good actions corresponding to motions perpendicular to the reaction coordinate.) 
After reversing the order of the integration and the summation in Eq. (2.6), the integral with respect to $n_{F}$ provides the standard result: ${ }^{101}$

$$
\begin{aligned}
N(E) & =\sum_{\mathbf{n}^{!}}\left[1+\mathrm{e}^{2 \pi \operatorname{Im} n_{F}\left(E, \underline{n}^{\natural}\right)}\right]^{-1} \\
& =\sum_{n^{!}} P_{n^{!}}(E)
\end{aligned}
$$

where $P_{n^{\ddagger}}\left[=\left\{1+\exp \left(2 \pi \operatorname{Im} n_{F}\right)\right\}^{-1}\right]$ is the transmission probability through a given state of the activated complex. Use of the Bohr-Sommerfeld quantization rule, suggests the association,

$$
n_{F}\left(E, n^{\ddagger}\right)+\frac{1}{2}=i \theta\left(E, n^{\ddagger}\right) / \pi,
$$

where $\theta$ can be viewed as the generalization of the one-dimensional barrier penetration integral. ${ }^{110}$ This allows the CRP to be cast in the suggestive form:

$$
N(E)=\sum_{n^{!}}\left[1+\mathrm{e}^{2 \theta\left(E, n^{t}\right)}\right]^{-1} .
$$

\subsubsection{Methodology}

The starting point for the extension of the semiclassical TST just described is to note that the classical Hamiltonian can, in general, be expressed in terms of a set of locally conserved ("good") actions associated with the transition state (i.e., saddle point) region of the potential energy surface. The first step in constructing the SCTST is thus to determine the classical Hamiltonian $\mathcal{H}^{\mathrm{cl}}(\underline{I}) \equiv \mathcal{H}^{\mathrm{cl}}\left(I_{1}, \ldots, I_{F}\right)$ in terms of the $F$ actions $\left\{I_{k}\right\}$ (where $F$ is the number of degrees of freedom.) The $(F-1)$ actions associated with the bound degrees of freedom are quantized in the usual semiclassical (Bohr-Sommerfeld) fashion, ${ }^{111}$

$$
I_{k}=\left(n_{k}+\frac{1}{2}\right) \hbar \text { for } k=1, \ldots, F-1,
$$

where $n^{\natural} \equiv\left\{n_{k}\right\}$ are the quantum numbers for states of the activated complex. The action $I_{F}$ - the one associated with the reaction coordinate - is pure imaginary and defines the generalized barrier penetration integral ${ }^{10} \theta$,

$$
\theta \equiv-i \pi I_{F} / \hbar \text {. }
$$


(We refer to $\theta$ as the generalized barrier penetration integral because it is given by the well-known integral

$$
\theta=\int_{\text {barrier }} d q \sqrt{2 m(V(q)-E)}
$$

for a one-dimensional barrier.) $\theta$ is determined as a function of total energy $E$ and the $(F-1)$ quantum numbers of the activated complex by energy conservation

$$
\mathcal{H}^{\mathrm{cl}}\left[I_{k}=\left(n_{k}+\frac{1}{2}\right) \hbar, I_{F}=i \hbar \theta / \pi\right]=E
$$

i.e., for $\underline{\sim}^{\ddagger}$ fixed, one must invert the $E-\theta$ relation defined by Eq. (2.13) to obtain $\theta\left(E, n^{\ddagger}\right)$. Since the dynamics is integrable in terms of the "good" actions, the transraission probability for state $\underline{\sim}^{\ddagger}$ and energy $E$ has the same form as in one dimension, i.e., $\left(1+e^{2 \theta}\right)^{-1}$, so the CRP is given by ${ }^{41}$

$$
N(E)=\sum_{\underline{n}^{t}}\left[1+\mathrm{e}^{2 \theta\left(E, n^{t}\right)}\right]^{-1} .
$$

Note that while in this discussion the semiclassical Hamiltonian has been constructed from the classical Hamiltonian, it is also possible to obtain it from the quantum Hamiltonian $\mathcal{H}^{\mathrm{qm}}(\underset{\sim}{n})$ if one is able to determine the latter in terms of a complete set of good quantum numbers $\underset{\sim}{n}$. [This connection has recently been made more explicit by the demonstration that the semiclassical energies of the activated complex correspond to the (Siegert) eigenvalues of the complex Hamiltonian with imposed outgoing wave boundary conditions. $\left.{ }^{112}\right]$ The generalized barrier penetration integral is defined in analogy with Eq. (2.11) as

$$
\theta \equiv-i \pi\left(n_{F}+\frac{1}{2}\right)
$$

where $n_{F}$ is the vibrational "quantum number" associated with the reactive mode. Because this mode is not bound, $\theta$ is again a continuous variable and may be obtained by inverting the Hamiltonian equation,

$$
\mathcal{H}^{\mathrm{qm}}\left[\stackrel{n}{\ddagger}^{\ddagger}, n_{F}=\left(i \theta / \pi-\frac{1}{2}\right)\right]=E .
$$

It may appear that this procedure is difficult to implement because of the difficulty associated with obtaining the quantum Hamiltonian $\mathcal{H}\left({\underset{\sim}{n}}^{\ddagger}, \theta\right)$. In fact, $\mathcal{H}\left(n_{\sim}^{\ddagger}, \theta\right)$ can be easily obtained using perturbation theory as will be described in Sec. 3.2.3. 
Angular momentum is included in the above formulae simply by noting that the classical Hamiltonian will, in general, also be a function of the magnitude of the angular momentum $J$ and its projection onto a body-fixed axis $K$. (If the molecule is not a symmetric top, $K$ is not a good quantum number but may still be used to label the rotational energy levels. In either case, there will be $F=3 N-6$ vibrational quantum numbers, for an $N$ atom non-linear system.) Eq. (2.16) is thus changed to read

$$
\mathcal{H}\left(n_{1}+\frac{1}{2}, n_{2}+\frac{1}{2}, \ldots, n_{F-1}+\frac{1}{2},-\frac{i}{\pi} \theta, J, K\right)=E
$$

which determines $\theta(E, n, J, K)$ and thereby the transmission probability is

$$
P_{\underline{n}, J, K}(E)=\left(1+\mathrm{e}^{2 \theta(\underline{n}, J, K ; E)}\right)^{-1} .
$$

Moreover, the Hamiltonian may be obtained quantum mechanically and use of the correspondence,

$$
\theta(\underset{\sim}{n}, J, K ; E)=\frac{\pi}{i}\left(n_{F}(\stackrel{n}{\sim}, J, K ; E)+\frac{1}{2}\right),
$$

leads to a Hamiltonian of the form in Eq. (2.17) which can then be inverted to obtain $P_{\underline{n}, J, K}(E)$.

\subsection{Perturbation Theory for the Activated Com- plex}

\subsubsection{General Theory}

Determining the Hamiltonian as a function of the good action variables requires an analytic solution of the classical equations of motion and is thus not possible, in general. It can, however, be accomplished to a useful level of approximation by perturbatively including anharmonicity, coriolis coupling, etc., in essentially the same way they are handled in determining rovibrational energy levels of a stable molecule (i.e., about a minimum on the potential energy surface.) Therefore the Hamiltonian is expanded at the transition state and only those terms which contribute to the second order eigenvalues are retained, i.e., the vibrational terms up to quartic order with 
respect to the mass weighted normal coordinates $Q_{k}$ of the transition state, the rigid rotor terms, and the rovibrational coupling terms arising from the coriolis interaction:

$$
\begin{aligned}
\mathcal{H}= & V_{0}+\frac{1}{2} \sum_{k}^{F}\left(-\frac{\partial^{2}}{\partial Q^{2}}+\omega_{k}^{2} Q_{k}^{2}\right)+\frac{1}{6} \sum_{k l m}^{F} f_{k l m} Q_{k} Q_{l} Q_{m}+\frac{1}{24} \sum_{k l m n}^{F} f_{k l m n} Q_{k} Q_{l} Q_{m} Q_{n} \\
& +\sum_{\beta} B_{\beta} J_{\beta}^{2}-2 \sum_{k, l}^{F} Q_{k} P_{l} \sum_{\beta} B_{\beta} \zeta_{k, l}^{\beta} J_{\beta}
\end{aligned}
$$

where $V_{0}$ is the potential energy at the saddle point, the sums over lowercase letters are unrestricted sums over the $F$ vibrational modes, and the sums over Greek letters are sums over the rotational axes. The primary differences from the standard rovibrational expansion ${ }^{107}$ are that at zeroth order the potential along the reaction coordinate (mode $F$ ) is a harmonic barrier vis-a-vis the frequency $\omega_{F}$ is imaginary,

$$
\omega_{F}=i\left|\omega_{F}\right| \equiv i \bar{\omega}_{F},
$$

and the action of the reactive partition in Eq. (2.19) is complex. The complex nature of these values will propagate throughout the perturbative expansion and so in the following sections these terms will be written out explicitly in order to show those that are imaginary and to show that the resulting perturbative eigenvalues are indeed real.

Using second order Van Vleck perturbation theory, ${ }^{107}$ the Hamiltonian in Eq. (2.20), is given in terms of the local good vibrational quantum numbers (or action variables, $I_{k}=n_{k}+\frac{1}{2}$ ) by

$$
\begin{aligned}
E= & V_{0}+\bar{E}_{0}+\sum_{k}^{F} \omega_{k}\left(n_{k}+\frac{1}{2}\right)+\sum_{k \leq l}^{F} x_{k l}\left(n_{k}+\frac{1}{2}\right)\left(n_{l}+\frac{1}{2}\right) \\
& +\sum_{\beta}\left[B_{\beta}-\sum_{k} \alpha_{k}^{\beta}\left(n_{k}+\frac{1}{2}\right)\right] J_{\beta}^{2},
\end{aligned}
$$

which is correct only if there are no degenerate vibrations. (Note that the reactive mode $F$ is assumed to be nondegenerate.) The result for doubly degenerate perpendicular vibrations is presented in Sec. 2.3.3 and it is particularly relevant for symmetric tops, e.g., the $\mathrm{H}+\mathrm{H}_{2}$ transition state, in which there necessarily exists such a degenerate pair. ${ }^{104}$ For almost-symmetric tops, this degeneracy is not enforced 
by symmetry and the vibrations can be treated through Eq. (2.22) while including rotation with symmetric top rotational levels. ${ }^{113}$

The rotational Hamiltonian in Eq. (2.22) can be diagonalized numerically for asymmetric tops. For approximateiy symmetric tops, it reduces to:

$$
\begin{aligned}
E= & V_{0}+\bar{E}_{0}+B_{\perp} J(J+1)+\left(B_{\|}-B_{\perp}\right) K^{2} \\
& +\sum_{k}^{F}\left[\omega_{k}-\alpha_{k}^{\perp} J(J+1)-\left(\alpha_{k}^{\|}-\alpha_{k}^{\perp}\right) K^{2}\right]\left(n_{k}+\frac{1}{2}\right) \\
& +\sum_{k \leq k^{\prime}}^{F} x_{k k^{\prime}}\left(n_{k}+\frac{1}{2}\right)\left(n_{k^{\prime}}+\frac{1}{2}\right)
\end{aligned}
$$

where $B_{\|}$and $\alpha_{k}^{\|}$correspond to the values of the unique axis, and $B_{\perp}$ and $\alpha_{k} \frac{1}{k}$ correspond to the average of the values of the perpendicular axes. Through the use of Eq. (2.19), this can be rewritten as a quadratic equation in $\theta$ with real coefficients and inverted to obtain:

$$
\theta(\underset{\sim}{n}, J, K ; E)=\frac{2 \pi \delta E / \tilde{\omega}_{F}}{1+\sqrt{1+4 x_{F F} \delta E / \tilde{\omega}_{F}^{2}}}
$$

where

$$
\begin{aligned}
\delta E \equiv & V_{0}-E+\bar{E}_{0}+B_{\perp} J(J+1)+\left(B_{\|}-B_{\perp}\right) K^{2} \\
& +\sum_{k=1}^{F-1}\left[\omega_{k}-\alpha_{k}^{\perp} J(J+1)-\left(\alpha_{k}^{\|}-\alpha_{k}^{\perp}\right) K^{2}\right]\left(n_{k}+\frac{1}{2}\right) \\
& +\sum_{k \leq k^{\prime}}^{F-1} x_{k k^{\prime}}\left(n_{k}+\frac{1}{2}\right)\left(n_{k^{\prime}}+\frac{1}{2}\right) \\
\tilde{\omega}_{F} \equiv & \bar{\omega}_{F}+i \alpha_{F}^{\perp} J(J+1)+i\left(\alpha_{F}^{\|}-\alpha_{F}^{\perp}\right) K^{2}-i \sum_{k=1}^{F-1} x_{k F}\left(n_{k}+\frac{1}{2}\right)
\end{aligned}
$$

$i=\sqrt{-1} ;$ and $\alpha_{F}^{\perp}, \alpha_{F}^{\|}, \alpha_{F}^{\perp}$, and $x_{k F}$ for $k=1, \ldots, F-1$ will be shown to be purely imaginary in the following sections. (The root, Eq. (2.24a), of the quadratic equation in $\theta, \mathrm{Eq} .(2.23)$, is chosen so that in the $x_{F F} \rightarrow 0$ limit, $\theta$ reduces to the standard harmonic result.)

Before proceeding to present explicit expressions for the anharmonic constants in Eqs. (2.22) and (2.24), it is interesting to note some qualitative aspects of 
the present expressions. For small $\delta E$ the generalized barrier penetration integral of Eq. (2.24a) is given by

$$
\theta(\underset{\sim}{n}, E) \approx \delta E\left[\frac{\pi}{\hbar \Omega_{F}}\right]-\delta E^{2}\left[\frac{\pi x_{F F}}{\left(\hbar \Omega_{F}\right)^{3}}\right]+\ldots
$$

from which one can see several features. The first term in Eq. (2.25) is the purely harmonic (i.e. parabolic barrier) expression ${ }^{114}$ for $\theta$, with the exception that the frequency $\Omega_{F}$ has been renormalized from the one-dimensional barrier frequency $\bar{\omega}_{F}$ by coupling to the $F-1$ other modes ( $c f$. Eq. 2.25). Modes $k$ for which $\bar{x}_{k F}$ is positive will decrease $\Omega_{F}$, which decreases the tunneling probability, and the converse is true for modes with negative $\bar{x}_{k F}$. The effect of the second term in Eq. (2.25) depends on the sign of $x_{F F}$, the diagonal anharmonic constant for the reaction coordinate mode: $x_{F F}>0$ decreases the value of $\theta$ and thus increases the tunneling probability, and vice versa if $x_{F F}<0$. Anticipating the result in Eq. $(2.28 \mathrm{~b})$, one sees that the one-dimensional anharmonicity (i.e. $f_{F F F F}$, which will typically be positive, and $f_{F F F}^{2}$ ) tends to make $x_{F F}$ negative, and thus to decrease the tunneling, while the last term in Eq. (2.28b), which results from coupling of the reaction coordinate to the other modes, tends to make $x_{F F}$ positive and thus increase tunneling. This last effect is that of reaction path curvature which is known in many different contexts ${ }^{115-119}$ to increase tunneling probabilities.

It should also be noted that this theory will break down when

$$
\frac{4 x_{F F} \delta E}{\tilde{\omega}_{F}^{2}}<-1
$$

which can result either from a resonance between the rovibrational modes or from too low an order in the perturbation treatment. In the former case, the offending resonant term can be treated at lower order and explicitly diagonalized. ${ }^{104}$ In the latter case, a higher order perturbative treatment is required and it can formally be carried out easily using symbolic manipulation with a computer. ${ }^{120}$ However, the higher order $a b$ initio rovibrational derivatives of the activated complex are not available and thus the higher order perturbative calculation is not within reach. 


\subsubsection{Nondegenerate Vibrational Modes}

If the Hamiltonian has no degenerate vibrational modes, the zeroth order states are simply direct products of $F-1$ one-dimensional harmonic oscillator states and a one-dimensional harmonic oscillator scattering state with complex argument. ${ }^{103,112}$ The degeneracy of the scattering state is lifted by imposing outgoing wave boundary conditions. Moreover, it satisfies the algebra of the raising and lowering operators, and hence one can readily use the results ${ }^{107}$ obtained for a regular harmonic oscillator direct product basis, modifying only the signs of those terms according to the number of imaginary terms which are now present.

The zeroth order constant energy correction ${ }^{121}$ accounting for the reaction coordinate is,

$$
\begin{aligned}
\bar{E}_{0}= & \frac{1}{64} \sum_{k}^{F-1} \frac{f_{k k k k}}{\omega_{k}^{2}}-\frac{7}{576} \sum_{k}^{F-1} \frac{f_{k k k}^{2}}{\omega_{k}^{4}}+\frac{3}{64} \sum_{k \neq l}^{F-1} \frac{f_{k l l}^{2}}{\left(4 \omega_{l}^{2}-\omega_{k}^{2}\right) \omega_{l}^{2}} \\
& -\frac{1}{4} \sum_{k<l<m}^{F-1} \frac{f_{k l m}^{2}}{\left[\left(\omega_{k}+\omega_{l}\right)^{2}-\omega_{m}^{2}\right]\left[\left(\omega_{k}-\omega_{l}\right)^{2}-\omega_{m}^{2}\right]} \\
& -\frac{1}{64} \frac{f_{F F F F}}{\bar{\omega}_{F}^{2}}-\frac{7}{576} \frac{f_{F F F}^{2}}{\bar{\omega}_{F}^{4}}+\frac{3}{64} \sum_{k}^{F-1} \frac{f_{k F F}^{2}}{\left(4 \bar{\omega}_{F}^{2}+\omega_{k}^{2}\right) \bar{\omega}_{F}^{2}}+\frac{3}{64} \sum_{k}^{F-1} \frac{f_{k k F}^{2}}{\left(4 \omega_{k}^{2}+\bar{\omega}_{F}^{2}\right) \omega_{k}^{2}} \\
& -\frac{1}{4} \sum_{k<l}^{F-1} \frac{f_{k l F}^{2}}{\left[\left(\omega_{k}+\omega_{l}\right)^{2}+\bar{\omega}_{F}^{2}\right]\left[\left(\omega_{k}-\omega_{l}\right)^{2}+\bar{\omega}_{F}^{2}\right]} \\
& -\frac{\hbar}{16 \pi c}\left(\frac{1}{I_{x x}}+\frac{1}{I_{y y}}+\frac{3}{I_{z z}}\right) .
\end{aligned}
$$

where $\underset{\sim}{I}$ is the moment of inertia tensor.

Similarly, the anharmonic terms with $k, l$ and $m$ running over all modes except for the reactive mode $F$, can be written explicitly as: ${ }^{103}$

$$
\begin{aligned}
x_{k k}= & \frac{\hbar^{2}}{16 \omega_{k}^{2}}\left\{f_{k k k k}+\frac{f_{k k F}^{2}\left(8 \omega_{k}^{2}+3 \bar{\omega}_{F}^{2}\right)}{\bar{\omega}_{F}^{2}\left(4 \omega_{k}^{2}+\bar{\omega}_{F}^{2}\right)}-\sum_{l}^{F-1} \frac{f_{k k l}^{2}\left(8 \omega_{k}^{2}-3 \omega_{l}^{2}\right)}{\omega_{l}^{2}\left(4 \omega_{k}^{F}-\omega_{l}^{2}\right)}\right\}, \\
x_{F F}= & -\frac{\hbar^{2}}{16 \bar{\omega}_{F}^{2}}\left\{f_{F F F F}+\frac{5 f_{F F F}^{2}}{3 \bar{\omega}_{F}^{2}}-\sum_{l}^{F-1} \frac{f_{F F l}^{2}\left(8 \bar{\omega}_{F}^{2}+3 \omega_{l}^{2}\right)}{\omega_{l}^{2}\left(4 \bar{\omega}_{F}^{2}+\omega_{l}^{2}\right)}\right\}, \\
x_{k l}= & \frac{\hbar^{2}}{4 \omega_{k} \omega_{l}}\left\{f_{k k l l}+\frac{f_{k k F} f_{l l F}}{\bar{\omega}_{F}^{2}}+\frac{2 f_{k l F}^{2}\left(\omega_{k}^{2}+\omega_{l}^{2}+\bar{\omega}_{F}^{2}\right)}{\left[\left(\omega_{k}+\omega_{l}\right)^{2}+\bar{\omega}_{F}^{2}\right]\left[\left(\omega_{k}-\omega_{l}\right)^{2}+\bar{\omega}_{F}^{2}\right]}\right. \\
& \left.-\sum_{m}^{F-1} \frac{f_{k k m} f_{l l m}}{\omega_{m}^{2}}+\sum_{m}^{F-1} \frac{2 f_{k l m}^{2}\left(\omega_{k}^{2}+\omega_{l}^{2}-\omega_{m}^{2}\right)}{\left[\left(\omega_{k}+\omega_{l}\right)^{2}-\omega_{m}^{2}\right]\left[\left(\omega_{k}-\omega_{l}\right)^{2}-\omega_{m}^{2}\right]}\right\}
\end{aligned}
$$




$$
\begin{aligned}
& +\left(\frac{\omega_{k}}{\omega_{l}}+\frac{\omega_{l}}{\omega_{k}}\right) \sum_{\alpha} B_{\alpha}\left(\zeta_{k l}^{\alpha}\right)^{2} \\
x_{k F}= & -\frac{i \hbar^{2}}{4 \omega_{k} \bar{\omega}_{F}}\left\{f_{k k F F}+\frac{f_{k k F} f_{F F F}}{\bar{\omega}_{F}^{2}}+\frac{2 f_{k F F}^{2}}{\omega_{k}^{2}+4 \bar{\omega}_{F}^{2}}-\sum_{m}^{F-1} \frac{f_{k k m} f_{F F m}}{\omega_{m}^{2}}\right. \\
& \left.+\sum_{m}^{F-1} \frac{2 f_{k F m}^{2}\left(\omega_{k}^{2}-\bar{\omega}_{F}^{2}-\omega_{m}^{2}\right)}{\left[\left(\omega_{k}+\omega_{m}\right)^{2}+\bar{\omega}_{F}^{2}\right]\left[\left(\omega_{k}-\omega_{m}\right)^{2}+\bar{\omega}_{F}^{2}\right]}\right\} \\
& +\left(\frac{\omega_{k}}{\bar{\omega}_{F}}-\frac{\bar{\omega}_{F}}{\omega_{k}}\right) \sum_{\alpha} B_{\alpha}\left(\zeta_{k F}^{\alpha}\right)^{2} .
\end{aligned}
$$

Since, $x_{k r}$ is purely imaginary, it is convenient to define the purely real coupling between the perpendicular mode $k$ and the generalize barrier penetration integral as:

$$
x_{k r} \equiv-i \bar{x}_{k F} .
$$

Substitution of these expressions into Eq. (2.24c) demonstrates explicitly that the vibrational anharmonic terms are indeed real.

\subsubsection{Degenerate Vibrational Modes}

Because symmetric tops are ubiquitous, and because they neccessarily have at least one degenerate pair of vibrational modes, one must also consider transition states with degenerate vibrational modes. For simplicity, we discuss only the case with doubly degenerate modes as it illustrates all the new physics and it is also the most general case which will be encountered in the applications of this and subsequent chapters. In this case, the harmonic oscillator direct product zeroth-order basis used in the previous section to describe the modes perpendicular to the reactive modes is not applicable; instead the degenerate vibrations must be treated as a 2dimensional harmonic oscillator, and the perturbation theory done anew. (Note that since the transition state, by assumption, contains only one direction with an imaginary frequency, degeneracy can only occur in the perpendicular vibrational modes.) However, the perturbative treatment of a bound system with doubly degenerate vibrational modes is also a standard procedure, ${ }^{107}$ and as before one need only take care in substituting the imaginary quantities associated with the activated complex into the standard formulae. 
The perturbative eigenenergies in Eq. (2.22) are now replaced by

$$
\begin{aligned}
E= & V_{0}+\bar{E}_{0}^{\prime}+\sum_{k} \omega_{k}\left(n_{k}+\frac{1}{2}\right)+\sum_{s} \omega_{s}\left(n_{s}+1\right)+\sum_{k \leq l}^{F} x_{k l}\left(n_{k}+\frac{1}{2}\right)\left(n_{l}+\frac{1}{2}\right) \\
& +\sum_{s \leq t}^{F} x_{s t}\left(n_{s}+1\right)\left(n_{t}+1\right)+\sum_{k, s}^{F} x_{k s}\left(n_{k}+\frac{1}{2}\right)\left(n_{s}+1\right)+\sum_{s \leq t} g_{s t} \mathbf{l}_{s} \mathbf{l}_{t} \\
& +\sum_{\beta}\left[B_{\beta}-\sum_{k} \alpha_{k}^{\beta}\left(n_{k}+\frac{1}{2}\right)-\sum_{s} \alpha_{s}^{\beta}\left(n_{s}+1\right)\right] J_{\beta}^{2} .
\end{aligned}
$$

where the terms have been divided according to the following classes of modes: $\{k, l, m\}$ label nondegenerate perpendicular vibrational modes, $\{s, t, u\}$ label doubly degenerate perpendicular vibrational modes, and $F$ is the reactive mode. Also recall that doubly degenerate vibrational modes have an additional quantum number 1 , here labelled by $\{a, b\}$, which corresponds to the vibrational angular momentum of the two-dimensional harmonic oscillator.

The zeroth order energy correction ${ }^{122}$ accounting for the reaction coordinate and for doubly degenerate vibrational modes is

$$
\begin{aligned}
\bar{E}_{0}^{\prime}= & \bar{E}_{0}+\frac{1}{48} \sum_{s} \frac{f_{s s s s}}{\omega_{s}^{2}}+\frac{11}{144} \sum_{s} \frac{f_{s s s}^{2}}{\omega_{s}^{4}}+\frac{1}{16} \sum_{k, s} \frac{f_{k s s}^{2}}{\left(4 \omega_{s}^{2}-\omega_{k}^{2}\right) \omega_{s}^{2}} \\
& -\frac{1}{2} \sum_{s<t} \sum_{k} \frac{f_{k s t}^{2}}{\left[\left(\omega_{s}+\omega_{t}\right)^{2}-\omega_{k}^{2}\right]\left[\left(\omega_{s}-\omega_{t}\right)^{2}-\omega_{k}^{2}\right]}+\frac{1}{16} \sum_{s \neq t} \frac{f_{s s t}^{2}\left(8 \omega_{s}^{2}+\omega_{t}^{2}\right)}{\omega_{s}^{2} \omega_{t}^{2}\left(4 \omega_{s}^{2}-\omega_{t}^{2}\right)} \\
& -\sum_{s<t, u} \frac{2 f_{s t u}^{2}}{\left[\left(\omega_{s}+\omega_{t}\right)^{2}-\omega_{u}^{2}\right]\left[\left(\omega_{s}-\omega_{t}\right)^{2}-\omega_{u}^{2}\right]}+\frac{1}{16} \sum_{s} \frac{f_{s s F}^{2}}{\left(4 \omega_{s}^{2}+\bar{\omega}_{F}^{2}\right) \omega_{s}^{2}} \\
& -\frac{1}{2} \sum_{s<t} \frac{f_{s t F}^{2}}{\left[\left(\omega_{s}+\omega_{t}\right)^{2}+\bar{\omega}_{F}^{2}\right]\left[\left(\omega_{s}-\omega_{t}\right)^{2}+\bar{\omega}_{F}^{2}\right]}
\end{aligned}
$$

where $\bar{E}_{0}$ is the non-degenerate result in Eq. (2.27).

The non-degenerate anharmonic terms presented in Eq. (2.28) are also correct for the doubly degenerate case; the remaining terms can be readily obtained:

$$
\begin{aligned}
x_{s t}= & \frac{\hbar^{2}}{8 \omega_{s} \omega_{t}}\left\{f_{s a s a t a t a}+f_{s a s a t b t b}+2 \frac{f_{s s F} f_{t t F}}{\bar{\omega}_{F}^{2}}+\frac{2 f_{s t F}^{2}\left(\omega_{s}^{2}+\omega_{t}^{2}+\bar{\omega}_{F}^{2}\right)}{\left[\left(\omega_{s}+\omega_{t}\right)^{2}+\bar{\omega}_{F}^{2}\right]\left[\left(\omega_{s}-\omega_{t}\right)^{2}+\bar{\omega}_{F}^{2}\right]}\right. \\
& -2 \sum_{m} \frac{f_{s s m} f_{t t m}}{\omega_{m}^{2}}+2 \sum_{m} \frac{f_{s t m}^{2}\left(\omega_{s}^{2}+\omega_{t}^{2}-\omega_{m}^{2}\right)}{\left[\left(\omega_{s}+\omega_{t}\right)^{2}-\omega_{m}^{2}\right]\left[\left(\omega_{s}-\omega_{t}\right)^{2}-\omega_{m}^{2}\right]} \\
& \left.+4 \sum_{u} \frac{f_{s t u}^{2}\left(\omega_{s}^{2}+\omega_{t}^{2}-\omega_{u}^{2}\right)}{\left[\left(\omega_{s}+\omega_{t}\right)^{2}-\omega_{u}^{2}\right]\left[\left(\omega_{s}-\omega_{t}\right)^{2}-\omega_{u}^{2}\right]}\right\}
\end{aligned}
$$




$$
\begin{aligned}
& +\left(\frac{\omega_{0}}{\omega_{t}}+\frac{\omega_{t}}{\omega_{0}}\right)\left[\frac{1}{2} B_{z}\left(\zeta_{s a t b}^{z}\right)^{2}+B_{x}\left(\zeta_{s a t a}^{y}\right)^{2}\right], \\
& x_{s s}=\frac{\hbar^{2}}{16 \omega_{s}^{2}}\left\{f_{s s s s}+\frac{f_{s s F}^{2}\left(8 \omega_{s}^{2}+3 \bar{\omega}_{F}^{2}\right)}{\bar{\omega}_{F}^{2}\left(4 \omega_{s}^{2}+\bar{\omega}_{F}^{2}\right)}\right. \\
& \left.-\sum_{m} \frac{f_{s s m}^{2}\left(8 \omega_{s}^{2}-3 \omega_{m}^{2}\right)}{\omega_{m}^{2}\left(4 \omega_{s}^{2}-\omega_{m}^{2}\right)}-\sum_{t} \frac{f_{s s t}^{2}\left(8 \omega_{s}^{2}-3 \omega_{t}^{2}\right)}{\omega_{t}^{2}\left(4 \omega_{s}^{2}-\omega_{t}^{2}\right)}\right\} \\
& x_{k s}=\frac{\hbar^{2}}{4 \omega_{k} \omega_{s}}\left\{f_{k k s s}+\sum_{u} \frac{f_{k s u}^{2}\left(\omega_{k}^{2}+\omega_{s}^{2}-\omega_{u}^{2}\right)}{\left[\left(\omega_{k}+\omega_{s}\right)^{2}-\omega_{u}^{2}\right]\left[\left(\omega_{k}-\omega_{s}\right)^{2}-\omega_{u}^{2}\right]}\right. \\
& \left.+\frac{f_{k k F} f_{s s F}}{\bar{\omega}_{F}^{2}}-\sum_{m} \frac{f_{k k m} f_{s s m}}{\omega_{m}^{2}}\right\}+\left(\frac{\omega_{s}}{\omega_{t}}+\frac{\omega_{s}}{\omega_{s}}\right) B_{x}\left[\left(\zeta_{k s}^{x}\right)^{2}+\left(\zeta_{k s}^{y}\right)^{2}\right] \text {, } \\
& x_{\text {SFF }}=-\frac{i \hbar^{2}}{4 \omega_{s} \bar{\omega}_{F}}\left\{f_{\text {saFF }}+\sum_{u} \frac{2 f_{s F u}^{2}\left(\omega_{s}^{2}-\bar{\omega}_{F}^{2}-\omega_{u}^{2}\right)}{\left[\left(\omega_{s}+\omega_{u}\right)^{2}+\bar{\omega}_{F}^{2}\right]\left[\left(\omega_{s}-\omega_{u}\right)^{2}+\bar{\omega}_{F}^{2}\right]}\right. \\
& \left.+\frac{f_{s s F} f_{F F F}}{\bar{\omega}_{F}^{2}}-\sum_{m} \frac{f_{s s m} f_{F F m}}{\omega_{m}^{2}}\right\}+\left(\frac{\omega_{g}}{\bar{\omega}_{F}}-\frac{\bar{\omega}_{F}}{\omega_{s}}\right) B_{x}\left[\left(\zeta_{F s}^{x}\right)^{2}+\left(\zeta_{F s}^{y}\right)^{2}\right] \text {, } \\
& g_{s s}=-\frac{\hbar^{2}}{48 \omega_{s}^{2}}\left\{f_{s s s s}-\frac{3 f_{s s F}^{2}}{\left(4 \omega_{s}^{2}+\bar{\omega}_{F}^{2}\right)}+3 \sum_{m} \frac{f_{s s m}^{2}}{\left(4 \omega_{s}^{F}-\omega_{m}^{2}\right)}\right. \\
& \left.-\sum_{u} \frac{f_{s s u}^{2}\left(8 \omega_{s}^{2}-\omega_{u}^{2}\right)}{\omega_{t}^{2}\left(4 \omega_{s}^{2}-\omega_{u}^{2}\right)}\right\}+B_{z}\left(\zeta_{x s}^{z}\right)^{2} \\
& g_{s t}=\frac{1}{2} \frac{f_{s t F}^{2}}{\left[\left(\omega_{s}+\omega_{t}\right)^{2}+\bar{\omega}_{F}^{2}\right]\left[\left(\omega_{s}-\omega_{t}\right)^{2}+\bar{\omega}_{F}^{2}\right]} \\
& +\frac{1}{2} \sum_{m} \frac{f_{s t m}^{2}}{\left[\left(\omega_{s}+\omega_{t}\right)^{2}-\omega_{m}^{2}\right]\left[\left(\omega_{s}-\omega_{t}\right)^{2}-\omega_{m}^{2}\right]} \\
& -\sum_{u} \frac{f_{s t u}^{2}}{\left[\left(\omega_{s}+\omega_{t}\right)^{2}-\omega_{u}^{2}\right]\left[\left(\omega_{s}-\omega_{t}\right)^{2}-\omega_{u}^{2}\right]} \\
& -2 B_{x}\left(\zeta_{s t}^{y}\right)^{2}+B_{z}\left(\zeta_{s t}^{z}\right)^{2}+2 B_{z} \zeta_{s s}^{z} \zeta_{t t}^{z} \text {. }
\end{aligned}
$$

As is the case for the $x_{k F}$ term of the previous section, here the $x_{s F}$ term is purely imaginary, and suggests the definition,

$$
x_{k F} \equiv-i \bar{x}_{k F}
$$

Substitution of the anharmonicities here obtained into the degenerate vibrational pertubartive Hamiltonian, Eq. (2.30), explicitly demonstrates that the eigenenergies are indeed real. 


\subsubsection{Rotations}

The rotational constant for mode $F$ is also imaginary,

$$
\alpha_{F}^{\beta}=i \bar{\alpha}_{F}^{\beta},
$$

and modification of the standard rovibrational expression ${ }^{107}$ yields the real quantity,

$$
\begin{aligned}
\bar{\alpha}_{F}^{\beta}= & \left(\frac{2 B_{\beta}^{2}}{\bar{\omega}_{F}}\right)\left[\sum_{\gamma}\left(\frac{3\left(a_{F}^{\beta \gamma}\right)^{2}}{4 I_{\gamma \gamma}}\right)-\sum_{l}^{F-1}\left(\zeta_{F l}^{\beta}\right)^{2}\left(\frac{\omega_{l}^{2}-3 \bar{\omega}_{F}^{2}}{\omega_{l}^{2}+\bar{\omega}_{F}^{2}}\right)\right. \\
& \left.-\pi\left(\frac{c}{\hbar}\right)^{1 / 2}\left(\frac{f_{F F F} a_{F}^{\beta \beta}}{\bar{\omega}_{F}^{2}}-\sum_{l}^{F-1} \frac{f_{F F l} a_{l}^{\beta \beta}}{\omega_{l}^{2}}\right)\right],
\end{aligned}
$$

where $a^{\beta \gamma}$ is the first derivative of the moment of inertia tensor, $\underset{\tilde{r}}{I}$, with respect to the normal mode coordinates. The remaining rotational constants are slight modifications of the standard result, ${ }^{107}$

$$
\begin{aligned}
\alpha_{k}^{\beta}= & -\left(\frac{2 B_{\beta}^{2}}{\omega_{k}}\right)\left[\sum_{\gamma}\left(\frac{3\left(a_{k}^{\beta \gamma}\right)^{2}}{4 I_{\gamma \gamma}}\right)+\sum_{l \neq k}^{F-1}\left(\zeta_{k l}^{\beta}\right)^{2}\left(\frac{3 \omega_{k}^{2}+\omega_{l}^{2}}{\omega_{k}^{2}-\omega_{l}^{2}}\right)\right. \\
& \left.+\left(\zeta_{k F}^{\beta}\right)^{2}\left(\frac{3 \omega_{k}^{2}-\bar{\omega}_{F}^{2}}{\omega_{k}^{2}+\bar{\omega}_{F}^{2}}\right)-\pi\left(\frac{c}{\hbar}\right)^{1 / 2}\left(\frac{f_{k k F} a_{F}^{\beta \beta}}{\bar{\omega}_{F}^{2}}-\sum_{l}^{F-1} \frac{f_{k k l} a_{l}^{\beta \beta}}{\omega_{l}^{2}}\right)\right] .
\end{aligned}
$$

If the molecule has a degenerate vibration then the $\alpha_{k}^{\beta}$ and $\alpha_{s}^{\beta}$ may be readily obtained from the standard expressions ${ }^{107}$ in a similar fashion. The conclusion to be drawn from Eqs. (2.35) and (2.36) is that coriolis coupling can be readily included into the formalism, and that the resulting perturbative eigenvalues of the Hamiltonian, Eq. (2.17), remain real.

\subsubsection{Summary}

In this section, we have presented the details of the construction of the perturbative Hamiltonian necessary for application to a rovibrational reactive system. Because of the analogy between the perturbative Hamiltonian of the activated complex and that of a bound molecule, the constants appearing in the former will be referred to as the "spectroscopic constants" of the activated complex. The standard rovibrational expressions have been written explicitly in order to account for the 
imaginary frequency of the reactive degree of freedom and to prove that the resulting perturbative eigenenergies contain only real terms. In actual applications, it is simpler to take advantage of the complex arithmetic in ANSI FORTRAN, and program the expressions in general form. This suggests that existing higher order perturbation theory programs ${ }^{123,124}$ could be readily adapted for use with the SCTST, if higher order derivatives of the transition state are known.

Fermi resonances among the vibrational modes may and will arise, e.g., the classic $2 \omega_{s} \approx \omega_{k}$ resonance ${ }^{125}$ in symmetric tops affects $x_{s s}, x_{k s}$ and $g_{s s}$. The diagonal terms are obtained using the expressions in the preceding sections with the $x$ 's modified to exclude the resonant partial fraction. While there are now off-diagonal terms, only the matrix elements of the resonance operator coupling only the states of a given polyad are non-trivial. The energies of these states are obtained by diagonalizing the corresponding block of the Hamiltonian. ${ }^{107}$ In the same way, amended vibrational energy levels at the transition state can be obtained and used to calculate transmission probabilities. Note that there is no danger of a resonance developing between the reactive mode and any of the perpendicular modes as the reaction frequency is imaginary.

The only new difficulty presented by the presence of Fermi resonances in the application to the activated complex is that each diagonal entry of a given polyad is non-linearly coupled to $\theta$. Thus the simple quadratic formula in Eq. (2.24) is no longer correct, and the transmission probabilities for the polyad states need be obtained by numerical diagonalization and subsequent numerical inversion. However, in previous work $^{104}$ and in the work of Cohen et al., ${ }^{126}$ this difficulty has been approximately circumvented by diagonalizing the polyads at $\theta=0$ and using an effective linear coupling between the polyad states and the reactive mode $\theta$. This approximation readily allows the use of the quadratic expression for the transmission probability, and is therefore much easier to implement. The results under this approximation for the $\mathrm{H}+\mathrm{H}_{2}$ reaction are in reasonable agreement with those which will be shown in the subsequent section for the case in which the transmission probabilities are obtained without recourse to the approximation. 


\begin{tabular}{ccc}
\hline & $(i)$ & $(i i)$ \\
\hline$\omega_{1}$ & 2066.9 & 2066.9 \\
$\omega_{2}$ & 899.5 & 2066.5 \\
$\omega_{4}$ & $1493.1 i$ & $2066.1 i$ \\
$x_{11}$ & -25.4 & -25.4 \\
$x_{12}$ & 455.3 & -120.7 \\
$x_{14}$ & $-699.1 i$ & $-699.1 i$ \\
$x_{22}$ & -80.8 & 60.7 \\
$x_{24}$ & $107.7 i$ & $107.6 i$ \\
$x_{44}$ & -246.2 & -246.2 \\
$g_{22}$ & 74.7 & -38.2 \\
$\bar{E}_{0}$ & -279.84 & -138.32 \\
\hline \hline
\end{tabular}

Table 2.1: The table contains the "spectroscopic constants" of the $\mathrm{H}+\mathrm{H}_{2}$ activated complex either (i) including the near resonance, $\omega_{1} \approx 2 \omega_{2}$ or (ii) removing the resonance. ${ }^{104}$ In case (ii) the value of the force constant coupling the polyad states is also needed and it is: $f_{144}=-2.2839 \times 10^{8}$. Note that $\omega_{2}=\omega_{3}$ and $x_{2 k}=x_{3 k}$ for all $k$, and all values are in units of $\mathrm{cm}^{-1}$.

\subsection{Illustrative Examples}

In this section, SCTST transmission probabilities and CRPs are obtained for several reactive systems in order to explore the effect of including the nonseparability of the Hamiltonian at the transition state and to demonstrate the relative accuracy of the method as compared to exact results, where possible.

In Secs. 2.4.1 through 2.4.3, the $(J=0) \mathrm{H}+\mathrm{H}_{2}$ reaction is studied by treating the reaction either as occurring on a one-dimensional Eckart barrier, the collinear arrangement on the DMBE surface or on the full-dimensional DMBE surface. (Note that the DMBE surface is the double many-body expansion fit to $a b$ initio points by Varandas et $a .^{127}$ ) The force constants were obtained by finite differences, and use of the symmetric top equations in Sec. 2.3.3 readily provide the "spectroscopic constants" listed in Table 2.1 (Ref. 104). Because of the Fermi resonance between the degenerate bend (modes 2 and 3 ) and the symmetric stretch (mode 1), the "spectroscopic" constants obtained either by including or removing the resonance term are 
presented. The treatment of the Fermi resonance affects only the constants involved in the full-dimensional calculation and will consequently play a role only in Sec. 2.4.3. Some representative transmission probabilities for the $\mathrm{D}_{2} \mathrm{CO}$ dissociation are presented in Sec. 2.4.4 for the $J=0$ states of the activated complex. The nonzero angular momentum states are necessary for the application to the probability decay rate distributions in Chapter 6, and will be discussed in that context. For further applications including nonzero angular momentum, the reader is referred to Ref. 126.

\subsubsection{Eckart Barrier}

As an example of the results that can be obtained using the SCTST presented in the previous section, we first treat the dynamics of a one-dimensional Eckart barrier. The symmetric Eckart barrier ${ }^{128}$ can be written as

$$
V(x)=V_{0} \operatorname{sech}^{2}(\pi x / a),
$$

where the parameters $V_{0}$ and $a$ characterize the barrier height and length scale, respectively. In order to make connection with the $\mathrm{H}+\mathrm{H}_{2}$ reaction, these parameters $\left(V_{0}=.425 \mathrm{eV}, a=4.22 \times 10^{-9} \mathrm{~m}\right)$ are chosen so as to match the quadratic and quartic derivatives at the transition state of the DMBE surface with respect to the asymmetric stretch (mode 4) and the mass of the effective particle is also taken to match that mode 4 ( $\mu=1060$ a.u.) as was done by Seideman and Miller. ${ }^{129}$ (Note that a more general set of parameters for the Eckart barrier will be considered in Sec. 3.3.1 in the context of evaluating SCTST thermal rate constants.) In Fig. 2.1 the Eckart potential corresponding to the one-dimensional $\mathrm{H}+\mathrm{H}_{2}$ reaction path is compared to its quadratic (harmonic) and quartic (anharmonic) expansions. In the remaining panels of the figure, the transmission probability obtained by the exact quantum mechanical result ${ }^{128}$ for this Eckart potential is compared to the harmonic and anharmonic SCTST results.

Several important points about the effect of one-dimensional anharmonicity are illustrated in Fig. 2.1. Note, for example, that the transmission probabilities decrease upon inclusion of anharmonicity; this results from the thickening of the barrier 


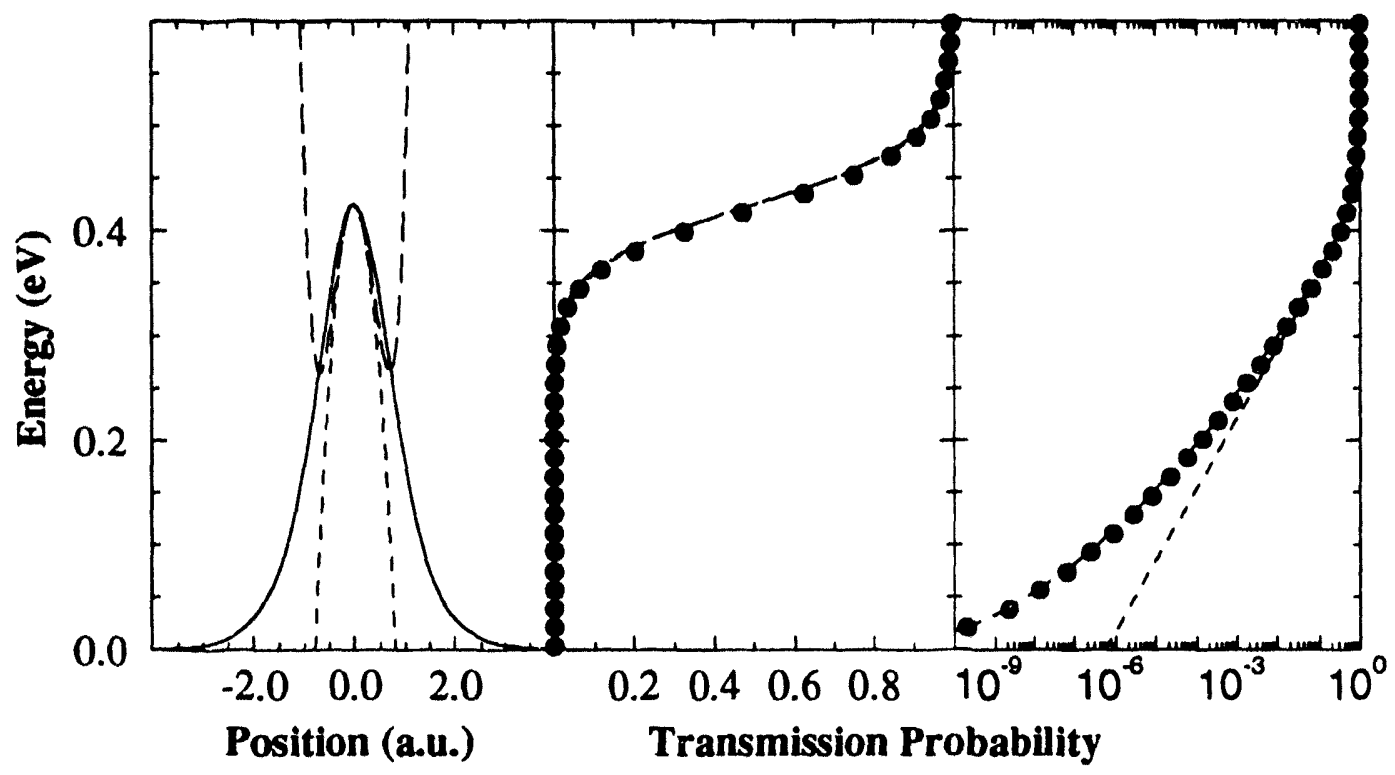

Figure 2.1: In the panel on the left, the Eckart potential with the parameters set to match the $\mathrm{H}+\mathrm{H}_{2}$ reaction is compared to its harmonic (short-dashed) and quartic (long-dashed) expansion. In the remaining panels, the transmission probability through the barrier is presented for the exact calculation ${ }^{128}$ (solid dots), the harmonic SCTST (short-dashed) and the anharmonic SCTST (long-dashed). The semi--log plot in the panel on the right emphasizes the agreement between the exact and anharmonic calculations in the low energy regime. 
due to the positive sign of $x_{F F}$. It should be clear that the quartic barrier potential corresponding to the expansion of the exact potential would produce, if solved exactly, incorrect results. (This can be seen either semiclassically, since the barrier penetration integral would have no turning points below the minima of the quartic potential, or quantum mechanically, since the quartic potential does not contain a continuous set of eigenstates.) Here the anharmonic SCTST is in remarkably good agreement with the exact results obtained for the exact potential. This is a consequence of the fact that the perturbative treatment in the SCTST is at a concomitant order to that of the potential expansion - that is, with respect to the perturbation theory, the Eckart barrier and it's quartic expansion are the same. A higher order perturbative treatment would produce worse results if no further information - higher-order potential derivatives - about the barrier were obtained.

\subsubsection{Collinear $\mathrm{H}+\mathrm{H}_{2} \rightarrow \mathrm{H}_{2}+\mathrm{H}$}

The collinear version of the $\mathrm{H}+\mathrm{H}_{2}$ reaction involves only modes 1 and 4, i.e., the symmetric and asymmetric stretches of the $\mathrm{H}_{3}$ collinear transition state. In this case there is no question of Fermi resonance, and the only nonseparable effects arise because of the anharmonicity in the barrier and the coupling between the two modes (reaction path curvature). Figure 2.2 shows the CRP in the low energy tunneling regime, as given by our full semiclassical theory, compared to the exact quantum results $^{2}$ and also to the harmonic limit of the theory. The "spectroscopic constants" used for these results may be found in Table 2.1, though with $\bar{E}_{0}$ set to zero. In Chapter 4, we will return to the issue of the proper evaluation of this value.

One sees that the present perturbative anharmonic theory does well, far into the deep tunneling regime. Although not shown in Fig. 2.2, at higher energies the contribution to the CRP arising from transition states with quanta in the perpendicular mode did not converge and this is because the large anharmonicity in the perpendicular mode causes the argument of the square root in equation (10) to be negative. However, in a system with more degrees of freedom the breakdown in the perturbation is mitigated by a cancellation of the anharmonicities in the various per- 


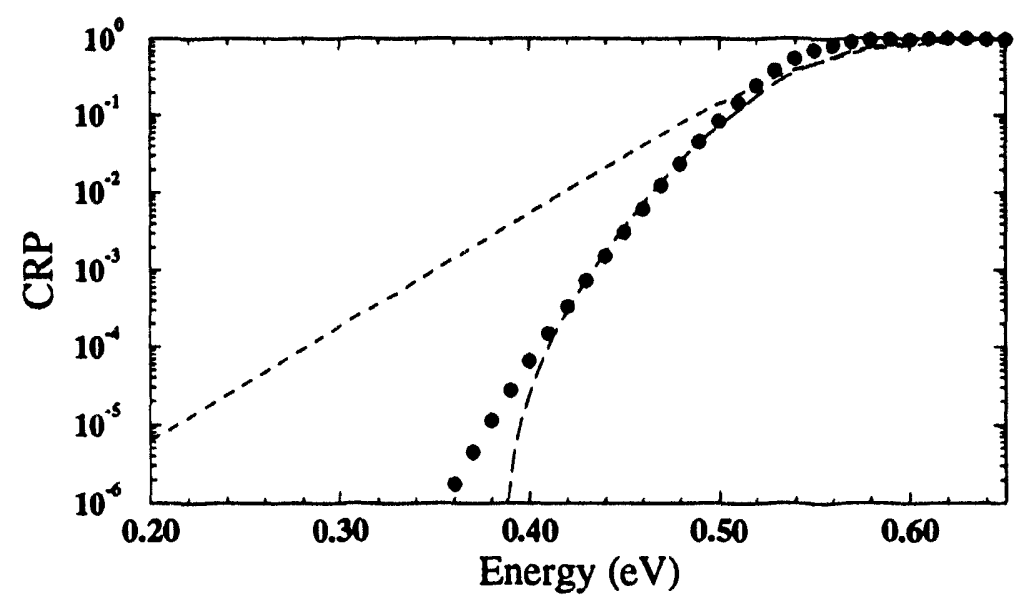

Figure 2.2: Cumulative reaction probability for collinear $\mathrm{H}+\mathrm{H}_{2}$. The points correspond to the exact quantum calculations of Bondi and Connor. ${ }^{2}$ The long-dashed curve is obtained by including the anharmonic terms in the semiclassical model. The short-dashed curve is obtained by including only the harmonic terms.

pendicular modes. Thus we expect that the present anharmonic theory will improve as the degrees of freedom increase.

\subsubsection{Full-dimensional $\mathrm{H}+\mathrm{H}_{2} \rightarrow \mathrm{H}_{2}+\mathrm{H}$}

The SCTST CRP for the $\mathrm{H}+\mathrm{H}_{2}$ reaction in full-dimensionality is compared to the exact CRP in Fig. 2.3. It is apparent that the anharmonic SCTST results obtained using the anharmonicities including the Fermi resonance term is actually in better agreement with the exact results than the anharmonic SCTST results for which the Fermi resonance terms are excluded and the polyads matrices are diagonalized. This result is surprising because the opposite conclusion was observed in earlier work $^{100,126}$ in which the polyad matrices were only approximately diagonalized. Inspection of the polyads suggests that this is a consequence of level repulsion between the diagonal energies which provides larger shifts than that which was obtained in the earlier studies.

In order to further explore the agreement between the SCTST and exact results, the Boltzmann weighted $\left.C R P, \exp (-\beta E) \times N_{(}^{\prime} E\right)$, for several temperatures 


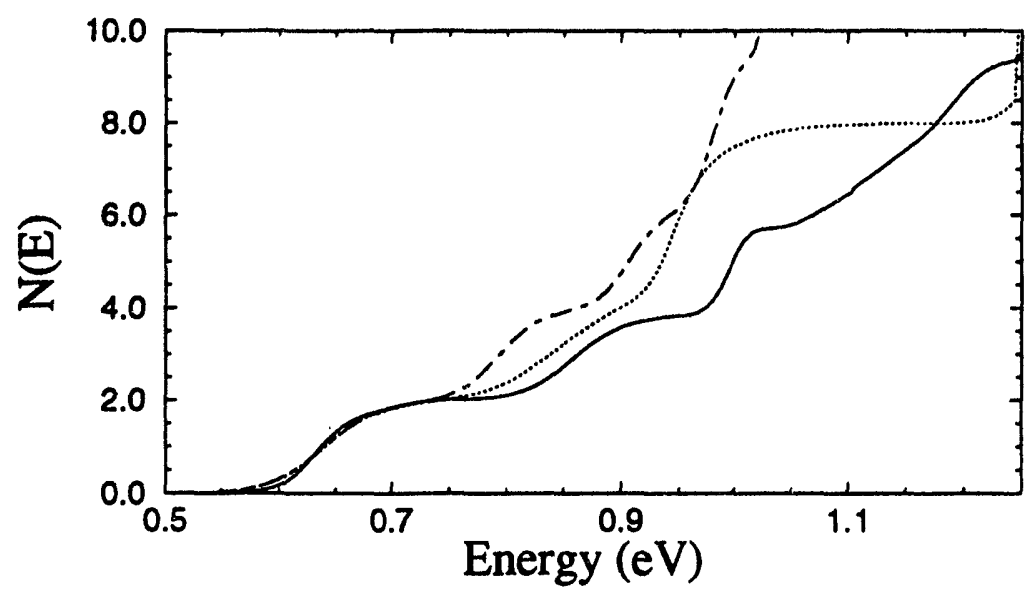

Figure 2.3: Cumulative reaction probability (CRP) for the full-dimensional $\mathrm{H}+\mathrm{H}_{2}$ reaction. The solid curve is the exact result of Seideman and Miller. ${ }^{130}$ The dotted curve is the anharmonic SCTST result using the "spectroscopic constants" in column (i) of Table 2.1 corresponding to the inclusion of the Fermi resonant terms. The dot-dash curve is obtained for column (ii) corresponding to the removal of the Fermi resonant terms. In both SCTST cases, the CRP is multiplied by the symmetry number $(\sigma=2)$ in order to account for the two possible exit channels (See, Sec. 3.3.2).

in the tunneling regime are illustrated in Fig. 2.4. The SCTST results are generally in good agreement with the exact results, displaying many of the same qualitative features and with values of the correct order of magnitude. The anharmonic SCTST results including the Fermi resonance in the "spectroscopic constants" is again in the best agreement with the exact results. Nonetheless, the remarkable agreement between the SCTST for this extremely anharmonic system is encouraging, as it suggests that for typical systems the anharmonic SCTST can certainly provide qualitative, if not quantitative, estimates of fairly detailed dynamical quantities such as the CRP.

\subsection{4 $\mathrm{D}_{2} \mathrm{CO} \rightarrow \mathrm{D}_{2}+\mathrm{CO}$}

The unimolecular dissociation of formaldehyde,

$$
\mathrm{D}_{2} \mathrm{CO} \rightarrow \mathrm{D}_{2}+\mathrm{CO},
$$

will be the focus of study in Chapter 6 in which RM/TST decay rate distributions are obtained and compared to the experimental results of Polik et al. ${ }^{21}$ Here we explore 

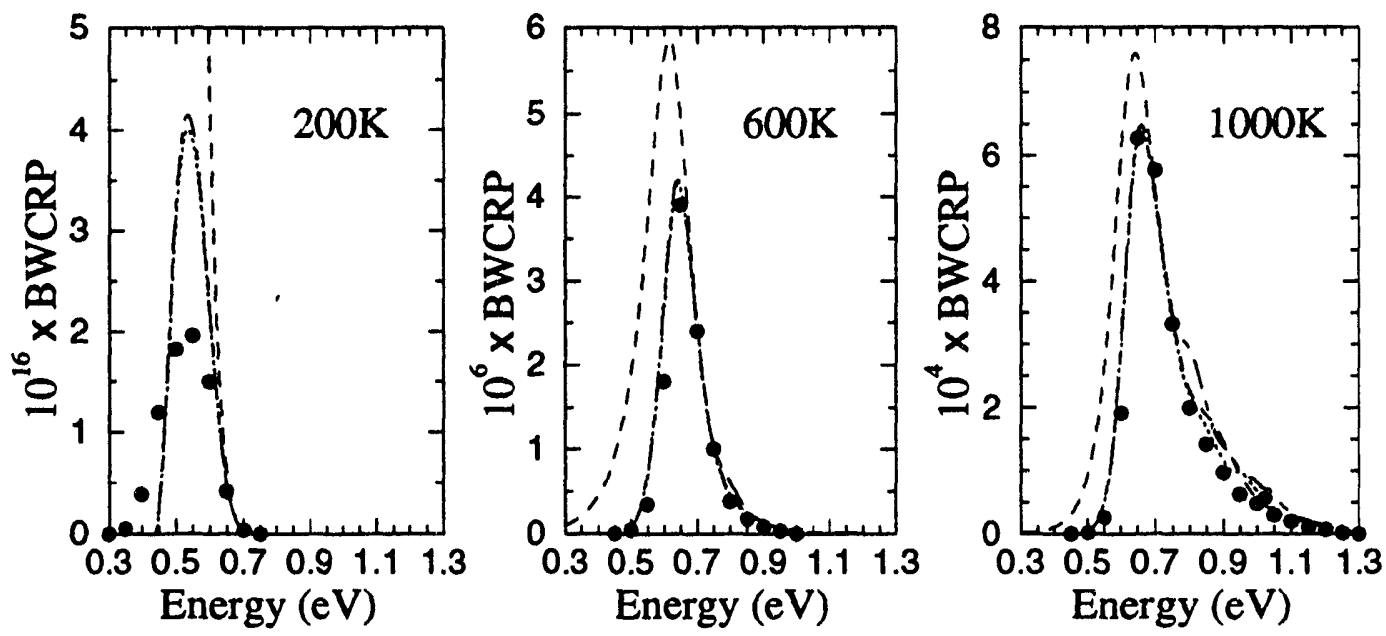

Figure 2.4: Boltzmann weighted cumulative reaction probabilities $\left(=\mathrm{e}^{-\beta E} N(E)\right)$ for the full-dimensional $\mathrm{H}+\mathrm{H}_{2}$ reaction are presented for various temperatures, $T=$ $200 \mathrm{~K}, 600 \mathrm{~K}$, and $1000 \mathrm{~K}$. In all cases, the solid points are the exact results of Chatfield et al., ${ }^{1}$ the short-dashed curve is the harmonic SCTST result, and the dotted (long-dashed) curves are the anharmonic SCTST results using the "spectroscopic terms" which include (remove) the Fermi resonance.

the transmission probabilities of the activated complex as an example of the use of SCTST to the unimolecular dissociation of a relatively large molecule. Attention is drawn to the paper by Gray et al., ${ }^{131}$ where a theoretical treatment of the tunneling dynamics is presented using a reaction path Hamiltonian model. ${ }^{132}$ In that treatment it was found that anharmonic corrections had a small effect on the unimolecular rate constants for the dissociation (2.38). The $a b$ initio calculation of the vibrational "spectroscopic constants" of the activated complex are described in Ref. 103, and the values are presented in Table 6.1 of Chapter 6.

Figures 2.5 and 2.6 present the SCTST transmission probabilities as a function of energy for several states $\underset{\sim}{n}$ of the activated complex. The two curves in each case correspond to the anharmonic model given by Eq. (2.24) (long-dashed curves) and the harmonic approximation to it, i.e. $x_{k l}=x_{k F}=x_{F F}=0$ (short-dashed curves). For the ground state of the activated complex one sees that the anharmonic corrections are very small for this reaction, as had been seen earlier in the more approximate 


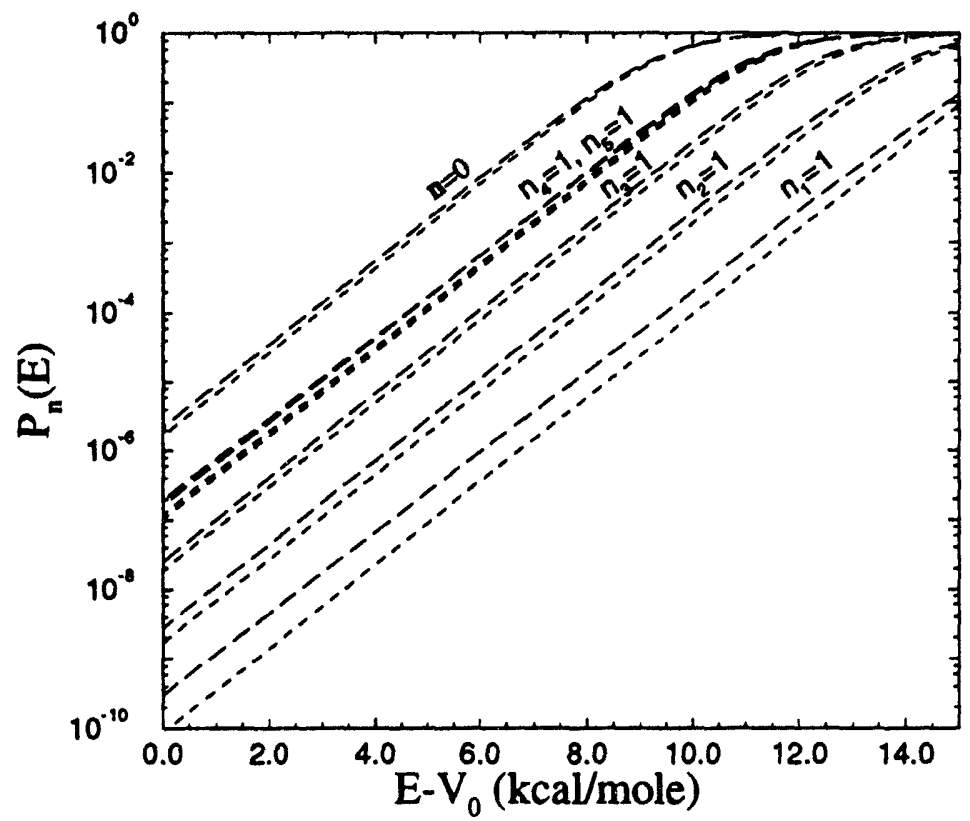

Figure 2.5: Transition state transmission (tunneling) probabilities as a function of energy $\left(E-V_{0}\right)$. Long-dashed curves are the fully anharmonic SCTST and shortdashed curves are the harmonic SCTST results. The top pair of curves is for the ground state $(n=0)$ of the activated complex, and the remaining pairs correspond to its "fundamentals", i.e. one vibrational quantum in each of the 5 modes (the lowest pair corresponding to the highest frequency mode $k=1$, and successively higher ones for $k=2, \ldots, 5)$. 


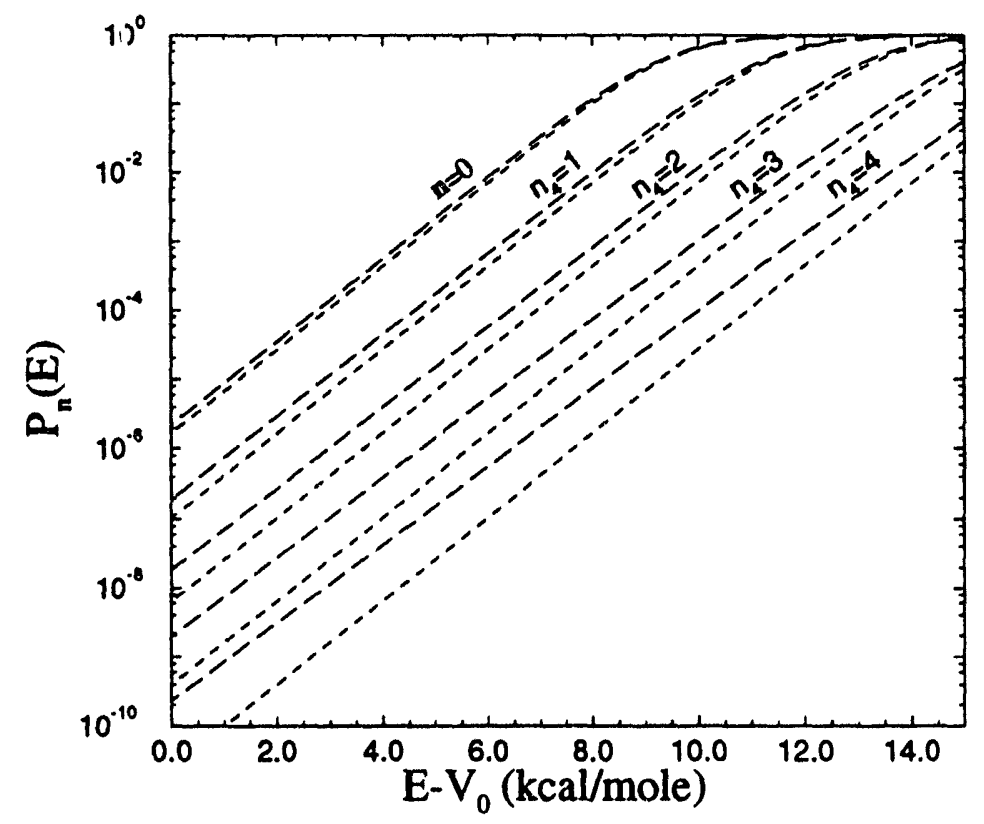

Figure 2.6: Same as Fig. 2.5. The top pair of curves is for the ground state of the activated complex, and successively lower ones are for 1, 2, 3 and 4 quanta in mode $k=4$, i.e. the "fundamental" and the first three "overtones" of this mode.

treatment. ${ }^{131}$ Figure 2.6 shows that the corrections can become significant as more energy is placed into the activated complex, particularly for the low frequency mode 4 that has a large coupling to the reaction coordinate $\left(\bar{x}_{f F}=-28.2 \mathrm{~cm}^{-1}\right)$.

The transmission probabilities have also been obtained including anharmonicity only in the reaction coordinate mode, i.e. setting $x_{k l}=x_{k f}=0$ for all $k$ and $l$ not equal to $F$. The anharmonicity along the reaction coordinate reduces to:

$$
x_{F F}=\frac{\hbar^{2}}{16 \bar{\omega}_{F}^{2}}\left(-f_{F F F}-\frac{5}{3} \frac{f_{F F F}^{2}}{\bar{\omega}_{F}^{2}}\right) .
$$

This is a commonly used approximation ${ }^{133}$ and it corresponds to an anharmonic one-dimensional barrier uncoupled to a harmonic activated complex. Although not shown, the results under this approximation actually gave worse agreement with the fully anharmonic results than the completely harmonic approximation. This is becauce anharmonicity of the one-dimensional barrier thickens it and thus decreases the transmission probability, while coupling of the reaction coordinate to modes of the activated complex (reaction path curvature) typically increases the transmission 
probability to more than off-set the effect of the one-dimensional barrier anharmonicity.

\subsection{Concluding Remarks}

In this chapter, a semiclassical transition state theory has been developed which can be useful in obtaining averaged dynamical quantities for chemical reactions of large systems, be they unimolecular dissociation or bimolecular reaction. The only information about the chemical system which is required is the determination of the local potential energy surface in the vicinity of the transition state, i.e., higher order derivatives. These can be obtained either through direct $a b$ initio techniques or by differentiation of known or supposed potential energy surfaces.

Although not discussed explicitly in the examples of this chapter, thermal rates have also been computed using the SCTST by numerically integrating the Boltzmann average in Eq. (2.1) with agreement to the exact results similar to that seen for the CRP. ${ }^{100,126}$ In the next chapter, a new expression for the rate is derived which permits a direct calculation and thereby provides a more efficient route to determining thermal rate constants. 


\section{Chapter 3}

\section{Semiclassical Transition State \\ Theory - A New Perspective}

\subsection{Introduction}

The emphasis in the previous chapter was on the approximate calculation of the cumulative reaction probability (CRP). In this chapter, we shift the focus onto the calculation of the thermal rate Eq. (2.1). Formally, this rate is simply the Boltzmann average (or Laplace transform) of the CRP and thus provides equivalent information. However, the thermal rate is typically of more experimental interest and in this chapter we obtain expressions for the SCTST thermal rate which can be computed directly without first computing the CRP.

In particular, a difficulty with the CRP calculation described in the previous chapter is that the inversion will not be analytic for higher order perturbation theory and one must provide a prescription by which to choose the physical root. This was avoided in previous work because satisfactory results were obtained at second order in the perturbation. A more serious difficulty is that the presence of Fermi resonances may destroy quantum number assignments and thereby make it impossible to perform the inversion. In previous work ${ }^{104}$ and in recent work by Cohen et al. ${ }^{126}$ this problem has been treated by diagonalization of the resonance polyads at $\theta=0$ with subsequent use of these eigenvalues providing the approximate transmission probabilities. This 
procedure is tantamount to assuming that the couplings between the reactive mode to each of the Fermi resonating states in a given polyad are approximately the same; but in general, this will not be true. The method to be described will obviate both of these difficulties.

Thus the aim of this chapter is to show that the SCTST expression for the thermal rate constant, $k(T)$, can be cast in a much more useful form, one that is simpler to implement than the standard theory and one that can more readily incorporate dynamical extensions to the theory. This new form of the SCTST thermal rate is presented in Sec. 3.2 in addition to presenting an appealing conceptual link to standard TST which will be explored further in Chapter 4. Various aspects of this new rate expression within the context of perturbation theory are illustrated in Sec. 3.3 through the application to representative reactive systems.

\subsection{The "New" Semiclassical Rate Expression}

\subsubsection{A Heuristic Derivation}

The rate expressions described in the previous chapter were constructed with the microcanonical formalism playing the central role. In that discussion, the most relevant quantity is the CRP, and a semiclassical cxpression for its direct evaluation was presented in Eq. (2.14). The computation of the thermal rate then requires a further integration through the use of Eq. (2.1). In this subsection, we present a heuristic argument for a "new" semiclassical rate formula which casts it in a form which can be computed directly. Once the expression is obtained, it is easy to make the argument rigorous, and this will be done in the next subsection.

By substitution of the SCTST CRP, Eq. (2.14), into Eq. (2.1), we obtain the rate formula,

$$
k(T)=\left(2 \pi \hbar Q_{r}\right)^{-1} \mathrm{e}^{-\beta E} \sum_{\underline{n}^{\ddagger}} \int_{\mathcal{E}_{0}}^{\infty} d E\left(1+\mathrm{e}^{2 \theta\left(E, n^{\ddagger}\right)}\right)^{-1},
$$

where the order of summation and integration have been interchanged as is permitted by the theorem ${ }^{134}$ on the integration of infinite series because the terms in the 
summation are uniformly convergent to the CRP. Since one must integrate over all $E$ in Eq. (3.1), it is convenient to change the integration variable from $E$ to $\theta$ and thus avoid having to invert the $E-\theta$ relation. (This analysis assumes that the $E-\theta$ relation is injective and consequently $E(\theta)$ is invertible. The argument is modified somewhat if this is not the case but the form of the result is essentially unchanged as will be presented in the next section.) Thus

$$
\begin{aligned}
\int_{\mathcal{E}_{0}}^{\infty} d E \mathrm{e}^{-\beta E}\left(1+\mathrm{e}^{2 \theta\left(\underline{n}^{\ddagger}, E\right)}\right)^{-1} & =\int_{-\infty}^{\infty} d \theta \frac{\partial E\left(\underline{n}^{\ddagger}, \theta\right)}{\partial \theta} \mathrm{e}^{-\beta E\left(\underline{n}^{\ddagger}, \theta\right)}\left(1+\mathrm{e}^{2 \theta}\right)^{-1} \\
& =\frac{1}{\beta} \int_{-\infty}^{\infty} d \theta \mathrm{e}^{-\beta E\left(\underline{n}^{\ddagger}, \theta\right)} \frac{1}{2} \operatorname{sech}^{2}(\theta)
\end{aligned}
$$

where the last line results from an integration by parts. [The surface terms vanish because $E(\theta \rightarrow \infty)=\mathcal{E}_{0}$, the reaction threshold, and $E(\theta \rightarrow-\infty)=\infty$.] Use of Eq. (3.3) in Eq. (3.1) then gives

$$
k(T)=\left(2 \pi \hbar Q_{r} \beta\right)^{-1} \int_{-\infty}^{\infty} d \theta \frac{1}{2} \operatorname{sech}^{2}(\theta) \sum_{n^{\natural}} \mathrm{e}^{-\beta E\left(\underline{n}^{\natural}, \theta\right)},
$$

where we have again changed the order of summation and integration. This reordering is permitted if the summation, $\sum_{n} \mathrm{e}^{-\beta E\left(n^{\ddagger}, \theta\right)}$ is uniformly convergent. If the Hamiltonian were separable, this condition is satisfied because $E\left(\underset{\sim}{n^{\ddagger}}, \theta\right)$ grows monotonically with the magnitude $\left|n^{\ddagger}\right|$. In the more general non-separable case this latter condition must be satisfied if the semiclassical description is to be correct for the CRP. It should also be noted that this argument does not preclude the possibility that both terms are equal to infinity.

Noting that $(2 \pi \hbar \beta)^{-1}=k T / h$, Eq. (3.4) takes the form of the traditional TST,

$$
k(T)=\frac{k T}{h} \frac{Q^{\ddagger}(T)}{Q_{r}(T)},
$$

by defining the reactive partition function $Q^{\ddagger}(T)$ of the activated complex (including the tunneling correction factor from which it is not separable) as an average of the pre-reactive partition function $Q^{\ddagger}(T, \theta)$ with the weight function $\frac{1}{2} \operatorname{sech}^{2}(\theta)$,

$$
Q^{\ddagger}(T)=\int_{-\infty}^{\infty} d \theta \frac{1}{2} \operatorname{sech}^{2}(\theta) Q^{\ddagger}(T, \theta),
$$


where

$$
Q^{\ddagger}(T, \theta) \equiv \sum_{\underline{n}^{\ddagger}} \mathrm{e}^{-\beta E\left(\underline{n}^{\ddagger}, \theta\right)} .
$$

Equation (3.5) is the basic theoretical result of this chapter. Since $E\left(\underset{\sim}{n^{\ddagger}}, \theta\right)=$ $\mathcal{H}\left(\sim_{\sim}^{\ddagger}, \theta\right)$ is simply the semiclassical Hamiltonian, there is now no need to invert the $E-\theta$ relation (as is necessary to determine the semiclassical CRP.) For applications to date (cf. Secs. 2.4 and 3.3) based on second order perturbation theory, $\mathcal{H}\left(n^{\ddagger}, \theta\right)$ has been a quadratic function of $\theta$ so the analytic inversion has been readily performed, but if one were to use higher order perturbation theory - or even non-perturbative treatments that are necessary when Fermi resonance-like interactions exist - it is a major advantage not to have to obtain $\theta\left(\underset{\sim}{n^{\ddagger}}, E\right)$ explicitly. (Though the quadratic expression is not actually invertible, a practical solution for the inversion obtains by a proper choice of the root - namely the one which agrees with the harmonic result in the limit that the $E-\theta$ relation is linear. When an analytical expression is not available, establishing this connection will certainly be a more arduous task.)

We also note that Eq. (3.5c) is the semiclassical equivalent to

$$
Q^{\ddagger}(T, \theta)=\operatorname{Tr} \mathrm{e}^{-\beta \mathcal{H}(\theta)}
$$

where the quantum mechanical trace is taken over the $(F-1)$ dimensional space spanned by the modes perpendicular to the reaction path. This expression suggests that it is not even necessary to quantize the $(F-1)$ degrees of freedom of the activated complex explicitly but only to determine their partition function as a function of the action, $\theta$, in the reaction coordinate. This opens the door to the possibility of even more accurate ways of determining $Q^{\ddagger}(T, \theta)$ that go beyond the present treatments and which will be explored in Chapter 4.

\subsubsection{A Rigorous Derivation}

Now that the "new" thermal rate expression has been outlined, we present a more rigorous derivation of Eq. (3.5). To this end, we shift the focus even further away from the CRP by returning to an intermediate expression in Miller's derivation within the good action-angle formalism for the semiclassical CRP. ${ }^{41}$ Use of Eq. (2.6) with the 
change of variable suggested by the correspondence relation (2.8), and substitution into the rate expression, Eq. (2.1), readily provides

$$
k(T)=\left(2 \pi \hbar Q_{r}\right)^{-1} \int_{\mathcal{E}_{0}}^{\infty} d E \mathrm{e}^{-\beta E} \int d \theta \sum_{n^{\natural}} \delta\left[E-E\left(\underset{\sim}{n^{\ddagger}}, \theta\right)\right] \frac{\partial E\left(n^{\ddagger}, \theta\right)}{\partial \theta}\left(1+\mathrm{e}^{2 \theta}\right)^{-1}
$$

As described in Sec. 2.2.1, the CRP is obtained by integrating the $\delta$-function over $\theta$. Therein, the existence of only one root of the transcendental equation,

$$
E=E\left(\underline{\sim}^{\ddagger}, \theta\right)
$$

for each set of perpendicular quantum numbers $\underset{\sim}{\ddagger}$ has necessarily assumed that the $E-\theta$ relation is injective. This is certainly the case for a one-dimensional barrier in which the barrier penetration integral is strictly monotonic with the energy [ $c f$. Eq. (2.12)]. However, the generalized barrier penetration integral could conceivably not be so, and thus the possibility that the $E-\theta$ relation is not injective needs to be considered.

Assuming that each $E-\theta$ relation is injective over the energy domain between $\mathcal{E}_{0}$ and $\infty$, we first integrate Eq. (3.7), not over $\theta$ but over $E$, to obtain the rate

$$
k(T)=\left(2 \pi \hbar Q_{a}\right)^{-1} \sum_{n^{\ddagger}} \int_{-\infty}^{\theta_{0, n^{\ddagger}}} d \theta \frac{\partial E\left(n^{\ddagger}, \theta\right)}{\partial \theta}\left(1+\mathrm{e}^{2 \theta}\right)^{-1}
$$

where $\theta_{0, n^{\ddagger}}$ satisfies $E\left(n_{n}^{\ddagger}, \theta_{0, n^{\ddagger}}\right)=\mathcal{E}_{0}$. (Note that as in the derivation of the previous section, the order of summation and integration need be interchanged at several points, and this is permitted here because of analogous arguments.) Integration by parts results in:

$$
k(T)=\left(2 \pi \hbar Q_{a} \beta\right)^{-1}\left[\frac{\mathrm{e}^{-\beta E\left(\underline{n}^{t}, \theta_{0, n^{\natural}}\right)}}{\left(1+\mathrm{e}^{2 \theta}\right)}+\sum_{\underline{n}^{l}} \int_{-\infty}^{\theta_{0, n^{t}}} d \theta \frac{1}{2} \operatorname{sech}^{2}(\theta) \mathrm{e}^{-\beta E\left(\underline{n}^{t}, \theta\right)}\right]
$$

but, $\int_{z}^{\infty} d z^{\prime} \frac{1}{2} \operatorname{sech}^{2}\left(z^{\prime}\right)=\left(1+\mathrm{e}^{2 z}\right)^{-1}$, and so the result may be written as,

$$
k(T)=\left(2 \pi \hbar Q_{a} \beta\right)^{-1} \int_{-\infty}^{\infty} d \theta \sum_{\underline{n}^{\natural}} \frac{1}{2} \operatorname{sech}^{2}(\theta) \mathrm{e}^{-\beta E\left(\underline{n}^{4}, \theta\right)}
$$


where $E\left(\sim_{n}^{\ddagger}, \theta\right)$ is set equal to $\mathcal{E}_{0}$ for $\theta>\theta_{0, n^{\natural}}$. This result reduces to Eq. (3.5) in the limit that each $E-\theta$ relation is injective and that $\theta_{0, n^{l}} \rightarrow \infty$. (In fact, in this limit, the integral over $\theta$ need not be interchanged with the summation over $n^{\ddagger}$ during the derivation.)

More generally, though, each $E-\theta$ relation may be injective only for a reduced range between some $\mathcal{E}_{0, n^{t}}$ and $\infty$. For example, when a perturbation theory is constructed for the Hamiltonian we have seen that the $E-\theta$ relation at second order is not injective. At this order there exist only two branches and it is evident that the second branch - for which $E\left(n^{\ddagger}, \theta \rightarrow \infty\right)=\infty$ - is unphysical and should not be included in the summation. Thus, for each $n_{\sim}^{\ddagger}, E\left(n^{\ddagger}, \theta\right)$ is set equal to $\mathcal{E}_{0, n^{\ddagger}}$, for $\theta>\theta_{0, n^{!}}$, with $\theta_{0, n^{\prime}}$ corresponding to the point at which the two branches meet, i.e.,

$$
\left.\frac{\partial E\left(n^{\ddagger}, \theta\right)}{\partial \theta}\right|_{\theta=\theta_{0, n} !}=0 .
$$

If the perturbation theory were carried out to a higher order, it would be natural to ask how each of the new branches would be treated. By extension from the choice for the second order case, one might argue that all but the last be included, thereby allowing for the possibility of resonant energies. However, it is difficult to determine which branches should be included because they result from the nonseparability of the Hamiltonian and which should be excluded because they are simply an artifact of the mathematics. Hence, in order to obtain a systematic method the energy domain to be used in Eq. (3.11) is taken to be between $\infty$ and the energy at which the first stationary point with respect to $\theta$ is encountered.

\subsubsection{Construction of the Hamiltonian, $\mathcal{H}(\theta)$}

For a general multi-dimensional system the classical Hamiltonian will seldom be integrable, and when it is, it may not be obvious how to obtain the corresponding good global action-angle variables. A standard procedure for avoiding these difficulties is to characterize the local dynamics using perturbation theory in the local region of interest. ${ }^{135}$ Since the rate expression derived in Sec. 3.2 focuses on the transition state region, it is natural to expand the Hamiltonian about the saddle point and use 
perturbation theory to successively obtain an approximate Hamiltonian to an order consistent with the expansion.

As described in Sec. 2.2.2, the semiclassical Hamiltonian may also be obtained from the quantum Hamiltonian. Perturbative methods already developed to describe rovibrational eigenvalue problems ${ }^{107}$, i.e., stationary minima, can be readily adapted to describe the transition state region, i.e., saddle points. The application of canonical Van Vleck perturbation theory (CVPT) ${ }^{136}$ to a rovibrational transition state region was discussed in detail in Chapter 2. In this section, we briefly review the methodology for $J=0$ in order to make a connection with the notation of this chapter.

The vibrational Hamiltonian expanded in mass-weighted normal mode coordinates about the saddle point is:

$$
\mathcal{H}=V_{0}+\frac{1}{2} \sum_{k}^{F}\left(-\hbar^{2} \frac{\partial^{2}}{\partial Q^{2}}+\omega_{k}^{2} Q_{k}^{2}\right)+\frac{1}{6} \sum_{k l m}^{F} f_{k l m} Q_{k} Q_{1} Q_{m}+\frac{1}{24} \sum_{k l m n}^{F} f_{k l m n} Q_{k} Q_{l} Q_{m} Q_{n}
$$

where $V_{0}$ is the potential energy at the saddle point, $\left\{f_{k l m}\right\}\left(\left\{f_{k l m m}\right\}\right)$ are the cubic (quartic) derivatives at the saddle point, and the sums over lowercase letters are unrestricted sums over the $F$ vibrational modes. Recall that the difference from the standard vibrational expansion ${ }^{107}$ is that at zeroth order the potential along the reaction coordinate (mode $F$ ) is a harmonic barrier so that the frequency $\omega_{F}$ is imaginary,

$$
\omega_{F}=i\left|\omega_{r}\right| \equiv i \bar{\omega}_{F}
$$

Using CVPT at second order, the Hamiltonian is given in terms of the "good" quantum numbers by

$$
\mathcal{H}=V_{0}+\sum_{k=1}^{s} \hbar \omega_{k}\left(n_{k}+\frac{1}{2}\right)+\sum_{k \leq k^{\prime}}^{s} \hbar x_{k k^{\prime}}\left(n_{k}+\frac{1}{2}\right)\left(n_{k^{\prime}}+\frac{1}{2}\right),
$$

where the $\omega$ 's and $x$ 's are the "spectroscopic constants" describing the transition state. The resulting $E-\theta$ relation may be written explicitly as

$$
E\left(n^{\ddagger}, \theta\right)=V_{n !}-\hbar \omega_{n}^{*}:\left(\frac{\theta}{\pi}\right)-\hbar x_{F F}\left(\frac{\theta}{\pi}\right)^{2},
$$


where

$$
\begin{aligned}
& V_{n^{\prime}}=V_{0}+\sum_{k=1}^{F-1} \hbar \omega_{k}\left(n_{k}+\frac{1}{2}\right)+\sum_{k \leq k^{\prime}}^{F-1} \hbar x_{k k^{\prime}}\left(n_{k}+\frac{1}{2}\right)\left(n_{k^{\prime}}+\frac{1}{2}\right) \\
& \omega_{n^{\prime}}^{*}=-i \omega_{F}+i \sum_{k=1}^{F-1} x_{k F}\left(n_{k}+\frac{1}{2}\right) .
\end{aligned}
$$

Note that $V_{n !}$ and $\omega_{n !}^{*}$ are real numbers because both the anharmonic coupling $\left\{x_{k r}\right\}$ between the perpendicular and reactive modes and the reactive mode frequency $\omega_{F}$, are imaginary. While only second order perturbation theory has been described explicitly, the generalization to higher order perturbation theory using CVPT $^{120}$ (taking care of the imaginary terms) follows readily.

The thermal rate can now be obtained by substitution of Eq. (3.16) into the expressions developed in Sec. 3.2. However, if there exists a resonance in the expansion at second order, then the corresponding coupling terms cannot be removed perturbatively. ${ }^{107}$ These terms must be retained in the zeroth order Hamiltonian and the remaining coupling terms are successively removed using perturbation theory. The resulting Hamiltonian is block diagonal, characterized by resonance polyads describing each set of resonating states. This Hamiltonian will remain diagonal with respect to $\theta$ because the frequency corresponding to the reactive mode is imaginary and consequently cannot cause a small resonance denominator. Thus the Hamiltonian parametrized by $\theta$ can be diagonalized and use of Eq. (3.5) provides the SCTST thermal rates.

In analogy with the use of diagonalization of the resonance polyads, one can instead retain a large part of the vibrational Hamiltonian at zeroth order, and still be able to use the rate expressions in Sec. 3.2. The only good action that need be identified in the pre-reactive partition function is the action, or quantum number $n_{F}$, associated with the reaction coordinate. Thus one need only use perturbation theory to identify this good action in the Hamiltonian. The remaining off-diagonal couplings can be treated at zeroth order and the pre-reactive partition function calculated using a full Hamiltonian matrix. For large systems in which only a few modes are strongly coupled to the reaction, the weakly coupled modes may still be treated perturbatively thereby reducing the dimensionality of the the pre-reactive partition 
function calculation. The use of these mixed-diagonalization methods is the subject of Chapter 4.

\subsection{Illustrative Examples}

\subsubsection{Eckart barrier}

As a test of the semiclassical expressions presented above, we first consider the one-dimensional Eckart barrier, Eq. (2.37), for which $\mathcal{H}(\theta)$ can be obtained exactly. Evaluation of the barrier penetration integral, ${ }^{110}$

$$
\theta(E)=\int d x \sqrt{2 m[V(x)-E] / \hbar^{2}}
$$

leads to

$$
\theta(E)=\sqrt{\frac{2 m}{\hbar^{2}}} a\left(\sqrt{V_{0}}-\sqrt{E}\right),
$$

for $0 \leq E<V_{0}$. The restriction for $E<V_{0}$ is removed by appealing to the uniformly convergent semiclassical theory. ${ }^{137}$ Inversion of Eq. (3.20) results in a Hamiltonian $E(\theta)$ which is identical in form to that obtained by perturbation theory, Eq. (3.16), except that here the expression is exact. This system thus provides a direct test of the semiclassical approximation involved in the SCTST theory.

The pre-reactive partition function for the Eckart barrier is

$$
Q^{\ddagger}(T, \theta)=\mathrm{e}^{-\beta\left[V_{0}-\hbar \omega^{*}(\theta / \pi)-\hbar x^{*}(\theta / \pi)^{2}\right]},
$$

where

$$
\begin{aligned}
\omega^{*} & =\left(\frac{2 V_{0}}{m a^{2}}\right)^{\frac{1}{2}} \\
x^{*} & =-\frac{\hbar}{2 m a^{2}} .
\end{aligned}
$$

The integral over $\theta$ in Eq. (3.5b) then gives

$$
k(T)=\Gamma^{*} \frac{k T}{h} \frac{e^{-\beta V_{0}}}{Q_{r}}
$$


where the tunneling correction factor $\Gamma^{*}$ is

$$
\Gamma^{*}=\sum_{n=0}^{\infty}\left(\sum_{m=0}^{n} \frac{\left(\beta \hbar \omega^{*}\right)^{2 m}\left(\beta \hbar x^{*}\right)^{n-m}}{(2 m) !(n-m) !}\right) b_{n},
$$

with

$$
b_{n} \equiv(-1)^{n+1}\left(1-2^{1-2 n}\right) B_{2 n},
$$

and where $B_{2 n}$ are Bernoulli Numbers.

In the harmonic limit $x^{*} \rightarrow 0$, the tunneling correction factor reduces to,

$$
\Gamma_{\mathrm{hb}}^{*}=\sum_{n=0}^{\infty}\left(\frac{\left(\beta \hbar \omega^{*}\right)^{2 n}}{(2 n) !}\right) b_{n}
$$

Using the equality, (which can be shown using generating functions ${ }^{138}$ )

$$
\sum_{m=0}^{N} 2^{M}\left(\begin{array}{l}
N \\
m
\end{array}\right) B_{m}=-2\left(2^{N-1}-1\right) B_{n},
$$

Eq. (3.27) can be shown to be equal to the standard result: ${ }^{45}$

$$
\Gamma_{\mathrm{hb}}^{*}=\frac{\hbar \omega \beta / 2}{\sin (\hbar \omega \beta / 2)}
$$

While it is satisfying that the harmonic result is reproduced, it is disappointing that it also reproduces the divergence of the rate found in the standard result for $\beta<$ $2 \pi$. This is not surprising given that the expressions are formally equivalent. The primary advantage of the new expression, however, is that it allows for the inclusion of anharmonicity, which will often extend the limit of convergence beyond that of the harmonic result.

To illustrate the level of accuracy that can be expected of SCTST, Fig. 3.1 presents the SCTST result for $\Gamma^{*}$ as a function of temperature given by Eq. (3.25), compared to the exact quantum results reported by Johnston ${ }^{139}$ for various ratios of $\left|x^{*} / \omega^{*}\right|$. The accuracy of the SCTST result is extremely good up to anharmonicities $\left|x^{*} / \omega^{*}\right|$ of $\sim 20 \%(\simeq \pi / 16)$, and only about a factor of 2 too small by the time it has increased to $\sim 80 \%(\simeq \pi / 4)$. Therfore for systems of chemical interest it is reasonable to expect that the rate expressions in Sec. 3.2 will provide good estimates of the thermal rates in a wide thermal range. 


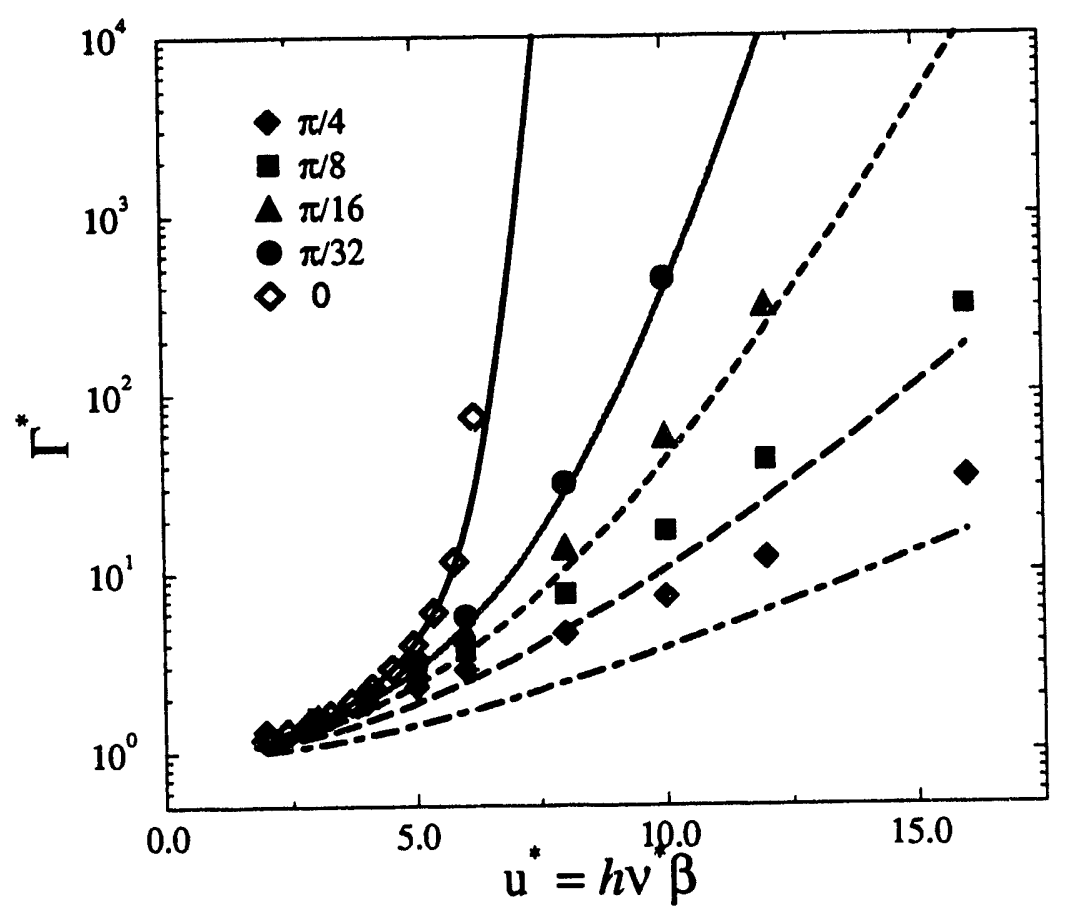

Figure 3.1: The tunneling correction factor $\Gamma^{*}$ for the transmission through Eckart barriers for various relative anharmonicities $\left|x^{*} / \omega^{*}\right|$. Exact results ${ }^{139}$ (points) are compared to the SCTST result (curves). The dotted curve (filled circles) corresponds to $\left|x^{*} / \omega^{*}\right|=\pi / 32$. The short-dashed curve (filled triangles) corresponds to $\left|x^{*} / \omega^{*}\right|=$ $\pi / 16$. The long-dashed curve (filled squares) corresponds to $\left|x^{*} / \omega^{*}\right|=\pi / 8$. The dotdashed curve (filled diamonds) corresponds to $\left|x^{*} / \omega^{*}\right|=\pi / 4$. The solid curve (open diamonds) corresponds to $x^{*} / \omega^{*}=0$ - i.e., the harmonic limit - which diverges at $\beta=2 \pi$. 


\subsubsection{Full-dimensional $\mathrm{H}+\mathrm{H}_{2} \rightarrow \mathrm{H}_{2}+\mathrm{H}$}

The transmission probabilities and thermal rates for the distinguishable $\mathrm{H}+\mathrm{H}_{2}$ reaction with zero angular momentum have recently been obtained using the SCTST discussed in Sec. 2.2.2. ${ }^{104}$ For this reaction, $\mathcal{H}\left(n_{\sim}^{\ddagger}, \theta\right)$ is constructed using the perturbation theory described in Sec. 3.2.3. The cubic and quartic derivatives at the saddle point were obtained by finite differences of the $\mathrm{DMBE}^{127}$ (direct manybody expansion) surface and these can be transformed to provide the "spectroscopic constants" characterizing the transition state region. ${ }^{104}$ Since the out-of-plane degenerate bends are in Fermi resonance with the symmetric stretch, it is necessary to use the degenerate form of perturbation theory. The perturbative Hamiltonian, $\mathcal{H}(\theta)$, is block diagonal with each block labeled by the polyad quantum number of the degenerate modes. In the calculation of the CRP the required inversion of the $E-\theta$ relations must be performed numerically since one must diagonalize each of these blocks as a function of $\theta$. (In previous work ${ }^{104}$ this inversion was approximated using a quadratic form determined by the eigenvalues of the Hamiltonian at $\theta=0$.) However, the $\theta$-dependent anharmonic coupling may be easily included without requiring this inversion by using the SCTST thermal rate expression developed in Sec. 3.2.

To obtain the thermal rate the reactant partition function, $Q_{r}$, must also be obtained. If one ignores ortho-para spin statistics, the partition function can be written as, ${ }^{140}$

$$
Q_{r}=\frac{1}{\sigma}\left(\frac{2 \pi \mu}{h^{2} \beta}\right)^{3 / 2} \sum_{v, J, K} \mathrm{e}^{-\beta E_{v, J, K}},
$$

where $E_{v, J, K}$ aie the rovibrational levels of $\mathrm{H}+\mathrm{H}_{2}$ and $\sigma=2$ is the symmetry number for the homonuclear $\mathrm{H}_{2}$ molecule. The usual practice, however, has been to associate the factor $\sigma$ with the number of reactive pathways and include it within the CRP calculation. ${ }^{49}$ The rovibrational energy levels for $\mathrm{H}_{2}$ were obtained by an exact diagonalization on the asymptotic DMBE surface by Chatfield et al. ${ }^{1}$ and here we use their values. ${ }^{141}$ This insures that any differences between the calculated rate constants using SCTST and the exact results ${ }^{1}$ are due to the approximate calculation of the reactive partition function. For the aid of future studies, the reactant partition functions - not including $\sigma$ - calculated at various sample temperatures for the 


\begin{tabular}{rccccc}
\hline \hline$T(\mathrm{~K})$ & $1 /\left(h Q_{r}\right)$ & $k^{\mathrm{h}}$ & $k^{\mathrm{a}}$ & $k^{\mathrm{ar}}$ & $k^{\mathrm{ex}}$ \\
\hline 200 & $1.12 \times 10^{16}$ & $\infty$ & $8.91 \times 10^{-20}$ & $9.23 \times 10^{-20}$ & $6.428 \times 10^{-20}$ \\
300 & $2.36 \times 10^{13}$ & $\infty$ & $12.1 \times 10^{-18}$ & $12.4 \times 10^{-18}$ & $8.505 \times 10^{-18}$ \\
400 & $8.73 \times 10^{11}$ & $1.51 \times 10^{-16}$ & $1.65 \times 10^{-16}$ & $1.68 \times 10^{-16}$ & $1.291 \times 10^{-16}$ \\
600 & $2.41 \times 10^{10}$ & $1.11 \times 10^{-15}$ & $2.29 \times 10^{-15}$ & $2.38 \times 10^{-15}$ & $1.988 \times 10^{-15}$ \\
700 & $7.82 \times 10^{9}$ & $2.21 \times 10^{-15}$ & $4.80 \times 10^{-15}$ & $5.07 \times 10^{-15}$ & $4.250 \times 10^{-15}$ \\
1000 & $8.47 \times 10^{8}$ & $.79 \times 10^{-14}$ & $1.76 \times 10^{-14}$ & $2.00 \times 10^{-14}$ & $1.578 \times 10^{-14}$ \\
\hline \hline
\end{tabular}

Table 3.1: The $J=0$ reactant partition function and thermal rate constants for the $\mathrm{H}+\mathrm{H}_{2}$ reaction. The inverse of the reactant partition function, $1 /\left(h Q_{r}\right)$, is presented in units of $\mathrm{J}^{-1} \mathrm{~cm}^{3} \mathrm{~s}^{-1}$, and the thermal rates are presented in units of $\mathrm{cm}^{3} \mathrm{molec}^{-1} \mathrm{~s}^{-1}$. $k^{\mathrm{h}}$ is the harmonic SCTST rate. $k^{\mathrm{a}}\left(k^{\mathrm{ar}}\right)$ is the anharmonic SCTST rate including (removing) the Fermi resonance. $k^{\text {ex }}$ is the exact thermal rate of Chatfield et al. ${ }^{1}$

$\mathrm{H}+\mathrm{H}_{2}$ system are presented in Table 3.1 .

In Fig. 3.2, the semiclassical anharmonic thermal rate for the $\mathrm{H}+\mathrm{H}_{2}$ reaction is compared both to the harmonic rate and to the exact rate ${ }^{1}$ on the DMBE surface. Semiclassical rates at various sample temperatures within the range of the figure are also presented in Table 3.1. The anharmonic SCTST results compare well with the exact values, and they converge well below the harmonic linit of $\hbar \omega^{*} \beta=2 \pi$, $T=323 \mathrm{~K}$ for this system. As seen in the CRP results of Sec. 2.4.3, the removal of the Fermi resonance provides poorer results than those seen when it is included; this is in disagreement with calculations in which the removal of the Fermi resonance is treated only approximately. ${ }^{104,126}$

The pre-reactive partition functions at various sample temperatures are shown in Fig. 3.3. As can be seen, they peak in the positive $\theta$ region and broaden with increasing temperature. The broadening results from the increasing importance of non-tunneling transmission, while the peaking in this system results from the strong anharmonicity. The integrands of the semiclassical reactive partition function in Eq. (3.5b) are also shown and they exhibit even stronger peaking around $\theta=0$. The peaking manifests itself in the convergence of the rate, while the asymmetry in the integrand results from tunneling effects. As the temperature increases, tunneling is less significant, and the peaks in the integrand are narrower and more symmetric. 


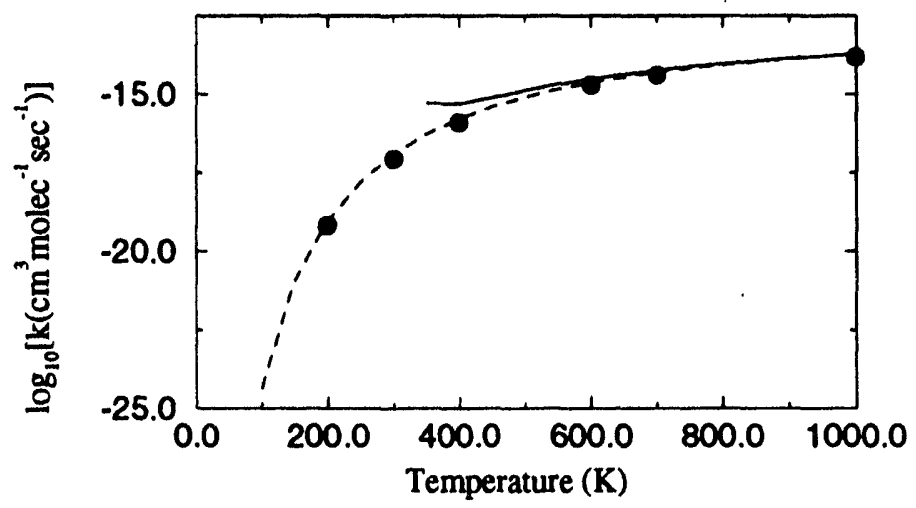

Figure 3.2: The thermal rate for the $\mathrm{H}+\mathrm{H}_{2}$ reaction on the DMBE surface. The standard harmonic result (solid) and the anharmonic semiclassical rate (dashed) are compared to the exact results of Chatfield et al. ${ }^{1}$ (points).

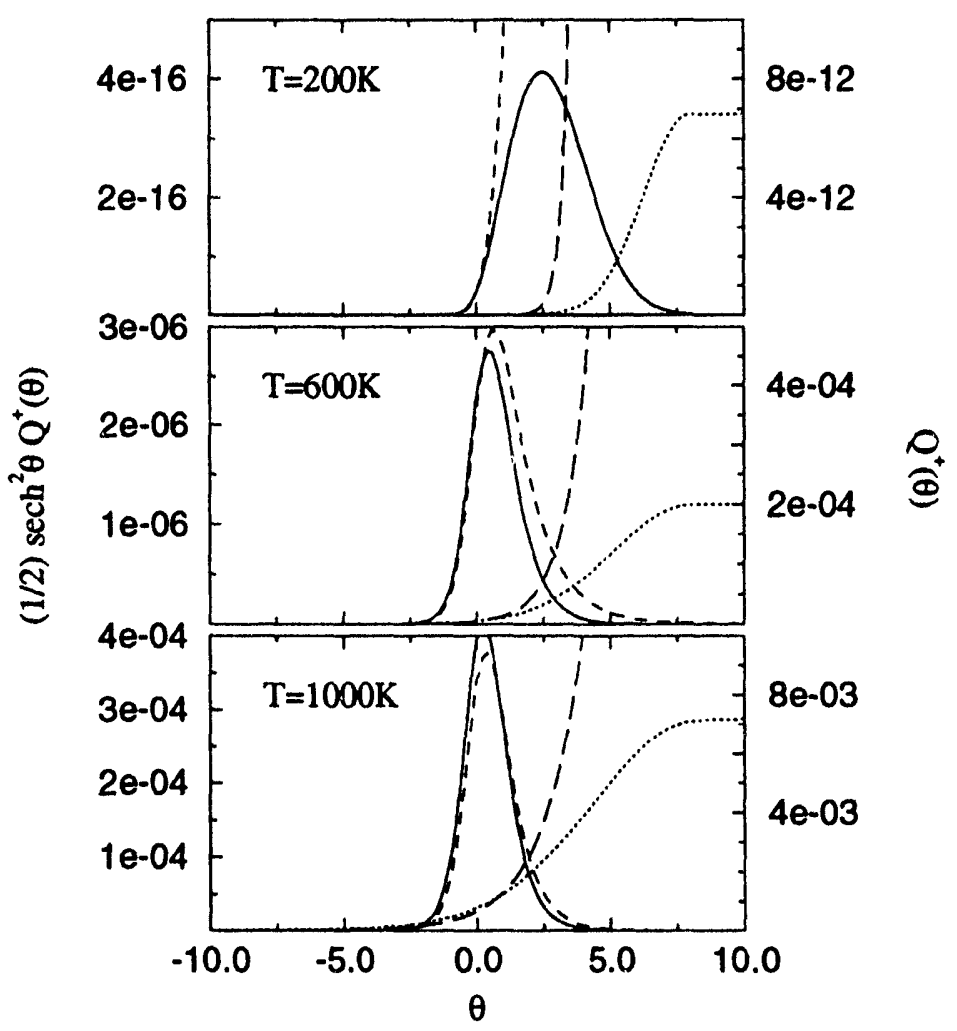

Figure 3.3: SCTST results for the $\mathrm{H}+\mathrm{H}_{2}$ reaction at various temperatures. The long-dashed (dotted) curves are the harmonic (anharmonic) pre-reactive partition functions $Q^{\ddagger}(\theta)$ with the scaling determined by the axes on the righ. The shortdashed (solid) curves are the harmonic (anharmonic) integrands of Eq. (3.5b) with the scaling determined by the axes on the left. 


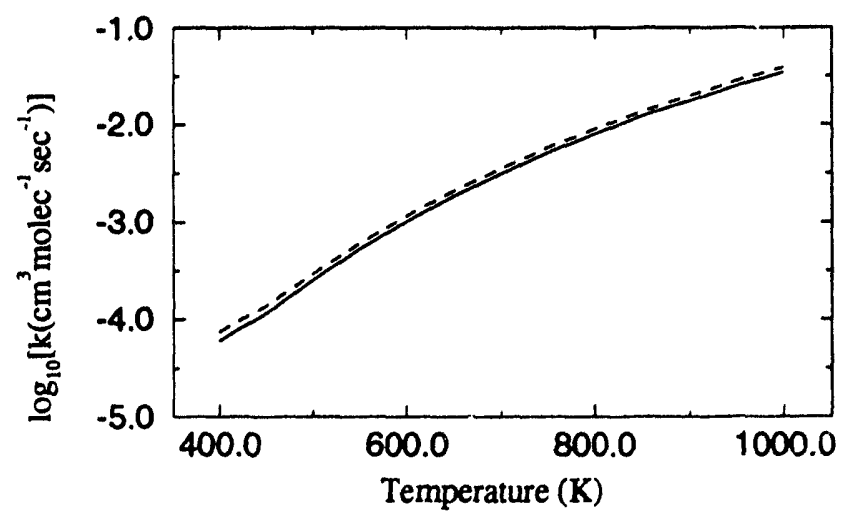

Figure 3.4: The reactive partition function for the formaldehyde dissociation for the harmonic case (solid) is compared to the anharmonic case (dashed).

\subsection{3 $\mathrm{D}_{2} \mathrm{CO} \rightarrow \mathrm{D}_{2}+\mathrm{CO}$}

At temperatures for which there is a non-negligible rate for the dissociation of formaldehyde $\mathrm{D}_{2} \mathrm{CO}$, the dynamics take place on the excited potential energy surfaces $T_{1}$ and $S_{1}$, in addition to the lowest lying surface $S_{0} \cdot{ }^{142}$ Since the theory outlined in Sec. 3.2 does not account for multiple potential energy surfaces nor for nonadiabatic effects, here we focus on the calculation of the reactive partition function, making the non-physical assumption that the dynamics occurs exclusively on the $S_{0}$ surface. Nonetheless the application to this system illustrates the feasibility of the method for unimolecular dissociation on adiabatic surfaces.

The "spectroscopic constants" for the $\mathrm{D}_{2} \mathrm{CO}$ dissociation have been determined using the derivatives of the surface calculated by second-order Moller-Plesset perturbation theory (MP2/DZP) ${ }^{103}$ The reactive partition function, Eq. (3.5b), can be readily obtained and is shown in Fig. 3.4. As has already been noted, ${ }^{131}$ the anharmonic effect on the reactive partition function is small. Unfortunately, the anharmonicity of the reactive mode is also small and, at temperatures below the harmonic cut-off $-400 \mathrm{~K}$ - is not sufficient to ensure the convergence seen in the $\mathrm{H}+\mathrm{H}_{2}$ reaction.

In Fig. 3.5, the pre-reactive partition function, and the integrand for the semiclassical rate [see, Eq. (3.5)] are shown. At the temperatures presented, the thermal rate converges although it is clear that the tail of the integrand is rising with 


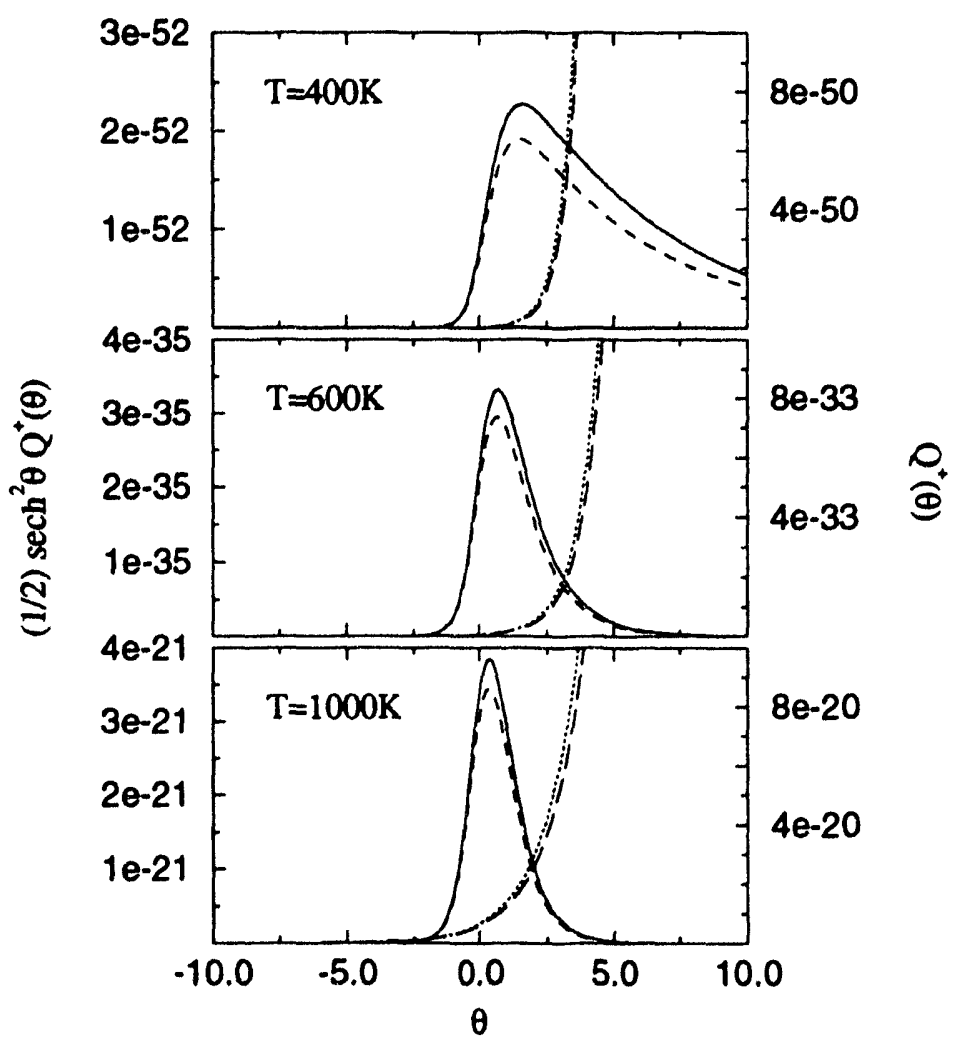

Figure 3.5: SCTST results for the $\mathrm{D}_{2} \mathrm{CO}$ dissociation. The curves are as in Fig. 3.3. 
decreasing temperatures; at still lower temperatures, this tail is not damped and results in the diverging integral. The use of higher order perturbation theory could provide stronger damping terms, and result in convergence at these lower temperatures. This conjecture has yet to be explored.

\subsection{Concluding Remarks}

The primary theoretical development of this chapter has been the determination of a new form for the semiclassical rate expression allowing for the inclusion of anharmonicity (either through perturbation theory or mixed-diagonalization) using a direct analysis of the local Hamiltonian in the transition state region. The prereactive partition function, defined in the chapter, is readily computed in analogy to the calculation of standard partition functions and offers a new tool for the analysis of the convergence of the thermal rate. Moreover, anharmonic effects can actually lead to convergence at low temperatures where the harmonic rate diverges.

These conclusions have been illustrated for the $\mathrm{H}+\mathrm{H}_{2}$ reaction where the strong anharmonicity of the transition state converges the rate at low temperatures. The presence of a Fermi resonance between some of the modes did not present a difficulty because the new thermal rate expression focuses directly on the eigenvalues of the $\theta$-labeled Hamiltonian.

In the formaldehyde dissociation, at second order in perturbation theory, the anharmonic semiclassical rate expression gave a small correction to the harmonic result; but it did not extend the region of convergence. This latter failing is attributed to the small anharmonic coupling of the reaction coordinate at this order in perturbation theory. It is possible that this result can be improved by using still higher order perturbation theory or mixed-diagonalization; the latter approach will be explored in the subsequent chapter. 


\section{Chapter 4}

\section{Semiclassical Transition State Theory - Perturbation Theory Revisited}

\subsection{Introduction}

In the previous chapter a semiclassical rate formula was obtained whose only required input is a Hamiltonian $\mathcal{H}(\theta)$ parametrized by the generalized barrier penetration integral. Following the work in Chapter 2 in which perturbation theory was used to construct a diagonal Hamiltonian of this form - but also labeled by the states of the activated complex - the thermal rate was calculated for several systems. However, if there exists a Fermi resonance, the perturbative Hamiltonian is no longer diagonal; in fact it is block diagonal with each block labeled by a given polyad quantum number. This suggests that, in general, one could treat all the perpendicular modes as if they were in resonance, and obtain a thermal rate constant by diagonalizing the Hamiltonian in the $F-1$ vibrational degrees of freedom. To this aim, the perturbation theory expressions for a vibrational Hamiltonian in which only some degrees of freedom are treated perturbatively and the remaining modes are not, are derived in Sec. 4.3. Because the solution of this Hamiltonian subsequently requires the diagonalization of the reduced degree-of-freedom Hamiltonian, it is referred to 
as mixed-diagonalization.

The least trivial case in which these formulae will apply is two-dimensional as there must be one degree of freedom corresponding to the reaction path - which is treated perturbatively - and at least one additional vibrational perpendicular motion for which the Hamiltonian will be diagonalized. The use of the method is thus illustrated by the collinear $\mathrm{H}+\mathrm{H}_{2}$ reaction in Sec. 4.4.

\subsection{CVPT for Vibrational Hamiltonians}

The standard vibrational perturbation theory Hamiltonian can most easily be obtained using contact transformations; ${ }^{107}$ the method is known by several names, but perhaps the most commonly used term in chemistry is canonical Van Vleck perturbation theory (CVPT). ${ }^{108,109}$ In this section, the derivation of the perturbative Hamiltonian is outlined in order to make connection with the derivation of the results of mixed-diagonalization which are to follow.

The vibrational normal mode Hamiltonian may be written as:

$$
\mathcal{H}=\mathcal{H}_{20}+\mathcal{H}_{30}+\mathcal{H}_{40}+\cdots
$$

where

$$
\begin{aligned}
& \mathcal{H}_{20}=\frac{1}{2} \sum_{k} \omega_{k}\left(p_{k}^{2}+q_{k}^{2}\right) \\
& \mathcal{H}_{30}=\frac{1}{6} \sum_{k, l, m} \kappa_{k l m} q_{k} q_{l} q_{m} \\
& \mathcal{H}_{40}=\frac{1}{24} \sum_{k, l, m, n} \kappa_{k l m n} q_{k} q_{l} q_{m} q_{n} .
\end{aligned}
$$

In the harmonic basis, $\mathcal{H}_{20}$, is diagonal by construction, and the zeroth-order Hamiltonian expanded as a multidimensional Taylor series about a stationary point - e.g. the barrier - is written as:

$$
\mathcal{H}=\mathcal{H}_{0}^{(0)}+\mathcal{H}_{0}^{(1)}+\mathcal{H}_{0}^{(2)}+\cdots
$$

where

$$
\mathcal{H}_{0}^{(n)}=\mathcal{H}_{(n+2) 0}
$$


for each $n$. Using CVPT, one can successively transform this Hamiltonian to a given order, $n$; the $n^{\text {th }}$ order Hamiltonian is also expanded and written as:

$$
\mathcal{H}_{n}=\mathcal{H}_{n}^{(0)}+\mathcal{H}_{n}^{(1)}+\mathcal{H}_{n}^{(2)}+\cdots
$$

The CVPT result for the second order Hamiltonian, for example, is readily found to be

$$
\begin{aligned}
& \mathcal{H}_{2}^{(0)}=\mathcal{H}_{1}^{(0)}=\mathcal{H}_{0}^{(0)} \\
& \mathcal{H}_{2}^{(1)}=\mathcal{H}_{1}^{(1)}=\mathcal{H}_{0}^{(1)}+i\left[S^{(1)}, \mathcal{H}_{0}^{(0)}\right] \\
& \mathcal{H}_{2}^{(2)}=\mathcal{H}_{0}^{(2)}+i\left[S^{(1)}, \mathcal{H}_{0}^{(1)}\right]-\frac{1}{2}\left[S^{(1)},\left[S^{(1)}, \mathcal{H}_{0}^{(0)}\right]+i\left[S^{(2)}, \mathcal{H}_{0}^{(0)}\right]\right.
\end{aligned}
$$

were $S^{(0)}$ and $S^{(1)}$ are the generating functions for zeroth and first order contact transformations, respectively. In the standard approach, the aim of the perturbation expansion is to obtain a $\mathcal{H}_{1}^{(1)}$ which is diagonal and this is accomplished by a prodigious choice of $S^{(1)}$. Since the diagonal matrix elements of odd powers of the position operator in the zeroth order harmonic basis are necessarily zero, then the diagonal matrix elements of $\mathcal{H}_{30}$ are as well, thus,

$$
\mathcal{H}_{2}^{(1)}=\mathcal{H}_{1}^{(1)}=0
$$

which is equivalent to:

$$
\left[S^{(1)}, \mathcal{H}_{0}^{(0)}\right]=i \mathcal{H}_{0}^{(1)}
$$

Since only a second order result is desired, $S^{(2)}$ can be set to zero, and the final expression for the second order Hamiltonian reduces to:

$$
\mathcal{H}_{2}^{(2)}=\mathcal{H}_{0}^{(2)}+i \frac{1}{2}\left[S^{(1)}, \mathcal{H}_{0}^{(1)}\right]
$$

The vibrational expression,

$$
E^{(2)}=\bar{E}_{0}+\sum_{k} \omega_{k}\left(n_{k}+\frac{1}{2}\right)+\sum_{k \leq l} x_{k l}\left(n_{k}+\frac{1}{2}\right)\left(n_{l}+\frac{1}{2}\right)
$$

results by taking the diagonal matrix elements of the second order Hamiltonian, Eq. (4.12). 


\subsection{Mixed-Diagonalization}

In contrast to the CVPT derivation, in mixed-diagonalization the removal of the full $\mathcal{H}_{1}^{(1)}$ term is not desired. Instead, only those terms in $\mathcal{H}_{1}^{(1)}$ which involve the modes treated perturbatively need be removed. For simplicity in the following sections, only mode $F$ will be treated perturbatively.

There is no loss in generality by doing this special case. The formula obtained converts a Hamiltonian in $F$ degrees of freedom into one which is parametrized by the quantum number of the selected mode, which has been treated perturbatively, and is off-diagonal in the remaining $F-1$ degrees of freedom. By repeated application, this prescription can be used to obtain a Hamiltonian which is diagonal in an arbitrary number of modes, $D$, which are treated perturbatively and off-diagonal in the remaining $F-D$ degrees of freedom.

\subsubsection{Notation}

In order to simplify the ensuing discussion, we first introduce some notation. Each of the vibrational terms in the Hamiltonian will be denoted in by:

$$
\begin{aligned}
\mathcal{H}_{k k} & \equiv \frac{1}{2} \omega_{k}\left(p_{k}^{2}+q_{k}^{2}\right) \\
\mathcal{H}_{k l m} & \equiv \kappa_{k l m} q_{k} q_{l} q_{m} \\
\mathcal{H}_{k l m n} & \equiv \kappa_{k l m n} q_{k} q_{l} q_{m} q_{n}
\end{aligned}
$$

and, we also define the following particular forms of $\mathcal{H}_{k l m}$,

$$
\begin{aligned}
\mathcal{H}_{3 ; k k k} & \equiv \mathcal{H}_{k k k}, \\
\mathcal{H}_{5 ; k k l} & \equiv \mathcal{H}_{k k l} \quad \text { if } k \neq l \\
\mathcal{H}_{8 ; k l m} & \equiv \mathcal{H}_{k l m} \quad \text { if }\left|\epsilon_{k l m}\right|=1
\end{aligned}
$$

where $\epsilon_{k l m}$ is the antisymmetric tensor. The operators, $S_{3 ; k k k}, S_{5 ; k k l}$, and $S_{8 ; k l m}$, are defined by the following requirements,

$$
\begin{aligned}
i\left[S_{3 ; k k k}, \mathcal{H}_{0}^{(0)}\right] & =-\mathcal{H}_{3 ; k k k} \\
i\left[S_{5 ; k k l}, \mathcal{H}_{0}^{(0)}\right] & =-\mathcal{H}_{5 ; k k l}
\end{aligned}
$$




$$
i\left[S_{8 ; k l m}, \mathcal{H}_{0}^{(0)}\right]=-\mathcal{H}_{8 ; k l m}
$$

After some algebra, these terms may be evaluated to yield:

$$
\begin{aligned}
S_{3 ; k k k} & =-\frac{2 \kappa_{k k k}}{\omega_{k}}\left(\frac{1}{3} p_{k}^{3}+\frac{1}{2} q_{k} p_{k} q_{k}\right) \\
S_{5 ; k k l} & =-\frac{\kappa_{k k l}}{\omega_{l}\left(4 \omega_{k}^{2}-\omega_{l}^{2}\right)}\left\{\left(2 \omega_{k}^{2}-\omega_{l}^{2}\right) p_{l} q_{k}^{2}+\omega_{k} \omega_{l} q_{l}\left(p_{k} q_{k}+q_{k} p_{k}\right)+2 \omega_{k}^{2} p_{l} p_{k}^{2}\right\} \\
S_{8 ; k l m} & =\kappa_{k l m}\left(\theta_{k l m} p_{k} q_{l} q_{m}+\theta_{l m k} q_{k} p_{l} q_{m}+\theta_{m k l} q_{k} q_{l} p_{m}-\frac{2 \omega_{k} \omega_{l} \omega_{m}}{\Omega_{k l m}} p_{k} p_{l} p_{m}\right) .
\end{aligned}
$$

where

$$
\begin{aligned}
\theta_{k l m} & =\frac{\omega_{k}\left(\omega_{k}^{2}-\omega_{l}^{2}-\omega_{m}^{2}\right)}{\Omega_{k l m}} \\
\Omega_{k l m} & =\left(\omega_{k}+\omega_{l}+\omega_{m}\right)\left(-\omega_{k}+\omega_{l}+\omega_{m}\right)\left(\omega_{k}-\omega_{l}+\omega_{m}\right)\left(\omega_{k}+\omega_{l}-\omega_{m}\right) .
\end{aligned}
$$

It is not hard to show that upon defining

$$
S_{k l m} \equiv S_{8 ; k l m}
$$

with order of the operators in $S_{8 ; k l m}$ fixed, then,

$$
\begin{aligned}
& S_{5 ; k k l}=S_{k k l}, \\
& S_{3 ; k k k}=S_{k k k},
\end{aligned}
$$

and consequently the single form, $S_{k l m}$, suffices to do all calculations. In actual calculations, however, it is convenient to use the particular forms.

It is also useful to define several summation conventions. A single prime on a sum corresponds to the exclusion of mode $F$ for each of the summation variables under the summation symbol, e.g.,

$$
\sum_{k, l}^{\prime} \equiv \sum_{k=1}^{F-1} \sum_{l=1}^{F-1} .
$$

A double prime on a sum excludes an additional mode from the summation and this will be identified in the rare cases that it is used. Variables under the summation which are set apart by parentheses exclude equality, e.g.,

$$
\sum_{m,(k, l)}^{\prime} \equiv \sum_{m=1}^{F-1} \sum_{k \neq l}^{F-1} .
$$




\subsubsection{Obtaining the Generator}

Now, suppose that we wish to do perturbation theory only on a single chosen mode; call this special mode $F$. The form of the Hamiltonian which we want to obtain, should then be:

$$
\begin{aligned}
\overline{\mathcal{H}}_{n_{F}}= & \sum_{k}^{\prime} \mathcal{H}_{k k}+\frac{1}{6} \sum_{k, l, m}^{\prime} \mathcal{H}_{k l m}+\frac{1}{24} \sum_{k, l, m, n}^{\prime} \mathcal{H}_{k l m n} \\
& +\left(\text { operators in } \vec{q} \text { and } \vec{p} \text { with coefficients depending on } n_{F}\right)
\end{aligned}
$$

where the vector operators $\vec{q}$ and $\vec{p}$ do not include those in mode $F$. In analogy with the derivation in Sec. $4.2, S^{(1)}$ should be chosen such that,

$$
\begin{aligned}
\mathcal{H}_{2}^{(1)}=\mathcal{H}_{1}^{(1)} & =\frac{1}{6} \sum_{k, l, m}^{\prime} \mathcal{H}_{k l m} \\
& =\sum_{k<l<m}^{\prime} \mathcal{H}_{8 ; k l m}+\frac{1}{2} \sum_{(k, l)}^{\prime} \mathcal{H}_{5 ; k k l}+\frac{1}{6} \sum_{k}^{\prime} \mathcal{H}_{3 ; k k k}
\end{aligned}
$$

This is tantamount to requiring that $S^{(1)}$ satisfy:

$$
-i\left[S^{(1)}, \mathcal{H}_{0}^{(0)}\right]=\frac{1}{6} \mathcal{H}_{F F F}+\frac{1}{2} \sum_{k}^{\prime} \mathcal{H}_{F F k}+\frac{1}{2} \sum_{k, l}^{\prime} \mathcal{H}_{F k l}
$$

or, alternatively,

$$
-i\left[S^{(1)}, \mathcal{H}_{0}^{(0)}\right]=\frac{1}{6} \mathcal{H}_{3 ; F F F}+\frac{1}{2} \sum_{k}^{\prime}\left(\mathcal{H}_{5 ; F F k}+\mathcal{H}_{5 ; F k k}\right)+\frac{1}{2} \sum_{(k, l)}^{\prime} \mathcal{H}_{8 ; F k l}
$$

Because of the linearity of this expression, the previous results can be combined to obtain:

$$
S^{(1)}=\frac{1}{6} S_{3 ; F F F}+\frac{1}{2} \sum_{k}^{\prime}\left(S_{5 ; F F F k}+S_{5 ; F k k}\right)+\frac{1}{2} \sum_{(k, l)}^{\prime} S_{8 ; F k l}
$$

\subsubsection{Commutators Involving the Generating Function}

To proceed, we need to evaluate the commutators in Eq. (4.10) which, with the choices already made, reduces to:

$$
\begin{aligned}
\mathcal{H}_{2}^{(2)} & =\mathcal{H}_{0}^{(2)}+i\left[S^{(1)}, \mathcal{H}_{0}^{(1)}\right]-\frac{1}{2}\left[S^{(1)}, i\left(\mathcal{H}_{0}^{(1)}-\mathcal{H}_{1}^{(1)}\right)\right] \\
& =\mathcal{H}_{0}^{(2)}+\frac{i}{2}\left[S^{(1)},\left(\mathcal{H}_{0}^{(1)}+\mathcal{H}_{1}^{(1)}\right)\right]
\end{aligned}
$$




$$
=\mathcal{H}_{0}^{(2)}+\frac{i}{2}\left[S^{(1)},\left\{2 \mathcal{H}_{1}^{(1)}+\frac{1}{6} \mathcal{H}_{3 ; F F F}+\frac{1}{2} \sum_{k}^{\prime}\left(\mathcal{H}_{5 ; F F k}+\mathcal{H}_{5 ; F k k}\right)+\frac{1}{2} \sum_{(k, l)}^{\prime} \mathcal{H}_{8 ; F k l}\right\}\right]
$$

with $S^{(1)}$ from Eq. (4.30). Since in the end we will take matrix elements of the Hamiltonian with respect to the $\left|n_{F}\right\rangle$ harmonic oscillator states, only those terms in Eq. (4.31) which have diagonal contributions in $\left|n_{F}\right\rangle$ need be evaluated.

Evaluating $\left[S^{(1)}, \mathcal{H}_{1}^{(1)}\right]$

We first evaluate the titled commutator. Since $\mathcal{H}_{1}^{(1)}$ has no terms in $F$, the only terms that can contribute along the diagonal correspond to the terms in $S^{(1)}$ which are even in $F$. Thus,

$$
\begin{aligned}
\left\langle n_{F}\left|\left[S^{(1)}, \mathcal{H}_{1}^{(1)}\right]\right| n_{F}\right\rangle & =\left\langle n_{F}\left|\left[\left(\frac{1}{2} \sum_{k}^{\prime} S_{5 ; F F k}\right), \mathcal{H}_{1}^{(1)}\right]\right| n_{F}\right\rangle \\
& =\frac{1}{2} \sum_{k}^{\prime}\left[\left\langle n_{F}\left|S_{5 ; F F k}\right| n_{F}\right\rangle, \mathcal{H}_{1}^{(1)}\right] .
\end{aligned}
$$

Use of Eq. (4.19) with $\mathcal{H}_{1}^{(1)}$ taken from $\mathrm{E} \quad$ ?7), readily provides,

$$
\begin{aligned}
\left\langle n_{F}\left|\left[S^{(1)}, \mathcal{H}_{1}^{(1)}\right]\right| n_{F}\right\rangle & =-\frac{1}{2} ._{k} \quad-\left(n_{F}+\frac{1}{2}\right)\left[p_{k}, \mathcal{H}_{1}^{(1)}\right] \\
& =-\frac{1}{2} \sum_{k}^{\prime} \frac{\kappa_{F F k}}{\omega_{k}}\left(n_{F}+\frac{1}{2}\right)\left\{-i \frac{\partial \mathcal{H}_{1}^{(1)}}{\partial q_{k}}\right\} \\
& =\frac{i}{2} \sum_{k} \frac{\kappa_{F F k}}{\omega_{k}}\left(n_{F}+\frac{1}{2}\right) A_{k}^{(2)},
\end{aligned}
$$

where we have defined the quadratic operator, $A_{k}^{(2)}$, as

$$
A_{k}^{(2)} \equiv\left\{\sum_{l<m}^{\prime \prime} \kappa_{k l m} q_{l} q_{m}+\sum_{l}^{\prime \prime} \kappa_{k k l} q_{k} q_{l}+\frac{1}{2} \sum_{l}^{\prime \prime} \kappa_{k l l} q_{l}^{2}+\frac{1}{2} \kappa_{k k k} q_{k}^{2}\right\}=\frac{1}{2} \sum_{l, m}^{\prime} \kappa_{k l m} q_{l} q_{m}
$$

and the double primes on the sums correspond to the exclusion of $F$ and $k$.

Evaluating $\left[S^{(1)}, \mathcal{H}_{3 ; F F F}\right]$

In calculating the titled commutator of this subsection, we first observe that only terms in $S^{(1)}$ which are odd in $F$ will contribute; as opposed to the previous 
subsection, the reduction in terms is not as dramatic:

$$
\begin{aligned}
\left\langle n_{F}\left|\left[S^{(1)}, \mathcal{H}_{3 ; F F F}\right]\right| n_{F}\right\rangle= & \frac{1}{6}\left\langle n_{F}\left|\left[S_{3 ; F F F}, \mathcal{H}_{3 ; F F F}\right]\right| n_{F}\right\rangle \\
& +\frac{1}{2} \sum_{k}^{\prime}\left\langle n_{F}\left|\left[S_{5 ; F k k}, \mathcal{H}_{3 ; F F F}\right]\right| n_{F}\right\rangle \\
& +\frac{1}{2} \sum_{(k, l)}^{\prime}\left\langle n_{F}\left|\left[S_{8 ; F k l}, \mathcal{H}_{3 ; F F F}\right]\right| n_{F}\right\rangle .
\end{aligned}
$$

The first commutator in Eq. (4.35) is the same as that obtained in the standard CVPT formalism; the result can be found in several texts (e.g. Ref. 143), and is

$$
\frac{1}{6}\left\langle n_{F}\left|\left[S_{3 ; F F F}, \mathcal{H}_{3 ; F F F}\right]\right| n_{F}\right\rangle=\frac{i \kappa_{F F}^{2}}{3 \omega_{F}}\left[\frac{15}{4}\left(n_{F}+\frac{1}{2}\right)^{2}+\frac{7}{16}\right] .
$$

The second commutator in Eq. (4.35) is

$$
\begin{aligned}
\frac{1}{2} & \sum_{k}^{\prime}\left\langle n_{F}\left|\left[S_{5 ; F k k}, \mathcal{H}_{3 ; F F F}\right]\right| n_{F}\right\rangle \\
& =-\frac{1}{2} \sum_{k}^{\prime} \frac{\kappa_{F k k} \kappa_{F F F}}{\omega_{F}\left(4 \omega_{k}^{2}-\omega_{F}^{2}\right)}\left\langle n_{F}\left|\left[\left\{\left(2 \omega_{k}^{2}-\omega_{F}^{2}\right) p_{F} q_{k}^{2}+2 \omega_{k}^{2} p_{F} p_{k}^{2}\right\}, q_{F}^{3}\right]\right| n_{F}\right\rangle \\
& =-\frac{1}{2} \sum_{k}^{\prime} \frac{\kappa_{F k k} \kappa_{F F F}}{\omega_{F}\left(4 \omega_{k}^{2}-\omega_{F}^{2}\right)}\left\{\left(2 \omega_{k}^{2}-\omega_{F}^{2}\right) q_{k}^{2}+2 \omega_{k}^{2} p_{k}^{2}\right\}\left\langle n_{F}\left|\left[p_{F}, q_{F}^{3}\right]\right| n_{F}\right\rangle \\
& =\frac{3 i}{2} \sum_{k}^{\prime} \frac{\kappa_{F k k} \kappa_{F F F}}{\omega_{F}\left(4 \omega_{k}^{2}-\omega_{F}^{2}\right)}\left\{\left(2 \omega_{k}^{2}-\omega_{F}^{2}\right) q_{k}^{2}+2 \omega_{k}^{2} p_{k}^{2}\right\}\left(n_{F}+\frac{1}{2}\right) \\
& =-\frac{3 i}{2} \sum_{k}^{\prime} \kappa_{F k k} \kappa_{F F F}\left(\theta_{F k k} q_{k}^{2}-2 \frac{\omega_{F} \omega_{k}^{2}}{\Omega_{F k k}} p_{k}^{2}\right)\left(n_{F}+\frac{1}{2}\right) .
\end{aligned}
$$

The third commutator in Eq. (4.35) is

$$
\begin{aligned}
\frac{1}{2} & \sum_{(k, l)}^{\prime}\left\langle n_{F}\left|\left[S_{8 ; F k l}, \mathcal{H}_{3 ; F F F}\right]\right| n_{F}\right\rangle \\
& =\frac{1}{2} \sum_{(k, l)}^{\prime} \kappa_{F k l} \kappa_{F F F}\left\langle n_{F}\left|\left[\left(\theta_{F k l} p_{F} q_{k} q_{l}-\frac{2 \omega_{F} \omega_{k} \omega_{l}}{\Omega_{F k l}} p_{F} p_{k} p_{l}\right), q_{F}^{3}\right]\right| n_{F}\right\rangle \\
& =\frac{1}{2} \sum_{(k, l)}^{\prime} \kappa_{F k l} \kappa_{F F F}\left(\theta_{F k l} q_{k} q_{l}-\frac{2 \omega_{F} \omega_{k} \omega_{l}}{\Omega_{F k l}} p_{k} p_{l}\right)\left\langle n_{F}\left|\left[p_{F}, q_{F}^{3}\right]\right| n_{F}\right\rangle \\
& =-\frac{3 i}{2} \sum_{(k, l)}^{\prime} \kappa_{F k l} \kappa_{F F F}\left(\theta_{F k l} q_{k} q_{l}-\frac{2 \omega_{F} \omega_{k} \omega_{l}}{\Omega_{F k l}} p_{k} p_{l}\right)\left(n_{F}+\frac{1}{2}\right) .
\end{aligned}
$$

Collecting Eqs. (4.36), (4.37), and (4.38), we obtain:

$$
\left\langle n_{F}\left|\left[S^{(1)}, \mathcal{H}_{3 ; F F F}\right]\right| n_{F}\right\rangle=\frac{i \kappa_{F F F}^{2}}{3 \omega_{F}}\left[\frac{15}{4}\left(n_{F}+\frac{1}{2}\right)^{2}+\frac{7}{16}\right]
$$




$$
\begin{aligned}
& -\frac{3 i}{2} \sum_{k}^{\prime} \kappa_{F k k} \kappa_{F F F}\left(\theta_{F k k} q_{k}^{2}-2 \frac{\omega_{F} \omega_{k}^{2}}{\Omega_{F k k}} p_{k}^{2}\right)\left(n_{F}+\frac{1}{2}\right) \\
& -\frac{3 i}{2} \sum_{(k, l)}^{\prime} \kappa_{F k l} \kappa_{F F F}\left(\theta_{F k l} q_{k} q_{l}-\frac{2 \omega_{F} \omega_{k} \omega_{l}}{\Omega_{F k l}} p_{k} p_{l}\right)\left(n_{F}+\frac{1}{2}\right)
\end{aligned}
$$

and this reduces to

$$
\begin{aligned}
\left\langle n_{F}\left|\left[S^{(1)}, \mathcal{H}_{3 ; F F F}\right]\right| n_{F}\right\rangle= & \frac{i \kappa_{F F F}^{2}}{3 \omega_{F}}\left[\frac{15}{4}\left(n_{F}+\frac{1}{2}\right)^{2}+\frac{7}{16}\right] \\
& -\frac{3 i}{2} \sum_{k, l}^{\prime} \kappa_{F k l} \kappa_{F F F}\left(\theta_{F k l} q_{k} q_{l}-\frac{2 \omega_{F} \omega_{k} \omega_{l}}{\Omega_{F k l}} p_{k} p_{l}\right)\left(n_{F}+\frac{1}{2}\right)
\end{aligned}
$$

which is the desired result of this subsection.

Evaluating $\left[S^{(1)}, \sum_{k}^{\prime}\left(\mathcal{H}_{5 ; F F k}+\mathcal{H}_{5 ; F k k}\right)\right]$

Although the titled commutator of this subsection in toto does not have the symmetry properties which have been used to simplify the commutators in the previous subsections, it does separate into two such pieces, e.g.,

$$
\left[S^{(1)}, \sum_{k}^{\prime}\left(\mathcal{H}_{5 ; F F k}+\mathcal{H}_{5 ; F k k}\right)\right]=\sum_{k}^{\prime}\left[S^{(1)}, \mathcal{H}_{5 ; F F k}\right]+\sum_{k}^{\prime}\left[S^{(1)}, \mathcal{H}_{5 ; F k k}\right]
$$

and this leads to

$$
\begin{aligned}
{\left[S^{(1)}, \sum_{k}^{\prime}\left(\mathcal{H}_{5 ; F F k}+\mathcal{H}_{5 ; F k k}\right)\right] \sim } & \frac{1}{2} \sum_{k, l}^{\prime}\left[S_{5 ; F F l}, \mathcal{H}_{5 ; F F k}\right]+\frac{1}{6} \sum_{k}^{\prime}\left[S_{3 ; F F F}, \mathcal{H}_{5 ; F k k}\right] \\
& +\frac{1}{2} \sum_{k, l}^{\prime}\left[S_{5 ; F l l}, \mathcal{H}_{5 ; F k k}\right]+\frac{1}{2} \sum_{k,(l, m)}^{\prime}\left[S_{8 ; F l m}, \mathcal{H}_{5 ; F k k}\right]
\end{aligned}
$$

where the $\sim$ symbol serves as a reminder that the equality is satisfied only for diagonal matrix elements on the harmonic oscillator basis.

The first commutator in Eq. (4.42) is

$$
\begin{aligned}
\frac{1}{2} \sum_{k, l}^{\prime}\left\langle n_{F}\left|\left[S_{5 ; F F l}, \mathcal{H}_{5 ; F F}\right]\right| n_{F}\right\rangle= & -\frac{1}{2} \sum_{k, l}^{\prime} \frac{\kappa_{F F k} \kappa_{F F l}}{\omega_{l}\left(4 \omega_{F}^{2}-\omega_{l}^{2}\right)}\left\langle n_{F}\right|\left[\left\{\left(2 \omega_{F}^{2}-\omega_{l}^{2}\right) p_{l} q_{F}^{2}\right.\right. \\
& \left.\left.+\omega_{F} \omega_{l} q_{l}\left(p_{F} q_{F}+q_{F} p_{F}\right)+2 \omega_{F}^{2} p_{l} p_{F}^{2}\right\}, q_{F}^{2} q_{k}\right]\left|n_{F}\right\rangle
\end{aligned}
$$

and now we need to differentiate between cases with and without equality between $k$ and $l$,

$$
\frac{1}{2} \sum_{k, l}^{\prime}\left\langle n_{F}\left|\left[S_{5 ; F F l}, \mathcal{H}_{5 ; F F k}\right]\right| n_{F}\right\rangle=
$$




$$
\begin{aligned}
& -\frac{1}{2} \sum_{(k, l)}^{\prime} \frac{\kappa_{F F k} \kappa_{F F l}}{\omega_{l}\left(4 \omega_{F}^{2}-\omega_{l}^{2}\right)}\left\langle n_{F}\left|\left[\left\{\omega_{F} \omega_{l} q_{l}\left(p_{F} q_{F}+q_{F} p_{F}\right)+2 \omega_{F}^{2} p_{l} p_{F}^{2}\right\}, q_{F}^{2} q_{k}\right]\right| n_{F}\right\rangle \\
& -\frac{1}{2} \sum_{k}^{\prime} \frac{\kappa_{F F k} \kappa_{F F k}}{\omega_{k}\left(4 \omega_{F}^{2}-\omega_{k}^{2}\right)}\left\langle n_{F}\right|\left[\left\{\left(2 \omega_{F}^{2}-\omega_{k}^{2}\right) p_{k} q_{F}^{2}\right.\right. \\
& \left.\left.\quad+\omega_{F} \omega_{k} q_{k}\left(p_{F} q_{F}+q_{F} p_{F}\right)+2 \omega_{F}^{2} p_{k} p_{F}^{2}\right\}, q_{F}^{2} q_{k}\right]\left|n_{F}\right\rangle .
\end{aligned}
$$

To evaluate this last expression, we need to recall the following commutators:

$$
\begin{aligned}
\left\langle n_{F}\left|\left[\left(p_{F} q_{F}+q_{F} p_{F}\right), q_{F}^{2}\right]\right| n_{F}\right\rangle & =-4 i\left(n_{F}+\frac{1}{2}\right) \\
\left\langle n_{F}\left|\left[p_{F}^{2}, q_{F}^{2}\right]\right| n_{F}\right\rangle & =0 \\
\left\langle n_{F}\left|\left[p_{k} q_{F}^{2}, q_{k} q_{F}^{2}\right]\right| n_{F}\right\rangle & =-\frac{3 i}{2}\left\{\left(n_{F}+\frac{1}{2}\right)^{2}+\frac{1}{4}\right\} \\
\left\langle n_{F}\left|\left[p_{k} p_{F}^{2}, q_{k} q_{F}^{2}\right]\right| n_{F}\right\rangle & =-\frac{i}{2}\left\{\left(n_{F}+\frac{1}{2}\right)^{2}-\frac{3}{4}\right\}
\end{aligned}
$$

Thus,

$$
\begin{aligned}
& \frac{1}{2} \sum_{k, l}^{\prime}\left\langle n_{F}\left|\left[S_{5 ; F F l}, \mathcal{H}_{5 ; F F k}\right]\right| n_{F}\right\rangle= \\
& \quad 2 i \sum_{(k, l)}^{\prime} \frac{\kappa_{F F k} \kappa_{F F l} \omega_{F}}{\left(4 \omega_{F}^{2}-\omega_{l}^{2}\right)}\left(n_{F}+\frac{1}{2}\right) q_{k} q_{l}+\frac{i}{2} \sum_{k}^{\prime} \frac{\kappa_{F F k} \kappa_{F F k}}{\omega_{k}\left(4 \omega_{F}^{2}-\omega_{k}^{2}\right)} \\
& \quad \times\left[\frac{3}{2}\left(2 \omega_{F}^{2}-\omega_{k}^{2}\right)\left\{\left(n_{F}+\frac{1}{2}\right)^{2}+\frac{1}{4}\right\}+4 \omega_{F} \omega_{k}\left(n_{F}+\frac{1}{2}\right) q_{k}^{2}+\omega_{F}^{2}\left\{\left(n_{F}+\frac{1}{2}\right)^{2}-\frac{3}{4}\right\}\right],
\end{aligned}
$$

which reduces to

$$
\begin{aligned}
& \frac{1}{2} \sum_{k, l}^{\prime}\left\langle n_{F}\left|\left[S_{5 ; F F l}, \mathcal{H}_{5 ; F F k}\right]\right| n_{F}\right\rangle= \\
& \quad-2 i \sum_{k, l}^{\prime} \kappa_{F F k} \kappa_{F F l} \theta_{F F l}\left(n_{F}+\frac{1}{2}\right) q_{k} q_{l} \\
& \quad+\frac{3 i}{16} \sum_{k}^{\prime} \frac{\kappa_{F F k} \kappa_{F F k}}{\omega_{k}\left(4 \omega_{F}^{2}-\omega_{k}^{2}\right)}\left\{\frac{4}{3}\left(8 \omega_{F}^{2}-3 \omega_{k}^{2}\right)\left(n_{F}+\frac{1}{2}\right)^{2}-\omega_{k}^{2}\right\}
\end{aligned}
$$

which is the desired result for the first commutator.

The second commutator in Eq. (4.42) is

$$
\begin{aligned}
\frac{1}{6} \sum_{k}^{\prime}\left\langle n_{F}\left|\left[S_{3 ; F F F}, \mathcal{H}_{5 ; F k k}\right]\right| n_{F}\right\rangle & =-\frac{1}{3} \sum_{k}^{\prime} \frac{\kappa_{F F F} \kappa_{F k k}}{\omega_{F}}\left\langle n_{F}\left|\left[\left(\frac{1}{3} p_{F}^{3}+\frac{1}{2} q_{F} p_{F} q_{F}\right), q_{F} q_{k}^{2}\right]\right| n_{F}\right\rangle \\
& =\frac{i}{2} \sum_{k}^{\prime} \frac{\kappa_{F F F} \kappa_{F k k}}{\omega_{F}}\left(n_{F}+\frac{1}{2}\right) q_{k}^{2}
\end{aligned}
$$

where we have used the commutators:

$$
\left\langle n_{F}\left|\left[q_{F} p_{F} q_{F}, q_{F}\right]\right| n_{F}\right\rangle=-i\left(n_{F}+\frac{1}{2}\right)
$$




$$
\left\langle n_{F}\left|\left[p_{F}^{3}, q_{F}\right]\right| n_{F}\right\rangle=-3 i\left(n_{F}+\frac{1}{2}\right)
$$

The third commutator in Eq. (4.42) is

$$
\begin{aligned}
\frac{1}{2} \sum_{k, l}^{\prime}\left\langle n_{F}\left|\left[S_{5 ; F l l}, \mathcal{H}_{5 ; F k k}\right]\right| n_{F}\right\rangle= & -\frac{1}{2} \sum_{k, l}^{\prime} \frac{\kappa_{F l l} \kappa_{F k k}}{\omega_{F}\left(4 \omega_{l}^{2}-\omega_{F}^{2}\right)}\left\langle n_{F}\right|\left[\left\{\left(2 \omega_{l}^{2}-\omega_{F}^{2}\right) p_{F} q_{l}^{2}\right.\right. \\
& \left.\left.+\omega_{l} \omega_{F} q_{F}\left(p_{l} q_{l}+q_{l} p_{l}\right)+2 \omega_{l}^{2} p_{F} p_{l}^{2}\right\}, q_{F} q_{k}^{2}\right]\left|n_{F}\right\rangle
\end{aligned}
$$

and, as with Eq. (4.43), we now need to differentiate between cases with and without equality between $k$ and $l$,

$$
\begin{aligned}
& \frac{1}{2} \sum_{k, l}^{\prime}\left\langle n_{F}\left|\left[S_{5 ; F l l}, \mathcal{H}_{5 ; F k k}\right]\right| n_{F}\right\rangle= \\
& \quad-\frac{1}{2} \sum_{(k, l)}^{\prime} \frac{\kappa_{F l l} \kappa_{F k k}}{\omega_{F}\left(4 \omega_{l}^{2}-\omega_{F}^{2}\right)}\left\langle n_{F}\left|\left[\left\{\left(2 \omega_{l}^{2}-\omega_{F}^{2}\right) p_{F} q_{l}^{2}+2 \omega_{l}^{2} p_{F} p_{l}^{2}\right\}, q_{F} q_{k}^{2}\right]\right| n_{F}\right\rangle \\
& \quad-\frac{1}{2} \sum_{k}^{\prime} \frac{\kappa_{F k k} \kappa_{F k k}}{\omega_{F}\left(4 \omega_{k}^{2}-\omega_{F}^{2}\right)}\left\langle n_{F}\right|\left[\left\{\left(2 \omega_{k}^{2}-\omega_{F}^{2}\right) p_{F} q_{k}^{2}\right.\right. \\
& \left.\left.\quad+\omega_{k} \omega_{F} q_{F}\left(p_{k} q_{k}+q_{k} p_{k}\right)+2 \omega_{k}^{2} p_{F} p_{k}^{2}\right\}, q_{F} q_{k}^{2}\right]\left|n_{F}\right\rangle .
\end{aligned}
$$

Making use of the commutators,

$$
\begin{aligned}
\left\langle n_{F}\left|\left[q_{F}\left(p_{k} q_{k}+q_{k} p_{k}\right), q_{F} q_{k}^{2}\right]\right| n_{F}\right\rangle & =-4 i\left(n_{F}+\frac{1}{2}\right) q_{k}^{2} \\
\left\langle n_{F}\left|\left[p_{F} p_{k}^{2}, q_{F} q_{k}^{2}\right]\right| n_{F}\right\rangle & =-\frac{i}{2}\left(p_{k}^{2} q_{k}^{2}+q_{k}^{2} p_{k}^{2}\right)
\end{aligned}
$$

leads to the following simplifications,

$$
\begin{aligned}
\frac{1}{2} \sum_{k, l}^{\prime}\left\langle n_{F}\left|\left[S_{5 ; F l l}, \mathcal{H}_{5 ; F k k}\right]\right| n_{F}\right\rangle= & \frac{i}{2} \sum_{(k, l)}^{\prime} \frac{\kappa_{F l l} \kappa_{F k k}}{\omega_{F}\left(4 \omega_{l}^{2}-\omega_{F}^{2}\right)}\left\{\left(2 \omega_{l}^{2}-\omega_{F}^{2}\right) q_{l}^{2}+2 \omega_{l}^{2} p_{l}^{2}\right\} q_{k}^{2} \\
& +\frac{i}{2} \sum_{k}^{\prime} \frac{\kappa_{F k k} \kappa_{F k k}}{\omega_{F}\left(4 \omega_{k}^{2}-\omega_{F}^{2}\right)}\left\{\left(2 \omega_{k}^{2}-\omega_{F}^{2}\right) q_{k}^{4}\right. \\
& \left.+4 \omega_{k} \omega_{F}\left(n_{F}+\frac{1}{2}\right) q_{k}^{2}+\omega_{k}^{2}\left(p_{k}^{2} q_{k}^{2}+q_{k}^{2} p_{k}^{2}\right)\right\} .
\end{aligned}
$$

The cases can now be combined to yield

$$
\begin{aligned}
\frac{1}{2} \sum_{k, l}^{\prime}\left\langle n_{F}\left|\left[S_{5 ; F l l}, \mathcal{H}_{5 ; F k k}\right]\right| n_{F}\right\rangle= & -\frac{i}{2} \sum_{k, l}^{\prime} \kappa_{F l l} \kappa_{F k k}\left\{\theta_{F l l} q_{k}^{2} q_{l}^{2}-\omega_{F} \omega_{l}^{2} \Omega_{F l l}^{-1}\left(p_{l}^{2} q_{k}^{2}+q_{k}^{2} p_{l}^{2}\right)\right\} \\
& -2 i \sum_{k}^{\prime} \kappa_{F k k} \kappa_{F k k} \theta_{k k F}\left(n_{F}+\frac{1}{2}\right) q_{k}^{2},
\end{aligned}
$$

which is the desired result for the third commutator. 
The fourth commutator in Eq. (4.42) is

$$
\begin{aligned}
& \frac{1}{2} \sum_{k,(l, m)}^{\prime}\left\langle n_{F}\left|\left[S_{8 ; F l m}, \mathcal{H}_{5 ; F k k}\right]\right| n_{F}\right\rangle= \\
& \quad \frac{1}{2} \sum_{k,(l, m)}^{\prime} \kappa_{F l m} \kappa_{F k k}\left\langle n_{F}\right|\left[\left(\theta_{F l m} p_{F} q_{l} q_{m}+\theta_{l m F} q_{F} p_{l} q_{m}+\theta_{m F l} q_{F} q_{l} p_{m}\right.\right. \\
& \left.\left.\quad-\frac{2 \omega_{F} \omega_{l} \omega_{m}}{\Omega_{F l m}} p_{F} p_{l} p_{m}\right), q_{F} q_{k}^{2}\right]\left|n_{F}\right\rangle .
\end{aligned}
$$

It is easy to obtain the following commutators for $l \neq m$ :

$$
\begin{aligned}
\left\langle n_{F}\left|\left[q_{F} p_{l} q_{m}, q_{F} q_{k}^{2}\right]\right| n_{F}\right\rangle & =-2 i\left(n_{F}+\frac{1}{2}\right) q_{m} q_{l} \delta_{l, k} \\
\left\langle n_{F}\left|\left[p_{F} p_{l} p_{m}, q_{F} q_{k}^{2}\right]\right| n_{F}\right\rangle & =-\frac{i}{2}\left(p_{l} p_{m} q_{k}^{2}+q_{k}^{2} p_{l} p_{m}\right)
\end{aligned}
$$

where $\delta$ is the Kroneker delta function. Thus

$$
\begin{aligned}
& \frac{1}{2} \sum_{k,(l, m)}^{\prime}\left\langle n_{F}\left|\left[S_{8 ; F l m}, \mathcal{H}_{5 ; F k k}\right]\right| n_{F}\right\rangle= \\
& \quad-\frac{i}{2} \sum_{k,(l, m)}^{\prime} \kappa_{F l m} \kappa_{F k k}\left\{\theta_{F l m} q_{k}^{2} q_{l} q_{m}-\omega_{F} \omega_{l} \omega_{m} \Omega_{F l m}^{-1}\left(p_{l} p_{m} q_{k}^{2}+q_{k}^{2} p_{l} p_{m}\right)\right\} \\
& \quad-2 i \sum_{l, m}^{\prime} \kappa_{F l m} \kappa_{F l l} \theta_{l m F}\left(n_{F}+\frac{1}{2}\right) q_{l} q_{m},
\end{aligned}
$$

is the desired result for the fourth commutator.

Combining Eqs. (4.42), (4.47), (4.48), (4.54), and (4.57), leads to

$$
\begin{aligned}
\sum_{k}^{\prime} & \left\langle n_{F}\left|\left[S^{(1)},\left(\mathcal{H}_{5 ; F F k}+\mathcal{H}_{5 ; F k k}\right)\right]\right| n_{F}\right\rangle= \\
& -2 i \sum_{k, l}^{\prime} \kappa_{F F k} \kappa_{F F l} \theta_{F F l}\left(n_{F}+\frac{1}{2}\right) q_{k} q_{l} \\
& +\frac{3 i}{16} \sum_{k}^{\prime} \frac{\kappa_{F F k} \kappa_{F F k}}{\omega_{k}\left(4 \omega_{F}^{2}-\omega_{k}^{2}\right)}\left\{\frac{4}{3}\left(8 \omega_{F}^{2}-3 \omega_{k}^{2}\right)\left(n_{F}+\frac{1}{2}\right)^{2}-\omega_{k}^{2}\right\} \\
& +\frac{i}{2} \sum_{k}^{\prime} \frac{\kappa_{F F F} \kappa_{F k k}}{\omega_{F}}\left(n_{F}+\frac{1}{2}\right) q_{k}^{2}-2 i \sum_{k}^{\prime} \kappa_{F k k} \kappa_{F k k} \theta_{k k F}\left(n_{F}+\frac{1}{2}\right) q_{k}^{2} \\
& -\frac{i}{2} \sum_{k, l}^{\prime} \kappa_{F l l} \kappa_{F k k}\left\{\theta_{F l l} q_{k}^{2} q_{l}^{2}-\omega_{F} \omega_{l}^{2} \Omega_{F l l}^{-1}\left(p_{l}^{2} q_{k}^{2}+q_{k}^{2} p_{l}^{2}\right)\right\} \\
& -\frac{i}{2} \sum_{k,(l, m)}^{\prime} \kappa_{F l m} \kappa_{F k k}\left(\theta_{F l m} q_{k}^{2} q_{l} q_{m}-\omega_{F} \omega_{l} \omega_{m} \Omega_{F l m}^{-1}\left(p_{l} p_{m} q_{k}^{2}+q_{k}^{2} p_{l} p_{m}\right)\right) \\
& -2 i \sum_{l, m}^{\prime} \kappa_{F l m} \kappa_{F l l} \theta_{l m F}\left(n_{F}+\frac{1}{2}\right) q_{l} q_{m} .
\end{aligned}
$$

This expression can be simplified to:

$$
\sum_{k}^{\prime}\left\langle n_{F}\left|\left[S^{(1)},\left(\mathcal{H}_{5 ; F F k}+\mathcal{H}_{5 ; F k k}\right)\right]\right| n_{F}\right\rangle=
$$




$$
\begin{aligned}
& +\frac{3 i}{16} \sum_{k}^{\prime} \frac{\kappa_{F F k} \kappa_{F F k}}{\omega_{k}\left(4 \omega_{F}^{2}-\omega_{k}^{2}\right)}\left\{\frac{4}{3}\left(8 \omega_{F}^{2}-3 \omega_{k}^{2}\right)\left(n_{F}+\frac{1}{2}\right)^{2}-\omega_{k}^{2}\right\} \\
& +\frac{i}{2} \sum_{k}^{\prime} \frac{\kappa_{F F F} \kappa_{F k k}}{\omega_{F}}\left(n_{F}+\frac{1}{2}\right) q_{k}^{2} \\
& -2 i \sum_{k, l}^{\prime}\left\{\kappa_{F F k} \kappa_{F F l} \theta_{F F l}+\kappa_{F k l} \kappa_{F k k} \theta_{k l F}\right\}\left(n_{F}+\frac{1}{2}\right) q_{k} q_{l} \\
& -\frac{i}{2} \sum_{k, l, m}^{\prime} \kappa_{F l m} \kappa_{F k k}\left(\theta_{F l m} q_{k}^{2} q_{l} q_{m}-\omega_{F} \omega_{l} \omega_{m} \Omega_{F l m}^{-1}\left(p_{l} p_{m} q_{k}^{2}+q_{k}^{2} p_{l} p_{m}\right)\right),
\end{aligned}
$$

which is the main result of this subsection.

Evaluating $\left[S^{(1)}, \Sigma_{(k, l)}^{\prime} \mathcal{H}_{8 ; F k l}\right]$

In calculating, the titled commutator of this subsection, we observe that it has the same symmetry property as the commutator in Eq. (4.35);

$$
\begin{aligned}
\sum_{(k, l)}^{\prime}\left\langle n_{F}\left|\left[S^{(1)}, \mathcal{H}_{8 ; F k l}\right]\right| n_{F}\right\rangle= & \frac{1}{6} \sum_{(k, l)}^{\prime}\left\langle n_{F}\left|\left[S_{3 ; F F F}, \mathcal{H}_{8 ; F k l}\right]\right| n_{F}\right\rangle \\
& +\frac{1}{2} \sum_{m,(k, l)}^{\prime}\left\langle n_{F}\left|\left[S_{5 ; F m m}, \mathcal{H}_{8 ; F k l}\right]\right| n_{F}\right\rangle \\
& +\frac{1}{2} \sum_{(m, n),(k, l)}^{\prime}\left\langle n_{F}\left|\left[S_{8 ; F m n}, \mathcal{H}_{8 ; F k l}\right]\right| n_{F}\right\rangle .
\end{aligned}
$$

The first of these commutators is similar to Eq. (4.48), and one can readily obtain:

$$
\frac{1}{6} \sum_{(k, l)}^{\prime}\left\langle n_{F}\left|\left[S_{3 ; F F F}, \mathcal{H}_{5 ; F k l}\right]\right| n_{F}\right\rangle=\frac{i}{2} \sum_{(k, l)}^{\prime} \frac{\kappa_{F F F} \kappa_{F k l}}{\omega_{F}}\left(n_{F}+\frac{1}{2}\right) q_{k} q_{l} .
$$

The evaluation of the other commutators is more subtle as one has to consider the non-commutativity of the modes not equal to $F$.

The second commutator in Eq. (4.60) is

$$
\begin{aligned}
& \frac{1}{2} \sum_{m,(k, l)}^{\prime}\left\langle n_{F}\left|\left[S_{5 ; F m m}, \mathcal{H}_{5 ; F k l}\right]\right| n_{F}\right\rangle= \\
& \quad-\frac{1}{2} \sum_{m,(k, l)}^{\prime} \frac{\kappa_{F m m} \kappa_{F k l}}{\omega_{F}\left(4 \omega_{m}^{2}-\omega_{F}^{2}\right)}\left\langle n_{F}\right|\left[\left\{\left(2 \omega_{m}^{2}-\omega_{F}^{2}\right) p_{F} q_{m}^{2}\right.\right. \\
& \left.\left.\quad+\omega_{m} \omega_{F} q_{F}\left(p_{m} q_{m}+q_{m} p_{m}\right)+2 \omega_{m}^{2} p_{F} p_{m}^{2}\right\}, q_{F} q_{k} q_{l}\right]\left|n_{F}\right\rangle
\end{aligned}
$$

and, as with Eq. (4.43), we now need to differentiate between cases with and without equality between $m$ and $k$ or $l$,

$$
\frac{1}{2} \sum_{(k, l, m)}^{\prime}\left\langle n_{F}\left|\left[S_{5 ; F m m}, \mathcal{H}_{5 ; F k l}\right]\right| n_{F}\right\rangle=
$$




$$
\begin{aligned}
& -\frac{1}{2} \sum_{k, l, m}^{\prime} \frac{\kappa_{F m m} \kappa_{F k l}}{\omega_{F}\left(4 \omega_{m}^{2}-\omega_{F}^{2}\right)}\left\langle n_{F}\left|\left[\left\{\left(2 \omega_{m}^{2}-\omega_{F}^{2}\right) p_{F} q_{m}^{2}+2 \omega_{m}^{2} p_{F} p_{m}^{2}\right\}, q_{F} q_{k} q_{l}\right]\right| n_{F}\right\rangle \\
& -\sum_{(k, l)}^{\prime} \frac{\kappa_{F k k} \kappa_{F k l}}{\omega_{F}\left(4 \omega_{k}^{2}-\omega_{F}^{2}\right)}\left\langle n_{F}\right|\left[\left\{\left(2 \omega_{k}^{2}-\omega_{F}^{2}\right) p_{F} q_{k}^{2}+\omega_{k} \omega_{F} q_{F}\left(p_{k} q_{k}+q_{k} p_{k}\right)\right.\right. \\
& \left.\left.+2 \omega_{k}^{2} p_{F} p_{k}^{2}\right\}, q_{F} q_{k} q_{l}\right]\left|n_{F}\right\rangle .
\end{aligned}
$$

Evaluation of the matrix elements in this expression results in:

$$
\begin{aligned}
& \frac{1}{2} \sum_{(k, l, m)}^{\prime}\left\langle n_{F}\left|\left[S_{5 ; F m m}, \mathcal{H}_{5 ; F k l}\right]\right| n_{F}\right\rangle= \\
& \quad \frac{i}{2} \sum_{k, l, m}^{\prime} \frac{\kappa_{F m m} \kappa_{F k l}}{\omega_{F}\left(4 \omega_{m}^{2}-\omega_{F}^{2}\right)}\left\{\left(2 \omega_{m}^{2}-\omega_{F}^{2}\right) q_{m}^{2}+2 \omega_{m}^{2} p_{m}^{2}\right\} q_{k} q_{l} \\
& \quad+i \sum_{(k, l)}^{\prime} \frac{\kappa_{F k k} \kappa_{F k l}}{\omega_{F}\left(4 \omega_{k}^{2}-\omega_{F}^{2}\right)}\left\{\left(2 \omega_{k}^{2}-\omega_{F}^{2}\right) q_{k}^{3} q_{l}+\omega_{k}^{2}\left(q_{l} q_{k} p_{k}^{2}+p_{k}^{2} q_{l} q_{k}\right)\right. \\
& \left.\quad+2 \omega_{k} \omega_{F}\left(n_{F}+\frac{1}{2}\right) q_{k} q_{l}\right\}
\end{aligned}
$$

which further reduces to:

$$
\begin{aligned}
& \frac{1}{2} \sum_{m,(k, l)}^{\prime}\left\langle n_{F}\left|\left[S_{5 ; F m m}, \mathcal{H}_{5 ; F k l}\right]\right| n_{F}\right\rangle= \\
& \quad \frac{i}{2} \sum_{m,(k, l)}^{\prime} \frac{\kappa_{F m m} \kappa_{F k l}}{\omega_{F}\left(4 \omega_{m}^{2}-\omega_{F}^{2}\right)}\left\{\left(2 \omega_{m}^{2}-\omega_{F}^{2}\right) q_{k} q_{l} q_{m}^{2}+\omega_{m}^{2}\left(q_{l} q_{k} p_{k}^{2}+p_{k}^{2} q_{l} q_{k}\right)\right\} \\
& \quad+2 i \sum_{(k, l)}^{\prime} \frac{\kappa_{F k k} \kappa_{F k l} \omega_{k}}{\left(4 \omega_{k}^{2}-\omega_{F}^{2}\right)}\left(n_{F}+\frac{1}{2}\right) q_{k} q_{l} .
\end{aligned}
$$

The third commutator in Eq. (4.60) is

$$
\begin{aligned}
& \frac{1}{2} \sum_{(m, n),(k, l)}^{\prime}\left\langle n_{F}\left|\left[S_{8 ; F m n}, \mathcal{H}_{3 ; F k l}\right]\right| n_{F}\right\rangle= \\
& \quad \frac{1}{2} \sum_{(m, n),(k, l)}^{\prime} \kappa_{F m n} \kappa_{F k l}\left\langle n_{F}\right|\left[\left(\theta_{F m n} p_{F} q_{m} q_{n}+\theta_{m n F} q_{F} p_{m} q_{n}\right.\right. \\
& \left.\left.\quad+\theta_{n F m} q_{F} q_{m} p_{n}-\frac{2 \omega_{F} \omega_{m} \omega_{n}}{\Omega_{F m n}} p_{F} p_{m} p_{n}\right), q_{F} q_{k} q_{l}\right]\left|n_{F}\right\rangle .
\end{aligned}
$$

We first evaluate the following commutators, [with $(m \neq n)$ and $(k \neq l)]$ :

$$
\begin{aligned}
& \left\langle n_{F}\left|\left[p_{F} q_{m} q_{n}, q_{F} q_{k} q_{l}\right]\right| n_{F}\right\rangle=-i q_{m} q_{n} q_{k} q_{l} \\
& \left\langle n_{F}\left|\left[q_{F} p_{m} q_{n}, q_{F} q_{k} q_{l}\right]\right| n_{F}\right\rangle=-i\left(n_{F}+\frac{1}{2}\right) q_{n}\left(q_{l} \delta_{m, k}+q_{k} \delta_{m, l}\right) \\
& \left\langle n_{F}\left|\left[q_{F} q_{m} p_{n}, q_{F} q_{k} q_{l}\right]\right| n_{F}\right\rangle=-i\left(n_{F}+\frac{1}{2}\right) q_{m}\left(q_{l} \delta_{n, k}+q_{k} \delta_{n, l}\right)
\end{aligned}
$$


and

$$
\begin{aligned}
& \left\langle n_{F}\left|\left[p_{F} p_{m} p_{n}, q_{F} q_{k} q_{l}\right]\right| n_{F}\right\rangle= \\
& \begin{cases}-i p_{m} p_{n} q_{k} q_{l}, & \text { if }(m \neq k, l) \text { and }(n \neq k, l) ; \\
-\frac{i}{2} p_{n} q_{l}\left(q_{m} p_{m}+p_{m} q_{m}\right), & \text { î́ }(m=k) \text { and }(n \neq k, l) ; \\
-\frac{i}{2} p_{n} q_{k}\left(q_{m} p_{m}+p_{m} q_{m}\right), & \text { if }(m=l) \text { and }(n \neq k, l) ; \\
-\frac{i}{2} p_{m} q_{l}\left(q_{n} p_{n}+p_{n} q_{n}\right), & \text { if }(m \neq k, l) \text { and }(n=k) ; \\
-\frac{i}{2} p_{m} q_{k}\left(q_{n} p_{n}+p_{n} q_{n}\right), & \text { if }(m \neq k, l) \text { and }(n=l) ; \\
-\frac{i}{2}\left(q_{m} q_{n} p_{m} p_{n}+p_{m} p_{n} q_{m} q_{n}\right), & \text { otherwise. }\end{cases}
\end{aligned}
$$

Insertion of these commutators into Eq. (4.66) leads to:

$$
\begin{aligned}
& \frac{1}{2} \sum_{(m, n),(k, l)}^{\prime}\left\langle n_{F}\left|\left[S_{8 ; F m n}, \mathcal{H}_{3 ; F k l}\right]\right| n_{F}\right\rangle= \\
& \quad-\frac{i}{2} \sum_{(m, n),(k, l)}^{\prime} \kappa_{F m n} \kappa_{F k l} \theta_{F m n} q_{m} q_{n} q_{k} q_{l}+i \sum_{(m, n, k, l)}^{\prime} \kappa_{F m n} \kappa_{F k l} \frac{\omega_{F} \omega_{m} \omega_{n}}{\Omega_{F m n}} p_{m} p_{n} q_{k} q_{l} \\
& \quad-i \sum_{(m, n),(m, k)}^{\prime} \kappa_{F m n} \kappa_{F k m} \theta_{m n F}\left(n_{F}+\frac{1}{2}\right) q_{n} q_{k}-i \sum_{(m, n),(n, k)}^{\prime} \kappa_{F m n} \kappa_{F k n} \theta_{n m F}\left(n_{F}+\frac{1}{2}\right) q_{m} q_{k} \\
& \quad+i \sum_{m,(n, k, l)}^{\prime} \kappa_{F m n} \kappa_{F k l} \frac{\omega_{F} \omega_{m} \omega_{n}}{\Omega_{F m n}}\left\{p_{n} q_{l} \delta_{m, k}+p_{n} q_{k} \delta_{m, l}\right\}\left(q_{m} p_{m}+p_{m} q_{m}\right) \\
& \quad+i \sum_{(m, n)}^{\prime} \kappa_{F m n} \kappa_{F m n} \frac{\omega_{F} \omega_{m} \omega_{n}}{\Omega_{F m n}}\left(q_{m} q_{n} p_{m} p_{n}+p_{m} p_{n} q_{m} q_{n}\right),
\end{aligned}
$$

and this reduces to:

$$
\begin{aligned}
& \frac{1}{2} \sum_{(m, n),(k, l)}^{\prime}\left\langle n_{F}\left|\left[S_{8 ; F m n}, \mathcal{H}_{3 ; F k l}\right]\right| n_{F}\right\rangle= \\
& \quad-\frac{i}{2} \sum_{(m, n),(k, l)}^{\prime} \kappa_{F m n} \kappa_{F k l}\left\{\theta_{F m n} q_{m} q_{n} q_{k} q_{l}-\frac{\omega_{F} \omega_{m} \omega_{n}}{\Omega_{F m n}}\left(p_{m} p_{n} q_{k} q_{l}+q_{k} q_{l} p_{m} p_{n}\right)\right\} \\
& \quad-2 i \sum_{(m, n),(m, k)}^{\prime} \kappa_{F m n} \kappa_{F k m} \theta_{m n F}\left(n_{F}+\frac{1}{2}\right) q_{n} q_{k},
\end{aligned}
$$

which is the last term needed to evaluate Eq. (4.60).

Insertion of Eqs. (4.61), (4.65), and (4.70) in Eq. (4.60) results in:

$$
\begin{aligned}
& \sum_{(k, l)}^{\prime}\left\langle n_{F}\left|\left[S^{(1)}, \mathcal{H}_{8 ; F k l}\right]\right| n_{F}\right\rangle= \\
& \quad \frac{i}{2} \sum_{(k, l)}^{\prime} \frac{\kappa_{F F F} \kappa_{F k l}}{\omega_{F}}\left(n_{F}+\frac{1}{2}\right) q_{k} q_{l}+2 i \sum_{(k, l)}^{\prime} \frac{\kappa_{F k k} \kappa_{F k l} \omega_{k}}{\left(4 \omega_{k}^{2}-\omega_{F}^{2}\right)}\left(n_{F}+\frac{1}{2}\right) q_{k} q_{l} \\
& \quad+\frac{i}{2} \sum_{m,(k, l)}^{\prime} \frac{\kappa_{F m m} \kappa_{F k l}}{\omega_{F}\left(4 \omega_{m}^{2}-\omega_{F}^{2}\right)}\left\{\left(2 \omega_{m}^{2}-\omega_{F}^{2}\right) q_{k} q_{l} q_{m}^{2}+\omega_{m}^{2}\left(q_{l} q_{k} p_{k}^{2}+p_{k}^{2} q_{l} q_{k}\right)\right\}
\end{aligned}
$$




$$
\begin{aligned}
& -\frac{i}{2} \sum_{(m, n),(k, l)}^{\prime} \kappa_{F m n} \kappa_{F k l}\left\{\theta_{F m n} q_{m} q_{n} q_{k} q_{l}-\frac{\omega_{F} \omega_{m} \omega_{n}}{\Omega_{F m n}}\left(p_{m} p_{n} q_{k} q_{l}+q_{k} q_{l} p_{m} p_{n}\right)\right\} \\
& -2 i \sum_{(m, k),(m, l)}^{\prime} \kappa_{F m k} \kappa_{F l m} \theta_{m k F}\left(n_{F}+\frac{1}{2}\right) q_{k} q_{l} .
\end{aligned}
$$

Collecting terms and using the definition of $\theta_{k l m}$ provides,

$$
\begin{aligned}
& \sum_{(k, l)}^{\prime}\left\langle n_{F}\left|\left[S^{(1)}, \mathcal{H}_{8 ; F k l}\right]\right| n_{F}\right\rangle= \\
& \quad \frac{i}{2} \sum_{(k, l)}^{\prime}\left\{\frac{\kappa_{F F F} \kappa_{F k l}}{\omega_{F}}-4 \kappa_{F k k} \kappa_{F k l} \theta_{k k F}\right\}\left(n_{F}+\frac{1}{2}\right) q_{k} q_{l} \\
& \quad-2 i \sum_{(m, k),(m, l)}^{\prime} \kappa_{F m k} \kappa_{F m l} \theta_{m k F}\left(n_{F}+\frac{1}{2}\right) q_{k} q_{l} \\
& \quad-\frac{i}{2} \sum_{m,(k, l)}^{\prime} \kappa_{F m m} \kappa_{F k l}\left\{\theta_{F m m} q_{m}^{2} q_{k} q_{l}-\frac{\omega_{F} \omega_{m}^{2}}{\Omega_{F m m}}\left(q_{l} q_{k} p_{k}^{2}+p_{k}^{2} q_{l} q_{k}\right)\right\} \\
& \quad-\frac{i}{2} \sum_{(m, n),(k, l)}^{\prime} \kappa_{F m n} \kappa_{F k l}\left\{\theta_{F m n} q_{m} q_{n} q_{k} q_{l}-\frac{\omega_{F} \omega_{m} \omega_{n}}{\Omega_{F m n}}\left(p_{m} p_{n} q_{k} q_{l}+q_{k} q_{l} p_{m} p_{n}\right)\right\}
\end{aligned}
$$

This can be further simplified,

$$
\begin{aligned}
& \sum_{(k, l)}^{\prime}\left\langle n_{F}\left|\left[S^{(1)}, \mathcal{H}_{8 ; F k l}\right]\right| n_{F}\right\rangle= \\
& \quad \frac{i}{2} \sum_{(k, l)}^{\prime}\left\{\frac{\kappa_{F F F} \kappa_{F k l}}{\omega_{F}}-4 \kappa_{F k k} \kappa_{F k l} \theta_{k k F}\right\}\left(n_{F}+\frac{1}{2}\right) q_{k} q_{l} \\
& \quad-2 i \sum_{(m, k),(m, l)}^{\prime} \kappa_{F m k} \kappa_{F m l} \theta_{m k F}\left(n_{F}+\frac{1}{2}\right) q_{k} q_{l} \\
& \quad-\frac{i}{2} \sum_{m, n,(k, l)}^{\prime} \kappa_{F m n} \kappa_{F k l}\left\{\theta_{F m n} q_{m} q_{n} q_{k} q_{l}-\frac{\omega_{F} \omega_{m} \omega_{n}}{\Omega_{F m n}}\left(p_{m} p_{n} q_{k} q_{l}+q_{k} q_{l} p_{m} p_{n}\right)\right\},
\end{aligned}
$$

providing the desired result for this subsection.

\subsubsection{Evaluation of $\left\langle n_{F}\left|\mathcal{H}_{0}^{(2)}\right| n_{F}\right\rangle$}

In the last section all of the commutator terms in Eq. (4.31) were evaluated onto the $\left|n_{F}\right\rangle$ state. In this section we evaluate the remaining term needed in that expression, namely,

$$
\left\langle n_{F}\left|\mathcal{H}_{0}^{(2)}\right| n_{F}\right\rangle=\left\langle n_{F}\left|\left(\frac{1}{24} \kappa_{F F F} q_{F}^{4}+\frac{1}{4} \sum_{k, l}^{\prime} \kappa_{F F k l} q_{F}^{2} q_{k} q_{l}+\frac{1}{24} \sum_{k, l, m, n}^{\prime} \kappa_{k l m n} q_{k} q_{l} q_{m} q_{n}\right)\right| n_{F}\right\rangle
$$


where terms odd in $F$ have already been dropped since they vanish inside the matrix element. The first term in this expression is the same as in standard CVPT while the remaining terms, may be obtained trivially, resulting in

$$
\begin{aligned}
\left\langle n_{F}\left|\mathcal{H}_{0}^{(2)}\right| n_{F}\right\rangle= & \frac{1}{16} \kappa_{F F F F}\left\{\left(n_{F}+\frac{1}{2}\right)^{2}+\frac{1}{4}\right\}+\frac{1}{4} \sum_{k, l}^{\prime} \kappa_{F F k l}\left(n_{F}+\frac{1}{2}\right) q_{k} q_{l} \\
& +\frac{1}{24} \sum_{k, l, m, n}^{\prime} \kappa_{k l m n} q_{k} q_{l} q_{m} q_{n} .
\end{aligned}
$$

\subsubsection{The Second Order Hamiltonian Operator}

To second order in the perturbation, the Hamiltonian is

$$
\begin{aligned}
\mathcal{H}^{(2)} \equiv & \left\langle n_{F}\right| \mathcal{H}_{0}^{(0)}+\mathcal{H}_{\mathrm{I}}^{(1)}+\mathcal{H}_{0}^{(2)}+\frac{i}{2}\left[S^{(1)},\left\{2 \mathcal{H}_{1}^{(1)}+\frac{1}{6} \mathcal{H}_{3 ; F F F}\right.\right. \\
& \left.\left.+\frac{1}{2} \sum_{k}^{\prime}\left(\mathcal{H}_{5 ; F F k}+\mathcal{H}_{5 ; F k k}\right)+\frac{1}{2} \sum_{(k, l)}^{\prime} \mathcal{K}_{i ; F k l}^{\prime}\right\}\right]\left|n_{F}\right\rangle
\end{aligned}
$$

Substitution using Eqs. (4.2), (4.27), (4.33), (4.40), (4.59), (4.73), and (4.75) results in:

$$
\begin{aligned}
\mathcal{H}^{(2)}= & \omega_{F}\left(n_{F}+\frac{1}{2}\right)+\frac{1}{2} \sum_{k}^{\prime} \omega_{k}\left(p_{k}^{2}+q_{k}^{2}\right)+\frac{1}{6} \sum_{k, l, m}^{\prime} \kappa_{k l m} q_{k} q_{l} q_{m}+\frac{1}{24} \sum_{k, l, m, n}^{\prime} \kappa_{k l m n} q_{k} q_{l} q_{m} q_{n} \\
& +\frac{1}{16} \kappa_{F F F F}\left\{\left(n_{F}+\frac{1}{2}\right)^{2}+\frac{1}{4}\right\}+\frac{1}{4} \sum_{k, l}^{\prime} \kappa_{F F k l}\left(n_{F}+\frac{1}{2}\right) q_{k} q_{l} \\
& -\frac{1}{2} \sum_{k}^{\prime} \frac{\kappa_{F F k}}{\omega_{k}}\left(n_{F}+\frac{1}{2}\right) A_{k}^{(2)}-\frac{\kappa_{F F F}^{2}}{36 \omega_{F}}\left[\frac{15}{4}\left(n_{F}+\frac{1}{2}\right)^{2}+\frac{7}{16}\right] \\
& +\frac{1}{8} \sum_{k, l}^{\prime} \kappa_{F k l} \kappa_{F F F}\left(\theta_{F k l} q_{k} q_{l}-\frac{2 \omega_{F} \omega_{k} \omega_{l}}{\Omega_{F k l}} p_{k} p_{l}\right)\left(n_{F}+\frac{1}{2}\right) \\
& -\frac{3}{64} \sum_{k}^{\prime} \frac{\kappa_{F F k} \kappa_{F F k}}{\omega_{k}\left(4 \omega_{F}^{2}-\omega_{k}^{2}\right)}\left\{\frac{4}{3}\left(8 \omega_{F}^{2}-3 \omega_{k}^{2}\right)\left(n_{F}+\frac{1}{2}\right)^{2}-\omega_{k}^{2}\right\} \\
& -\frac{1}{8} \sum_{k}^{\prime} \frac{\kappa_{F F F} \kappa_{F k k}}{\omega_{F}}\left(n_{F}+\frac{1}{2}\right) q_{k}^{2} \\
& +\frac{1}{2} \sum_{k, l}^{\prime}\left\{\kappa_{F F k} \kappa_{F F l} \theta_{F F l}+\kappa_{F k l} \kappa_{F k k} \theta_{k l F}\right\}\left(n_{F}+\frac{1}{2}\right) q_{k} q_{l} \\
& +\frac{1}{8} \sum_{k, l, m}^{\prime} \kappa_{F l m} \kappa_{F k k}\left\{\theta_{F l m} q_{k}^{2} q_{l} q_{m}-\omega_{F} \omega_{l} \omega_{m} \Omega_{F l m}^{-1}\left(p_{l} p_{m} q_{k}^{2}+q_{k}^{2} p_{l} p_{m}\right)\right\} \\
& -\frac{1}{8} \sum_{(k, l)}^{\prime}\left\{\frac{\kappa_{F F F} \kappa_{F k l}}{\omega_{F}}-4 \kappa_{F k k} \kappa_{F k l} \theta_{k k F}\right\}\left(n_{F}+\frac{1}{2}\right) q_{k} q_{l} \\
& +\frac{1}{2} \sum_{(m, k),(m, l)}^{\prime} \kappa_{F m k} \kappa_{F m l} \theta_{m k F}\left(n_{F}+\frac{1}{2}\right) q_{k} q_{l}
\end{aligned}
$$




$$
+\frac{1}{8} \sum_{m, n,(k, l)}^{\prime} \kappa_{F m n} \kappa_{F k l}\left\{\theta_{F m n} q_{m} q_{n} q_{k} q_{l}-\frac{\omega_{F} \omega_{m} \omega_{n}}{\Omega_{F m n}}\left(p_{m} p_{n} q_{k} q_{l}+q_{k} q_{l} p_{m} p_{n}\right)\right\}
$$

Rearranging and combining terms leads to:

$$
\begin{aligned}
\mathcal{H}^{(2)}= & \frac{1}{2} \sum_{k}^{\prime} \omega_{k}\left(p_{k}^{2}+q_{k}^{2}\right)+\frac{1}{6} \sum_{k, l, m}^{\prime} \kappa_{k l m} q_{k} q_{l} q_{m}+\frac{1}{24} \sum_{k, l, m, n}^{\prime} \kappa_{k l m n} q_{k} q_{l} q_{m} q_{n} \\
& +\left\{\frac{1}{64} \kappa_{F F F}-\frac{7 \kappa_{F F F}^{2}}{576 \omega_{F}}+\frac{3}{64} \sum_{k}^{\prime} \frac{\kappa_{F F k} \kappa_{F F k} \omega_{k}}{\left(4 \omega_{F}^{2}-\omega_{k}^{2}\right)}\right\}+\omega_{F}\left(n_{F}+\frac{1}{2}\right) \\
& +\left\{\frac{1}{16} \kappa_{F F F}-\frac{5 \kappa_{F F F}^{2}}{48 \omega_{F}}-\frac{1}{16} \sum_{k}^{\prime} \kappa_{F F k}^{2} \frac{\left(8 \omega_{F}^{2}-3 \omega_{k}^{2}\right)}{\omega_{k}\left(4 \omega_{F}^{2}-\omega_{k}^{2}\right)}\right\}\left(n_{F}+\frac{1}{2}\right)^{2} \\
& +\frac{1}{4} \sum_{k, l}^{\prime} \kappa_{F F k l}\left(n_{F}+\frac{1}{2}\right) q_{k} q_{l}-\frac{1}{2} \sum_{k}^{\prime} \frac{\kappa_{F F k}}{\omega_{k}}\left(n_{F}+\frac{1}{2}\right) A_{k}^{(2)} \\
& +\frac{1}{8} \sum_{k, l}^{\prime} \kappa_{F k l} \kappa_{F F F}\left\{\left(\theta_{F k l}-\omega_{F}^{-1}\right) q_{k} q_{l}-\frac{2 \omega_{F} \omega_{k} \omega_{l}}{\Omega_{F k l}} p_{k} p_{l}\right\}\left(n_{F}+\frac{1}{2}\right) \\
& +\frac{1}{2} \sum_{k, l}^{\prime} \kappa_{F F k} \kappa_{F F l} \theta_{F F l}\left(n_{F}+\frac{1}{2}\right) q_{k} q_{l}+\frac{1}{2} \sum_{m, k, l}^{\prime} \kappa_{F m k} \kappa_{F m l} \theta_{m k F}\left(n_{F}+\frac{1}{2}\right) q_{k} q_{l} \\
& +\frac{1}{8} \sum_{m, n, k, l}^{\prime} \kappa_{F m n} \kappa_{F k l}\left\{\theta_{F m n} q_{m} q_{n} q_{k} q_{l}-\omega_{F} \omega_{m} \omega_{n} \Omega_{F m n}^{-1}\left(p_{m} p_{n} q_{k} q_{l}+q_{k} q_{l} p_{m} p_{n}\right)\right\} .
\end{aligned}
$$

This reduces further to the final expression:

$$
\begin{aligned}
\mathcal{H}^{(2)}= & \frac{1}{2} \sum_{k}^{\prime} \omega_{k}\left(p_{k}^{2}+q_{k}^{2}\right)+\frac{1}{6} \sum_{k, l, m}^{\prime} \kappa_{k l m} q_{k} q_{l} q_{m}+\frac{1}{24} \sum_{k, l, m, n}^{\prime} \kappa_{k l m n} q_{k} q_{l} q_{m} q_{n} \\
& +\left\{\frac{1}{64} \kappa_{F F F F}-\frac{7 \kappa_{F F F}^{2}}{576 \omega_{F}}+\frac{3}{64} \sum_{k}^{\prime} \frac{\kappa_{F F k} \kappa_{F F k} \omega_{k}}{\left(4 \omega_{F}^{2}-\omega_{k}^{2}\right)}\right\}+\omega_{F}\left(n_{F}+\frac{1}{2}\right) \\
& +\left\{\frac{1}{16} \kappa_{F F F F}-\frac{5 \kappa_{F F F}^{2}}{48 \omega_{F}}-\frac{1}{16} \sum_{k}^{\prime} \kappa_{F F k}^{2} \frac{\left(8 \omega_{F}^{2}-3 \omega_{k}^{2}\right)}{\omega_{k}\left(4 \omega_{F}^{2}-\omega_{k}^{2}\right)}\right\}\left(n_{F}+\frac{1}{2}\right)^{2} \\
& -\frac{1}{2}\left(n_{F}+\frac{1}{2}\right)\left[\frac{1}{2} \sum_{k, l}^{\prime} \kappa_{F k l} \kappa_{F F F} \frac{\omega_{F} \omega_{k} \omega_{l}}{\Omega_{F k l}} p_{k} p_{l}-\sum_{k, l}^{\prime}\left\{\frac{1}{2} \kappa_{F F k l}+\kappa_{F F k} \kappa_{F F l} \theta_{F F l}\right.\right. \\
& \left.\left.+\frac{1}{4} \kappa_{F k l} \kappa_{F F F}\left(\theta_{F k l}-\omega_{F}^{-1}\right)+\sum_{m}^{\prime}\left(\kappa_{F m k} \kappa_{F m l} \theta_{m k F}-\frac{1}{2} \kappa_{F F m} \kappa_{k l m} \omega_{m}^{-1}\right)\right\} q_{k} q_{l}\right] \\
& +\frac{1}{8} \sum_{m, n, k, l}^{\prime} \kappa_{F m n} \kappa_{F k l}\left\{\theta_{F m n} q_{m} q_{n} q_{k} q_{l}-\omega_{F} \omega_{m} \omega_{n} \Omega_{F m n}^{-1}\left(p_{m} p_{n} q_{k} q_{l}+q_{k} q_{l} p_{m} p_{n}\right)\right\} .
\end{aligned}
$$

For example, in the 2-dimensional case, we obtain:

$$
\mathcal{H}^{(2)}=\frac{1}{2} \omega_{1}\left(p_{1}^{2}+q_{1}^{2}\right)+\frac{1}{6} \kappa_{111} q_{1}^{3}+\frac{1}{24} \kappa_{1111} q_{1}^{4}
$$




$$
\begin{aligned}
& +\frac{1}{8} \kappa_{F 11}^{2}\left\{\theta_{F 11} q_{1}^{4}-\omega_{F} \omega_{1}^{2} \Omega_{F 11}^{-1}\left(p_{1}^{2} q_{1}^{2}+q_{1}^{2} p_{1}^{2}\right)\right\} \\
& +\left\{\frac{1}{64} \kappa_{F F F}-\frac{7}{576} \frac{\kappa_{F F F}^{2}}{\omega_{F}}+\frac{3}{64} \frac{\kappa_{F F 1}^{2} \omega_{1}}{\left(4 \omega_{F}^{2}-\omega_{1}^{2}\right)}\right\}+\omega_{F}\left(n_{F}+\frac{1}{2}\right) \\
& +\left(\frac{1}{16} \kappa_{F F F}-\frac{5 \kappa_{F F F}^{2}}{48 \omega_{F}}-\frac{1}{16} \kappa_{F F 1}^{2} \frac{\left(8 \omega_{F}^{2}-3 \omega_{1}^{2}\right)}{\omega_{1}\left(4 \omega_{F}^{2}-\omega_{1}^{2}\right)}\right)\left(n_{F}+\frac{1}{2}\right)^{2} \\
& +\frac{1}{2}\left(n_{F}+\frac{1}{2}\right)\left[\frac{1}{2} \kappa_{F 11} \kappa_{F F F} \omega_{F} \omega_{1}^{2} \Omega_{F 11}^{-1}\left(p_{1}^{2}-q_{1}^{2}\right)+\left\{\frac{1}{2} \kappa_{F F 11}\right.\right. \\
& \left.\left.\quad-\frac{1}{2} \kappa_{F 11} \kappa_{F F F} \omega_{F}^{-1}-\frac{1}{2} \kappa_{F F 1} \kappa_{111} \omega_{1}^{-1}+\kappa_{F F 1}^{2} \theta_{F F 1}+\kappa_{F 11}^{2} \theta_{11 F}\right\} q_{1}^{2}\right] .
\end{aligned}
$$

where 1 labels the perpendicular mode.

The multidimensional mixed-diagonalization Hamiltonian (4.79) and its 2dimensional trivialization, Eq. (4.80), are in the form that has been sought. The terms in the original Hamiltonian, which involve only the $F-1$ degrees of freedom not treated perturbatively and the standard perturbation theory result for a 1-dimensional vibrational system as applied to mode $F$, are all contained therein. If there were no coupling between mode $F$ and the rest, there would be no additional terms, and this is manifested in the mixed-diagonalization Hamiltonian. In general there is anharmonic coupling between these modes, and the additional terms in the mixed-diagonalization Hamiltonian represent operators in the reduced $F-1$ dimensional space whose coefficients are parametrized by the quantum number of mode F.

\subsubsection{Summary}

In this section, perturbation theory has been used to obtain a Hamiltonian in which at least one normal mode corresponds to a good quantum number of the system which is only partly coupled to the remaiming degrees of freedom. Although on'y the results for the case with one such mode is explicitly, the general result follows readily by repeated application. (The only detail that need be recognized in carrying out this recursive procedure is that the terms in the mixed-diagonalization Hamiltonian which have arisen during a given recursion step should be treated as second order terms in subsequent recursion steps.) In particular, it is an easy - but lengthy! calculation to check that in the limit that this recursion is applied $F$ times to an 
$F$-dimensional Hamiltonian, the standard CVPT expressions ${ }^{107}$ obtain.

Use of the correspondence relation - e.g. Eq. (2.15) - connecting $n_{F}$ to the generalized barrier penetration integral $\theta$ results in an effective Hamiltonian, $\mathcal{H}(\theta)$, which can be used in the semiclassical formulae of Sec. 3.2 to obtain thermal rate constants. For example, in order to compute the rate,

$$
k(T)=\left(2 \pi \hbar Q_{r} \beta\right)^{-1} \int_{-\infty}^{\infty} d \theta \frac{1}{2} \operatorname{sech}^{2}(\theta) \sum_{n^{\natural}} \mathrm{e}^{-\beta E\left(n^{4}, \theta\right)},
$$

one needs only diagonalize the effective Hamiltonian for each value of $\theta$ in a quadrature scheme. By analogy with the standard rate formula involving the Boltzmann average of the CRP, this result suggests that the CRP can also be more accurately computed by first diagonalizing the mixed-diagonalization Hamiltonian (as one does when there are Fermi resonances), and then performing a numerical inversion to obtain $\theta_{j}(E)$ where $j$ is now a collective index for the eigenvalues of $\mathcal{H}(\theta)$. This extends the view of the semiclassical calculation discussed in Seideman and Miller ${ }^{112}$ in which the eigenergies of the SCTST are shown to be related to Siegert ${ }^{144-146}$ eigenvalues.

\subsection{Application to Collinear $\mathrm{H}+\mathrm{H}_{2} \rightarrow \mathrm{H}_{2}+\mathrm{H}$}

In order to apply the mixed-diagonalization Hamiltonian obtained in the previous section all that is needed are the force constants of the potential at the transition state. Since this is precisely the information which is needed to obtain the "spectroscopic constants" for the full perturbation theory approach of the SCTST employed in earlier chapters, this information is readily available for each of the systems discussed thus far, in which tisere are no degenerate vibrational modes. However, for a one dimensional system there are no perpendicular modes, and it is therefore necessary to apply the theory to a system with dimensionality greater than or equal to two. The smallest system studied thus far which meets this criterion is the colinear $\mathrm{H}+\mathrm{H}_{2} \rightarrow \mathrm{H}_{2}+\mathrm{H}$ reaction for which the force constants may be found in Ref. 104. This system will thus serve to illustrate the use of the mixed-diagonalization Hamiltonian in obtaining the CRP, in principle, a stricter test than that afforded by a thermal rate calculation, as it involves less averaging. 


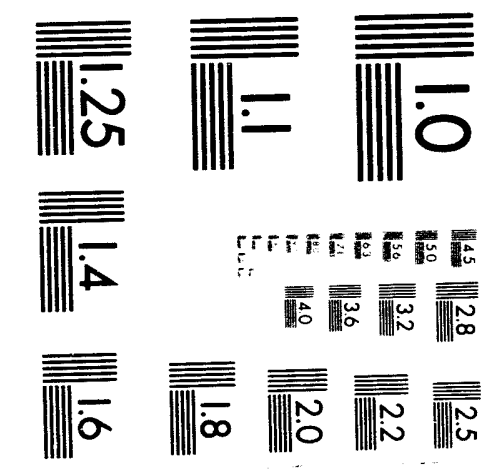



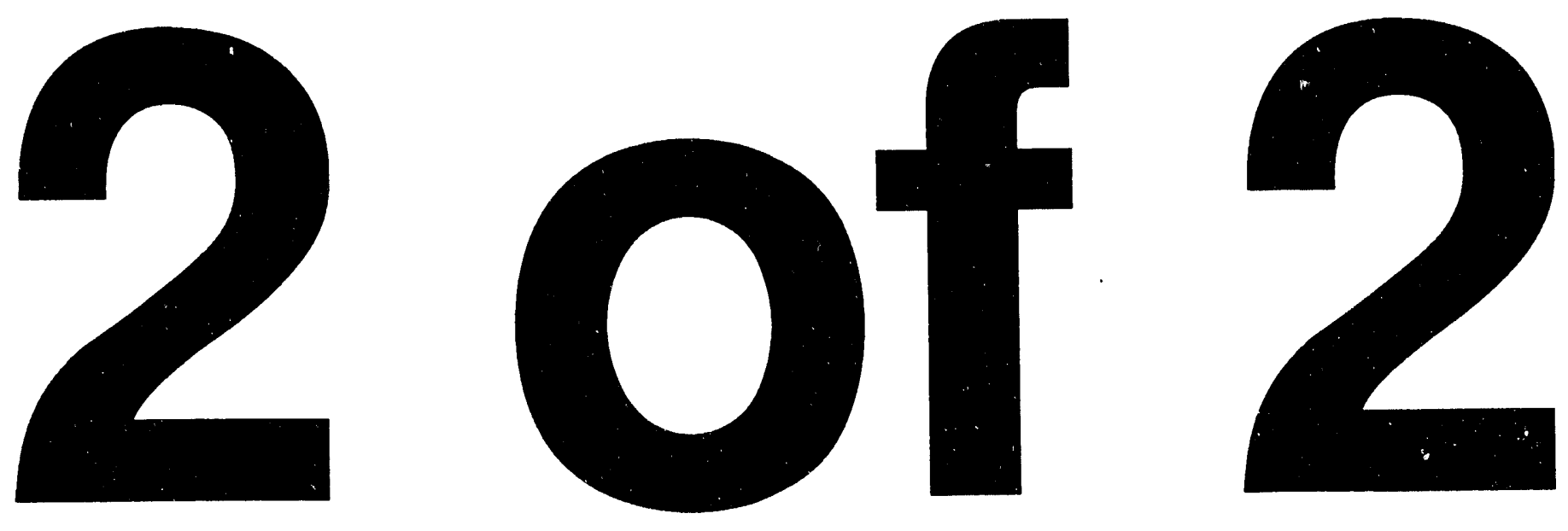

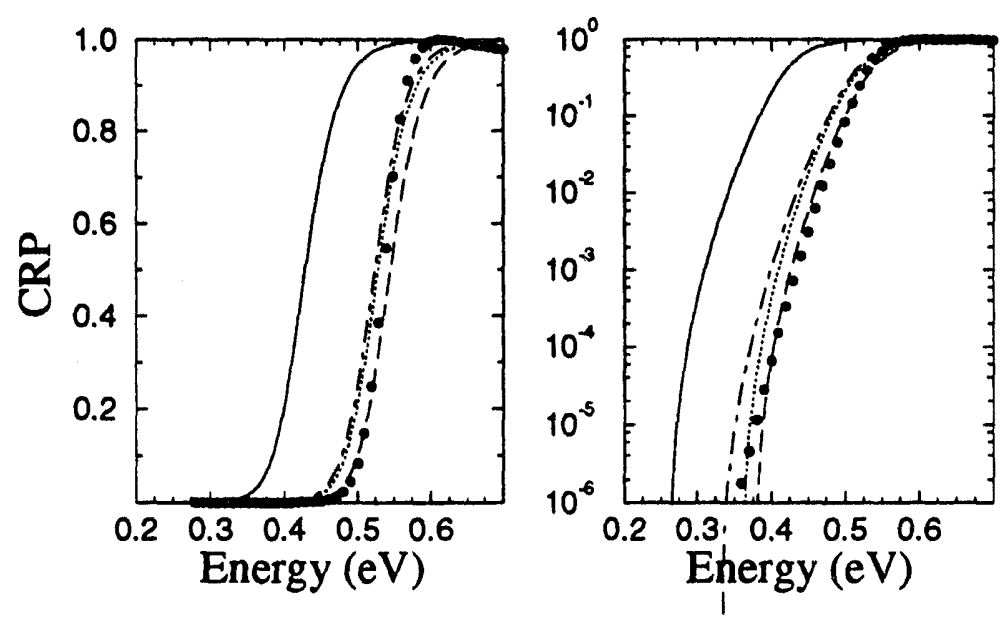

Figure 4.1: The CRP for the collinear $\mathrm{H}+\mathrm{H}_{2}$ reaction is presented for several cases: exact results ${ }^{2}$ (points); anharmonic SCTST with $\bar{E}_{0}=0$ (short-dashed curve); anharmonic SCTST with $\bar{E}_{0}=-138.3 \mathrm{~cm}^{-1}$ (dotted curve); anharmonic SCTST with $\bar{E}_{0}=-936.6 \mathrm{~cm}^{-1}$ (solid curve); and SCTST with mixed-diagonalization Hamiltonian (dot-dashed curve).

In Fig. 4.1, the mixed-diagonalization result for the CRP in the low energy regime is compared to the exact results of Bondi et al ${ }^{2}$ and to the anharmonic SCTST. (Note that the exact results were actually obtained for the LSTH surface; but these differ from the DMBE results by less than the resolution of the figure.) In the figure, several anharmonic SCTST CRPs are presented and these differ only by the choice of $\bar{E}_{0}:(i)$ if the perturbation theory calculation is performed only for the 2-dimensional collinear system, the resulting $\bar{E}_{0}=-936.6 \mathrm{~cm}^{-1} ;$ (ii) if instead, $E_{0}$ is taken from the full-dimensional calculations, then $E_{0}=-138.3 \mathrm{~cm}^{-1}$ for the case in which the Fermi resonant term is excluded; and (iii) if $\bar{E}_{0}$ is ignored and set to 0, i.e., the result presented in Fig. 2.2. For the mixed-diagonalization calculation, the result follows directly from the force constants and there is no ad hoc modification of the Hamiltonian.

The results in Fig. 4.1 suggest that the anharmonic SCTST provides an approximately correct non-exponential behavior, but the correction to the threshold energy as provided by $\vec{E}_{0}$ is incorrect. This latter error is evidently due to the perturbation theory, and the mixed-diagonalization offers an improvement of the threshold 
error although it is not in perfect agreement with the exact result. It should be emphasized, though, that the collinear $\mathrm{H}+\mathrm{H}_{2}$ reaction is highly anharmonic - as may be seen from the large anharmonic "spectroscopic constants" in Table 2.1 - and it is also one in which quantum mechanical effects predominate as is exhibited by the resonance features in the CRP. Thus the fact that the SCTST results using mixeddiagonalization are in reasonable agreement with the exact results is encouraging, and further work is in progress.

\subsection{Concluding Remarks}

The mixed-diagonalization method developed in this chapter appears to be quite general. It could be used, for example, in order to construct effective Hamiltonians for bound molecules with unly a few wide amplitude motions. The only difficulty in this procedure is the identification of the limited subspace of quasi-bound modes which are to be treated only perturbatively.

In this chapter, the mixed-diagonalization approach has been explicitly used to construct the semiclassical Hamiltonian $\mathcal{H}(\theta)$ needed in the SCTST thermal rate expression, and in the computation of the SCTST CRP. This application may seem counter-intuitive as the scattering motion along the reaction path should, in principle, involve the widest amplitude motion, and thus by the arguments of the bound-state paradigm should be the one in which perturbation theory should be the least effective. From a practical point of view, however, $\theta$ corresponds to the only quantum number that need be "good" and so the construction requires a local treatment of this degree of freedom while the non-perturbative treatment of the remaining degrees of freedom allows for a more accurate treatment of their motion.

Moreover, returning to classical mechanics, if a Hamiltonian with $F$ degrees of freedom is not integrable but contains a constant of the motion which can be associated with the reactive degree of freedom, then a canonical transformation will produce a new Hamiltonian parametrized by a good action variable which is bound in the remaining degrees of freedom. This good action is the one which is associated with $\theta$ in the situation where there exist $F-1$ additional constants of the motion leading to 
an integrable Hamiltonian in Miller's good-action angle variable theory. Thus by an analogous extension of Miller's theory when the good action-angle variables are only locally known, the locally "good" action variable for the reactive degree of freedom of the otherwise nonintegrable Hamiltonian can be associated with $\theta$. The error incurred with respect to the unbound motion should be of the same order as that when all $F$ modes are treated locally; here, however, the bound motions can be treated exactly. Thus we obtain a generalization of Miller's theory which, in principle, should provide systematically better results as the number of perpendicular modes which are treated exactly is increased.

The preliminary results seen in the application to the collinear $\mathrm{H}+\mathrm{H}_{2}$ reaction are encouraging, but more work still needs to be done. For example, if the potential energy surface is known only to second order, there may be no advantage to performing a more "accurate" calculation than the anharmonic SCTST presented in previous chapters, as is evident from the discussion of the Eckart barrier CRP in Sec. 2.4.1. 


\section{Chapter 5}

\section{A Random Matrix/Transition State Theory for the Probability Distributions of Unimolecular Decay Rates}

\subsection{Introduction}

In a remarkable series of experiments, Polik et al..$^{96,21}$ determined the unimolecular reaction rates for the decomposition of formaldehyde in its ground electronic state,

$$
\mathrm{D}_{2} \mathrm{CO} \longrightarrow \mathrm{D}_{2}+\mathrm{CO}
$$

for individual quantum (i.e., rovibrational) states of the reactant molecule. Although standard statistical theory (i.e., RRKM, microcanonical transition state theory, etc. ${ }^{12,147,45}$ ) provides a good description of the rate as a function of the energy of the molecule on the average, the decay rates of individual quantum states with energies in a given energy interval show significant fluctuations about the average rate for that energy (interval). Fig. 5.1 shows a schematic depiction of this situation. 


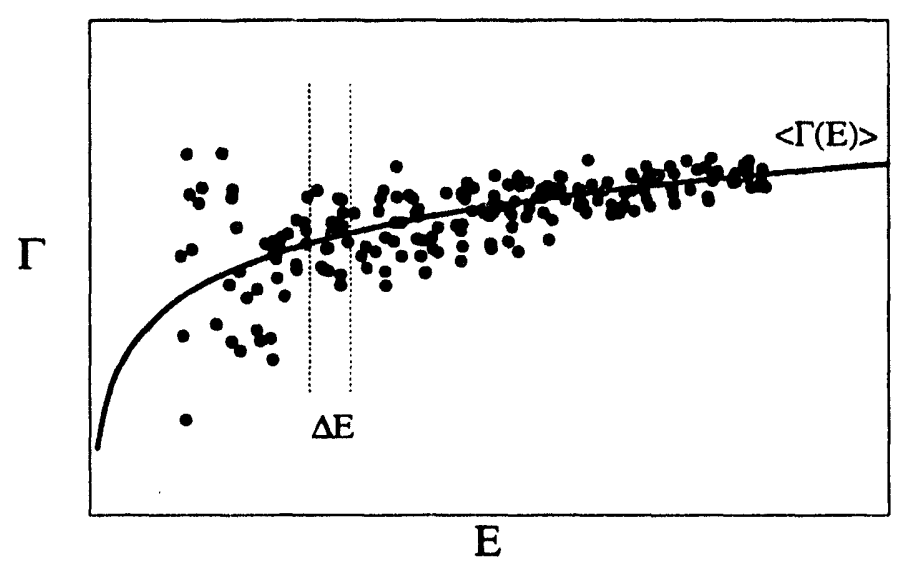

Figure 5.1: Unimolecular decay rate vs. energy. The points indicate the decay rates and energies for individual quantum states of the reactant molecule. $\langle\Gamma(E)\rangle$ is the average rate averaged over all the states in a given narrow energy interval, $\delta E$.

As described in Sec. 1.2.3, the standard model which has been used to describe the fluctuations has been the Porter-Thomas model ${ }^{63}$ or its generalization which results in the $\chi^{2}$ distributions. However, the experimental results of Polik et al. did not agree with the best fit $\chi^{2}$ distributions, and this led them to posit a generalization of the Porter-Thomas model..$^{98}$ A more rigorous derivation of this model was later obtained and will be described in Sec. 5.2. ${ }^{100}$ The essential assumptions of this statistical model are that the states in the quasi-bound region of the potential energy surface be non-overlapping and strongly mixed. The first assumption is clearly satisfied if the experiments resolve individual quantum states. By strongly mixed we mean that the system behaves like the Gaussian Orthogonal Ensemble (GOE) of random matrix theory. ${ }^{69,61}$ Physically, this latter requirement is equivalent to the assumption that the expansion coefficients (in some generic basis) of the eigenstates in a given energy interval all behave as independent random variables. The good agreement of various spectral measures between the experimental results and predictions of the Gaussian Orthogonal Ensemble indicates that the eigenstates of $\mathrm{D}_{2} \mathrm{CO}$ are indeed well described as strongly mixed. ${ }^{99}$

It is interesting to note that the Porter-Thomas model has previously been obtained within the formalism of random matrix theory, ${ }^{61}$ but stopped short of look- 
ing at the decay rate matrix itself, which is the object that has afforded us progress. In particular, the interpretation of the $\chi^{2}$ parameter, $\nu$, as an effective number of channels is given a rigorous footing within this formalism, and the noninteger values it can attain are a manifestation of quantum mechanical tunneling. Both this fact and the wealth of distributions that this statistical model can represent are illustrated in Sec. 5.3. In Sec. 5.4, this model is converted into a predictive theory, with the demonstration that the eigenvalues of the decay rate matrix are related to the semiclassical transition state transmission probabilities described in earlier chapters. This theory is consequently called a random matrix / transition state (RM/TST) theory.

In practice, it may often be the case that there will be dynamical symmetries - e.g. angular momentum conservation - which are obeyed throughout the dynamics; but this is incompatible with the strong mixing assumption in RM/TST theory. Nonetheless, the strong mixing assumption can be used to describe each of the symmetry manifolds as is presented in Sec. 5.5. Thus the only adjustable parameter within the RM/TST theory is the set of symmetries that are assumed to be obeyed throughout the reaction dynamics.

The application of the RM/TST methodology to the formaldehyde dissociation (5.1) and subsequent interpretation of the experimental result is presented in the next chapter.

\subsection{The RM/TST Model}

\subsubsection{The Construction of the Model}

A system undergoing unimolecular dissociation can, in general, be described by an effective Hamiltonian matrix ${ }^{148,149}$ of the form

$$
\mathcal{H}_{m, m^{\prime}}^{\mathrm{eff}}=\mathcal{H}_{m, m^{\prime}}-\frac{i}{2} \Gamma_{m, m^{\prime}}
$$

where $\{|m\rangle\}$ is some bound-state $\left(\mathbf{L}^{2}\right)$ basis which spans the (Hilbert) space of the reactant molecule. The real symmetric matrix $\mathcal{H}_{m, m^{\prime}}$ describes coupling (anharmonic, coriolis, etc.) in the reactant molecule. The decay rate matrix $\Gamma_{m, m^{\prime}}$ describes the 
coupling of the bound states $\{|n\rangle\}$ to a continuum of unspecified scattering states of the dissociated molecule. The complex eigenvalues of $\underset{\tilde{\mathcal{H}}}{\mathrm{eff}},\left\{E_{j}-\frac{i}{2} \Gamma_{j}\right\}$, give the energies $\left\{E_{j}\right\}$ and decay rates $\left\{\Gamma_{j}\right\}$ of the individual quantum (metastable) states of the reactant molecule provided they are non-overlapping, i.e.,

$$
\Gamma_{j}<\left|E_{j}-E_{j^{\prime}}\right|
$$

(Alternatively, $\left\{E_{j}\right\}$ and $\left\{\Gamma_{j}\right\}$ are the positions and widths of scattering resonances between bimolecular collisions of the fragment molecules.) In light of Eq. (5.3), it is reasonable to treat the imaginary part of ${\underset{\mathcal{F}}{\mathcal{H}}}^{\text {eff }}$ by first order perturbation theory: the "zeroth order" eigenstates,

$$
\left|\Psi_{j}\right\rangle=\sum_{m}|m\rangle c_{m, j}
$$

are thus the eigenvectors of the real Hamiltonian matrix, $\underset{\sim}{\mathcal{H}}$, and the imaginary parts of the eigenvalues are given (within first order perturbation theory) by

$$
\begin{aligned}
\Gamma_{j .} & \equiv\left\langle\Psi_{j}|\Gamma| \Psi_{j}\right\rangle \\
& =\sum_{m, m^{\prime}} c_{m, j} \Gamma_{m, m^{\prime}} c_{m^{\prime}, j} \\
& =c_{j}^{T} \cdot \underset{\sim}{\Gamma} c_{j} .
\end{aligned}
$$

The unimolecular decay rate for a state characterized by eigenvector $c$ is therefore $\Gamma(\underline{c})=\underline{c}^{T} \cdot \underset{z}{\Gamma} \cdot \underset{\sim}{c}$

One next assumes that the eigenstates of $\underset{\tilde{\mathcal{H}}}{\mathcal{H}}$ are strongly mixed, i.e., that the dynamics of the (highly excited) reactant molecule is chaotic, ergodic, etc. This corresponds to assuming that the statistics of the eigenstates within a given narrow energy interval are equivalent to sampling over all states consistent with normalization, $c^{T} \cdot c=1$. Physically, this uniform sampling will involve a random mixture of only a finite number of basis states since random coupling of an infinite basis would entail the loss of energy information. Thus the "zeroth order state" reduces to the generic form,

$$
\left|\Psi_{j}\right\rangle=\sum_{m=1}^{f}|m\rangle c_{m, j}^{\prime}
$$


where $f$ is the effective number of strongly mixed states in a generic basis. (Sec. 5.4.2 discusses more fully the physical significance of the parameter, $f$.) Thus the probability distribution of the decay rate may be written as

$$
P(\Gamma)=\int_{-\infty}^{\infty} \cdots \int_{-\infty}^{\infty} d c_{1}^{\prime} \cdots d c_{f}^{\prime} P_{f}^{\prime}\left(c_{c}^{\prime}\right) \delta\left(\Gamma-\underline{c}^{\prime T} \cdot \underset{\sim}{\Gamma} \cdot c^{\prime}\right)
$$

where the probability of a given state is

$$
P_{f}^{\prime}\left(c^{\prime}\right) \equiv \delta\left(c^{\prime T} \cdot c^{\prime}-1\right)
$$

which enforces the uniform sampling of the normalized states.

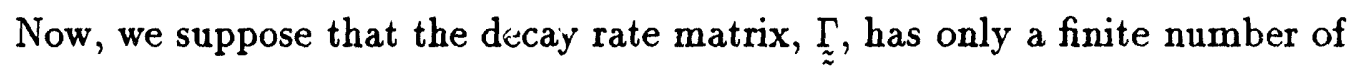
non-zero eigenvalues, $n$. A transformation of the state vectors, $c^{\prime}$, to a space in which the first $n$ basis states span the same space as that spanned by those eigenvectors of $\Gamma_{\tilde{z}}$ with nonzero eigenvalues, reduces Eq. (5.7) to

$$
P(\Gamma)=\int_{-\infty}^{\infty} \cdots \int_{-\infty}^{\infty} d c_{1} \cdots d c_{n} P_{f, n}(c) \delta\left(\Gamma-c^{T} \cdot \underset{\tilde{z}}{\Gamma} \cdot c\right)
$$

where $c$ is an $n$-dimensional vector and

$$
P_{f, n}(c)=\int_{-\infty}^{\infty} \ldots \int_{-\infty}^{\infty} d c_{n+1} \cdots d c_{f} P_{f}\left(c_{1}, \ldots, c_{n}, c_{n+1}, \ldots, c_{f}\right) .
$$

As shown by Ullah ${ }^{150}$, the probability distribution for the vector $c$ can be readily integrated to obtain,

$$
P_{f, n}(c)=\pi^{-1 / 2} \frac{G(f / 2)}{G\left(\frac{f-n}{2}\right)}\left(1-\sum_{i=i}^{n} c_{i}^{2}\right)^{(f-n-2) / 2}
$$

where $G$ is the gamma function ${ }^{60}$ which is normally denoted $\Gamma$, but here we need to avoid confusion with our notation for the decay rate $\Gamma$. Moreover, in the large $f$ limit, the asymptotic result is:

$$
P_{f, n}(c)=\prod_{j=1}^{n} \sqrt{\frac{f}{2 \pi}} \mathrm{e}^{-f c_{j}^{2} / 2}
$$

as can be anticipated ${ }^{77}$ from the central limit theorem. Although in what follows we shall assume that $f$ is large and consequently use the asymptotic form [Eq. (5.12)] in 
Eq. (5.9) to obtain the decay rate probability distribution, it should be noted that this assumption can be relaxed. However, since this general result for $P_{f}^{\prime}$ is not separable with respect to each of the coordinates of $\underline{c}^{\prime}$, its multidimensional integral can not be reduced further, and is thus not amenable to applications.

\subsubsection{The Decay Rate Probability Distribution}

Using the results from the previous subsection, the decay rate probability distribution may be written as:

$$
P(\Gamma)=\int_{-\infty}^{\infty} \cdots \int_{-\infty}^{\infty} d c_{1} \cdots d c_{n} \prod_{j=1}^{n} \sqrt{\frac{f}{2 \pi}} e^{-f c_{j}^{2} / 2} \delta\left(\Gamma-c^{T} \cdot \underset{\tilde{z}}{\Gamma} \cdot \underset{\sim}{c}\right)
$$

in the asymptotic limit where $f$ is large. Introducing the integral form of Dirac's $\delta$-function, $\delta(z)=(2 \pi)^{-1} \int_{-\infty}^{\infty} \mathrm{e}^{i z t} d t$, and changing the order of integration,

$$
P(\Gamma)=\int_{-\infty}^{\infty} d t e^{i \Gamma t}\left(\frac{f}{2 \pi}\right)^{n / 2} \int_{-\infty}^{\infty} \cdots \int_{-\infty}^{\infty} d c_{1} \cdots d c_{n} e^{-f \underline{c} \underline{c}^{T} \cdot{ }^{\prime} / 2} e^{\underline{c}^{\prime T} \cdot \Gamma \cdot c^{\prime}}
$$

The Gaussian integrals in this last equation can be readily evaluated to provide

$$
P(\Gamma)=(2 \pi)^{-1} \int_{-\infty}^{\infty} d t \mathrm{e}^{i \Gamma t} D^{\prime}(t)^{-1 / 2}
$$

where

$$
\begin{aligned}
D^{\prime}(t) & =\operatorname{det}[\underset{\tilde{z}}{I}+2 i t \underset{\tilde{z}}{\Gamma} / f] \\
& =\prod_{j=1}^{n}\left(1+2 i t \gamma_{j} / f\right),
\end{aligned}
$$

and $\left\{\gamma_{j}\right\}$ are the $n$ non-zero eigenvalues of the $\underset{\tilde{\sim}}{m}$ matrix. (Note that, in general $n \ll f$, i.e. most of these eigenvalues are zero.)

It is useful to define the dimensionless reduced probability distribution in terms of $P(\Gamma)$ as

$$
p(x) \equiv \bar{\Gamma} P(\bar{\Gamma} x)
$$


where $x=\Gamma / \bar{\Gamma}$ is the decay rate in units of the average rate $\bar{\Gamma}$. From Eq. (5.15), and making use of the calculated value of $\bar{\Gamma}$ in Eq. (5.24) obtained below, one sees that

$$
p(x)=(2 \pi)^{-1} \int_{-\infty}^{\infty} d t \mathrm{e}^{i x t} D(t)^{-1 / 2}
$$

where

$$
\begin{aligned}
D(t) & =\operatorname{det}\left[{\underset{\tilde{z}}{I}}^{I}+2 i t \underset{\tilde{\tau}}{\Gamma} / \operatorname{tr}(\underset{\tilde{z}}{\Gamma})\right] \\
& =\prod_{j=1}^{n}\left(1+2 i t \gamma_{j} / \operatorname{tr}(\underset{\tilde{z}}{\Gamma})\right) .
\end{aligned}
$$

If there are exactly $n$ equal non-zero eigenvalues, i.e. $\gamma_{j}=\gamma$ for $j \leq n$, Eq. (5.17) reduces to a $\chi^{2}$-distribution with $n$ degrees of freedom - the PorterThomas result - and this has also been the traditional model used to describe fluctuations in the unimolecular decay rate of chemical reactions. ${ }^{151,152}$ Various simplified but nontrivial models for the $\left\{\gamma_{j}\right\}$ beyond this approximation will be explored in Sec. 5.3. In the most general case one can obtain the distribution from Eq. (5.17) by numerical integration provided, of course, that one has the eigenvalues $\left\{\gamma_{j}\right\}$ of $\underset{\tilde{z}}{\Gamma}$. Note that these expressions are written as Fourier transforms in order to suggest the use of an FFT numerical procedure for their evaluation. If instead a direct integration is desired then these expressions can be written in a manifestly real form; see Eqs. (3.1) and (3.2) in Ref. 100.

\subsubsection{The Microcanonical Quantum Survival Probability}

The "microcanonical quantum survival probability" 12 is defined as a sum over a continuum of states with exponential decay, e.g.,

$$
P(t)=\int_{0}^{\infty} d \Gamma P(\Gamma) \mathrm{e}^{-\Gamma t}
$$

This would correspond to the survival probability of a broad band excitation or collision experiment in which a dense set of exponentially decaying excited states are prepared uniformly in a narrow energy interval. $\mathrm{Miller}^{151}$ and $\mathrm{Hase}^{152}$ have considered 
this quantity explicitly for the case of Porter-Thomas distributions for $P(\Gamma)$. With the more general result for $P(\Gamma)$ given by RM/TST, Eq. (5.57) above, it is relatively straightforward to obtain the following result for $P(t)$,

$$
P(t)=\prod_{j}\left(1+2 t \bar{\Gamma} \gamma_{j} / \operatorname{tr}(\underset{\tilde{\tau}}{\Gamma})\right)^{-1 / 2}
$$

If all the $\gamma_{j}$ 's are the same, then Eq. (5.19) reduces to that given previously ${ }^{151}$ for a $\chi^{2}$ distribution.

Further progress in elucidating the result in Eq. (5.19) can be made by anticipating the result to be described in Sec. 5.4. The result is that

$$
\gamma_{j}=\epsilon N_{\underline{n}}
$$

where $j$ is a collective index in one-to-one correspondence with $\underset{\sim}{n}$ which labels the states of the activated complex, $N_{n}$ is the transmission probability through a given state of the activated complex, and $\epsilon$ is a constant of proportionality. Thus Eq. (5.19) can be written as

$$
P(t)=\prod_{\underline{n}}\left(1+\frac{N_{n} t}{\pi \rho}\right)^{-1 / 2} .
$$

In the limit that $t \rightarrow 0$, this equation reduces to,

$$
P(t) \approx \prod_{n}\left(1-\frac{1}{2} \frac{N_{n} t}{\pi \rho}\right) \approx\left(1-\sum_{n} \frac{N_{n} t}{\pi \rho}\right) \approx 1-\bar{\Gamma} t \approx \mathrm{e}^{-\bar{\Gamma} t},
$$

which demonstrates in the short time limit the exponential fall-off of the survival probability as determined by the average rate.

\subsubsection{The Effective Number of Channels: $\nu_{\text {eff }}$}

As discussed in Sec. 1.2, it has been common practice to use the $\chi^{2}$ family of distributions to fit to experimental data. The single parameter $\nu$ which uniquely determines a member of this family has traditionally been determined from either the second moment of the experimental data or from the expectation value of the logarithm of the rate as motivated by the maximum-likelihood method. 
Although the RM/TST family of distributions do contain the family of $\chi^{2}$ distributions, they are not equal and consequently $\nu_{\text {eff }}$ does not uniquely specify a given member of the RM/TST family of distributions, as is illustrated in Sec. 5.3. Nonetheless one can associate a value of $\nu_{e f f}$ to each of the RM/TST distributions as one does for observed distributions. In this section, we obtain analytic results for the value of $\nu_{\text {ef } f}$ as obtained using the two most commonly imposed constraints discussed above.

\section{$\nu_{\text {eff }}^{0}$ Using Moments}

Recall that the effective number of channels may be written in terms of the first and second moments of the decay rates as,

$$
\nu_{e f f}^{0} \equiv \frac{2\langle\Gamma\rangle^{2}}{\left\langle\Gamma^{2}\right\rangle-\langle\Gamma\rangle^{2}} .
$$

We now proceed to calculate these moments.

To obtain the RM/TST moments, it is actually most convenient to use the form of the probability distribution in Eq. (5.13), from which it follows that:

$$
\begin{aligned}
\left\langle\Gamma^{n}\right\rangle & \equiv \int_{-\infty}^{\infty} d \Gamma \Gamma^{n} P(\Gamma) \\
& =\int_{-\infty}^{\infty} d \Gamma \Gamma^{n} \int_{-\infty}^{\infty} \cdots \int_{-\infty}^{\infty} d c_{1} \cdots d c_{n} \prod_{j=1}^{n} \sqrt{\frac{f}{2 \pi}} e^{-f c_{j}^{2} / 2} \delta\left(\Gamma-c^{T} \cdot \underset{\tilde{z}}{T} \cdot c\right) \\
& =\int_{-\infty}^{\infty} \cdots \int_{-\infty}^{\infty} d c_{1} \cdots d c_{n} \prod_{j=1}^{n} \sqrt{\frac{f}{2 \pi}} e^{-f c_{j}^{2} / 2}\left(\sum_{j=1}^{n} \gamma_{j} c_{j}^{2}\right)^{n}
\end{aligned}
$$

where $\left\{\gamma_{i}\right\}$ are the eigenvalues of $\underset{\tilde{c}}{\Gamma}$ and an orthogonal transformation of the integration variables has been effected. The first moment reduces readily into a sum of Gaussian integrals:

$$
\begin{aligned}
\bar{\Gamma} \equiv\langle\Gamma\rangle & =\sum_{j=1}^{f} \int_{-\infty}^{\infty} d c_{j} \gamma_{j} c_{j}^{2} \sqrt{\frac{f}{2 \pi}} e^{-f c_{j}^{2} / 2} \\
& \left.=\frac{1}{f} \sum_{j=1}^{f} \gamma_{j}=\frac{1}{f} \operatorname{tr} \underset{\sim}{\Gamma}\right) .
\end{aligned}
$$


[Note that this determines the effective number of mixed states as

$$
\left.f=\frac{\operatorname{tr}(\underset{\tilde{\Sigma}}{\Gamma})}{\bar{\Gamma}} .\right]
$$

The second moment is only slightly more involved:

$$
\begin{aligned}
\left\langle\Gamma^{2}\right\rangle & =\sum_{j=1}^{f} \int_{-\infty}^{\infty} d c_{j} \gamma_{j}^{2} c_{j}^{4} \sqrt{\frac{f}{2 \pi}} e^{-f c_{j}^{2} / 2}+\sum_{i \neq j}^{f} \gamma_{i} \gamma_{j}\left(\int_{-\infty}^{\infty} d c c^{2} \sqrt{\frac{f}{2 \pi}} e^{-f c^{2} / 2}\right) \\
& =\frac{3}{f^{2}} \sum_{j=1}^{f} \gamma_{j}^{2}+\frac{1}{f^{2}} \sum_{i \neq j}^{f} \gamma_{i} \gamma_{j},
\end{aligned}
$$

and the variance of the distribution takes on the simple form:

$$
\left\langle\Gamma^{2}\right\rangle-\langle\Gamma\rangle^{2}=\frac{2}{f^{2}} \sum_{j=1}^{n} \gamma_{j}^{2}=\frac{2}{f^{2}} \operatorname{tr}\left({\underset{\sim}{\Gamma}}^{2}\right)
$$

Combining Eqs. (5.24) and (5.27), the effective number of channels for a given RM/TST distribution can be written directly in terms of the $\Gamma$ matrix or its eigenvalues,

$$
\nu_{\text {eff }}^{0}=\frac{\left(\sum \gamma_{j}\right)^{2}}{\sum \gamma_{j}^{2}}=\frac{\operatorname{tr}(\underset{\tilde{\tau}}{\Gamma})^{2}}{\operatorname{tr}\left({\underset{\tilde{z}}{2}}^{2}\right)}
$$

and this provides an analytic value of $\nu_{\text {eff }}^{0}$ if the eigenvalues of $\underset{\tilde{\sim}}{\Gamma}$ are known.

\section{$\nu_{e f f}^{M L}$ Using the Maximum-Likelihood Method}

Within the maximum-likelihood formalism, ${ }^{68}$ the effective number of channels is computed by inverting the transcendental equation,

$$
F(z) \equiv G(z) \frac{\partial G(z)}{\partial z}-\ln z
$$

for $z=\nu_{e f f}^{M L} / 2$, where $G$ is the gamma function ${ }^{60}$ and $F\left(\nu_{e f f}^{M L} / 2\right)$ is computed directly from the data,

$$
F\left(\nu_{e f f}^{M L} / 2\right)=\frac{1}{p} \sum_{i=1}^{p} \ln \left(\Gamma_{i} / \bar{\Gamma}\right) .
$$

Given a continuous distribution, this result takes its continuous form, e.g.,

$$
F\left(\nu_{e f f}^{M L} / 2\right)=\langle\ln (\Gamma / \bar{\Gamma})\rangle=\langle\ln x\rangle
$$


where the averages in this equation are taken with respect to the RM/TST distributions in Eqs. (5.15) and (5.17), respectively. The calculation of $F\left(\nu_{e f f}^{M L} / 2\right)$ for the RM/TST distributions, in the most general case of the eigenvalues of $\underset{\tilde{z}}{\Gamma}$, can be readily computed numerically with a standard integrator. In this section, analytical expressions for $\langle\ln x\rangle$ are derived for various special cases.

Using the reduced probability distribution, Eq. (5.17), the expectation value of the logarithm is

$$
\begin{aligned}
\langle\ln x\rangle & =\int_{0}^{\infty} d x \ln \mathrm{x} \frac{1}{2 \pi} \int_{-\infty}^{\infty} d t \mathrm{e}^{i x t} D(t)^{-1 / 2} \\
& =\int_{-\infty}^{\infty} d t D(t)^{-1 / 2} \frac{1}{2 \pi} \int_{0}^{\infty} d t \mathrm{e}^{i x t} \ln \mathrm{x}
\end{aligned}
$$

where $D(t)$ is defined by Eq. (5.17b). If the limits are taken carefully, the indefinite integral over $x$ can be evaluated by an integration by parts, resulting in

$$
\langle\ln x\rangle=\lim _{\epsilon \rightarrow 0}\left\{\frac{1}{2 \pi i} \int_{-\infty+i \epsilon}^{\infty+i \epsilon} d t D(t)^{-1 / 2}\left[\gamma_{e}+\ln (-i t)\right]\right\}
$$

where $\gamma_{e}$ is Euler's constant. ${ }^{60}$ The $\gamma_{e}$ term in this integral can be evaluated by closing the contour on the lower half plane, using the residue theorem ${ }^{134}$ for the only pole contained within it at $t=0$, and noting that $D(0)=1$; the result is:

$$
\begin{aligned}
\langle\ln x\rangle & =-\gamma_{e}+\lim _{\epsilon \rightarrow 0}\left[\frac{1}{2 \pi i} \int_{-\infty+i \epsilon}^{\infty+i \epsilon} d t D(t)^{-1 / 2} \ln (-i t)\right] \\
& =-\gamma_{e}+\lim _{\epsilon \rightarrow 0}\left\{\frac{1}{2 \pi i} \int_{-\infty+i \epsilon}^{\infty+i \epsilon} d t \ln (-i t) \prod_{j=1}^{n}\left[1+2 i t \bar{\gamma}_{j}\right]^{-1 / 2}\right\},
\end{aligned}
$$

where $D(t)$ has now been written explicitly, and the normalized eigenvalues are defined by

$$
\bar{\gamma}_{j} \equiv \gamma_{j} / \operatorname{tr}(\underset{\tilde{z}}{\Gamma})
$$

If each of the eigenvalues of $\underset{\tilde{x}}{\Gamma}$ is precisely doubly degenerate, then Eq. (5.34) can be evaluated by closing the contour on the upper half plane and making use of 
the residue theorem for each of the poles associated with each degenerate eigenvalue; the result is:

$$
\langle\ln x\rangle=-\gamma_{e}+\sum_{k=1}^{n / 2}\left[\ln \left(2 \bar{\gamma}_{2 k}\right) \prod_{l=1}^{n / 2^{\prime}}\left(1-\bar{\gamma}_{2 l} / \bar{\gamma}_{2 k}\right)^{-1}\right],
$$

where the prime on the product refers to the exclusion of $l=k$, and the eigenvalues $\left\{\bar{\gamma}_{j}\right\}$ are assumed to be ordered from largest to smallest.

Alternatively, if each of eigenvalues of $\underset{\tilde{z}}{\Gamma}$ is non-degenerate, then Eq. can be written as a sum of one-dimensional integrals,

$$
\langle\ln x\rangle=-\gamma_{e}+\frac{1}{2 \pi} \sum_{k=1}^{n / 2} \int_{2 \bar{\gamma}_{2 k}}^{2 \bar{\gamma}_{(2 k-1)}} d t \frac{\ln t}{\left(2 \bar{\gamma}_{(2 k-1)}-t\right)^{1 / 2}\left(t-2 \bar{\gamma}_{2 k}\right)^{1 / 2}} \prod_{j}^{\prime \prime}\left(1-2 \bar{\gamma}_{j} / t\right)^{-1 / 2}
$$

where the double prime on the product refers to the exclusion of $j=2 k$ and $j=$ $(2 k-1)$. Note that some of the square root terms within the product are complex and are evaluated properly using the plus sign convention for all complex square root function evaluations. If the $\left\{\gamma_{j}\right\}$ are sufficiently separated, then this can be approximately computed taking the product to be slowly varying and evaluated at an effective value of $t$, i.e.,

$$
\begin{aligned}
\langle\ln x\rangle & \approx-\gamma_{e}+\frac{1}{2 \pi} \sum_{k=1}^{n / 2}\left[\prod_{j}^{\prime \prime}\left(1-2 \bar{\gamma}_{j} / t_{k}^{*}\right)^{-1 / 2}\right] \int_{2 \gamma_{2 k}}^{2 \gamma_{2 k-1}} d t \frac{\ln t}{\left(2 \gamma_{(2 k-1)}-t\right)^{1 / 2}\left(t-2 \gamma_{2 k}\right)^{1 / 2}} \\
& \approx-\gamma_{e}+\sum_{k=1}^{n / 2}\left[\ln \left(2 t_{k}^{*}\right) \prod_{j}^{\prime \prime}\left(1-\bar{\gamma}_{j} / t_{k}^{*}\right)^{-1 / 2}\right]
\end{aligned}
$$

where the remaining integral is evaluated using Eq. 4.293.13 in Gradshteyn and Ryzhik ${ }^{153}$, and

$$
t_{k}^{*}=\frac{1}{4}\left[\sqrt{\bar{\gamma}_{(2 k-1)}}+\sqrt{\bar{\gamma}_{2 k}}\right]^{2}
$$

is chosen so that the result reduces to Eq. (5.36) in the limit that the eigenvalues are doubly degenerate.

In order to discuss the relative accuracy of implementing these expressions, the results for the $\mathrm{D}_{2} \mathrm{CO}$ dissociation to be described in Chapter 6 need be anticipated. In all cases except for case (a) in which the degenerate eigenvalues corresponding to $\pm|K|$ are included in the same symmetry manifold, Eq. (5.38) provides 
accurate results compared to the direct computation of $\langle\ln x\rangle$. However, the approximate result, Eq. (5.39), provides qualitative agreement only for case (c), in which the eigenvalues were well-separated. In practice, both the exact result, Eq. (5.38), and the direct computation of $\langle\ln x\rangle$ were performed as a further chec's of the code. Inversion of Eq. (5.31) subsequently provides the effective number of channels within the maximum-likelihood formalism.

\subsection{Model Calculations}

Before converting the model just developed into a predictive one by evaluating the eigenvalues $\left\{\gamma_{j}\right\}$ of the decay rate matrix $\underset{\tilde{z}}{\Gamma}$ (up to a proportionality factor), it is useful to first exhibit some possible distributions and compare them to $\chi^{2}$-distributions.

\subsubsection{The Porter-Thomas Limit}

An adiabatic picture of the dissociation process suggests that the vibrational quantum numbers corresponding to the modes orthogonal to the reaction path coordinate will remain approximately conserved throughout the dynamics. The eigenvalues $\left\{\gamma_{j}\right\}$ of $\underset{\approx}{\Gamma}$ will thus correspond to the decay rates of an effective one-dimensional Hamiltonian where the collective index $j$ corresponds to the adiabatic quantum numbers and labels the "channels" through the barrier. If the reactions were classical or the intramolecular energy redistribution (IVR) between the perpendicular modes were fast compared to the motion along the reaction coordinate, then the non-zero eigenvalues of $\underset{\tilde{z}}{ }$ are equal and all of the eigenvalues may be described by a step function,

$$
\gamma_{j}= \begin{cases}\gamma, & \text { if } j \in\{1, \ldots, \nu\} ; \\ 0, & \text { if } j>\nu,\end{cases}
$$

with $\nu$ non-zero channels. (Note that the actual rate of the fastest mode is irrelevant, as this scales away in the reduced probability distribution, Eq. (5.17a), which is to be obtained.) As mentioned earlier, with this form of the eigenvalues the distribution obtained in the previous section reduces to a $\chi^{2}$-distribution with $\nu$ degrees of 


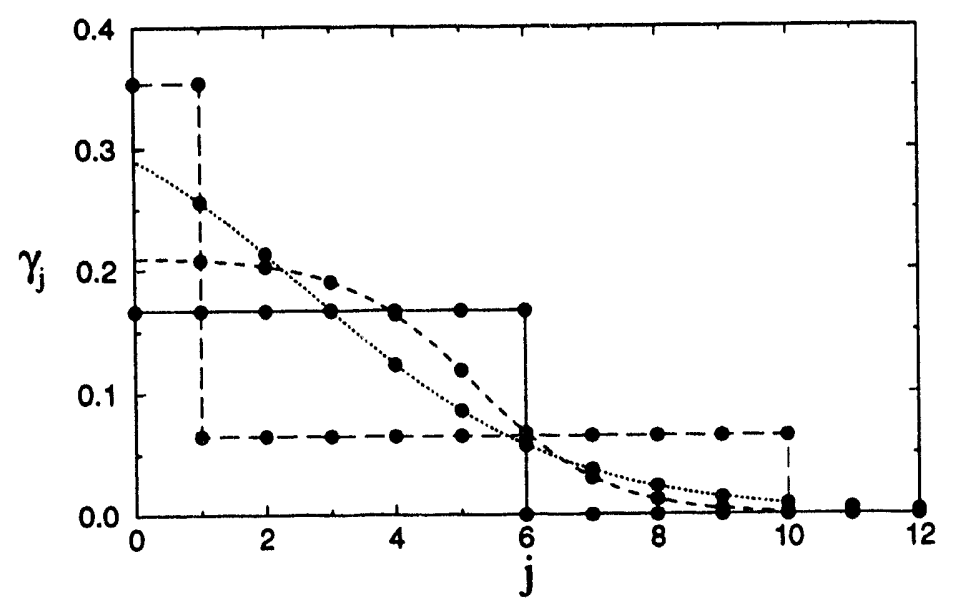

Figure 5.2: Characteristic functional forms for $\left\{\gamma_{j}\right\}$ with $\nu_{\text {eff }}=6$ compared to the step-function form which leads to a $\chi^{2}$-distribution. The short dashed and medium dashed curves correspond to the smooth step function form with $\left\{\alpha=.5, \nu_{0}=2.109\right\}$ and $\left\{\alpha=1.0, \nu_{0}=4.738\right\}$; the distributions obtained with these models are presented in Fig. 5.3. The long dashed curve is a bi-step function with $\left\{\nu_{0}=1, \nu_{1}=10\right\}$ and the distribution it generates is presented in Fig. 5.5. Note also that all curves have been renormalized so that the $\left\{\gamma_{j}\right\}$ sum to one.

freedom.

\subsubsection{Smooth Step Function Model}

A non-trivial modification to the classical picture just described can be obtained by assuming that the eigenvalues $\gamma_{j}$ are described by a smooth step function. This is tantamount to assuming that the system exhibits tunneling but that the activated complex has little or no quantization. The explicit form of the smooth step function is

$$
\gamma_{j} \propto\left[1+e^{\alpha\left(j-\nu_{0}-\frac{1}{2}\right)}\right]^{-1},
$$

for each integer index $j$, and with $\alpha$ and $\nu_{0}$ parameters characterizing the assumed form. Since distributions are classified using $\nu_{\text {eff }}, \nu_{0}$ is chosen for a given value of $\alpha$ such that the variance criterion, Eq. (5.27), is satisfied. (Smooth step functions obtained using $\alpha$ 's characteristic of those studied and with $\nu_{\text {eff }}=6$ are compared to a step function with $\nu=6$ non-zero $\gamma$ 's in Fig. 5.2.) Note that in the limit $\alpha \rightarrow \infty$, 


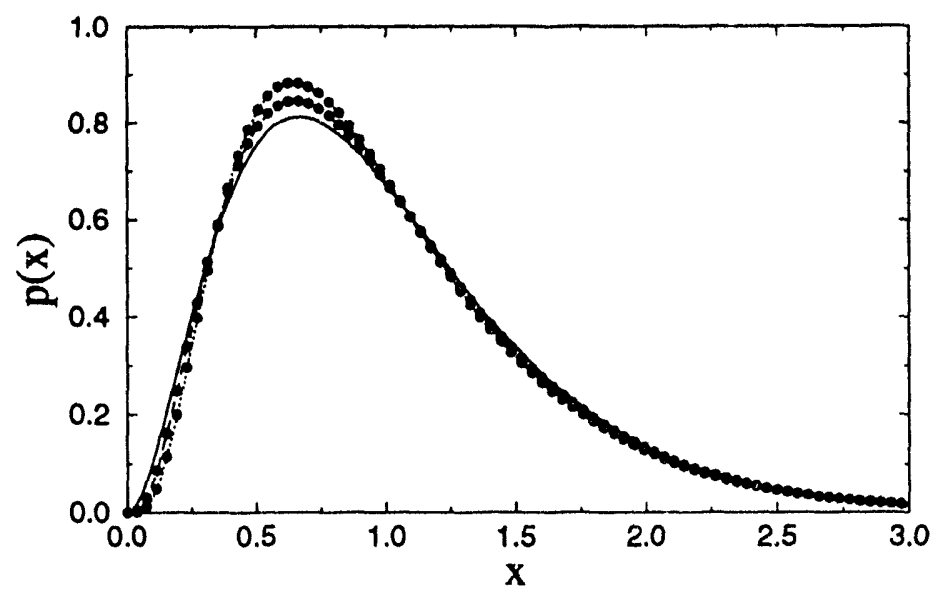

Figure 5.3: The reduced probability distributions [cf. Eq. (5.17)] with $\nu_{\text {eff }}=6$ obtained using the smooth step function model. The solid curve is the $\chi^{2}$-distribution, and the short dashed and long dashed curves correspond to the smooth step function form with $\left\{\alpha=.5, \nu_{0}=2.109\right\}$ and $\left\{\alpha=1.0, \nu_{0}=4.738\right\}$, respectively.

Eq. (5.41) reverts to the step function defined in Eq. (5 40) with $\nu_{0}=\nu\left(=\nu_{\text {eff }}\right.$ ) thereby reproducing the $\chi^{2}$-distribution; with finite values of $\alpha$ - as illustrated in Fig. 5.2 - the fall-off of the $\gamma_{j}$ 's is more gradual and this leads to deviations from the $\chi^{2}$-distribution.

Although the distributions for various values of $\nu_{\text {ef } f}$ have been explored, only the distributions with $\nu_{\text {eff }}=6$ and 2 are presented in Figs. 5.3 and 5.4, respectively, because these cases illustrate the behavior seen in general. As can be seen, the models deviate only slightly from the $\chi^{2}$-distribution with respect to all features except for the small rate behavior in the $\nu_{\text {eff }}=2$ case. Unfortunately, as will be elaborated later, experimental results often miss many of the small rates and this region would not be useful in differentiating between candidate distributions. Thus the markedly different behavior from the $\chi^{2}$-distributions seen in the experimental results for the formaldehyde dissociation could not be accounted for by this not-quiteclassical model. 


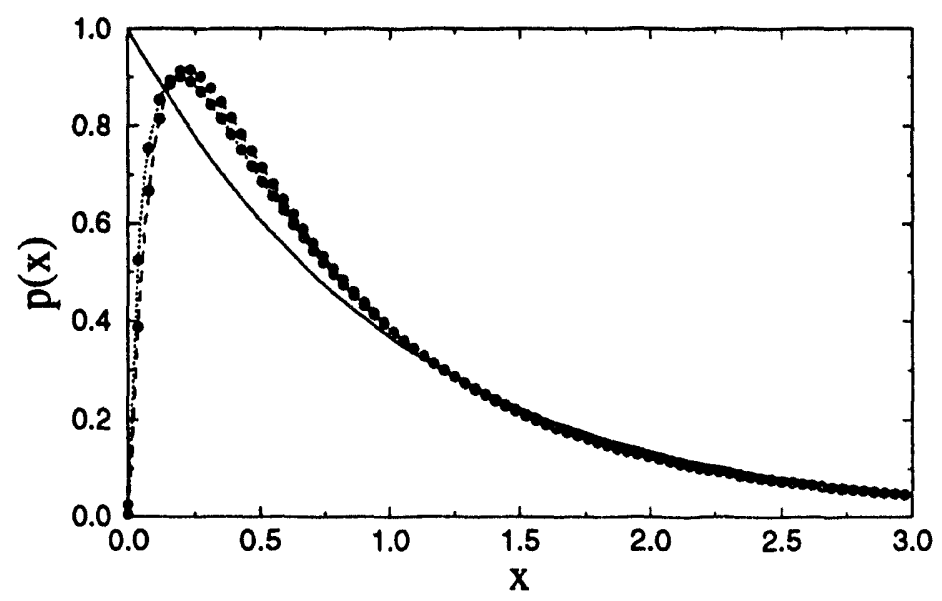

Figure 5.4: Same as Fig. 5.3 except that $\nu_{\text {eff }}=2$ and $\left\{\alpha=1.25, \nu_{0}=-.291\right\}$ and $\left\{\alpha=1.75, \nu_{0}=1.0173\right\}$, respectively.

\subsubsection{Bi-Step Function Model}

An alternate model describing the eigenvalues of the decay rate matrix is motivated by considering the quantization of the activated complex. Within the vibrational adiabatic approximation ${ }^{17,19,20}$ the multidimensional potential is assumed to be separable and the perpendicular modes are taken to be harmonic, e.g.,

$$
V(s)=V_{0}(s)+\sum_{k=1}^{F-1} \omega_{k}\left(n_{k}+\frac{1}{2}\right)
$$

where $s$ is the reactior coordinate, $\omega_{i}$ is the frequency of the perpendicular mode $i$ and $V_{0}(s)$ is the effective one-dimensional potential along $s$. Within this approximation, the eigenvalues of the multidimensional decay rate matrix are related to the onedimensional decay rate, $\gamma_{1 \mathrm{D}}(E)$, for the potential, $V_{0}(s)$ :

$$
\gamma_{n}=\gamma_{1 \mathrm{D}}\left(E_{T}-\sum_{i=1}^{F-1} \hbar \omega_{i}\left(n_{i}+\frac{1}{2}\right)\right)
$$

where $E_{T}$ is the total energy. In the limit that $\hbar$ is small, $\gamma_{1 \mathrm{D}}(E)$ is monotonically increasing with $E$, and there is a simple ordering of the eigenvalues of the decay rate matrix as determined by the number of quanta in the perpendicular modes:

$$
\gamma_{\underline{n}} \geq \gamma_{\underline{n}^{\prime}} \Longleftrightarrow \sum_{k=1}^{F-1} \hbar \omega_{i}\left(n_{i}+\frac{1}{2}\right) \leq \sum_{k=1}^{F-1} \hbar \omega_{i}\left(n_{i}^{\prime}+\frac{1}{2}\right)
$$


(This representation can be improved, and this is the subject of the discussion in Sec. 5.4.1.) If one further assumes that the perpendicular mode frequencies are (approximately) equal, $\omega_{i} \approx \omega$, then there will be a single large eigenvalue of the decay rate matrix corresponding to no quanta in the perpendicular modes - the "ground state" of the activated complex. The next largest eigenvalue is $(F-1)$ degenerate and corresponds to those states with a single quanta in only one of the perpendicular modes - the "fundamentals" of the activated complex. This suggests an approximate model of the decay process in which there is fast decay through a single channel, there is slow decay through a degenerate set of channels, and the remaining decay which is even smaller - is ignored.

A slight generalization of this model, permitting the degeneracy of the fast channel, may be described by the bi-step function,

$$
\gamma_{j}= \begin{cases}\gamma, & \text { if } j \in\left\{1, \ldots, \nu_{0}\right\} ; \\ \lambda \gamma, & \text { if } j \in\left\{\nu_{0}+1, \ldots, \nu_{0}+\nu_{1}\right\} \\ 0, & \text { if } j>\nu_{0}+\nu_{1},\end{cases}
$$

with $\nu_{0}$ fast channels and $\nu_{1}$ slow channels. For this model, Eq. (5.27) yields,

$$
\nu_{e f f}=\left(\nu_{0}+\lambda \nu_{1}\right)^{2} /\left(\nu_{0}+\lambda^{2} \nu_{1}\right)
$$

which varies from $\nu_{0}$ to $\nu_{0}+\nu_{1}$ as $\lambda$ varies from 0 to 1 . The reduced probability distribution, Eq. (5.17a), is

$$
p(x)=(2 \pi)^{-1} \int_{-\infty}^{\infty} d t \mathrm{e}^{i x t}(1+2 i t / S)^{-\nu_{0} / 2}(1+2 i t \lambda / S)^{-\nu_{1} / 2},
$$

with

$$
S \equiv \nu_{0}+\lambda \nu_{1}
$$

and can be evaluated analytically yielding (see, for example, Eq. 3.384.7 in Gradshteyn and Ryzhik ${ }^{153}$ )

$$
p(x)=\frac{(S / 2)(x S / 2)^{\left(\nu_{0}+\nu_{1}\right) / 2-1} e^{-x S / 2 \lambda}}{\lambda^{\nu_{1} / 2} G\left[\left(\nu_{0}+\nu_{1}\right) / 2\right]}{ }_{1} F_{1}\left[\frac{\nu_{0}}{2} ; \frac{\nu_{0}+\nu_{1}}{2} ; \frac{S}{2}\left(\frac{1}{\lambda}-1\right) x\right]
$$




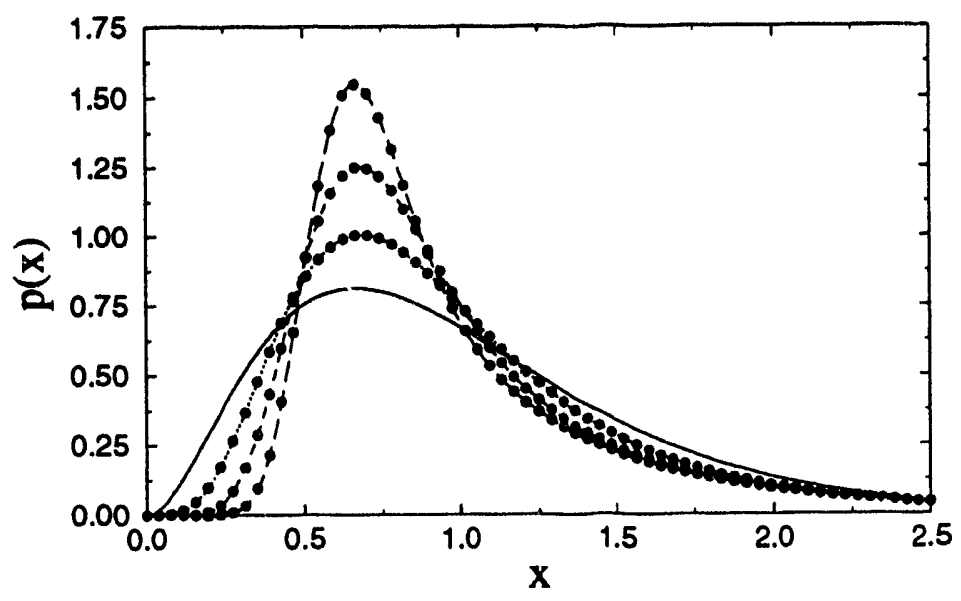

Figure 5.5. The reduced probability distributions [cf. Eq. (5.17)] with $\nu_{\text {eff }}=6$ obtained using the bi-step function model. The solid curve is the $\chi^{2}$-distribution, and the short, medium and long dashed curves correspond to the smooth step function form with $\left\{\nu_{1}=10, \lambda=.183\right\},\left\{\nu_{1}=20, \lambda=.0801\right\}$ and $\left\{\nu_{1}=40, \lambda=.0380\right\}$, respectively.

where ${ }_{1} F_{1}$ is the hypergeometric function, which can be simply related to a confluent hypergeometric function, ${ }^{60}$ and $G$ is the gamma function. In the special case that $\nu_{0}=\nu_{1}=1$, this reduces to the result derived by Polik et al., ${ }^{98}$

$$
p(x)=\frac{(1+\lambda)}{2 \lambda^{1 / 2}} e^{-x(1+\lambda)^{2} / 4 \lambda} I_{0}\left[x\left(1-\lambda^{2}\right) / 4 \lambda\right],
$$

where $I_{0}$ is the Bessel function of imaginary argument.

Returning now to the more common physically relevant cases with $\nu_{0}=$ 1, Figs. 5.5 and 5.6 exhibit the dramatic deviations from the corresponding $\chi^{2}-$ distributions that this model affords. The deviations certainly increase with the number of slow channels, $\nu_{1}$. In a chemical system, the slow channels will not be degenerate and the deviations will not be as dramatic. Nonetheless, these deviations do persist for chemical systems as presented in the next section in which the eigenvalues, up to a proportionality constant, are evaluated using the semiclassical transition state theory described in earlier chapters. 


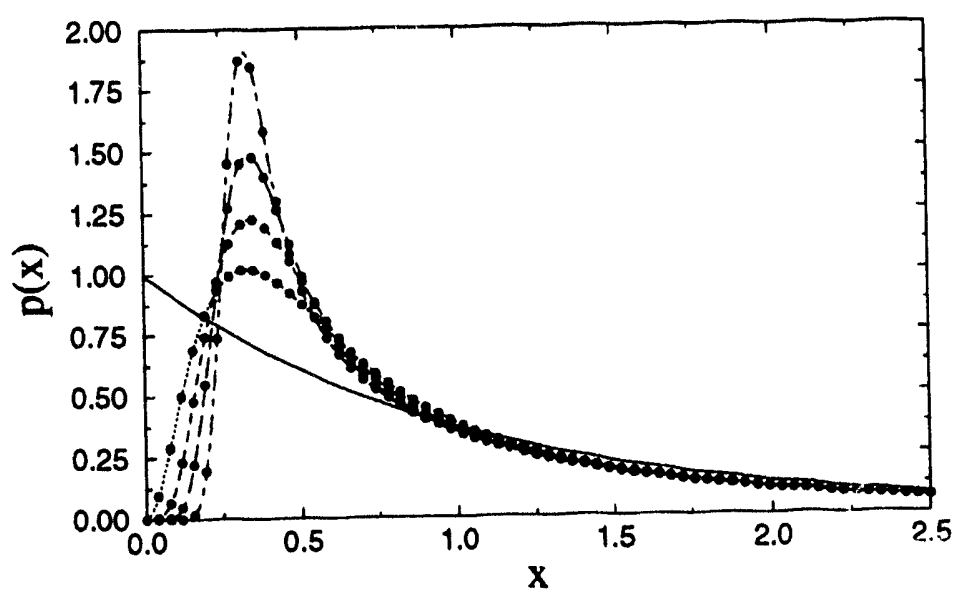

Figure 5.6: The reduced probability distributions [cf. Eq. (5.17)] with $\nu_{\text {eff }}=2$ obtained using the bi-step function model. The solid curve is the $\chi^{2}$-distribution, and the dotted, short, medium and long dashed curves correspond to the smooth step function form with $\left\{\nu_{1}=5, \lambda=.0883\right\},\left\{\nu_{1}=10, \lambda=.0427\right\},\left\{\nu_{1}=20, \lambda=.0210\right\}$ and $\left\{\nu_{1}=40, \lambda=.00833\right\}$, respectively.

\subsection{A Predictive RM/TST Theory}

\subsubsection{The Eigenvalues of the Decay Rate Matrix}

In order to convert the distribution described in the previous section into a predictive formula, one needs to evaluate the eigenvalues of the decay rate matrix $\left\{\gamma_{j}\right\}$ up to an overall multiplicative factor. In the deep tunneling regime, the classical S-Matrix ${ }^{154}$ result is

$$
\gamma_{j}(E)=\frac{1}{2 \pi}\left(\frac{\partial E}{\partial n_{F}}\right) \mathrm{e}^{-2 \theta_{n^{\ddagger}}(E)},
$$

where $j$ is a collective index in correspondence to the $(F-1)$ perpendicular quantum numbers, $\stackrel{n}{\perp}^{\perp}$, which adiabatically connect to the states of the activated complex labeled by $\underline{n}^{\ddagger}, n_{F}$ is the vibrational quantum number associated with the reaction path, and $\theta_{n^{4}}(E)$ is the barrier penetration integral. ${ }^{110}$ This result has the form of Fermi's Golden Rule, ${ }^{155}$

$$
\gamma_{j}(E)=\frac{N_{n^{\ddagger}}}{2 \pi \rho(E)},
$$


where the square of the probability amplitude from a state of the activated complex to the continuum, $N_{n^{\prime}}$, is identified by the semiclassical transmission probability in the deep tunneling regime, $\mathrm{e}^{-2 \theta}$. At higher energies, Carrington et al. ${ }^{156}$ extended this result to approximately include the coupling between the reaction coordinate and the perpendicular modes in the reaction path Hamiltonian. ${ }^{132}$ Within the Feshbach formalism described in Sec. 5.2 and to second order in the coupling to the continuum, they obtain the decay rate matrix:

$$
\Gamma_{\underline{n}, \underline{n}^{\prime}}(E)=\delta_{\underline{n}, \underline{n}^{\prime}} \Gamma_{\underline{n}}(E)+\sum_{\underline{n}^{\ddagger}} 2 \pi \rho\left\langle\underline{n}|H| \underline{n}^{\ddagger}, E\right\rangle\left\langle\underline{n}^{\ddagger}, E|H| \underline{n}_{\underline{n}}^{\prime}\right\rangle,
$$

where $\underset{\sim}{n}, n^{\prime}$ label the zeroth order quasi-bound states, and

$$
\Gamma_{\underline{n}}(E)=\frac{1}{2 \pi}\left(\frac{\partial E}{\partial n_{F}}\right) \ln \left(1+\mathrm{e}^{-2 \theta_{\underline{n} \perp}(E)}\right) .
$$

The first term in Eq. (5.53) is the tunneling contribution obtained within the vibrational adiabatic approximation in the S-Matrix theory; the second term effects the energy transfer between the zeroth order states. In order to obtain the $\gamma_{j}$ 's needed for the distributions of the previous section, one is forced to diagonalize this matrix.

However, in the limit in which there exist good local action-angle variables in the transition state region - i.e. the regime in which the SCTST results of the previous chapters obtain - the eigenvalues of the decay rate matrix are approximately given by

$$
\gamma_{j}=\epsilon\left(1+\mathrm{e}^{2 \theta_{n^{t}}(E)}\right)^{-1} \equiv \epsilon N_{n^{t}},
$$

where $\theta_{n^{1}}(E)$ is now the corresponding good action variable of the reaction coordinate, the quantum numbers $n^{\ddagger}$ are the good local - vibrational or rotational - actions of the activated complex, and $\epsilon$ is a proportionality constant that depends only on energy. The disadvantage of this description relative to that of Carrington et al. ${ }^{156}$ is that here there is no explicit reference to the dynamics of the quasi-bound region and consequently one needs a further computation in order to obtain $\epsilon$. But in calculating the RM/TST reduced probability distributions, Eq. (5.17), knowledge of $\epsilon$ is not required since the formula involves the $\gamma$ 's only up to a proportionality constant. In the next subsection, the RM/TST formalism is used to relate $\epsilon$ to the RM/TST 
parameter, $f$, and thereby make further connection to the result of Carrington et al. $^{156}$

Thus given an $a b$ initio calculation of various "spectroscopic" parameters at the saddle point of the potential energy surface, one can readily insert the transmission probabilities [Eq. (5.55)] into Eq. (5.17) in order to predict the reduced decay rate probability distribution. (The "spectroscopic" parameters include: the frequencies and anharmonicities oltained from the quadratic, cubic and a limited set of the quartic force constants; the rotational constants; and the $\alpha$ 's in the coriolis interaction.)

In summary, therefore, the normalized eigenvalues of $\underset{\tilde{z}}{\Gamma}$ are given by

$$
\gamma_{\underline{n}} / \operatorname{tr}(\underset{\tilde{z}}{\Gamma})=N_{\underline{n}} / N
$$

where $N\left(\equiv \sum_{n} N_{n}\right)$ is the cumulative reaction probability (CRP) and $n_{\sim}$ is a collective index of all the quantum numbers of the activated complex. With this association, Eq. (5.17) becomes

$$
p(x)=(2 \pi)^{-1} \int_{-\infty}^{\infty} d t \mathrm{e}^{i x t} D(t)^{-1 / 2}
$$

where

$$
D(t)=\prod_{\underline{n}}\left(1+2 i t N_{n} / N\right)
$$

\subsubsection{Relation Between $f$ and $\epsilon$}

The value of the proportionality constant, $\epsilon$, in Eq. (5.55) can be related to the effective number of mixed states, $f$, in RM/TST theory. Recall that the microcanonical rate is simply related to the cumulative reaction probability, $N$, and the density of states, $\rho$, by

$$
\bar{\Gamma}=\frac{N}{2 \pi \rho} .
$$

Eq. (5.25) then gives

$$
f=\frac{\operatorname{tr}(\underset{\tilde{n}}{\Gamma})}{\bar{\Gamma}}=\frac{\sum_{\underline{n}} \gamma_{\underline{n}}}{N / 2 \pi \rho}=2 \pi \rho \epsilon\left(\frac{\sum_{\underline{n}} N_{\underline{n}}}{N}\right)=2 \pi \rho \epsilon .
$$


If the states are not mixed, then $f=1$ and $\epsilon=(2 \pi \rho)^{-1}$ as expected. In this case $\epsilon$ is the average energy spacing. If the states are mixed, then $f$ characterizes the average number of mixed levels and $\epsilon$ is the average energy interval which contains $f$ states.

Calculation of $\operatorname{tr}\left(\prod_{\tilde{\tau}}\right)$ could therefore provide a direct check on the applicability of RM/TST theory with large $f$ being favorable. While this calculation is presently not within reach because the reactant density of states is too high, the relation specified in Eq. (5.59) demonstrates that the notion of a set of strongly mixed states in a given energy interval is consistent with the semi-classical model described in Sec. 5.4; the size of that interval being $\epsilon$.

\subsection{Symmetry Considerations}

\subsubsection{The RM/TST Probability Distributions}

The "good" quantum numbers associated with the transition states discussed in Sec. 5.4 are in general not globally conserved quantum numbers. If they were, the molecular Hamiltonian would be integrable, and thus certainly not chaotic, strongly mixed, etc. Most of the "good" quantum numbers of the transition state e.g., the vibrational quantum numbers $\left\{n_{k} ; k=1, \ldots, F-1\right\}$ - are "good" only in the transition state region and thus only relevant for approximating the eigenvalues of $\underset{\tilde{z}}{\Gamma}$. The total angular momentum $J$ (in field-free space), however, is a globally conserved quantum number; and as a consequence states of different $J$ are noninteracting. In applying any statistical theory one should thus be cognizant of all globally conserved quantum numbers - e.g., total angular momentum (in field-free space), global discrete symmetries (the molecular symmetry group), etc. - and invoke the statistical assumption of strong mixing only within each manifold of states labeled by the globally conserved quantum numbers. How the globally conserved symmetries are included into RM/TST is the subject of the present analysis. The role of symmetry in statistical theories has been treated in other contexts by several authors. ${ }^{45,157-159}$

Let us suppose that $\underset{\sim}{\mu}$ are the globally conserved quantum numbers, and 
that $\nu$ are the quantum numbers conserved only locally in the transition state region. The eigenvalues, $\gamma(\underset{\sim}{\mu \nu})$, of the $\underset{\sim}{\Gamma}$ matrix are labeled by the complete set of quantum numbers arising from the direct product of $\underset{\sim}{\mu}$ and $\underset{\sim}{\nu}$. One then applies the RM/TST theory separately for each set of the conserved quantum numbers $\underset{\sim}{\mu}$ : the distribution of unimolecular decay rates for the $\underset{\sim}{\mu}$-manifold of strongly mixed states is given by [noting Eqs. (5.16) and (5.57)]

$$
\begin{aligned}
P_{\underline{\mu}}(\Gamma) & \equiv \bar{\Gamma}_{\underline{\mu}}^{-1} p_{\underline{\mu}}\left(\Gamma / \bar{\Gamma}_{\underline{\mu}}\right) \\
& =\left(2 \pi \bar{\Gamma}_{\underline{\mu}}\right)^{-1} \int_{-\infty}^{\infty} d t \mathrm{e}^{i t \Gamma / \bar{\Gamma}_{\underline{\mu}}} D_{\underline{\mu}}(t)^{-1 / 2},
\end{aligned}
$$

with

$$
D_{\underline{\mu}}(t)=\prod_{\underline{\nu}}\left(1+2 i t N(\underset{\sim}{\mu \nu}) / N_{\underline{\mu}}\right),
$$

where $\bar{\Gamma}_{\underline{\mu}}$ is the average decay rate for the states in the $\underset{\sim}{\mu}$-manifold, $N(\underset{\sim}{\mu \nu})$ is the transmission probability through the state $\mu_{\sim} \underset{\sim}{\nu}$ of the activated complex, and $N_{\mu}[\equiv$ $\sum_{\nu} N(\underset{\sim}{\mu \nu})$ ] is the cumulative reaction probability for the $\underset{\sim}{\mu}$-manifold. [Note that $N(\underset{\sim}{\mu \nu})$ can be distinguished from $N_{\mu}$ because the latter does not have enough quantum numbers to specify a state of the activated complex.] The combined or total distribution is the sum over all the $\underset{\sim}{\mu}$-distributions weighted by the density of states, i.e.,

$$
P_{\text {tot }}(\Gamma)=\sum_{\underline{\mu}} f_{\underline{\mu}} P_{\underline{\mu}}(\Gamma)
$$

with

$$
f_{\underline{\mu}} \equiv \frac{\rho_{\underline{\mu}}}{\rho}
$$

where $\rho_{\underline{\mu}}$ is the density of states of the $\underset{\sim}{\mu}$-manifold and $\rho$ is the total density of states. This is rewritten in terms of the reduced distributions, Eq. (5.17), as

$$
p_{t o t}(x)=\sum_{\underline{\mu}} f_{\underline{\underline{\mu}}} \frac{\bar{\Gamma}}{\bar{\Gamma}_{\underline{\mu}}} p_{\underline{\mu}}\left(x \frac{\bar{\Gamma}}{\Gamma_{\underline{\mu}}}\right) .
$$

Note that this distribution yields the usual transition staie (or RRKM) expression for the average rate:

$$
\bar{\Gamma}=\sum_{\underline{\mu}} f_{\underline{\mu}} \bar{\Gamma}_{\underline{\mu}},
$$


with

$$
\bar{\Gamma}_{\underline{\mu}}=N_{\underline{\mu}} /\left(2 \pi \rho_{\underline{\mu}}\right) \text {. }
$$

The total distribution can also be written, in terms of the cumulative reaction probabilities of the different $\underset{\sim}{\mu}$-manifolds, as

$$
p_{t o t}(x)=\sum_{\underline{\mu}} f_{\underline{\mu}} \eta_{\underline{\mu}} p_{\underline{\mu}}\left(\eta_{\underline{\mu}} x\right) \quad \text { and } \quad \eta_{\underline{\mu}} \equiv \frac{f_{\mu} N}{N_{\underline{\mu}}} .
$$

In the limit that there is only a single symmetry manifold (i.e. $n=1$ ), then $f=1$ and $\eta=1$; hence this expression does include the reduced probability distributions obtained in the Secs. 5.2 and 5.5.

As written, it may seem that to evaluate Eq. (5.65), one needs to Fourier

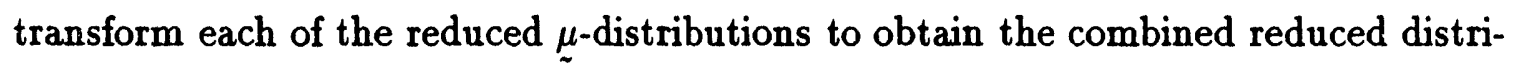
bution. In fact, this is not necessary, After expanding Eq. (5.65) using Eq. (5.17) for each symmetry manifold, transforming the integrating variables, and reversing the oder of integration, one readily obtains:

$$
p(x)=(2 \pi)^{-1} \int_{-\infty}^{\infty} d t \mathrm{e}^{i x t}\left\{\sum_{\underline{\underline{ }}} f_{\underline{\mu}}\left[D_{\underline{\mu}}\left(\frac{t}{\eta_{\underline{\mu}}}\right)\right]^{-1 / 2}\right\}
$$

where

$$
D_{\underline{\mu}}(t)=\prod_{\underline{\nu}}\left(1+2 i t N(\underset{\sim}{\mu \nu}) / N_{\underline{\mu}}\right)
$$

However, in practice it has been found that this expression has more numerical noise than Eq. (5.65), and consequently the latter is used in the applications presented in Chapter 6.

\subsection{2 $\nu_{\text {eff }}$ Using Moments}

The moments of the combined RM/TST reduced distribution can be written analytically in terms of the moments for each manifold as

$$
\left\langle x^{n}\right\rangle=\sum_{\underline{\mu}} f_{\underline{\mu}}\left(\eta_{\underline{\mu}}\right)^{-n}\left\langle x^{n}\right\rangle_{\underline{\mu}}
$$


where $\langle\cdot\rangle_{\underline{\mu}}$ denotes an average with respect to $p_{\underline{\mu}}(x)$. Using the results from Sec. 5.2.4, the effective number of channels for the combined distribution can be written as

$$
\left(\nu_{e f f}^{0}\right)^{-1}=\sum_{\underline{\mu}} f_{\underline{\mu}}\left(\eta_{\underline{\mu}}\right)^{-2}\left(\nu_{\underline{\mu}}^{0}\right)^{-1}+\frac{1}{2} \sum_{\underline{\mu}} f_{\underline{\mu}}\left[\left(\eta_{\underline{\mu}}\right)^{-2}-1\right]
$$

where $\nu_{\underline{\mu}}^{0}$ is the effective number of channels for the $\underset{\sim}{\mu}$-manifold calculated using Eq. (5.28).

\subsection{3 $\nu_{e f f}$ Using the Maximum-Likelihood Method}

The average of the logarithm of the decay rate integrated over the combined $\mathrm{RM} / \mathrm{TST}$ reduced distribution evaluates to:

$$
\begin{aligned}
\langle\ln x\rangle & =\int_{0}^{\infty} d x \ln x \sum_{\underline{\mu}} f_{\underline{\mu}} \eta_{\underline{\mu}} p_{\underline{\mu}}\left(\eta_{\underline{\mu}} x\right) \\
& =\sum_{\underline{\mu}} f_{\underline{\mu}} \int_{0}^{\infty} d y \ln \left(y / \eta_{\underline{\mu}}\right) p_{\underline{\mu}}(y) \\
& =\sum_{\underline{\mu}} f_{\underline{\mu}}\left[(\ln x\rangle_{\underline{\mu}}-\left(\ln \eta_{\underline{\mu}}\right)\right] .
\end{aligned}
$$

Thus, the effective number of channels within the maximum-likelihood formalism is the solution of the transcendental equation,

$$
F\left(\nu_{e f f}^{M L}\right)=\sum_{\underline{\mu}} f_{\underline{\mu}}\left[F\left(\nu_{\underline{\mu}}^{M L}\right)-\left(\ln \eta_{\underline{\mu}}\right)\right]
$$

where $\nu_{\underline{\mu}}^{M L}$ is the effective number of channels for the $\underset{\sim}{\mu}$-manifold calculated using Eq. (5.31), and $F(z)$ is defined in Eq. (5.29).

Similarly the average of the square of the logarithm is:

$$
\left\langle(\ln x)^{2}\right\rangle=\sum_{\underline{\mu}} f_{\underline{\mu}}\left[\left\langle(\ln x)^{2}\right\rangle_{\underline{\underline{\mu}}}-2\left(\ln \eta_{\underline{\mu}}\right)\langle\ln x\rangle_{\underline{\mu}}+\left(\ln \eta_{\underline{\mu}}\right)^{2}\right],
$$

and this can be used to obtain the error estimate in the $\nu_{e f f}^{M L}$ parameter. 


\subsubsection{Summary}

A simple example of the expressions obtained in this section for the RM/TST theory incorporating dynamical symmetries results when the only underlying symmetry divides the states into two uncoupled manifolds, each with an equal density of states. This does not necessarily imply that the corresponding CRP's are equal as the states in a given manifold access only the transition states labeled by the corresponding global symmetry of the manifold. (This case is physically relevant as it can arise if the molecular symmetry group is $C_{s}$.) The combined reduced probability distribution, Eq. (5.65), for this case is

$$
p_{t o t}(x)=\frac{1}{4}\left(\frac{N}{N_{1}} p_{1}\left(\frac{x N}{2 N_{1}}\right)+\frac{N}{N_{2}} p_{2}\left(\frac{x N}{2 N_{2}}\right)\right) .
$$

The moments of this distribution in Eq. (5.67) simplify to:

$$
\left\langle x^{n}\right\rangle=2^{n-1}\left(\left(\frac{N_{1}}{N}\right)^{n}\left\langle x^{n}\right\rangle_{1}+\left(\frac{N_{2}}{N}\right)^{n}\left\langle x^{n}\right\rangle_{2}\right),
$$

which for $n=2$ can be used to obtain the effective number of channels,

$$
\frac{2}{\nu_{e f f}^{0}}=\frac{4\left(N_{1}^{2} / \nu_{e f f, 1}^{0}+N_{2}^{2} / \nu_{e f f, 2}^{0}\right)}{\left(N_{1}+N_{2}\right)^{2}}+\frac{\left(N_{1}-N_{2}\right)^{2}}{\left(N_{1}+N_{2}\right)^{2}},
$$

where $\nu_{e f f, i}^{0}$ is the effective number of channels for the $i$-manifold.

In order to predict a decay rate probability distribution for a given system, one first searches for any conserved symmetries or quantum numbers. Eq. (5.60) is used to obtain the distribution for each of the symmetry blocks. These are combined using Eq. (5.65) to obtain the final result. Note that if only the moments are desired then one first uses the $\gamma(\underset{\sim}{\mu} \underset{\sim}{\nu})$ 's to obtain $\left\langle x^{n}\right\rangle_{\underline{\mu}}$ analytically [e.g. Eq. (5.27) for the second moment] and then use of Eq. (5.67) provides the symmetry adapted RM/TST moments directly. This can also provide a useful check on the numerical evaluation of the probability distribution.

\subsection{Concluding Remarks}

It may be helpful to recapitulate briefly the basic assumptions behind the derivation of the RM/TST model - Eq. (5.17) in Sec. 5.2. (a) The imaginary part 
of the effective Hamiltonian matrix, Eq. (5.2), is treated by first order perturbation theory; this is essentially the assumption that the resonances are non-overlapping ( $\Gamma_{k}<\left|E_{k}-E_{k^{\prime}}\right|$ on the average). (b) The real part of the effective Hamiltonian matrix, Eq. (5.2), is assumed to be a "random" matrix, ${ }^{69,61}$ i.e., the quantum states are assumed to be strongly mixed ("chaotic"). (The only result of random matrix theory which is actually used is that the projections of the eigenstates onto an arbitrary basis are Gaussian random numbers.) Assumption (a) is not very severe since under assumption (b) the state-selected decay rates would be experimentally accessible only if the resonances were non-overlapping or weakly overlapping. However, assumption (b) can hold only if the effective number of mixed states, $f$, is large enough that the association specified by Eq. (5.12) will hold; this can only happen in a region with a high density of states. Thus this analysis is applicable in an intermediate energy regime in which there is a balance between a high density of states and resolvable widths.

The primary new development of the RM/TST model is the generalization to include globally conserved quantities (e.g., total angular momentum and discrete molecular symmetries). The use of the semiclassical transmission probabilities described in Sec. 5.4 also converts this model into a predictive theory. The probability distribution of unimolecular decay rates that results from this RM/TST theory can depend sensitively on which degrees of freedom one assumes are strongly mixed and which are approximately conserved. In practice, therefore, one carries out the statistical calculation for various such assumptions and compares these to the experimental results in order to deduce information about the unimolecular dynamics of the system. 


\section{Chapter 6}

\section{Random Matrix Transition State Theory - Application to Formaldehyde}

\subsection{Introduction}

In this chapter, random matrix / transition state theory (RM/TST) is applied to the $\mathrm{D}_{2} \mathrm{CO}$ dissociation (5.1). The primary new results are the dependence of the probability distributions on total angular momentum, $J$, and the exploration of the dynamical symmetries of the dissociation. Since the results given by the RM/TST theory depend sensitively on which degrees of freedom one assumes are strongly mixed and which are approximately conserved, the calculations were carried out with various assumptions to see which gave the best agreement with experiment. Specifically, $\mathrm{RM} / \mathrm{TST}$ theory is used to obtain the probability distribution for the individual $J$ states of $\mathrm{D}_{2} \mathrm{CO}$ assuming conservation of total angular momentum, $J$, conservation of the angular momentum projection onto a space-fixed axis, $M$, and either: (a) no additional conserved quantities, (b) conservation of $C_{s}$ symmetry, or (c) conservation of $C_{s}$ symmetry and the absolute value of the angular momentum projection onto a body-fixed axis, $|K|$. In addition, the distribution for all the decay rates is obtained under these conditions. Comparison to the experimental distributions indicates that 
case (a) can account for the unimolecular reaction dynamics at high electric field. However, it is possible that $J$-mixing can also provide similar agreement.

In order to apply the RM/TST theory developed in the previous section to $\mathrm{D}_{2} \mathrm{CO}$, the quartic potential and rovibrational consiants must be known at the transition state. However, as Schneider and Thiel ${ }^{160}$ observed for bound state systems, the only quartic derivatives needed to obtain the $x_{i j}$ 's are those of the form $f_{k k l l}$. Handy and coworkers ${ }^{105,106}$ have shown that all of the cubic derivatives and this limited set of quartic derivatives can be efficiently calculated by central differences of analytic second derivatives obtained at second order Møller-Plesset theory (MP2). ${ }^{161}$ This method is directly applicable to the determination of the perturbed Hamiltonian near the transition state and results for the $(J=0)-\mathrm{D}_{2} \mathrm{CO}$ transmission probabilities as illustrated in Figs. 2.5 and 2.6 indicate that there is a small but measurable effect due to the anharmonicity. ${ }^{103}$

The coefficients needed for the rovibrational Hamiltonian are listed in Table 6.1. ${ }^{162}$ Since the transition state geometry of $\mathrm{D}_{2} \mathrm{CO}$ is a near prolate symmetric top - c.f., the asymmetry parameter

$$
\kappa \equiv \frac{2 B-A-C}{A-C}=-.95,
$$

- its rotational energy levels were approximated as a symmetric top. (The rotational constants used for the $\perp$-direction are taken to be the average of the $x$ and $y$ directions.) The vibrational modes, however, do not have a near-degenerate pair, and so may be treated with the non-degenerate perturbative Hamiltonian of Sec. 2.3.2.

\subsection{The Reduced Probability Distributions}

Before comparing to experiment, it is useful to study the degree to which the RM/TST distributions depend on energy and dynamical symmetries. As pointed out in Sec. 5.5, a given system can consist of non-interacting manifolds of states which will not be strongly mixed due to symmetry. For formaldehyde in a Stark field the only rigorously conserved quantum number is $M$, the projection of total angular momentum onto the constant electric field (space fixed) direction. In field-free space, 


\begin{tabular}{|c|c|c|c|c|c|c|c|c|c|c|}
\hline \multirow[b]{2}{*}{$\mathbf{k}$} & \multirow[b]{2}{*}{$\omega_{k}$} & \multicolumn{6}{|c|}{$x_{k, k^{\prime}}$} & \multirow[b]{2}{*}{$\alpha_{k}^{x}$} & \multirow[b]{2}{*}{$\alpha_{k}^{y}$} & \multirow[b]{2}{*}{$\alpha_{k}^{z}$} \\
\hline & & $k^{\prime}=1$ & $k^{\prime}=2$ & $k^{\prime}=3$ & $k^{\prime}=4$ & $k^{\prime}=5$ & $k^{\prime}=6$ & & & \\
\hline $1\left(a^{\prime}\right)$ & 2478 & -15.1 & & & & & & -.001 & -.002 & -.004 \\
\hline $2\left(a^{\prime}\right)$ & 1730 & -19.6 & -7.6 & & & & & -.002 & -.002 & -.011 \\
\hline $3\left(a^{\prime}\right)$ & 1125 & 1.1 & -16.0 & -13.8 & & & & -.003 & -.0002 & -.162 \\
\hline $4\left(a^{\prime \prime}\right)$ & 698 & -14.9 & 2.0 & -4.2 & -3.1 & & & .000 & -.0003 & -.624 \\
\hline $5\left(a^{\prime}\right)$ & 660 & 0.9 & -16.8 & -1.0 & 2.0 & -2.0 & & .003 & -.002 & .529 \\
\hline $6\left(a^{\prime}\right)$ & $1579 i$ & $57.1 i$ & $3.3 i$ & $-14.6 i$ & $28.2 i$ & $-3.0 i$ & -6.7 & $.0003 i$ & $.0006 i$ & $.075 i$ \\
\hline
\end{tabular}

Table 6.1: The coefficients of the rotational Hamiltonian expanded about the saddle point of the $\mathrm{D}_{2} \mathrm{CO}$ potential energy surface. ${ }^{103,162}$ The rotational constants are: $B_{x}=.76, B_{y}=.89$ and $B_{z}=5.59$. All values are in units of $\mathrm{cm}^{-1}$. 
to the extent that the mixing of states caused by the Stark field is negligible, the total angular momentum $J$ and the molecular symmetry $C_{s}$ are also globally conserved." Since $D_{2} C O$ is a near symmetric top, there is also the question of whether $K-$ the projection of $J$ onto the body-fixed axis - is better described as strongly mixed or (approximately) conserved globally. But one can at most require the conservation of $|K|$ because the $+|K|$ and $-|K|$ states are needed to obtain the $C_{s}$ symmetry adapted states. (The conditions for labeling a given transition state as even $\left(A^{\prime}\right)$ or odd $\left(A^{\prime \prime}\right)$ are given by Miller. ${ }^{163}$ Note that $\nu_{4}$, the out-of-plane mode, is the only vibrational mode which is non-trivial with respect to $C_{8}$ symmetry.)

Thus four cases of dyramical symmetry which are pertinent to the $D_{2} \mathrm{CO}$ dissociation are:

(a) $J$ and $M$ are the conserved quantities.

(b) $J, M$, and $C_{s}$ are the conserved quantities.

(c) $J, M,|K|$, and $C_{s}$ are the conserved quantities.

(d) $M$ and $C_{s}$ are the conserved quantities, with $J$ partially broken and $|K|$ less so.

Although case (d) is potentially very relevant to the dynamics, we can not use the present form of RM/TST to obtain the corresponding distributions since it would require the use of "partially" mixed states. For the remaining cases, we can construct $J$-resolved distributions, i.e.

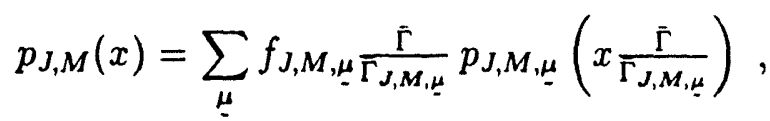

where $p_{J, M, \mu}$ are the RM/TST reduced probability distributions labeled by $J, M$ and $\underset{\sim}{\mu}$, and the sum is over all values of $\mu$ which are accessed in the experiment. (In the symmetric top limit, the transmission probabilities are independent of $M$ and consequently so is the $J$-resolved distribution; $p_{J, M}=p_{J}$.)

" $C_{\delta}$ is the symmetry of the transition state and the related reaction path. There are actually two such (symmetrically equivalent) transition states and they combine to give a molecular symmetry for $\mathrm{D}_{2} \mathrm{CO}$ as $C_{2 V}$. Because these two transition states are far enough apart to be non-interacting, one can use the lower symmetry $C_{s}$ as we do here. ${ }^{159}$ 
In case (a), the sum in Eq. (6.2) collapses into a single term: Eq. (5.60) labeled by $J$ and $M$. In the remaining cases, it is also necessary to make an assumption concerned with which $\mu$-states are accessed by the experiment and consequently should be included in the sum in Eq. (6.2). In case (b), we make the most democratic choice by including all such states. Since states in $A^{\prime}$ or $A^{\prime \prime}$ will have essentially the same density of states, the $J$-resolved distribution in case $(b)$ is obtained using Eq. (6.2) with $f_{i}=\frac{1}{2}$, in analogy to Eq. (5.72). In case (c), if $|K|$ is conserved in the preparation of the metastable states, then only those states with $|K|=J$ will be observed in the experiments. With this restriction, the sum in Eq. (6.2) only includes the two $C$ s states corresponding to $|K|=J$ and is reduced to Eq. (5.72) as in case (b).

In Figs. 6.1-6.3, the RM/TST $J$-resolved reduced probability distributions are presented at selected sample energies relative to the bare barrier and for different values of $J$. (The energies are chosen to sample energy regimes well below, just above and well above the zero point energy adjusted barrier.) The figures indicate that there is a pronounced variation in the shape of the predicted distributions as a function of energy, $J$, and symmetry. In particular, the distributions tend to be narrower and more strongly peaked with either increasing energy or decreasing dynamical symmetry.

As has already been discussed in previous chapters, the effective number of channels, $\nu_{\text {eff }}$, is often used to characterize the probability distributions by a single parameter. However, one should be careful not to rely too heavily on this measure. One reason for this is that very different distributions can have the same value of $\nu_{\text {eff }}$ as illustrated in Sec. 5.3. A second reason is that if Eq. (5.28) is used to obtain a $\nu_{\text {eff }}$ for a finite number of observables - we refer to this value as $\nu_{e f f}^{0}$ - then the largest rates will bias the value of $\nu_{\text {eff }}^{0}$ unequally. Moreover, large rates correspond to broader peaks which are poorly resolved experimentally. This bias is partially corrected by using the maximum-likelihood method ${ }^{63,68}$ in which the average of the logarithm of the rates is used to determine $\nu_{\text {eff }}^{M L}{ }^{\dagger}$

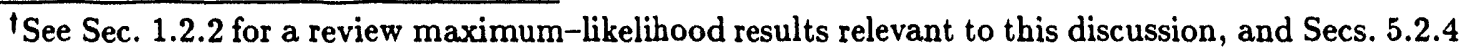
and 5.5.3 for a discussion on the computation of $\nu_{e f f}^{M L}$ for RM/TST distributions.
} 


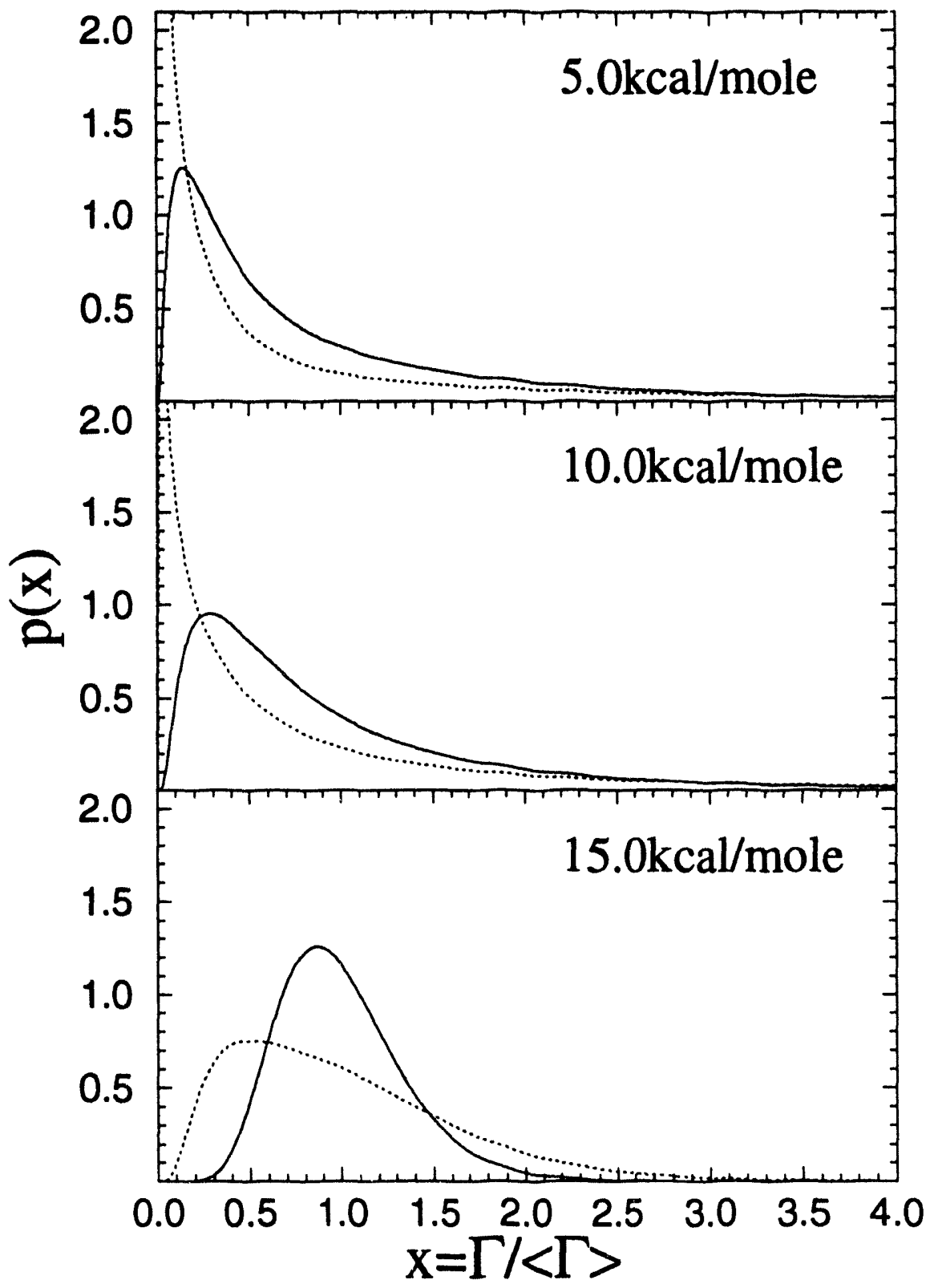

Figure 6.1: RM/TST probability distributions with $J=0$ at various energies. The solid curves are obtained assuming no additional conserved quantities, case $(a)$. The short-dashed curves are obtained assuming that $C_{s}$ symmetry is also obeyed, case (b). 


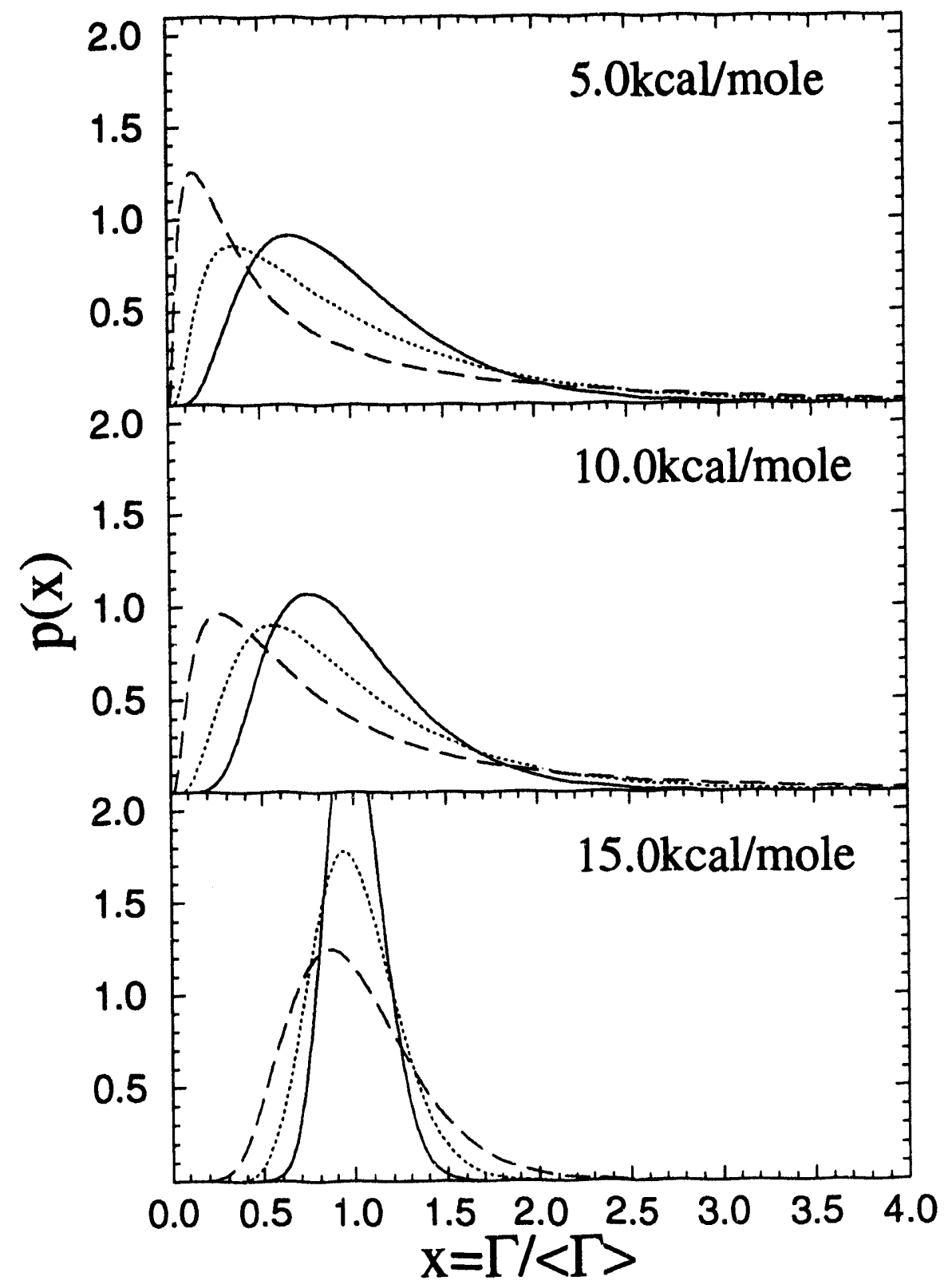

Figure 6.2: RM/TST probability distributions with $J=2$ at various energies. The solid and short-dashed curves correspond to the same cases as in Fig. 6.1. The longdashed curves are obtained assuming that $C_{s}$ symmetry and $|K|$ are also obeyed, case (c). 


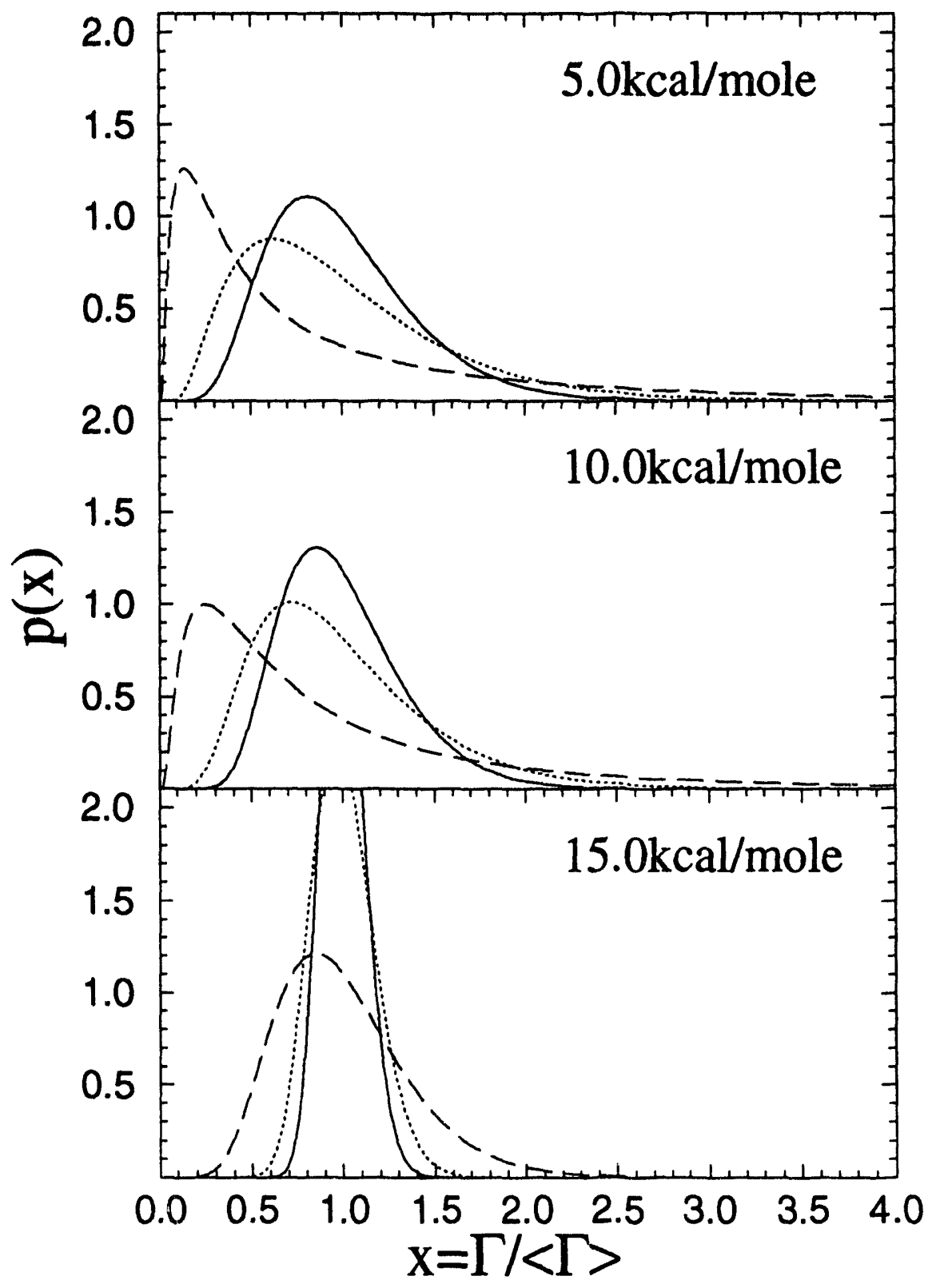

Figure 6.3: RM/TST probability distributions with $J=4$ at various energies. All curves correspond to the same cases as in Figs. 6.1 and 6.2. 


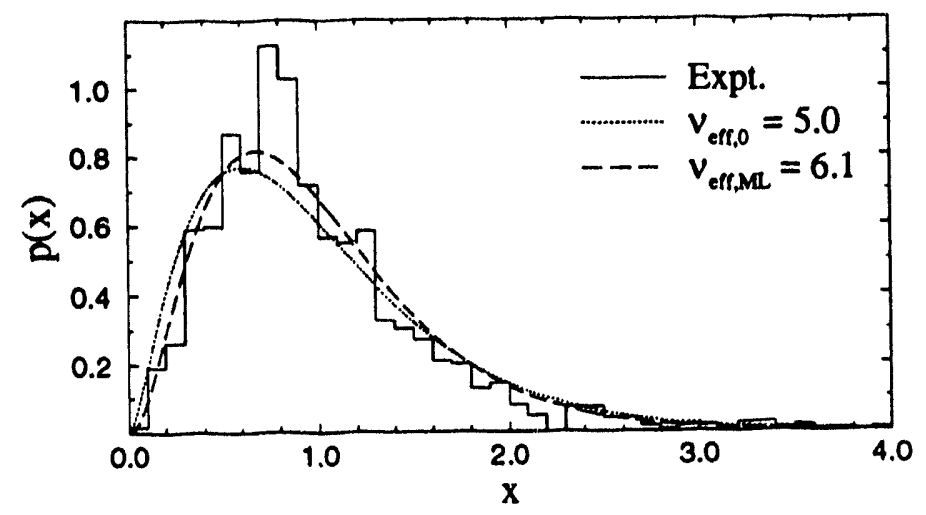

Figure 6.4: $\chi^{2}$-distributions with best fit values of $\nu_{e f f}^{0}$ and $\nu_{e f f}^{M L}$ versus the experimental histogram. ${ }^{99}$

To illustrate the qualitative differences in the best fit values of $\nu_{\text {eff }}$ using either of these methods, the experimental histogram of the $\mathrm{D}_{2} \mathrm{CO}$ decay rates is compared in Fig. 6.4 to the $\chi^{2}$-distributions with $\nu$ determined by the best fit values of $\nu_{e f f}^{0}$ and $\nu_{e f f}^{M L}$. It should be clear that while the difference in the fitted values of $\nu_{e f f}$ is large, the difference between the two $\chi^{2}$-distributions is small. Although both distributions are in reasonable agreement with the experimental result (the histogram), one can discern qualitative differences, e.g., the experimental distribution is narrower and dies off faster at the high end. Thus, the values of $\nu_{\text {eff }}$ determined by either fitting procedure are not presented for the RM/TST distributions illustrated in Figs. 6.1-6.3 as this would not provide further information about how strongly the RM/TST distributions differ from the $\chi^{2}$-distributions. However, in Sec. 6.4 these values are presented both for the experimental distributions and the RM/TST distributions as a (weak) measure of the agreement or disagreement between them.

\section{3 $\mathrm{D}_{2} \mathrm{CO}$ Barrier Height}

In the previous section, the RM/TST distributions were shown to vary strongly with $E$, the energy relative to the bare barrier:

$$
E=h \nu_{\text {exp }}+E_{Z P E}^{0}-V_{0}
$$


where $\nu_{\text {exp }}$ is the frequency of the excitation in the experiment, $E_{Z P E}^{0}$ is the zero point energy (ZPE) of the bound state and $V_{0}$ is the bare barrier height. Since the theoretical calculations are performed about the barrier, the values of $E_{Z P E}^{0}$ and $V_{0}$ are required in order to match to the experiment. $E_{Z P E}^{0}$ is readily computed from the ground state force field of formalde:lyde; the force field has been computed by Handy et al. ${ }^{105,106}$ using analytic second derivatives within MP2 and compares well with the experimental results of Duncan and Mallinson ${ }^{164}$ and Reisner et al. ${ }^{165}$ (For $\mathrm{D}_{2} \mathrm{CO}$, $E_{Z P E}^{0}=13.0 \mathrm{kcal} /$ mole.) However, $a b$ initio calculations are just beginning to reach agreement on $V_{0}^{166}$ and it is useful to obtain an estimate of the barrier height by a fit of the $J$-resolved experimental results with the semiclassical anharmonic transition state model described in Sec. 5.4.

The barrier height can be determined by varying the energy of the RM/TST cumulative reaction probability (CRP) calculation until agreement is reached with the experimentally inferred CRP. The latter quantity can be obtained, in principle, by either of two equivalent methods. The most straightforward is to write:

$$
N=2 \pi \rho \bar{\Gamma} \quad \text { with } \quad \rho=\sum_{J} \rho_{J} .
$$

Alternatively, the CRP is the sum:

$$
N=\sum_{J} N_{J}=2 \pi \sum_{J} \rho_{J} \bar{\Gamma}_{J}
$$

The two methods are clearly equivalent if $\bar{\Gamma}$ is the weighted average of $\bar{\Gamma}_{J}$ as in Eq. (5.64). The extent to which these expressions are not equal is therefore a consistency check on the experimentally ${ }^{21}$ obtained rates and density of states, $\rho_{\text {exp }}(J)$. In general, one also needs to include the appropriate symmetry numbers in the rate expressions. ${ }^{\ddagger}$ This is hidden in Eq. (6.4) and subsequent expressions by including the symmetry numbers in the density of states.

A difficulty in carrying out this procedure arises from the nature of the Stark level-crossing spectroscopy experiment. $S_{0}$ resonances are necessarily observed at varying electric field strengths, giving rise to a density of states anomaly which is

\footnotetext{
'See remarks at bottom of page 113 .
} 
not directly accounted for in the calculation. The density of $S_{0}$ vibrational states is observed to increase by a factor of four over a typical electric field strength scan of $20 \mathrm{kV} / \mathrm{cm} .{ }^{21}$ This increase in density of states is attributed to partial breakdown of the $J$ quantum number. The varying density of states introduces an uncertainty in the determination of the experimental CRP and hence in the calculated decay rate distributions. To limit this effect, the density of states used in the CRP calculation is obtained by extrapolating the observed density of states to zero electric field. ${ }^{21}$ Although all of the data is used to obtain the average rate, a restriction to those rates obtained at low electric field yields at most a $.2 \mathrm{kcal} / \mathrm{mole}$ lowering in the barrier height, suggesting that the electric field has a small effect on the average rate.

Since cases (b) and (c) are the most likely candidates to describe the dynamics of the reaction at zero electric field, only these cases are considered in calculating the barrier height. In case (c), the restriction to include only the $|K|=J$ states lowers the CRP relative to that of case (b), and consequently a lower barrier is needed to fit to the experimental value. Thus a fit to the experimentally inferred CRP also provides some information about the metastable states which are being accessed by the experiment.

If all the $K$ states are accessed in the experiment, as in case (b), then the density of states is given by a state count, i.e.,

$$
\rho(J)=(2 J+1) \rho(0)
$$

where $\rho(J)$ is the density of states as a function of $J$. The density of states is thus determined by a single multiplicative constant; the first two experimental columns in Table 6.2 present the results of this calculation with either $\rho_{\exp }(0)$ or $\rho_{\exp }(1)$ fixing this multiplicative constant. (Note that $\rho_{\exp }(0)$ is a further extrapolation of the observed $\rho_{\text {exp }}(J)$ assuming linear dependence with a slope of (.45 \pm .16$)$ instead of the statistical value of $2 .{ }^{21}$ ) If only the $|K|=J$ states are accessed in the experiment, as in case (c), then the density of states will be independent of $J$. However, the density of states observed experimentally by Polik et al. ${ }^{21}$ did not quite follow either of these statistical regimes. In fact, they found that "although the density of states increases slightly with $J, K$ appears to be predominantly conserved". ${ }^{21}$ The last experimental 


\begin{tabular}{lllrll}
\hline \hline & ${\text { RM} / \text { TST }^{\mathrm{a}}}$ & RM TST $^{\mathrm{b}}$ & Exp. $^{\mathrm{c}}$ & Exp. $^{\mathrm{d}}$ & Exp. $^{\mathrm{e}}$ \\
\hline$N_{J=1}$ & 0.98 & 1.97 & 3.47 & 1.36 & 1.36 \\
$N_{J=2}$ & 1.59 & 1.90 & 4.09 & 1.60 & 1.50 \\
$N_{J=3}$ & 2.13 & 1.78 & 6.31 & 2.46 & 1.72 \\
$N_{J=4}$ & 2.58 & 1.63 & 9.76 & 3.81 & 2.70 \\
$\sum_{J} N_{J}$ & 7.28 & 7.28 & 23.63 & 9.22 & 7.28 \\
$2 \pi \rho \bar{\Gamma}$ & 7.28 & 7.28 & 23.11 & 9.02 & 7.13 \\
\hline
\end{tabular}

${ }^{a}$ For case (b).

${ }^{b}$ For case (c).

${ }^{c} U \operatorname{sing} \rho(J)=(2 J+1) \rho_{\exp }(0)$.

${ }^{d}$ Using $\rho(J)=(2 J+1) \rho_{\text {exp }}(1) / 3$.

${ }^{e}$ Using $\rho(J)=\rho_{\text {exp }}(J)$ in the case that $K$ is predominantly conserved.

Table 6.2: The cumulative reaction probability for the $J$-resolved and combined probability distributions compared to experiment. ${ }^{99}$

column in Table 6.2 presents the CRP's obtained using $\rho_{\text {exp }}(J)$. The relative ratios in the calculation of the total CRP using Eqs. (6.4a) and (6.4b) between each of the experimental columns of Table 6.2 are similar, and thus this consistency check does not resolve the issue of which is the correct choice of the density of states.

The RM/TST CRP's presented in Table 6.2 are obtained at a value of the barrier height such that the total CRP agrees with the experimentally inferred CRP using $\rho_{\text {exp }}(J)$. In case $(b)$, the CRP is fit to the third experimental column with $V_{0}=85.4 \pm 1.0 \mathrm{kcal} / \mathrm{mole} ; \mathrm{a}$ decrease in the barrier height by $.2 \mathrm{kcal} / \mathrm{mole}$ would increase the total CRP to match that of the second experimental column whereas a $1.3 \mathrm{kcal} / \mathrm{mole}$ decrease is required to match the first. In case (c), the CRP is fit to the third experimental column with $V_{0}=84.1 \pm 1.0 \mathrm{kcal} / \mathrm{mole}$; a decrease in the barrier height by $.3 \mathrm{kcal} / \mathrm{mole}$ would increase the total CRP to match that of the second experimental column whereas a $2.3 \mathrm{kcal} / \mathrm{mole}$ decrease is required to match the first. In both sets of cases, the error arises from the same sources as in Ref. 21 with an additional $.2 \mathrm{kcal} / \mathrm{mole}$ arising from the energy spread of the observed rates. Assuming that the dependence of the density of states on $J$ is correct in the experiments, then the barrier heights are taken to be those which matched the 


\begin{tabular}{llc}
\hline \hline Method & Barrier Height & Reference \\
\hline MCSCF+CI & $86.0 \pm 2.5$ & 167 \\
MP4SDTQ & 85.9 & 168 \\
CCSDT-1 & 86.8 & 166 \\
RRKM $(J=0)$ & $84.6 \pm 0.8$ & 21 \\
RM/TST $(b)$ & $85.4 \pm 1.0$ & - \\
RM/TST $(c)$ & $84.1 \pm 1.0$ & - \\
\hline \hline
\end{tabular}

Table 6.3: $A b$ initio and empirical bare barrier heights in units of $\mathrm{kcal} / \mathrm{mole}$ for the $\mathrm{D}_{2} \mathrm{CO} \rightarrow \mathrm{D}_{2}+\mathrm{CO}$ reaction.

third experimental column. In any event, we note that a change in the RM/TST distributions caused by up to a $.5 \mathrm{kcal} /$ mole decrease of the barrier height does not significantly alter the qualitative features of the RM/TST distributions.

In Table 6.3, the RM/TST results for the bare barrier are summarized and compared to previous $a b$ initio results and to the earlier result of Polik et $a .^{21} \mathrm{ob}$ tained using a harmonic RRKM fit of all of the rates extrapolated to $J=0$. The RM/TST barrier heights are within the error bars of the $J=0$ extrapolation. Although the result under case $(b)$ is closer to the $a b$ initio results than that for case (c), the agreement is insufficient to conclude that all the $K$ states are accessed in the experiment.

\subsection{Comparison to Experiment}

A comparison between the RM/TST and experimental reduced probability distributions should reveal which local quantum numbers are approximately conserved throughout the reaction, and which are strongly mixed. In case (b), for example, the energy of the experiment relative to the bare barrier is $8.7 \mathrm{kcal} / \mathrm{mole}$. Since this is near the zero point energy of the transition state $(\sim 9.5 \mathrm{kcal} / \mathrm{mole})$, many of the contributing states of the activated complex are in the tunneling regime and an adequate description of their transmission probabilities must account for tunneling and anharmonicity. This, therefore, justifies the need for the semiclassical transition 
state theory described in Sec. 5.4.

\subsubsection{The $J$-Resolved Distributions}

The $J$-resolved RM/TST distributions are compared to the experimental histograms for individual $J$ states $^{21,99}$ in Fig. 6.5. (Note that in case (a), the calculations are performed using the barrier height obtained in case (b).) The poor agreement between the histograms and distributions obtained for case (c) indicates that $|K|$ is not conserved. The experimental distribution seems to lie in between the theoretical distributions for cases $(a)$ and (b), and this suggests that the system has some mixing between the $C_{s}$ states, but not complete mixing.

For completeness, the values of $\nu_{\text {eff }}$ for cases (a), (b) and (c) are compared to those of the experiments in Table 6.4. As is the case for the total distribution described in Sec. 6.2 there is disagreement between the experimentally determined values, $\nu_{\text {eff }}^{0}$ and $\nu_{\text {eff }}^{M L}$, providing further support for their limited utility. The difference in the values of $\nu_{e f f}^{0}$ and $\nu_{e f f}^{M L}$ for the RM/TST distributions reflects the fact that these distributions are not $\chi^{2}$ and consequently fits based on different observables will differ. Similarly the large errors in the maximum-likelihood $\nu_{\text {eff }}$ 's for the $\mathrm{RM} / \mathrm{TST}$ distributions are a consequence of the fact that the logarithm constraint in the maximum likelihood method is not the correct constraint for the RM/TST distributions.

Nonetheless the experimentally obtained values of $\nu_{\text {eff }}$ fall roughly in between the RM/TST results for cases (a) and (b). It should also be noted that to a good approximation the primary effect of $C_{s}$ symmetry is to halve the available states of the activated complex (i.e. $\nu_{e f f, a} \approx 2 \nu_{e f f, b}$ ) and this is seen in the RM/TST results in Table 6.4. Thus, the $J$-resolved results indicate that the $K$ states are strongly mixed and that $C_{s}$ symmetry is to some extent broken, but not completely mixed in the random matrix sense. 


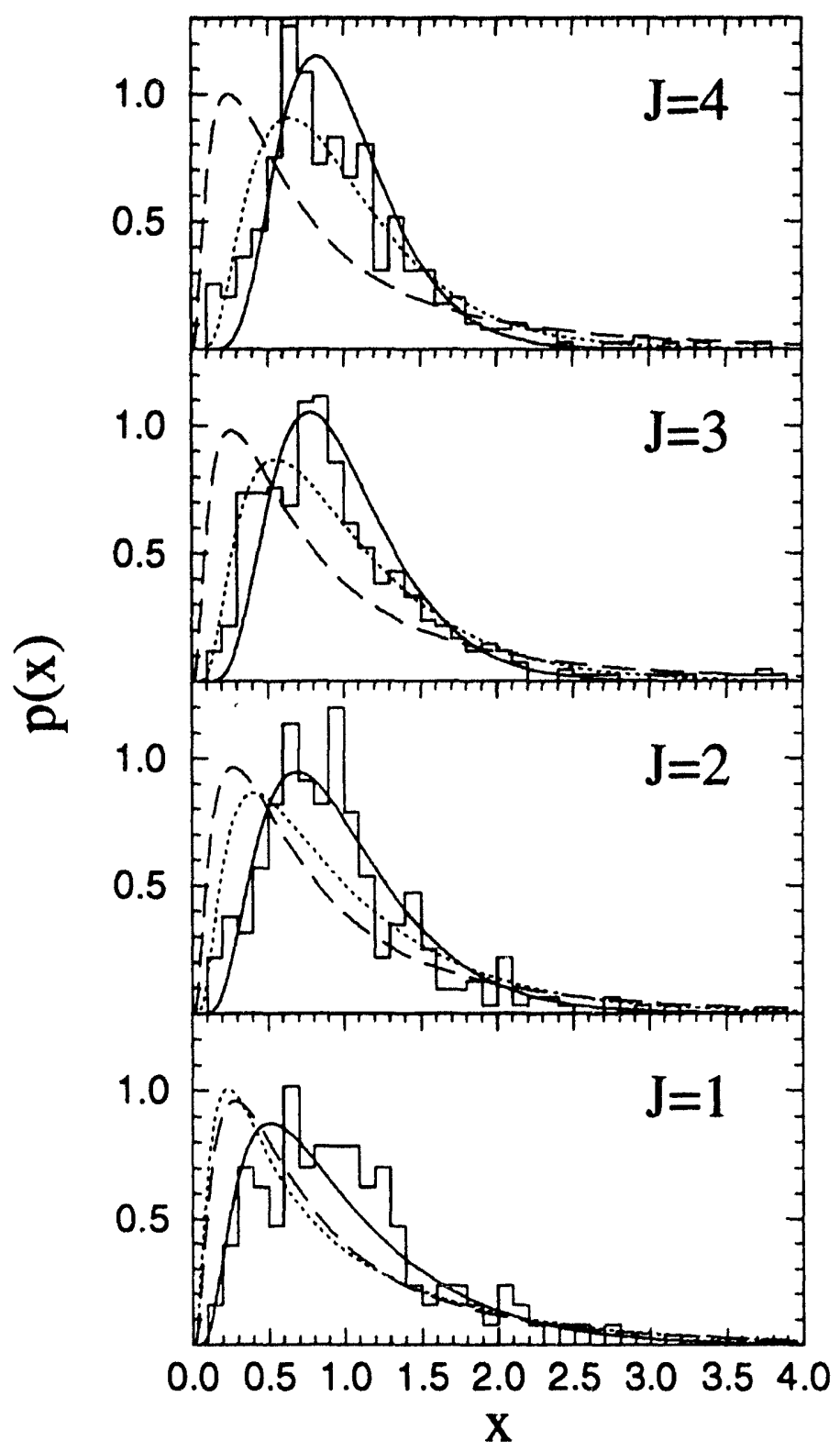

Figure 6.5: RM/TST probability distributions at the energy of the experiment for various values of $J$ compared to the $J$-resolved experimental histograms. ${ }^{21,99}$ The solid, short-dashed and long-dashed curves are as in Figs. 6.1 and 6.2. 


\begin{tabular}{|c|c|c|c|c|c|c|}
\hline \multirow[b]{3}{*}{$\mathrm{J}$} & \multicolumn{6}{|c|}{ Experimental $\nu_{\text {eff }}$} \\
\hline & \multicolumn{2}{|c|}{ All Rates } & \multicolumn{2}{|c|}{ Low E-Field } & \multicolumn{2}{|c|}{ High E-Field } \\
\hline & $\nu_{e f f}^{0}$ & $\nu_{e f f}^{M L}$ & $\nu_{e f f}^{0}$ & $\nu_{e f f}^{M L}$ & $\nu_{\text {eff }}^{0}$ & $\nu_{\text {eff }}^{M L}$ \\
\hline 1 & 7.0 & $6.8 \pm 1.0$ & 5.2 & $4.5 \pm 1.6$ & 7.4 & $7.8 \pm 1.4$ \\
\hline 2 & 4.0 & $6.0 \pm 1.5$ & 2.0 & $3.6 \pm 1.0$ & 8.0 & $8.4 \pm 1.7$ \\
\hline 3 & 4.7 & $6.3 \pm 0.9$ & 3.3 & $4.2 \pm 1.5$ & 9.2 & $9.0 \pm 1.4$ \\
\hline 4 & 6.2 & $6.9 \pm 1.1$ & 5.3 & $6.0 \pm 1.4$ & 7.7 & $7.6 \pm 1.2$ \\
\hline all & 5.0 & $6.1 \pm 0.7$ & 3.4 & $4.3 \pm 0.9$ & 7.4 & $7.7 \pm 0.8$ \\
\hline
\end{tabular}

\begin{tabular}{|c|c|c|c|c|c|c|}
\hline \multirow[b]{3}{*}{$\mathrm{J}$} & \multicolumn{6}{|c|}{ RM/TST $\nu_{\text {eff }}$} \\
\hline & \multicolumn{2}{|c|}{ case $(a)$} & \multicolumn{2}{|c|}{ case (b) } & \multicolumn{2}{|c|}{ case (c) } \\
\hline & $\nu_{e f f, a}^{0}$ & $\nu_{e f f, a}^{M L}$ & $\nu_{e f f, b}^{0}$ & $\nu_{e f f, b}^{M L}$ & $\nu_{e f f, c}^{0}$ & $\nu_{e f f, c}^{M L}$ \\
\hline 1 & 4.61 & $5.22 \pm 200$ & 2.12 & $2.68 \pm 4.4$ & 2.08 & $2.84 \pm 6.2$ \\
\hline 2 & 7.66 & $8.22 \pm 38$ & 3.63 & $4.21 \pm 45$ & 2.04 & $2.80 \pm 5.8$ \\
\hline 3 & 10.7 & $11.2 \pm 2.0$ & 5.11 & $5.70 \pm 200$ & 1.97 & $2.72 \pm 5.3$ \\
\hline 4 & 13.6 & $14.0 \pm 4.9$ & 6.55 & $7.14 \pm 110$ & 1.89 & $2.63 \pm 4.7$ \\
\hline all $^{a}$ & 8.70 & $9.62 \pm 5.7$ & 4.14 & $4.94 \pm 6.7$ & 1.35 & $2.22 \pm 2.3$ \\
\hline all $^{b}$ & 8.70 & $8.78 \pm 8.1$ & 4.25 & $4.60 \pm 2.1$ & 1.63 & $2.44 \pm 3.0$ \\
\hline
\end{tabular}

${ }^{a}$ Obtained using $(2 J+1)$ weights.

bobtained using $\rho_{\exp }(J)$ for which $K$ is predominantly conserved.

Table 6.4: The values of $\nu_{\text {eff }}$ for the $J$-resolved and combined probability distributions obtained from the experimental results of Polik et al. ${ }^{21}$ (top panel) and from the RM/TST results (bottom panel). Recall that $\nu_{\text {eff }}^{0}$ is computed from the second moment, Eq. (5.28), and $\nu_{e f f}^{M L}$ is computed using the maximum-likelihood method. In the RM/TST results, $\nu_{e f f, a}^{*}, \nu_{e f f, b}^{*}$ and $\nu_{e f f, c}^{*}$ are obtained under cases $(a),(b)$, and (c), respectively. 


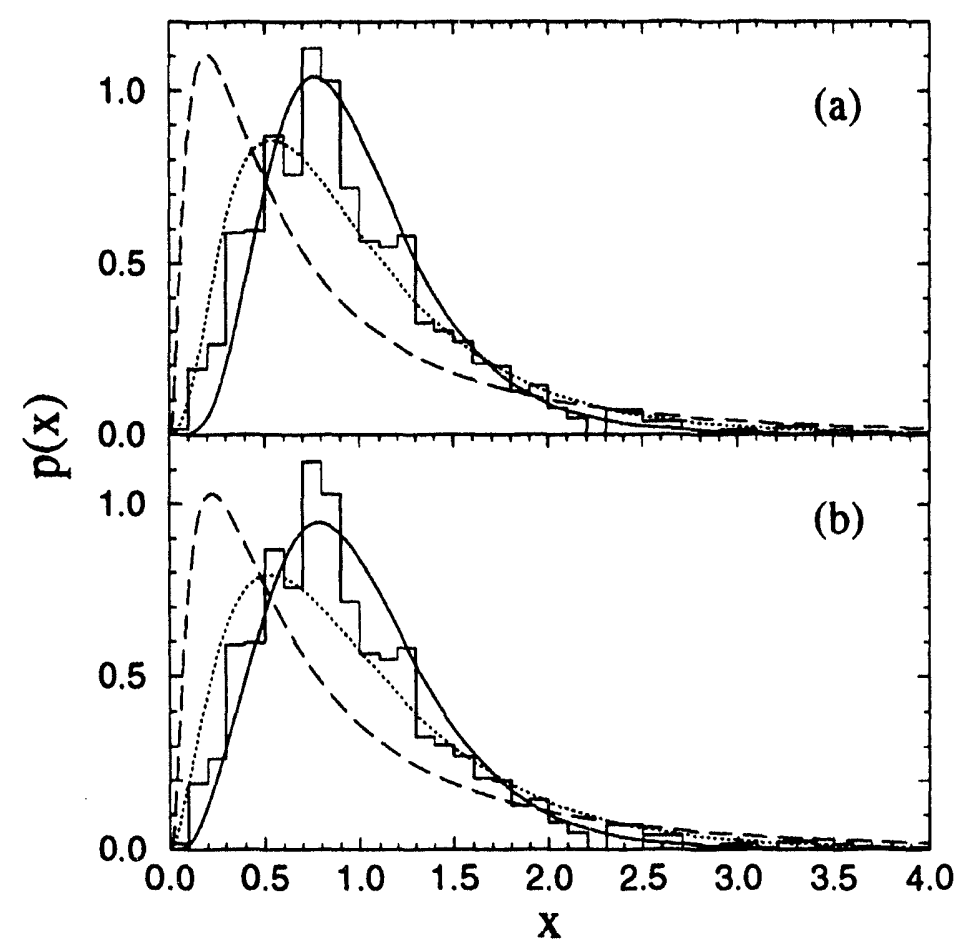

Figure 6.6: RM/TST probability distributions at the energy of the experiment with $\rho(J)=(2 J+1) \rho_{\text {exp }}(0)$ in the top panel (a) and with $\rho(J)=\rho_{\text {exp }}(J)$ in the bottom panel (b) compared to the experimental histogram for all rates. The solid, shortdashed and the long-dashed curves are obtained assuming cases (a), (b) and (c), respectively.

\subsubsection{The Combined Distributions}

In order to combine the $J$-resolved RM/TST distributions using Eq. (5.65), the relative density of states of each $J$ manifold must be determined. The combined RM/TST distributions using either the statistical density of states, where $\rho(J)=$ $(2 J+1) \rho(0)$, or the experimental densities of states, $\rho_{\exp }(J)$, are compared to the experimental histogram in Fig. 6.6. As is the case for the $J$-resolved distributions, the total RM/TST distributions for cases (a) and (b) bracket the observed histogram while the peak of the distribution for case $(c)$ is shifted somewhat to the left of the experimental histogram. This once again leads to the possible conclusion that states of different $C_{s}$ symmetry are partially mixed by the Stark field. 
While the above calculations suggest total breakdown of the $|K|$ quantum number as a candidate for the origin of the additional degrees of freedom observed in the experimental decay rate distributions, it is acknowledged that several other potential sources for degrees of freedom also exist. First, vibrational anharmonicity arising from still higher order terms in the transition state region of the potential energy surface could result in a higher $\nu_{\text {eff }} .{ }^{100}$ Second, $J$ breakdown induced by the Stark field will increase the number of available decay channels and could provide similar agreement between experimental and calculated distributions. Third, only the limiting cases of no breakdown or complete breakdown of the $|K|$ quantum number have been considered in this treatment. An intermediate case - e.g. case (d) of partial $K$ breakdown, in combination with other decay channels, could account for the experimental distributions. Note that this would increase the barrier height - although by less than $1.0 \mathrm{kcal} / \mathrm{mole}$ - because the sum in the CRP would now include additional states of the activated complex. While the results of this section have been inferred through the examination of the decay rate distributions observed in $S_{0} \mathrm{D}_{2} \mathrm{CO}$, other experimental data suggest the opposite conclusion, namely that $|K|$ may not be strongly mixed. The lack of a $2 J+1$ dependency of the experimental density of states and the lack of a $1 /(2 J+1)$ dependency of the average squared $S_{1}-S_{0}$ coupling matrix element each suggest that $|K|$ is only partially mixed. ${ }^{21}$

\subsubsection{The Effect of The Electric Field}

Thus far the effect of the electric field on the rates has been ignored since the energetic effect is minimal. However, the isotropy of space is broken in the presence of an electric field and this minimally breaks the $C_{s}$ symmetry in the moleculefixed frame of the transition state at sufficiently high field strengths. The loss of $C_{s}$ symmetry can have a strong effect on the statistical distributions as evidenced by the difference in the RM/TST distributions in Fig. 6.6. In fact, the results for cases (a) and (b) might suggest that at low electric field strengths $C_{s}$ symmetry would be conserved, whereas at high electric field strengths it would be broken. This is further suggested, for example, in Figure 10 of Ref. 99, in which $\nu_{\text {eff }}$ is plotted versus the 


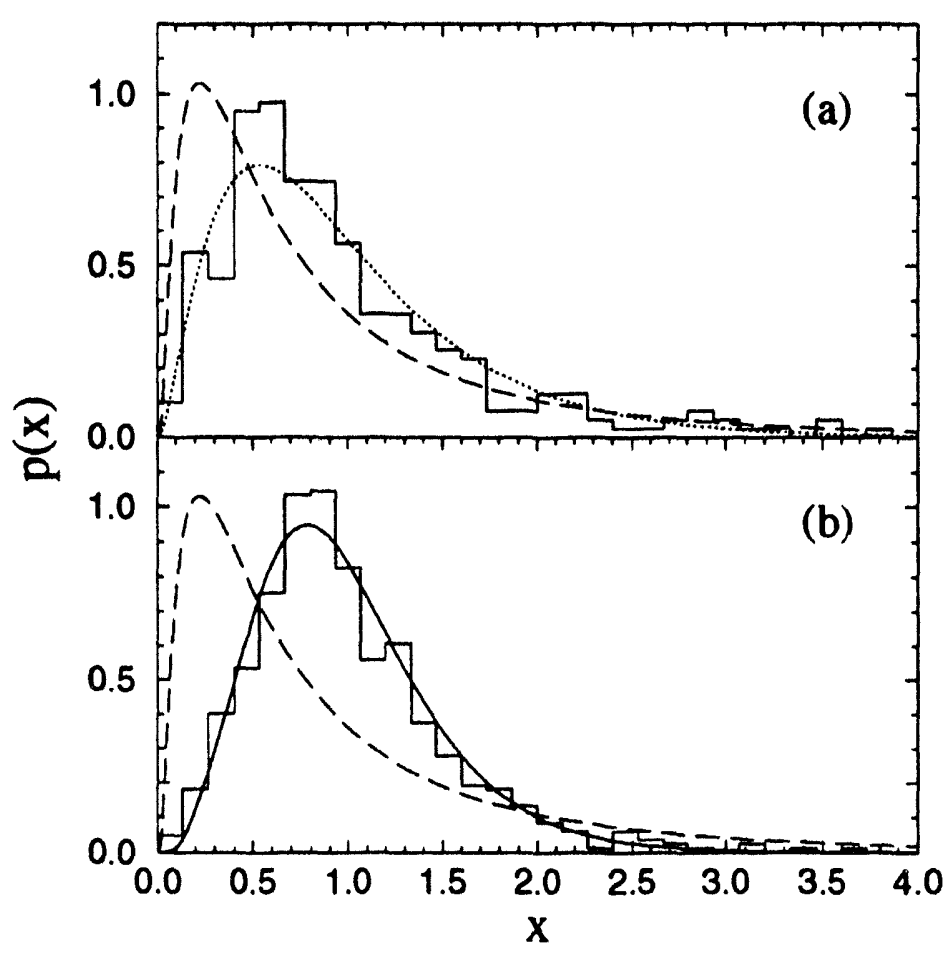

Figure 6.7: RM/TST probability distributions at the energy of the experiment compared to the experimental histogram for rates at low electric field (a) and at high electric field (b). The RM/TST distributions are obtained with $\rho(J)=\rho_{\exp }(J)$, and the solid, short-dashed and long-dashed curves are obtained as in Fig. 6.6.

electric field; although a linear fit is shown in that presentation, its behavior is more like that of a sigmoid with $\nu_{\text {eff }}$ going from $\sim 4$ in the low field region to $\sim 8$ in the high field region. The ratio of two in $\nu_{\text {eff }}$ between these two regions indicates that the electric field is breaking a symmetry which has only two labels, such as $C_{8}$ symmetry.

In Fig. 6.7a, the experimental histogram is obtained using all of the 212 observed rates with electric field strength less than $6.5 \mathrm{kV} / \mathrm{cm}$ and is compared to the $\mathrm{RM} / \mathrm{TST}$ distributions with $C_{s}$ symmetry. In Fig. $6.7 b$, the experimental histogram is obtained using all of the 617 observed rates with electric field strength greater than $12.0 \mathrm{kV} / \mathrm{cm}$ and is compared to the RM/TST distributions without $C_{s}$ symmetry. For completeness, the histograms labeled by $J$ for each of these electric field cases are also compared to the $J$-resolved distributions in Fig. 6.8. Although there are 


\section{Low E-Field}

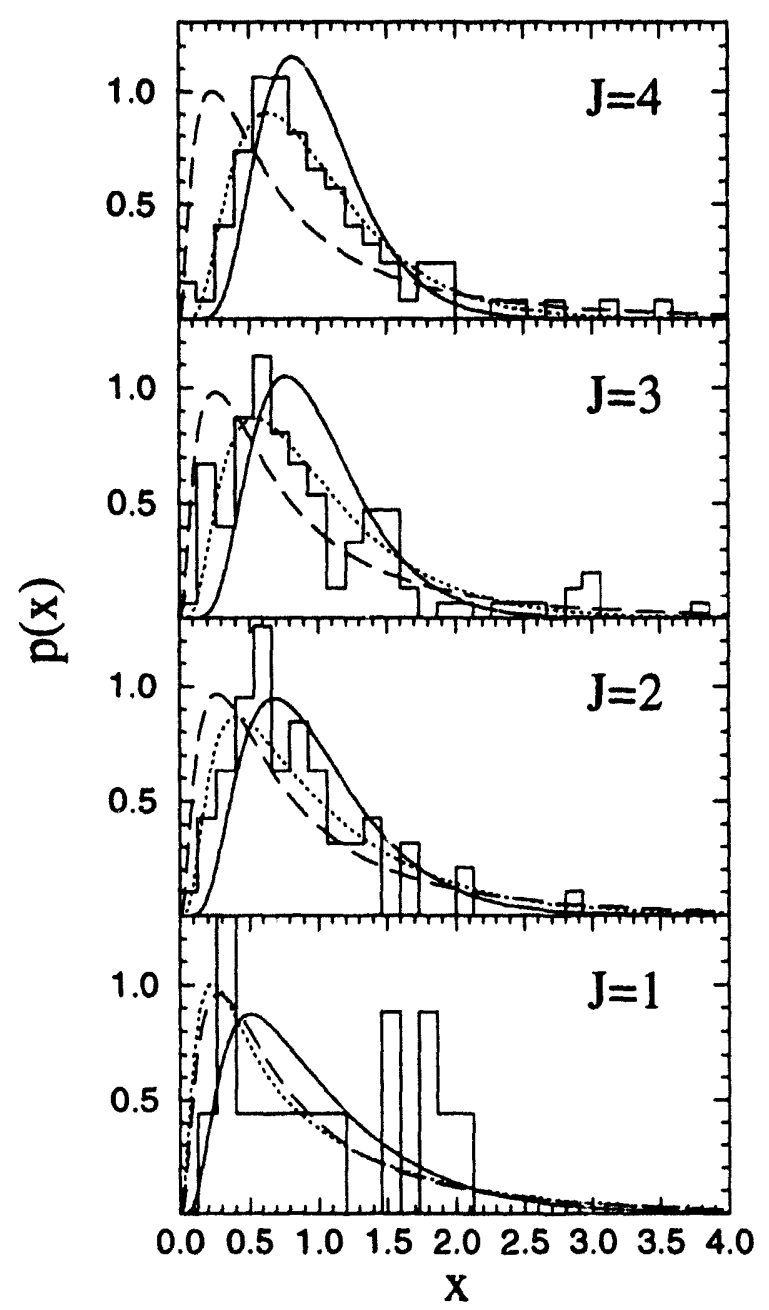

High E-Field

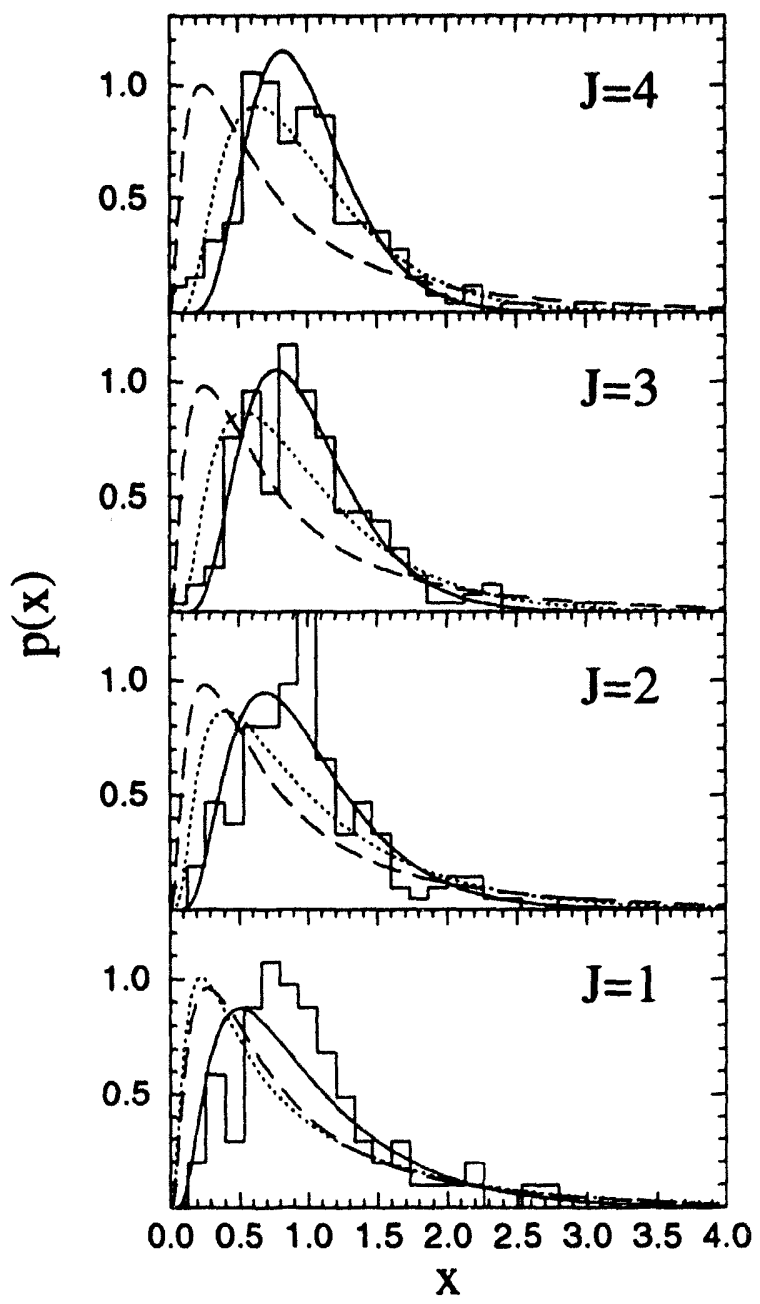

Figure 6.8: J-Resolved RM/TST probability distributions at the energy of the experiment compared to the experimental histogram for rates at low electric field (left) and at high electric field (right). The solid [case (a)], short-dashed [case (b)] and the long-dashed [case (c)] curves in both the low and high electric field panels are the corresponding RM/TST distributions presented in Fig. 6.5. As is evident the statistics for some of the histograms are rather poor, but the results are in general suggestive of what was seen for the combined distribution in Fig. 6.6. 
not enough experimental decay rates to obtain good statistics in every case, the results for $J=4$, for example, and the combined distributions exhibit a shift in the peaks from the low electric field histograms to the high electric field histograms which is mimiced by the RM/TST distributions from case (b) to case (a). (Note that, the values of $\nu_{\text {eff }}$ for these electric field cases are compared to the RM/TST values in Table 6.4.) The agreement is remarkable and suggests that $C_{s}$ symmetry is approximately conserved at low electric field, while at sufficiently high electric field the symmetry is completely broken. Nonetheless, while this markedly different behavior in the experimental distributions is mimicked by the breaking of $C_{S}$ symmetry, the possibility that the breakdown of $J$ by the Stark field could account for this behavior has not been ruled out.

\subsection{Concluding Remarks}

Application of the RM/TST theory to the distribution of state-specific unimolecular decay rates of $\mathrm{D}_{2} \mathrm{CO}$ for individual values of total angular momentum $J$ shows quite good agreement with the experimental results of Polik et al. ${ }^{21}$ In particular, if one assumes that all of the $K$ states are accessed in the experiment and that $|K|$ is strongly mixed, one obtains very good agreement between RM/TST and the experiment; the differences between the low and high field distributions may be attributable to the breaking of $C_{s}$ symmetry by the Stark field. However, this agreement may be deceptive in that previous analysis ${ }^{21,99}$ of the experimental data suggest that $|K|$ is not strongly mixed. In this case, the RM/TST results suggest that if one is to treat $|K|$ as conserved then one must allow $J$ to break down. Thus while this work does not unambiguously resolve the issue as to which rovibrational states are strongly mixed, there seems little doubt that some states are strongly mixed and that the Stark field is inducing further mixing.

The picture which seems to emerge from this analysis is that although certain dynamical quantities may be conserved locally - for example in the quasi-bound region - these quantities need not be conserved throughout the entire dynamics of the scattering event. In the present study, this has been exhibited for the quantum 
numbers associated with angular momentum. Independently, Hase and coworkers ${ }^{169}$ have also suggested models in which $K$ is conserved (adiabatic) or strongly mixed (active) in the transition state region independently of whether $K$ in the quasi-bound region is treated as conserved or strongly-mixed. 


\section{Chapter 7}

\section{Discussion}

In this work, we have developed a semiclassical transition state theory which can be used to obtain the cumulative reaction probability (CRP) and thermal rates for large systems. Use of this theory in conjunction with a statistical theory describing the dynamics of the quasi-bound reactants has led to the development of the random matrix / transition state theory (RM/TST) that describes the distributions of unimolecular decay rates. All that remains is a discussion of the future directions which this work now suggests.

\section{$7.1 \quad$ SCTST}

To date, SCTST has been applied only to a handful of systems. ${ }^{103,104,170}$ It will certainly be interesting to see how well the theory can agree with experiment for large gas-phase systems. As a prerequisite to the calculation, however, one needs to obtain the "spectroscopic constants" of the activated complex. In the initial work there was much optimism that through the use of $a b$ initio analytic higher order derivative calculations, a catalogue of such constants describing the activated complex of various molecular systems would soon be available. In fact, the original calculation on the $\mathrm{D}_{2} \mathrm{CO}$ dissociation was performed with such a set of "spectroscopic constants." 103 Unfortunately, this promise has remained largely unfulfilled, and the remaining applications to date have been performed using force constants obtained by 
finite differences on known surfaces. ${ }^{104,126}$ Given that, in principle, it should be easier to obtain only the local information of the transition state then it is to construct an entire potential energy surface, the utility of SCTST to describe thermal rates of large reactive systems certainly rests on the feasibility of $a b$ initio force constants of the activated complex, and these should become increasingly available in the near future. (This statement has an obvious proviso: the saddle point on the potential energy surface corresponding to the activated complex is not known a priori and the search for this point may involve an effort equal to that needed to obtain the points for the entire potential energy surface.)

The SCTST thermal rate expression developed in Chapter 4 has a very appealing interpretation in light of the theory of the activated complex. Its form suggests that there is a well-defined sense in which the activated complex can be described by a partition function even when the reactive degree of freedom is not entirely separable from it. The $\theta$-dependence of the pre-reactive function thus coutains the non-separable dynamics. By the use of the Weyl correspondence, we have thus suggested a quantum mechanical form of the reactive partition function whose Hamiltonian is parametrized by the generalized barrier penetration integral $\theta$.

The use of mixed-diagonalization in SCTST explored in Chapter 4 also offers a novel attempt to construct more accurate rates than those offered by the perturbative SCTST in which the anharmonicity of the perpendicular modes can be treated exactly rather than perturbatively. The possible improvement offered by this method, however, needs to be compared to the additional effort which it entails. In the form presented, the dimensionality of the exact diagonalization has only been reduced by 1 , and so the limitations on the feasibility of completely exact calculations are also limitations on this method. Here, however, one can consider treating only some of the modes by diagonalization in an obvious generalization of the formula presented. The difficulty with this procedure would lie in obtaining a systematic method by which to choose the modes to be treated perturbatively, and this has yet to be explored.

The limit of this attempt to systematically improve the semiclassical formula is evidently the correct quantum mechanical expression. Of note is the work of 
Seideman and Miller ${ }^{112}$ in which the eigenenergies of the activated complex obtained perturbatively in SCTST were shown to correspond to the exact Siegert eigenvalues ${ }^{144}$ of the system. Here the use of mixed-diagonalization permits this correspondence to persist beyond those eigenenergies in which the quantum number assignments are lost. Similarly the eigenreaction probabilities of Manthe and Miller ${ }^{171}$ are seemingly related to the transmission probabilities of the activated complex. Although not shown, the disagreement between the eigenreaction probabilities of Manthe and Miller and the perturbative SCTST is a manif,station that not all of the non-separable effects of the coupling between the reaction coordinate are included in the SCTST. The most important of these is that scattering resonances are still not described properly in the SCTST, and this drawback is not removable within the TST spirit of the method.

An ambitious direction that should be pursued is to use SCTST to develop a semiclassical theory for the reaction rates of truly large systems whose dynamics may be described by a generalized Langevin equation (GLE). In this application one takes advantage of the isomorphism between the GLE and the Hamiltonian describing a one-dimensional reactive degree of freedom bilinearly coupled to an infinite bath of uncoupled harmonic oscillators. ${ }^{172,173}$ This Hamiltonian system can certainly be cast in the form which is prerequisite for the SCTST through an expansion about the barrier associated with the activated complex. In principle, there in only one change in the SCTST formalism in including quantum effects semiclassically to the GLE system compared to the gas-phase systems: rather than a finite number of perpendicular modes of the gas-phase activated complex, in the GLE system there exists an infinite number of modes which must be averaged over to provide the thermal rate. How this averaging is performed will be the subject of future work.

\section{$7.2 \mathrm{RM} / \mathrm{TST}$}

The inclusion of dynamical symmetries in the RM/TST developed in Chapter 5 allows the treatment of the natural limits of symmetry conservation that can be satisfied by a given quantity; that, is the quantity is either conserved or completely mixed (and treated statistically). However, in many systems the dynamical behavior 
will lie somewhere in between, and so one is motivated to construct a theory which can account for this possibility. In the present work, this has been avoided in order to restrict the number of adjustable parameters of the theory to only a discretized set of possibilities depending on those rotational quantum numbers which are treated actively or statistically. Unfortunately this prevented a treatment of partial $J$ nonconservation in the $\mathrm{D}_{2} \mathrm{CO}$ dissociation and so this possibility could not be ruled out.

In order to extend the RM/TST theory to account for partial symmetry breaking, it is natural to consider an ensemble of block-diagonal matrices in which each block is a GOE labeled by the quantum numbers of the symmetry, as before, but one now includes off-diagonal coupling between these blocks. This ensemble has recently been proposed by Leitner. ${ }^{94}$ The difficulty in implementing this procedure in RM/TST is that one now needs to consider the matrix elements of the decay rate matrix $\Gamma$ between states which do not uniformly sample the same Hilbert space. One possible way to get around this difficulty is to only consider coupling between adjacent blocks of the bock-diagonal Hamiltonian - an ensemble of banded block-diagonal matrices - and to further treat the coupling perturbatively.

Conceptually the most interesting question that this work has explored is: What is the origin of the fluctuations of the state-specific decay rates? Wolynes and coworkers $^{174}$ have used a semiclassical path analysis to calculate a given rate constant and their results lead them to suggest that "fluctuations in the state-specific rates from the average depend upon the probability of the system to recross the transition state." 174 However, the RM/TST developed in this work intrinsically uses a transition state theory to describe $\Gamma$ and as such does not include recrossings; yet the distributions evidently exhibit fluctuations about the average rate. The answer does not seem to be that they result from the unique properties of a given modespecific state, as these were treated statistically. The only solution that remains is that quantization of the activated complex - i.e., the differing weights of each of the decay channels - is at the origin of the fluctuations, and this behavior can be accounted for within a semiclassical TST, but not a classical one. 


\subsection{Final Cbservations}

At the outset of this work two competing paradigms were introduced to describe energy transfer during dissociation: complete IVR in the RRKM theory verses no IVR in Slater's theory. The results of this work suggest that intermediate approaches between these extremes - in which some aspects of the dynamics is treated adiabatically and others statistically - can offer a better description of observed phenomena. In order to treat systems larger than those for which exact theories are numerically feasible, the semiclassical method developed in this work can be used to treat the non-statistical dynamics.

It may seem counterintuitive to use a statistical method in order to describe state-specific information, however only the dynamics which determine the observed state-specific behavior need be treated non-statistically. This, of course, depends on the nature of the behavior of interest; if, for example, the correlation between energy levels and their decay rates was desired then it would have been necessary to obtain the full dynamics and not just that of the activated complex. In this work only the fluctuations of decay rates were explored and for this quantity, only the local dynamics of the transition state were needed. 


\section{References}

[1] D. C. Chatfield, D. G. Truhlar, and D. W. Schwenke, J. Chem. Phys. 94, 2040 (1991).

[2] D. K. Bondi and J. N. L. Connor, J. Chem. Phys. 82, 4385 (1985).

[3] N. B. Slater, Theory of Unimolecular Reactions (Cornell University Press, Ithaca, New York, 1959).

[4] O. K. Rice and H. C. Ramsberger, J. Am. Chem. Soc. 49, 1617 (1927).

[5] L. S. Kassel, J. Phys. Chem. 32, 225, 1065 (1928).

[6] L. S. Kassel, Kinetics of Homogeneous Gas Reactions (Chemical Catalog Co., New York, 1932).

[7] R. A.Marcus and O. K. Rice, J. Phys. Coll. Chem. 55, 894 (1951).

[8] H. M. Rosenstock, M. B. Wallenstein, A. L. Wahrhaftig, and H. Eyring, Proc. Nat. Acad. Sci. U. S. 38, 667 (1952).

[9] R. A. Marcus, J. Chem. Phys. 20, 359 (1952).

[10] G. M. Wieder and R. A. Marcus, J. Chem. Phys. 37, 1835 (1962).

[11] R. A. Marcus, J. Chem. Phys. 43, 2658 (1965).

[12] W. Forst, Theory of Unimolecular Reactions (Academic, New York, 1973).

[13] T. Uzer, Phys. Rev. 199, 73 (1991); and references therein. 
[14] K. S. Pitzer, F. T. Smith, and H. Eyring, The Transition State, No. 16 in Special Publ. (Chemical Society, London, 1962), p. 53.

[15] W. H. Miller, Acc. Chem. Res. 26, 174 (1993).

[16] U. Manthe, T. Seideman, and W. H. Miller, J. Chem. Phys. 99, 0000 (1993).

[17] R. A. Marcus, J. Chem. Phys. 45, 4493, 4500 (1966); 49, 2610 (1967).

[18] R. A. Marcus, J. Chem. Phys. 49, 2610 (1968).

[19] R. E. Wyatt, J. Chem. Phys. 51, 3489 (1969).

[20] D. G. Truhlar, J. Chem. Phys. 53, 2041 (1970).

[21] W. F. Polik, D. R. Guyer, and C. B. Moore, J. Chem. Phys. 92, 3453 (1990).

[22] M. C. Gutzwiller, J. Math. Phys. 8, 1979 (1967).

[23] M. C. Gutzwiller, J. Math. Phys. 10, 1004 (1969).

[24] M. C. Gutzwiller, J. Math. Phys. 11, 1791 (1970).

[25] M. C. Gutzwiller, J. Math. Phys. 12, 343 (1971).

[26] S. T. of Bound States, Adv. Chem. Phys. 36, 1 (1977).

[27] W. H. Miller, J. Chem. Phys. 61, 4301 (1974).

[28] M. V. Berry and K. E. Mount, Rep. Prog. Phys. 35, 315 (1972).

[29] W. H. Miller, Adv. Chem. Phys. 25, 69 (1974).

[30] J. B. Delos, Adv. Chem. Phys. 65, 161 (1986).

[31] M. S. Child, Semiclassical mechanics with molecular applications (Oxford University Press, New York, 1991), p. 272 et seq.

[32] R. G. Littlejohn, J. Stat. Phys. 68, 7 (1992). 
[33] L. Brillouin, Comptes Rendus 183, 24 (1926).

[34] G. Wentzel, Zeits. f. Physik 38, 517 (1926).

[35] H. A. Kramers, Zeits. f. Physik 39, 828 (1926).

[36] H. Jeffreys, Proc. London Math. Soc. 23, 428 (1923).

[37] H. Goldstein, Classical Mechanics (Addison-Wesley, Reading, Massachusetts, 1980), p. 272 et seq.

[38] W. H. Miller, J. Chem. Phys. 61, 4301 (1974).

[39] R. E. Langer, Phys. Rev. 51, 545 (1937).

[40] M. C. Gutzwiller, Chaos in Classical and Quantum Mechanics (Springer-Verlag, New York, 1990).

[41] W. H. Miller, Faraday Discussions Chem. Soc. 62, 40 (1977).

[42] Faraday Discussions Chem. Soc. 34, 1 (1938); herein is a transcript of a general discussion "on the theoretical methods of treating activation energy and reaction velocity" among whose contributors are H. Eyring, M. G. Evans, M. Polanyi, E. Wigner, N. B. Slater, and C. N. Hinshelwood.

[43] E. P. Wigner, Faraday Discussions Chem. Soc. 34, 29 (1938).

[44] J. C. Tully, in Modern Theoretical Chemistry, edited by W. H. Miller (Plenum, New York, 1976), Vol. 2, p. 217.

[45] P. Pechukas, in Modern Theoretical Chemistry, edited by W. H. Miller (Plenum, New York, 1976), Vol. 2, p. 269.

[46] W. H. Miller, Acc. Chem. Res. 9, 306 (1976).

[47] P. Pechukas, Ann. Rev. Phys. Chem. 32, 159 (1981).

[48] D. G. Truhlar and B. C. Garrett, Ann. Rev. Phys. Chem. 35, 159 (1984). 
[49] D. G. Truhlar, A. D. Issacson, and B. C. Garret, in Theory of Chemical Reaction Dynamics, edited by M. Baer (CRC, Boca Raton, Florida, 1985), Vol. 4.

[50] P. Pechukas and F. J. Lafferty, J. Chem. Phys. 58, 1622 (1973).

[51] E. P. Wigner, J. Chem. Phys. 5, 720 (1937).

[52] J. C. Keck, Adv. Chem. Phys. 13, 85 (1967).

[53] P. Pechukas and E. Pollak, J. Chem. Phys. 71, 2062 (1979).

[54] R. A. Marcus, J. Phys. Chem. 83, 204 (1979).

[55] E. Pollak, Chem. Phys. 61, 305 (1981).

[56] F. J. Lafferty and P. Pechukas, Chem. Phys. Let. 27, 511 (1974).

[57] J. W. Tromp and W. H. Miller, J. Phys. Chem. 90, 3482 (1986).

[58] B. C. Garret and D. G. Truhlar, J. Phys. Chem. 83, 1079 (1979).

[59] B. C. Garret, A. D. Isaacson, R. T. Skodje, and D. G. Truhlar, J. Phys. Chem. 86, 2252 (1982).

[60] M. Abramowitz and I. A. Stegun, Handbook of Mathematical Functions (U.S. Govt. Printing Office, Washington, D.C., 1965).

[61] T. A. Brody et al., Rev. Mod. Phys. 53, 385 (1981).

[62] L. Wilets, Phys. Rev. Lett. 9, 430 (1962).

[63] C. E. Porter and R. G. Thomas, Phys. Rev. 104, 483 (1956).

[64] M. G. Kendall, The Advanced Theory of Statistics (Griffin, London, 1946).

[65] B. W. Silverman, Density Estimation for Statistics and Data Analysis (Chapman and Hall, London, 1986).

[66] Y. Alhassid and R. D. Levine, Chem. Phys. Let. 73, 16 (1980). 
[67] J. L. Kinsey and R. D. Levine, Chem. Phys. Let. 65, 413 (1979).

[68] R. D. Levine, Adv. Chem. Phys. 70, 53 (1987); and references therein.

[69] C. E. Porter, Statistical Theories of Spectra: Fluctuations (Academic, New York, 1965).

[70] J. D. Garrison, Ann. Phys. 30, 269 (1964).

[71] C. E. Porter, Phys. Lett. 2, 292 (1962).

[72] R. D. Levine, Adv. Chem. Phys. 47, 239 (1981); and references therein.

[73] J. Jortner, R. D. Levine, and S. A. Rice, Adv. Chem. Phys. 70, 1 (1987); and references therein.

[74] E. P. Wigner, Ann. Math. 53, 36 (1951); 62, 548 (1955); 65, 203 (1957); 67, 325 (1958).

[75] F. J. Dyson, J. Math. Phys. 3, 140, 157, 166 (1962).

[76] F. J. Dyson and M. L. Mehta, J. Math. Phys. 4, 701 (1963).

[77] E. P. Wigner, SIAM Rev. 9, 1 (1967).

[78] M. L. Mehta, Random Matrices and the Statistical Theory of Energy Levels (Academic, New York, 1967).

[79] O. Bohigas and M. J. Giannoni, Mathematical and Computational Methods in Nuclear Physics, No. 209 in Lecture Notes in Physics (Springer-Verlag, New York, 1984).

[80] R. Jost and M. Lombardi, Quantum Chaos and Statistical Nuclear Physics, edited by T. H. Seligman and H. Nishioka (Springer-Verlag, New York, 1986), Vol. 92 , p. 72 , and references therein.

[81] J. P. Pique, Y. Chen, R. W. Field, and J. L. Kinsey, Phys. Rev. Lett. 58, 475 (1987); and references therein. 
[82] K. K. Lehmann and S. L. Coy, Ber. Bunsenges Phys. Chem. 92, 306 (1988).

[83] G. Persch et al., Ber. Bunsenges Phys. Chem. 92, 306 (1988).

[84] T. Zimmermann et al., Phys. Rev. Lett. 61, 3 (1988).

[85] M. J. Davis and E. J. Heller, J. Phys. Chem. 86, 2118 (1982).

[86] S. A. Rice and R. Kosloff, J. Phys. Chem. 86, 2153 (1982).

[87] W. P. Reinhardt, J. Phys. Chem. 86, 2158 (1982).

[88] B. J. Berne, N. D. Leon, and R. O. Rosenberg, J. Phys. Chem. 86, 2166 (1982).

[89] P. Pechukas, J. Phys. Chem. 86, 2239 (1982).

[90] M. V. Berry, Quantum Measurement and Chaos, edited by E. R. Pike and S. Sarkar (Plenum, New York, 1986), Vol. 161, p. 81, and other references in this volume.

[91] F. J. Dyson, J. Math. Phys. 3, 1199 (1962).

[92] F. J. Dyson, J. Math. Phys. 13, 90 (1972).

[93] M. Feingold, D. M. Leitner, and M. Wilkinson, Phys. Rev. Lett. 66, 986 (1991).

[94] D. M. Leitner, Phys. Rev. E 48, 0000 (1993).

[95] M. L. Goldberger and K. M. Watson, Collision Theory (John Wiley \&. Sons, Inc., New York, 1964).

[96] D. R. Guyer, W. F. Polik, and C. B. Moore, J. Chem. Phys. 84, 6519 (1986).

[97] T. Zimmermann, H. Köppel, and L. S. Cederbaum, J. Chem. Phys. 91, 3934 (1989).

[98] W. F. Polik, C. B. Moore, and W. H. Miller, J. Chem. Phys. 89, 3584 (1988).

[99] W. F. Polik, D. R. Guyer, W. H. Miller, and C. B. Moore, J. Chem. Phys. 92, 3471 (1990). 
[100] W. H. Miller, R. Hernandez, C. B. Moore, and W. F. Polik, J. Chem. Phys. 93, 5657 (1990).

[101] W. H. Miller, J. Chem. Phys. 63, 1166 (1975).

[102] S. Chapman, B. C. Garret, and W. H. Miller, J. Chem. Phys. 63, 2710 (1975).

[103] W. H. Miller, R. Hernandez, N. C. Handy, D. Jayatilaka, and A. Willetts, Chem. Phys. Let. 172, 62 (1990).

[104] M. J. Cohen, N. C. Handy, R. Hernandez, and W. H. Miller, Chem. Phys. Let. 192, 407 (1992).

[105] W. H. Green, A. Willetts, D. Jayatilaka, and N. C. Handy, Chem. Phys. Let. 169, 127 (1990).

[106] W. H. Green, A. Willetts, R. D. Amos, D. Jayatilaka, and N. C. Handy, J. Chem. Phys. 93, 4965 (1990).

[107] D. Papousek and M. R. Aliev, Molecular Vibrational-Rotational Spectra (Elsevier, Amsterdam, 1982).

[108] E. L. Sibert, J. Chem. Phys. 88, 4378 (1988).

[109] E. L. Sibert, J. Chem. Phys. 90, 2672 (1989).

[110] L. D. Landau and E. M. Lifshitz, Quantum Mechanics (Pergamon Press, Oxford, 1965).

[111] E. C. Kemble, The Fundamental Principles of Quantum Mechanics (Dover, New York, 1958).

[112] T. Seideman and W. H. Miller, J. Chem. Phys. 95, 1768 (1991).

[113] R. Hernandez, W. H. Miller, C. B. Moore, and W. F. Polik, J. Chem. Phys. 99, 950 (1993).

[114] R. P. Bell, The tunnel effect in chemistry (Chapman and Hall, New York, 1980). 
[115] E. M. Mortesen and K. S. Pitzer, The Transition State, No. 16 in Special Publ. (Chemical Society, London, 1962), p. 57.

[116] R. A. Marcus, J. Chem. Phys. 45, 4493, 4500 (1966); 49, 2610 (1967).

[117] E. A. McCullough and R. E. Wyatt, J. Chem. Phys. 54, 3578 (1971).

[118] D. G. Truhlar and R. E. Wyatt, J. Chem. Phys. 56, 2232 (1972).

[119] T. F. George and W. H. Miller, J. Chem. Phys. 57, 2458 (1972).

[120] E. L. Sibert, J. Chem. Phys. 88, 4378 (1988); 90, 2672 (1989).

[121] M. Wolfsberg, J. Chem. Phys. 50, 1484 (1969).

[122] D. G. Truhlar and A. D. Isaacson, J. Chem. Phys. 94, 357 (1991).

[123] E. L. Sibert, Comput. Phys. Commun. 51, 149 (1988).

[124] L. E. Fried and G. S. Ezra, Comput. Phys. Commun. 51, 103 (1988).

[125] E. Fermi, Z. Phys. 71, 250 (1931).

[126] M. J. Cohen, A. Willetts, and N. C. Handy, J. Chem. Phys. 99, 5885 (1993).

[127] A. J. C. Varandas, F. B. Brown, C. A. Mead, D. G. Truhlar, and N. C. Blais, J. Chem. Phys. 86, 6258 (1987).

[128] C. Eckart, Phys. Rev. 35, 1303 (1930).

[129] T. Seideman and W. H. Miller, J. Chem. Phys. 96, 4412 (1992).

[130] T. Seideman and W. H. Miller, J. Chem. Phys. 97, 2499 (1992).

[131] S. K. Gray, W. H. Miller, Y. Yamaguchi, and H. F. S. III, J. Am. Chem. Soc. 103, 1900 (1981).

[132] W. H. Miller, N. C. Handy, and J. E. Adams, J. Chem. Phys. 72, 99 (1980).

[133] W. H. Miller, J. Am. Chem. Soc. 101, 6810 (1979). 
[134] E. T. Whittaker and G. N. Watson, A Course of Modern Analysis (Cambridge University Press, Cambridge, 1902).

[135] J. R. Cary, Phys. Rep. 79, 129 (1981).

[136] I. Shavitt and L. T. Redmon, J. Chem. Phys. 73, 5711 (1980).

[137] N. Fröman and P. O. Fröman, JWKB Approximation (North-Holland, Amsterdam, 1965).

[138] H. Lenstra, (private communication).

[139] H. S. Johnston, Gas Phase Reaction Rate Theory (Ronald Press, New York, 1966).

[140] D. A. McQuarrie, Statistical Mechanics (Harper and Row, New York, 1973).

[141] D. W. Schwenke, (private communication).

[142] C. B. Moore and J. C. Weisshaar, Ann. Rev. Phys. Chem. 34, 2525 (1983).

[143] R. C. Herman and W. H. Shaffer, J. Chem. Phys. 16, 453 (1948).

[144] A. J. F. Siegert, Phys. Rev. 56, 750 (1939).

[145] R. A. Bain, J. N. Bondsley, B. R. Junker, and C. V. Sukumar, J. Phys. B 7, 2189 (1974).

[146] A. D. Issacson, C. W. McCurdy, and W. H. Miller, Chem. Phys. 34, 311 (1978).

[147] W. L. Hase, in Modern Theoretical Chemistry, edited by W. H. Miller (Plenum, New York, 1976), Vol. 2, p. 121.

[148] H. Feshbach, Annu. Rev. Nucl. Sci. 5, 357 (1958); 8, 49 (1958); Ann. Phys. 19, 287 (1962).

[149] D. F. Heller, M. L. Elert, and W. M. Gelbart, J. Chem. Phys. 69, 4061 (1978). 
[150] N. Ullah, in International Nuclear Physics Conference, edited by R. L. Becker (Academic, New York, 1966).

[151] W. H. Miller, J. Chem. Phys. 92, 4261 (1988).

[152] D. h. Lu and W. L. Hase, J. Chem. Phys. 90, 1557 (1989).

[153] I. S. Gradshtein and I. M. Ryzhik, Tables of Integrals, Seires, and Products (Academic Press, California, 1965).

[154] W. H. Miller, Adv. Chem. Phys. 30, 77 (1975).

[155] J. J. Sakurai, Modern Quantum Mechanics (Addison-Wesley, New York, 1985).

[156] T. Carrington, L. M. Hubbard, H. F. Schaefer, and W. H. Miller, J. Chem. Phys. 80, 4347 (1984).

[157] P. R. Bunker, in Vibrational Spectra and Structure, edited by J. R. Durig (Plenum, New York, 1976), Vol. 3, pp. 1-126.

[158] M. Quack, Mol. Phys. 34, 477 (1977).

[159] W. H. Miller, J. Am. Chem. Soc. 105, 216 (1983).

[160] W. Schneider and W. Thiel, Chem. Phys. Let. 157, 367 (1989).

[161] C. Møller and M. S. Plesset, J. Chem. Phys. 46, 618 (1934).

[162] A. Willetts and N. C. Handy, (private communication).

[163] W. H. Miller, J. Phys. Chem. 87, 2731 (1983); note that in this reference the out-of-plane mode is labeled as $\nu_{5}$.

[164] J. L. Duncan and P. D. Mallinson, Chem. Phys. Let. 23, 597 (1973).

[165] D. E. Reisner, R. W. Field, J. L. Kinsey, and H. L. Dai, J. Chem. Phys. 80, 5968 (1984).

[166] G. E. Scuseria and H. F. Schaefer, J. Chem. Phys. 90, 3629 (1989). 
REFERENCES

147

[167] M. Dupuis, W. A. Lester, B. H. Lengsfield, and B. Liu, J. Chem. Phys. 79, 6167 (1983).

[168] M. J. Frisch, J. S. Binkley, and H. F. Schaefer, J. Chem. Phys. 81, 1882 (1984).

[169] L. Zhu, W. Chen, W. L. Hase, and E. W. Kaiser, J. Phys. Chem. 97, 311 (1993).

[170] M. J. Cohen, Ph.D. thesis, University of Cambridge, Cambridge, UK, 1993.

[171] U. Manthe and W. H. Miller, J. Chem. Phys. 99, 3411 (1993).

[172] R. Zwanzig, J. Stat. Phys. 9, 215 (1973).

[173] A. O. Caldeira and A. J. Leggett, Phys. Rev. Lett. 46, 211 (1981).

[174] A. C. Gentile, S. A. Schofield, and P. G. Wolynes, J. Chem. Phys. 98, 7898 (1993). 

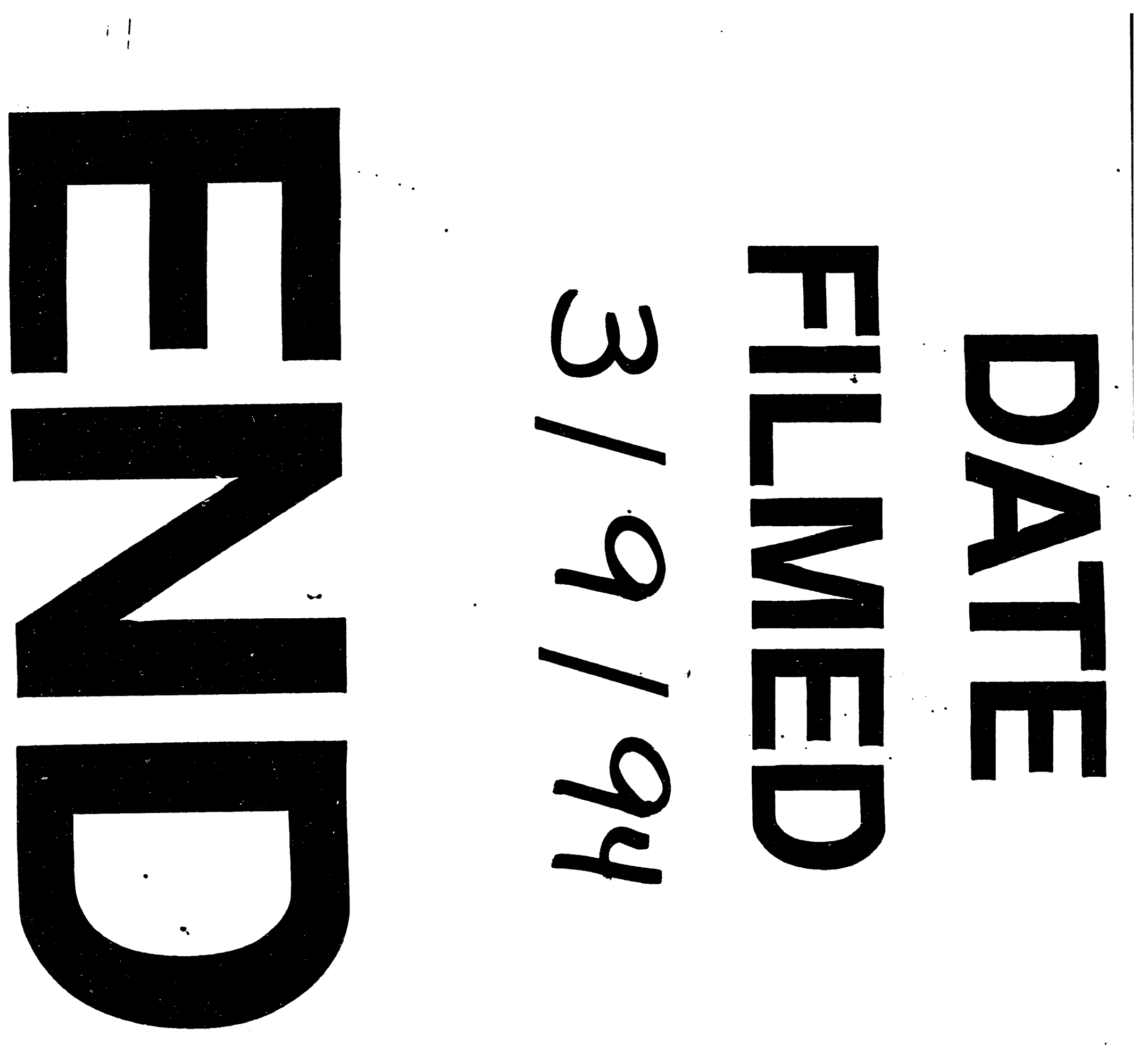


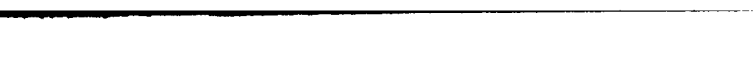

A

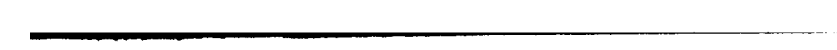
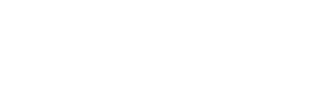$$
\text { (1) }
$$

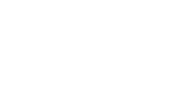
. $-$

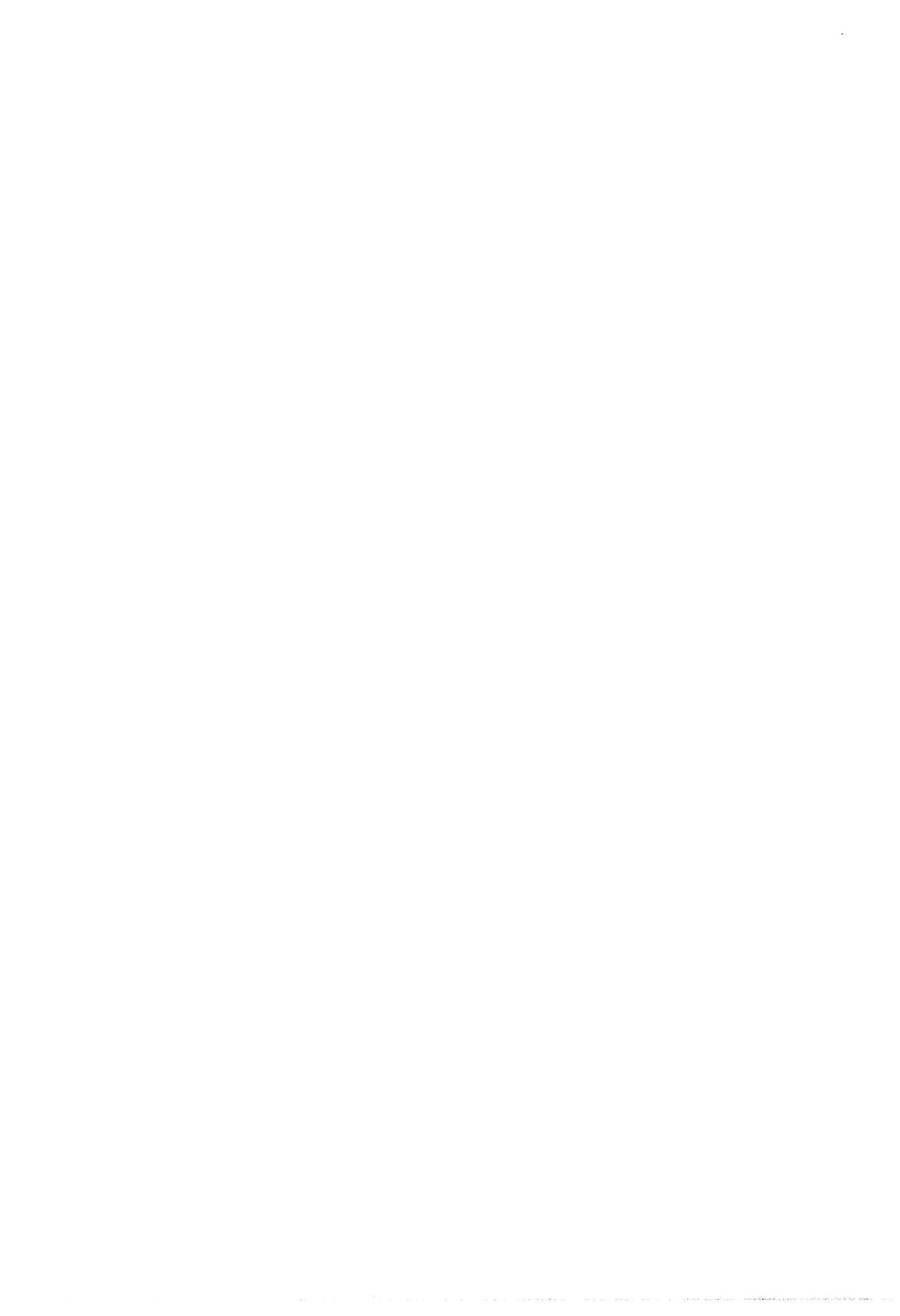

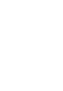

